\title{
Sketch-based modeling
}

Permission to make digital or hard copies of part or all of this work for personal or classroom use is granted without fee provided that copies are not made or distributed for profit or commercial advantage and that copies bear this notice and the full citation on the first page. Copyrights for third-party components of this work must be honored. For all other uses, contact the Owner/Author. Copyright is held by the owner/author(s).

SA '16 Courses, December 05-08, 2016, Macao

ACM 978-1-4503-4538-5/16/12.

http://dx.doi.org/10.1145/2988458.2988504

\section{Frederic Cordier}

Karan Singh

Yotam Gingold

Marie-Paule Cani 


\section{Introduction}

- Method of creating 3D models

- The user draws a 2D shape which is converted to 3D automatically
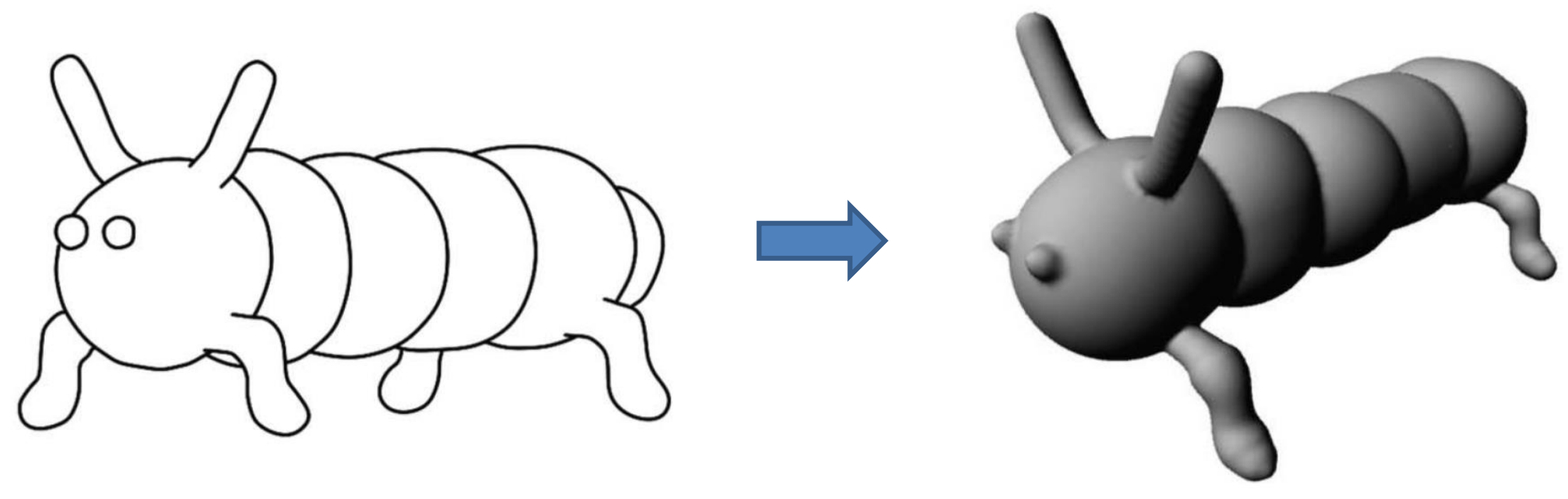


\section{Introduction}

- Existing 3D modeling programs have a high learning curve

- The purpose of sketch based modeling: making 3D modeling as simple as making a drawing

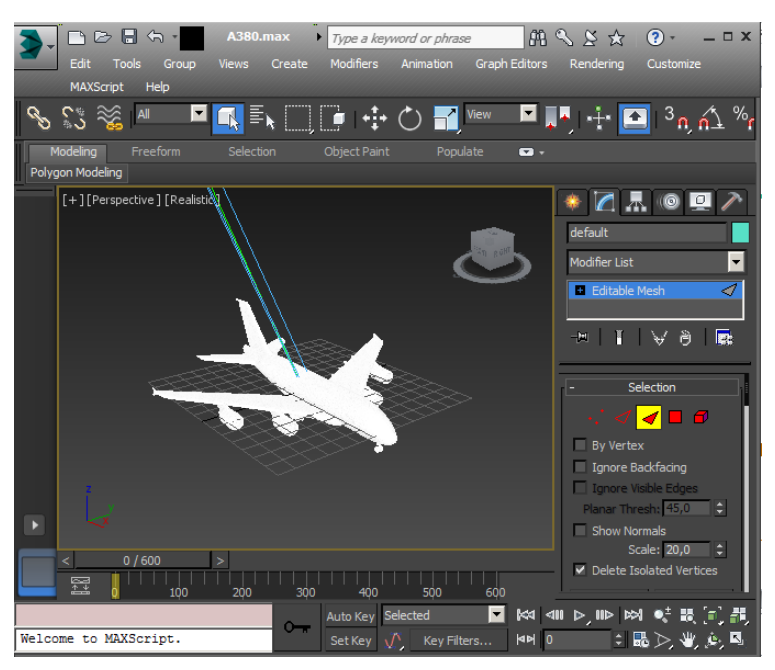




\section{Introduction}

The speakers:

- Karan Singh

Professor

University of Toronto, Canada

- Yotam Gingold

Assistant Professor

George Mason University, USA

- Marie-Paule Cani

Professor

Grenoble University (Grenoble Institute of Technology \& Inria), France

- Frederic Cordier

Associate Professor

University of Haute-Alsace and University of Strasbourg, France 


\section{Introduction}

Course topics:

- Sketch stroke acquisition and processing

- Multi-view sketch-based modeling of 3D curves and surfaces

- Sketch-based modeling using prior knowledge

- Single-view sketch-based modeling of 3D curves and surfaces

- Sketch-based editing 


\section{Sketch stroke acquisition \& processing}

Karan Singh 


\section{Issues in digital sketching}

- Stroke filtering

- Stroke processing

- Stroke dynamics

- Stroke appearance

- Stroke-based UI Control fairing, curve-fitting.

segmentation, recognition, regularization. pressure, tilt, speed, temporal order. NPR, stylization, perception. widgets, crossing, gestures. 


\section{Stroke filtering: noise \& error sources}

- User error

- Intent (wants a square but draws a rectangle).

- Execution (unsteady hand).

- Ergonomic (awkard drawing posture).

- Device error

- Input (tablets better than mice or trackpads).

- Resolution (projected better than surface capacitance).

- Signal Noise. 


\section{What are desirable strokes?}

Smoothness: "tangent and perhaps curvature continuous curves"

[Farin et al. 87]. 


\section{Simple smoothing approaches}

- Laplacian. (neighbour averaging).

- Bi-Laplacian.

- $\quad$ LSQ spline fitting. 


\section{Simple smoothing: Laplacian}

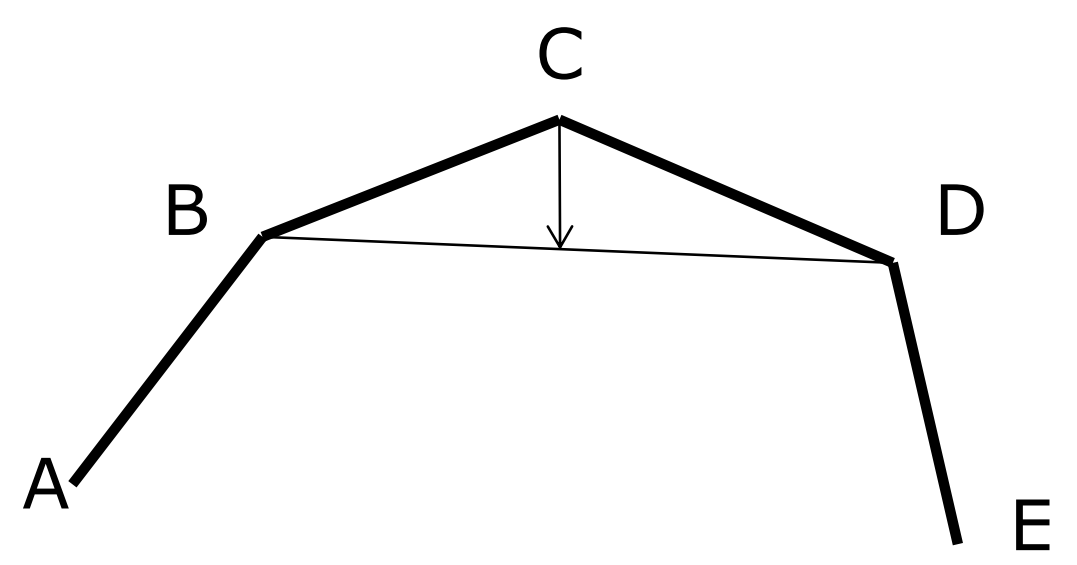

$$
\begin{aligned}
\operatorname{lap}(\mathrm{C}) & =(\mathrm{B}+\mathrm{D}) / 2-\mathrm{C} \\
\mathrm{C}^{\prime} & =\mathrm{C}+\mathrm{d}^{*} \operatorname{lap}(\mathrm{C}) \quad 0<\mathrm{d}<1
\end{aligned}
$$

Best to run many iterations with

A small $d$, for eg. 5 iterations $d=0.2$. 


\section{Simple smoothing: Bi-Laplacian}

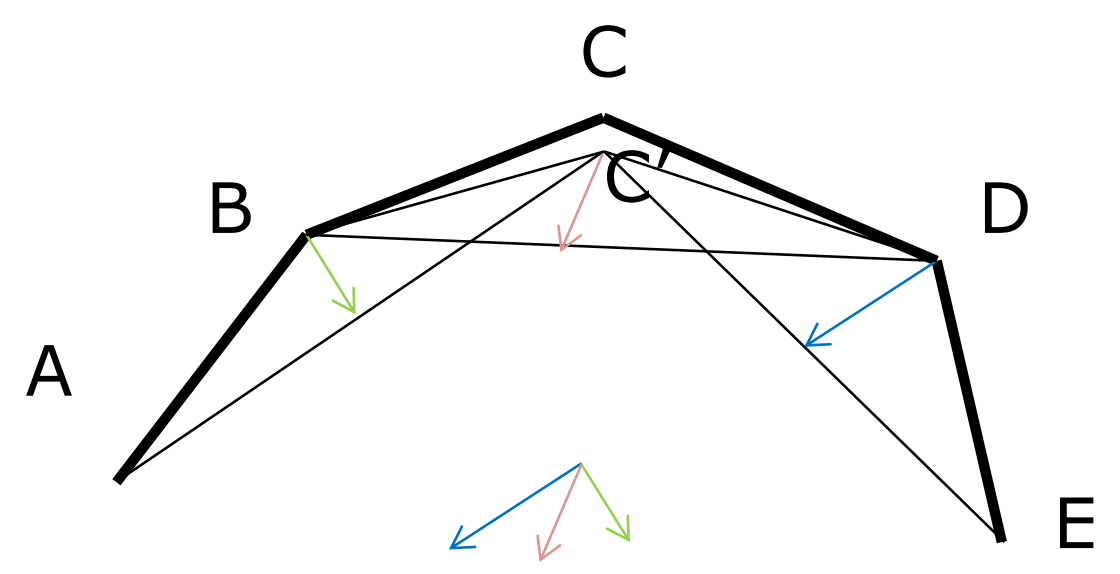

Find a $C^{\prime}$ such that:

$\operatorname{lap}\left(\mathrm{C}^{\prime}\right)=(\operatorname{lap}(\mathrm{B})+\operatorname{lap}(\mathrm{D}) \quad) / 2$

$(B+D) / 2-C^{\prime}=\left(\left(\left(A+C^{\prime}\right) / 2-B\right)+\left(\left(E+C^{\prime}\right) / 2-D\right)\right) / 2$

$C^{\prime}=2 / 3(B+D-A / 4-E / 4)$

bi-lap $(\mathrm{C})=\mathrm{C}^{\prime}-\mathrm{C}$ 


\section{Simple smoothing: LSQ fitting}

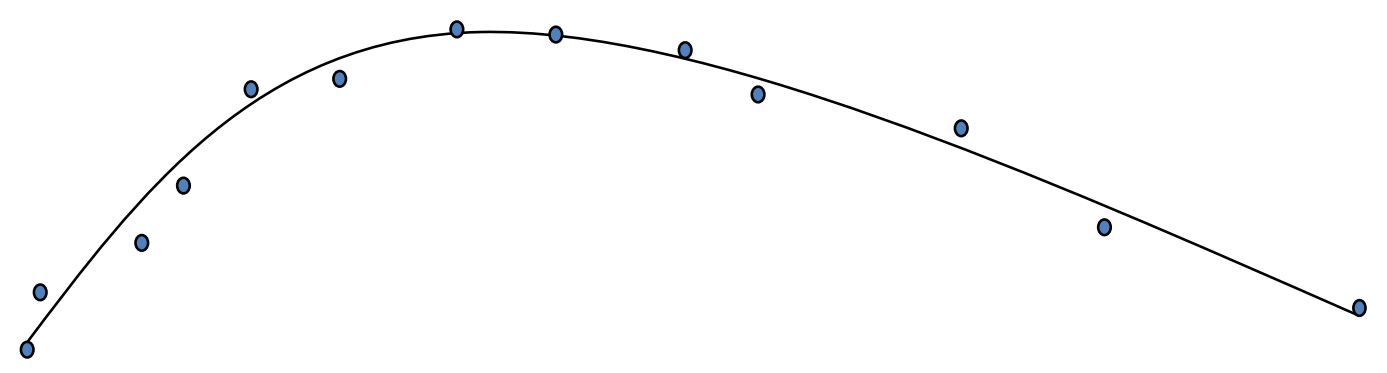

$f(t)=(x, y)$ from points $\left(x_{i}, y_{i}\right)$ 


\section{Simple smoothing: LSQ fitting}

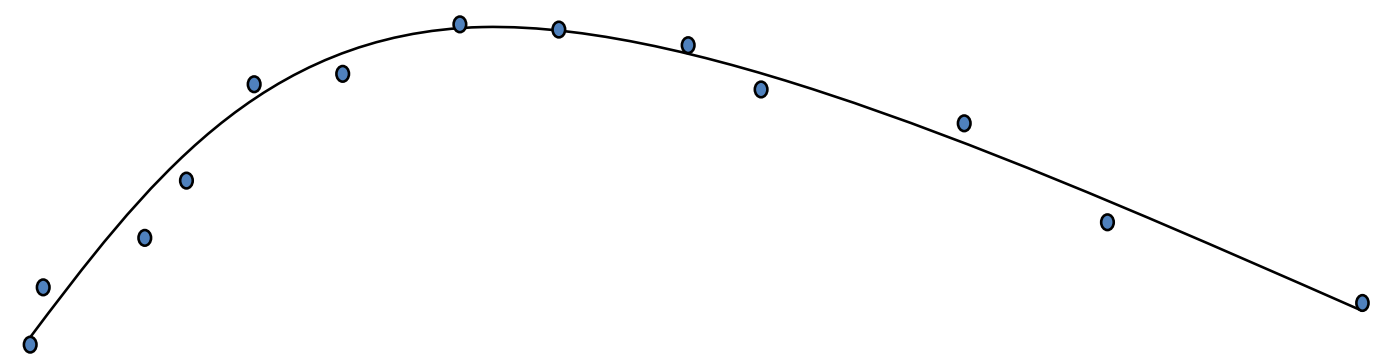

LSQ solves for $f$ to minimize error $\sum_{i}\left|f\left(t_{i}\right)-\left(x_{i}, y_{i}\right)\right|^{2}$

Approach: $\quad$ guess $t_{i}$;

LSQ solve for $f$;

refine $t_{i}$ for current $f$;

iterate... 


\section{What are desirable strokes?}

- Fairness: "curvature continuous curves with a small number of segments of almost piecewise linear curvature" [Farin et al. 87].

- Lines, circles and clothoids are the simplest primitives in curvature space.

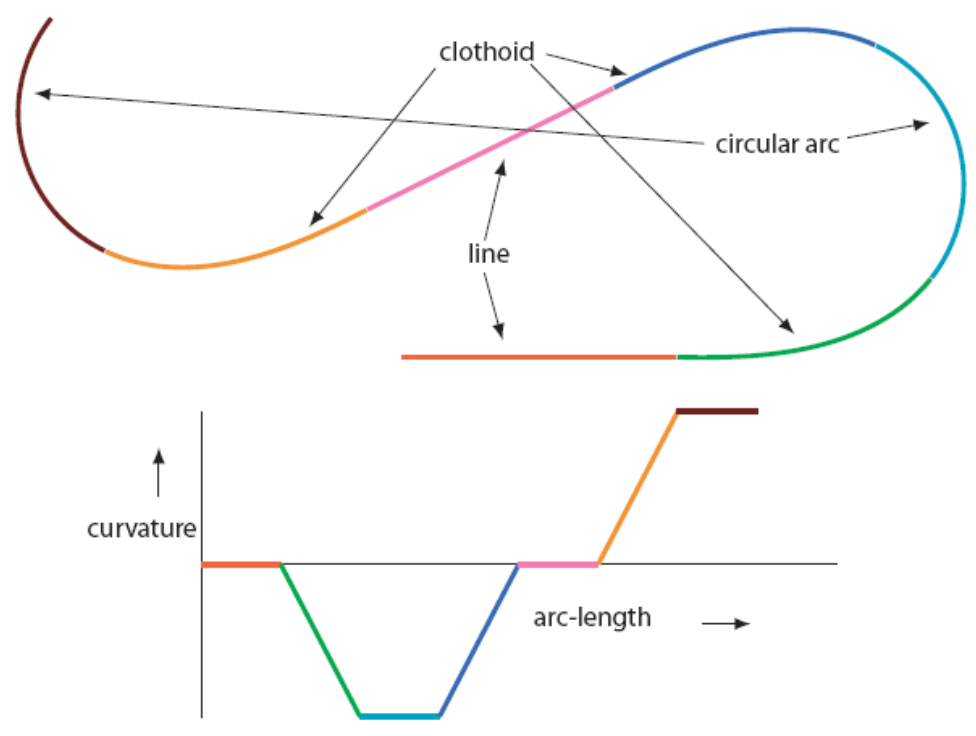




\section{Comparative approaches to fairing}

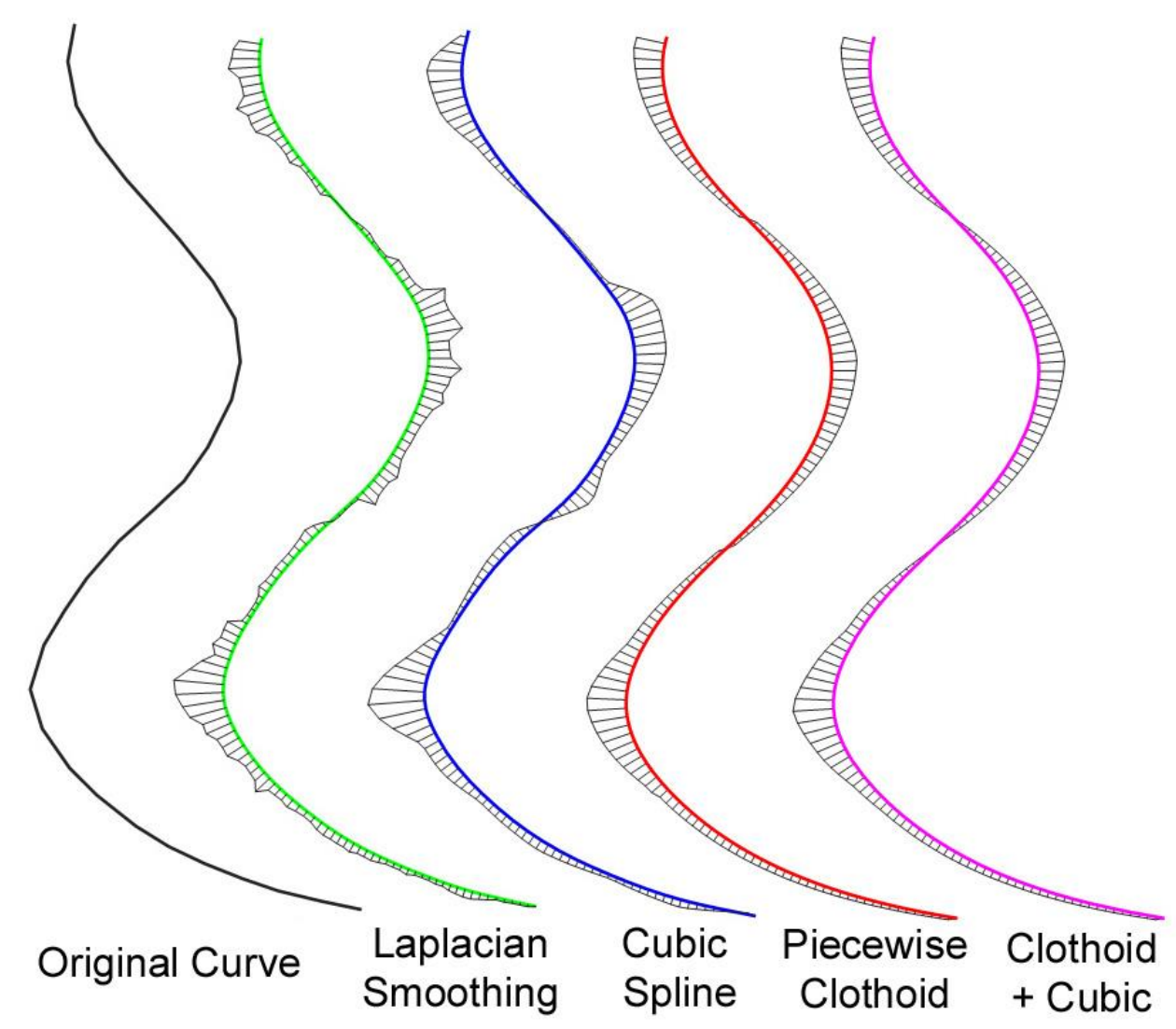

[McCrae \& Singh, Sketching Piecewise Clothoid Curves, SBIM 2008] source code: http://www.dgp.toronto.edu/ mccrae/clothoid/ 


\section{Desirable strokes}

- Neatness: "a combination of fairness and fine detail as intended by the user".

- Requires either implicit knowledge of user-intent, or an explicit neatening directive by the user. 


\section{Stroke neatening: French curves}

Physical tools, used to model curves.

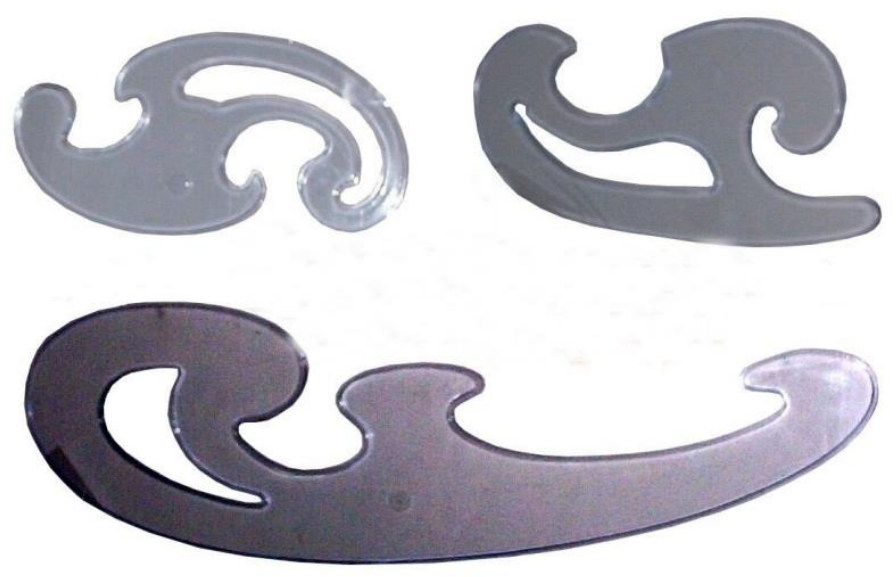

French curves +

smooth shape priors, specify a style/standard

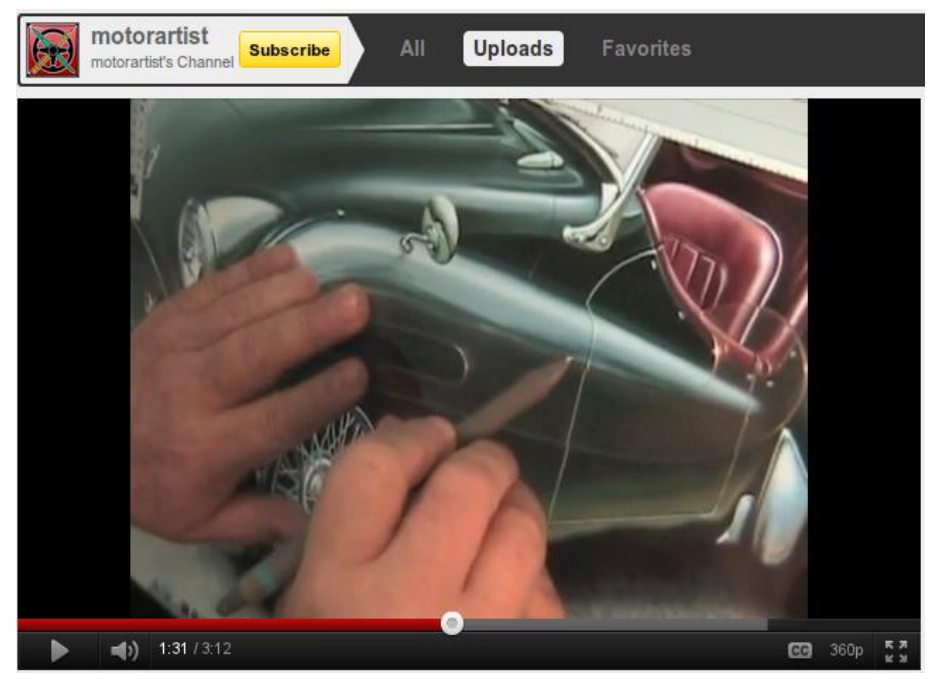

sketch interface

fluid free-form 


\section{Stroke neatening: French curves}

input polyline
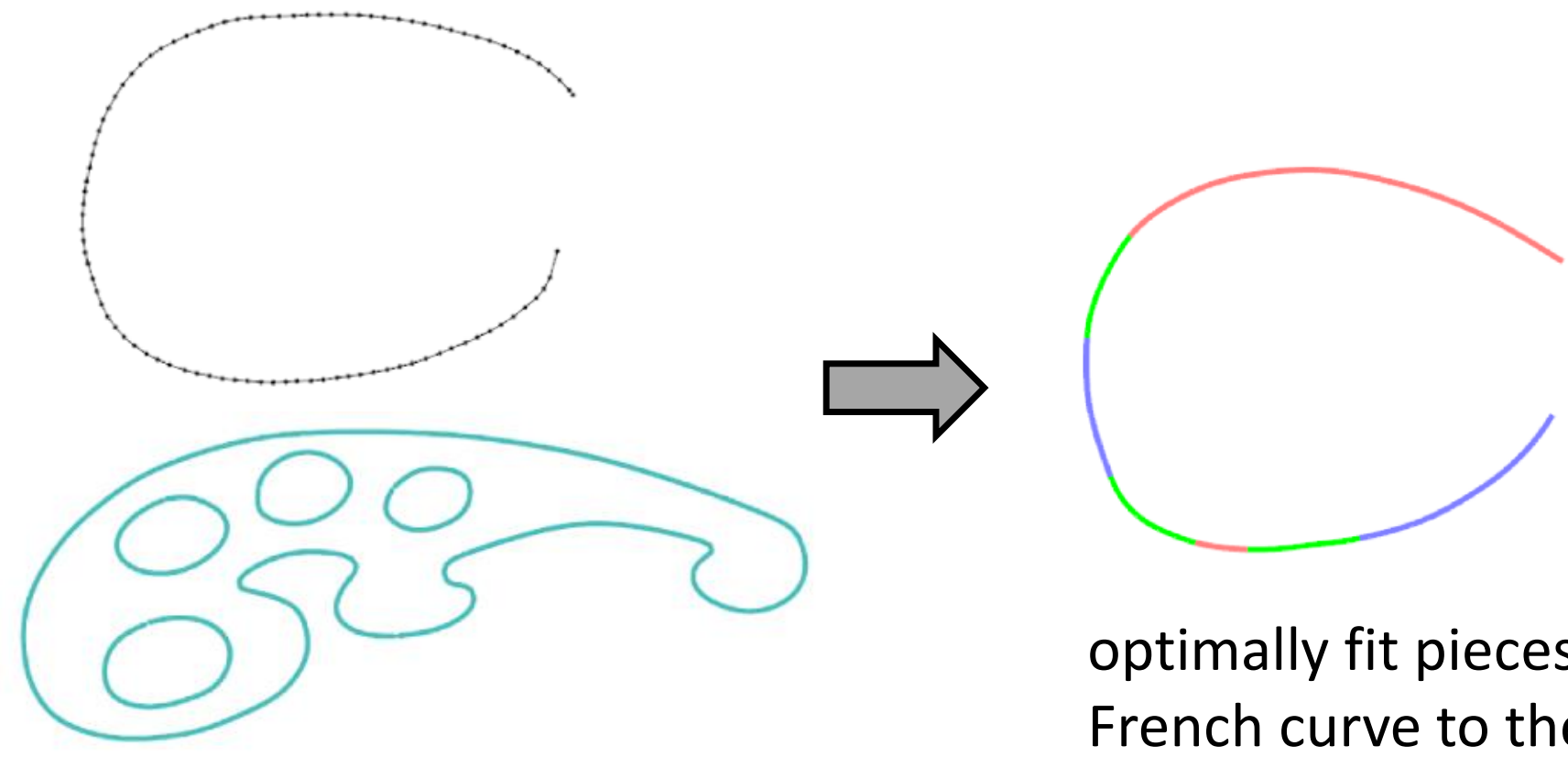

French curve

optimally fit pieces of the French curve to the input

[McCrae \& Singh, Neatening sketched strokes using piecewise French Curves, SBIM 2011] 


\section{Stroke neatening: French curves}

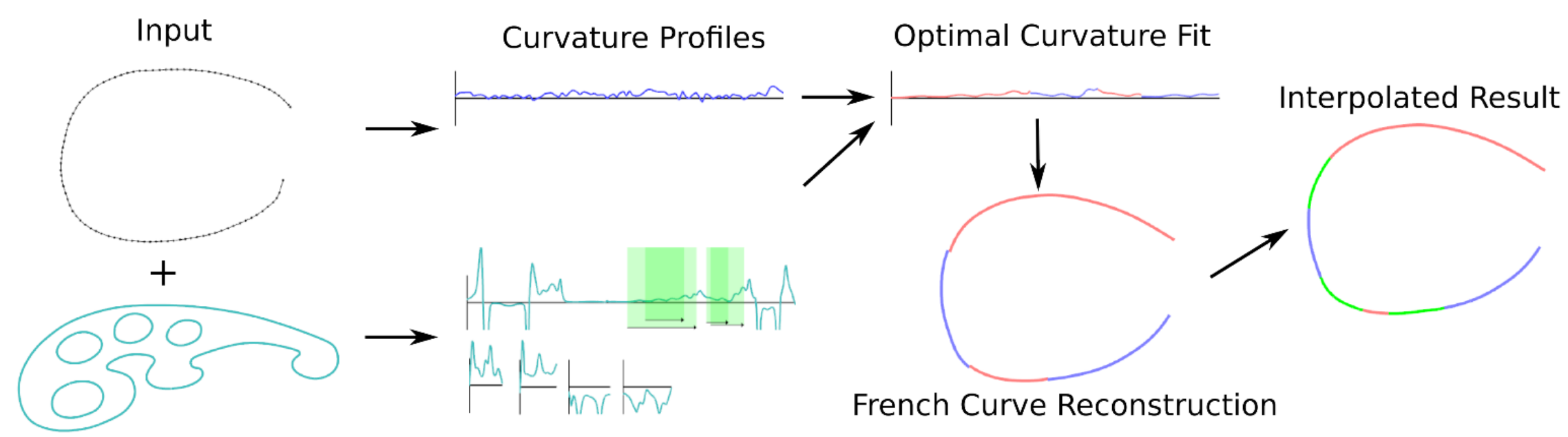




\section{Stroke neatening \& dynamics: elasticurves}
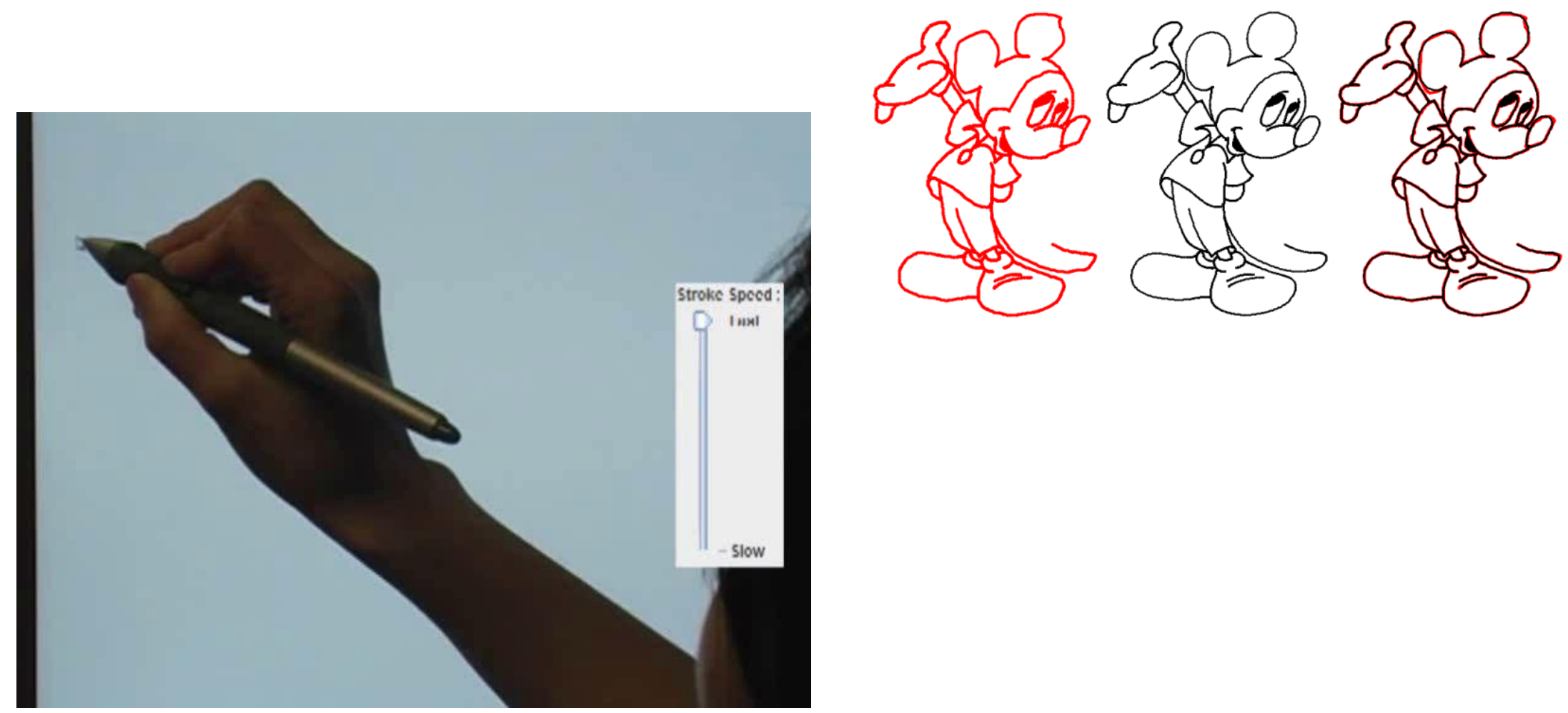

[Thiel, Singh, Balakrishnan Elasticurves: Exploiting Stroke Dynamics and Inertia for the Real-time Neatening of Sketched 2D Curves, UIST 2011] java applet: http://www.dgp.toronto.edu/ ythiel/Elasticurves/ 


\section{Elasticurve}

Input $\mathrm{q}_{\mathrm{i}}^{\prime} \mathrm{s}$ sampled at a time interval of $d t$

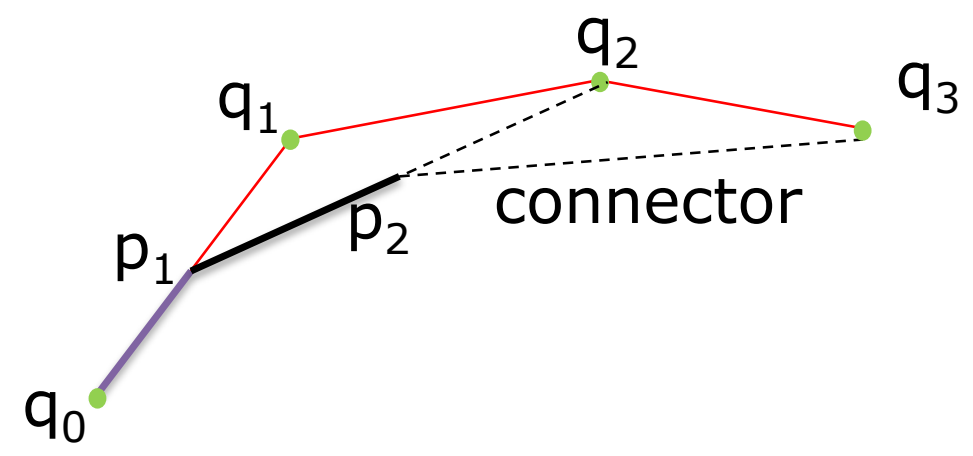

responsiveness $=$ connector arc-length fraction extending an elasticurve . 


\section{Elasticurve Properties}

- Explicit and real-time: neatness is directly correlated to drawing speed and responsiveness.

- Analytic: resilience to $d t$ sampling variation.

- Precise: embodies desirable shapes as connectors. 


\section{Elasticurve evaluation \& curve quality}

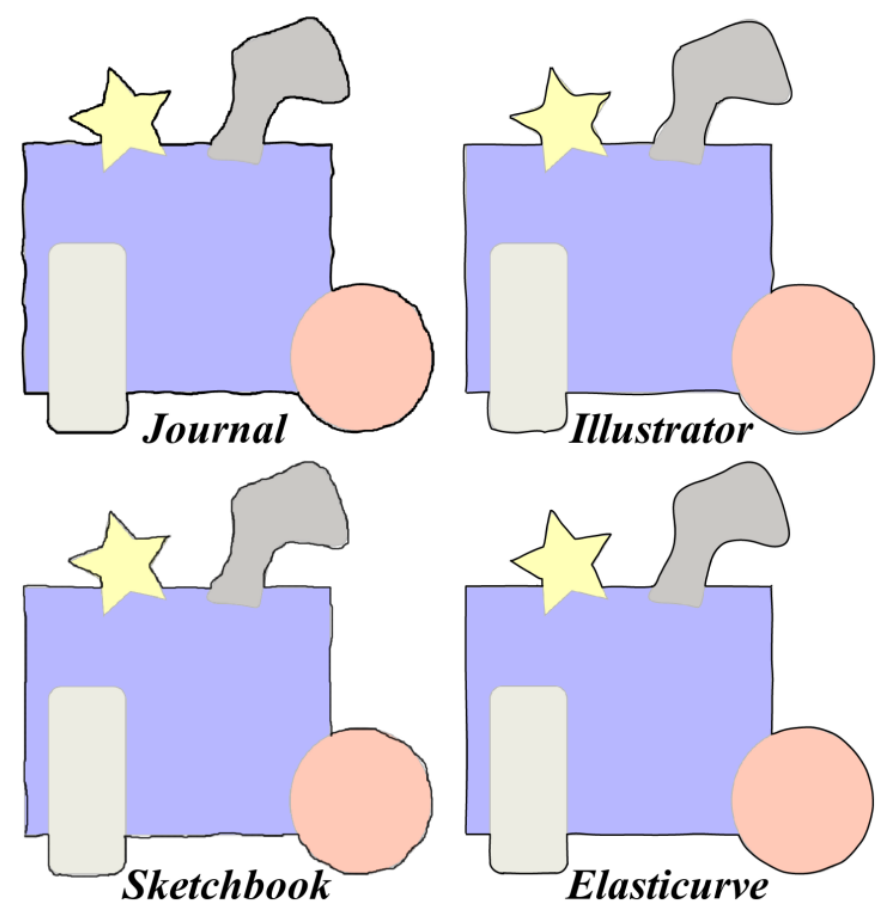

Intermediate user, trackpad, visual best of 7 attempts.

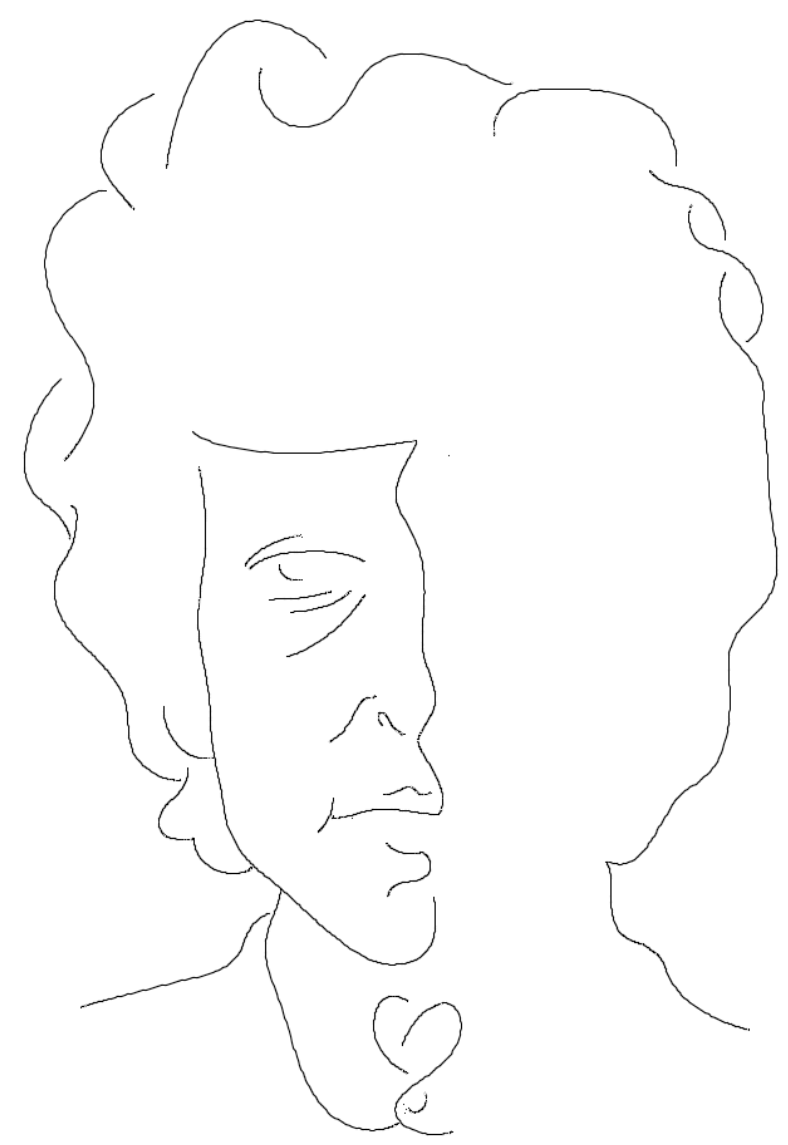




\section{Stroke Processing}

- Filtering, neatening, beautification can also be considered as stroke processing.

- Segmentation, classification, recognition.

- Regularization.

- Abstraction. 


\section{Stroke segmentation: finding corners}

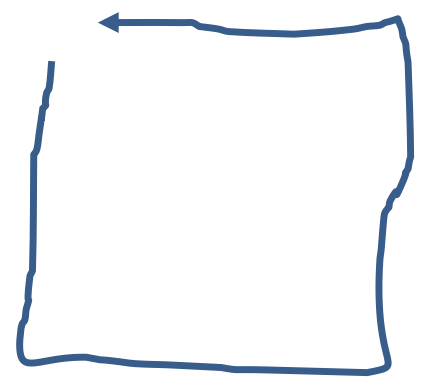

Corner detection features:

direction discontinuity speed minima curvature maxima

[T. Sezgin et al., Sketch Based Interfaces: Early Processing for Sketch Understanding, Workshop on Perceptive User Interfaces, 2001.] 


\section{Stroke classification: pentamenti}
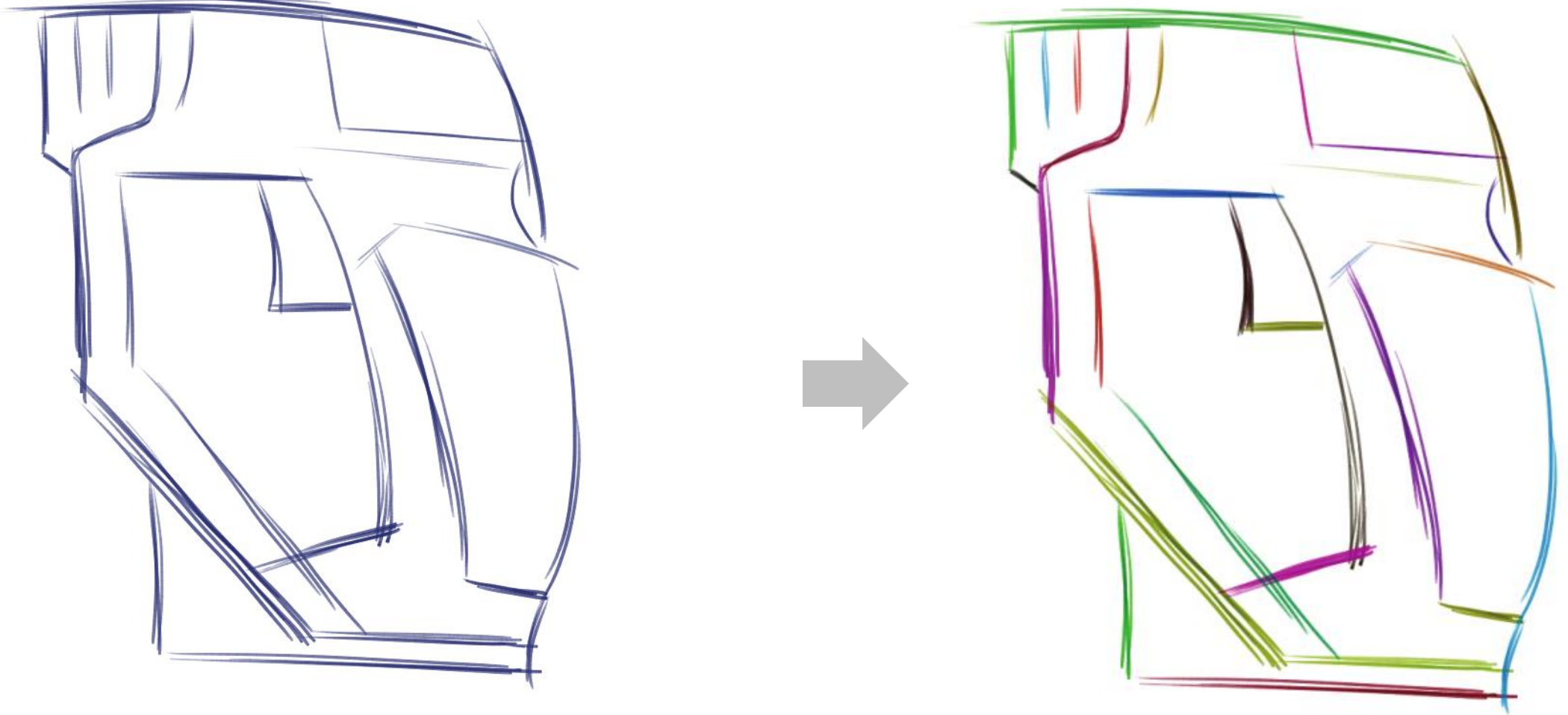

[G. Orbay \& L. Kara., Beautification of Design Sketches Using Trainable Stroke Clustering and Curve Fitting. IEEE Transactions on Visualization and Computer Graphics 17, 5 (May 2011).] 


\section{Geometric Stroke Features}
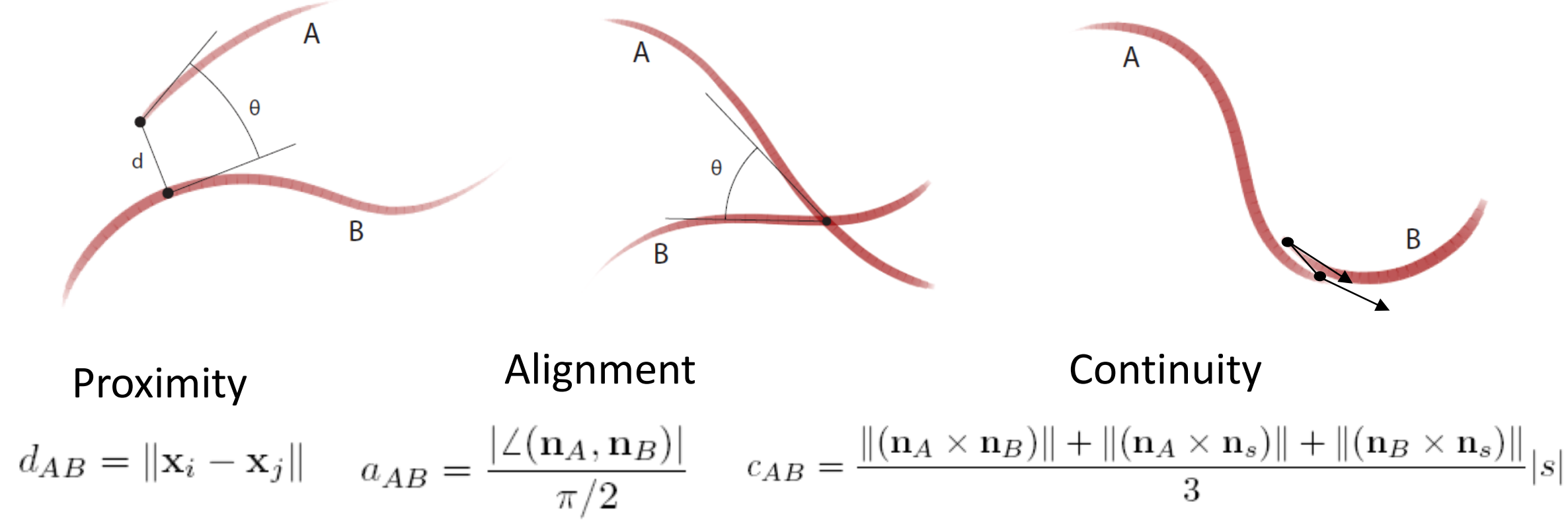

- Pairwise features

- Stroke proximity

- Local learning 


\section{Group Strokes by Affinity}

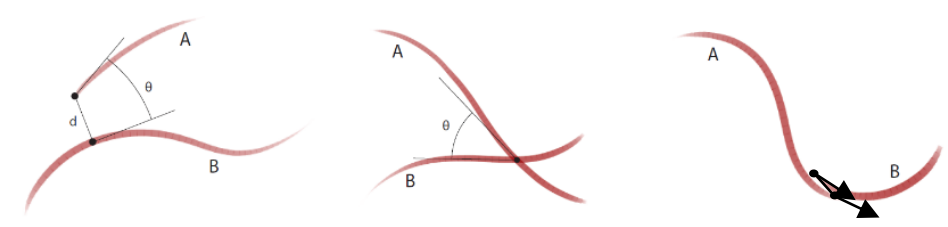

Affinity = Proximity + Alignment + Continuity

learning approaches with or without examples:

neural network

spectral clustering

greedy grouping (single-link clustering) 


\section{Stroke grouping and regularization}

Gestalt Principle

"The whole is greater than the sum of its parts" 


\section{Gestalt grouping and regularization}

- Similarity

- Symmetry

- Continuation

- Closure

- Proximity

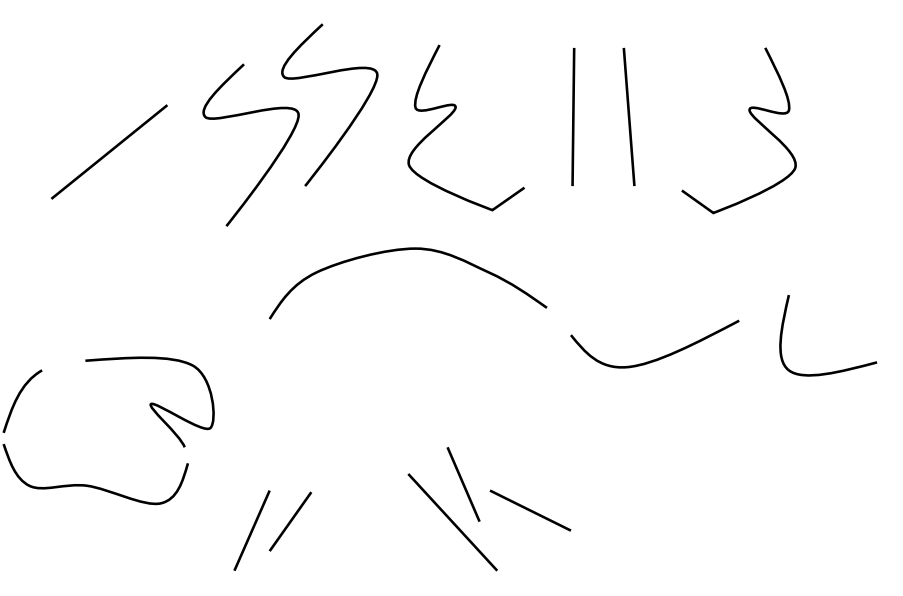

Regularization makes strokes that are nearly isometric, parallel, symmetric, perpendicular etc. precisely so! 


\section{Stroke recognition}

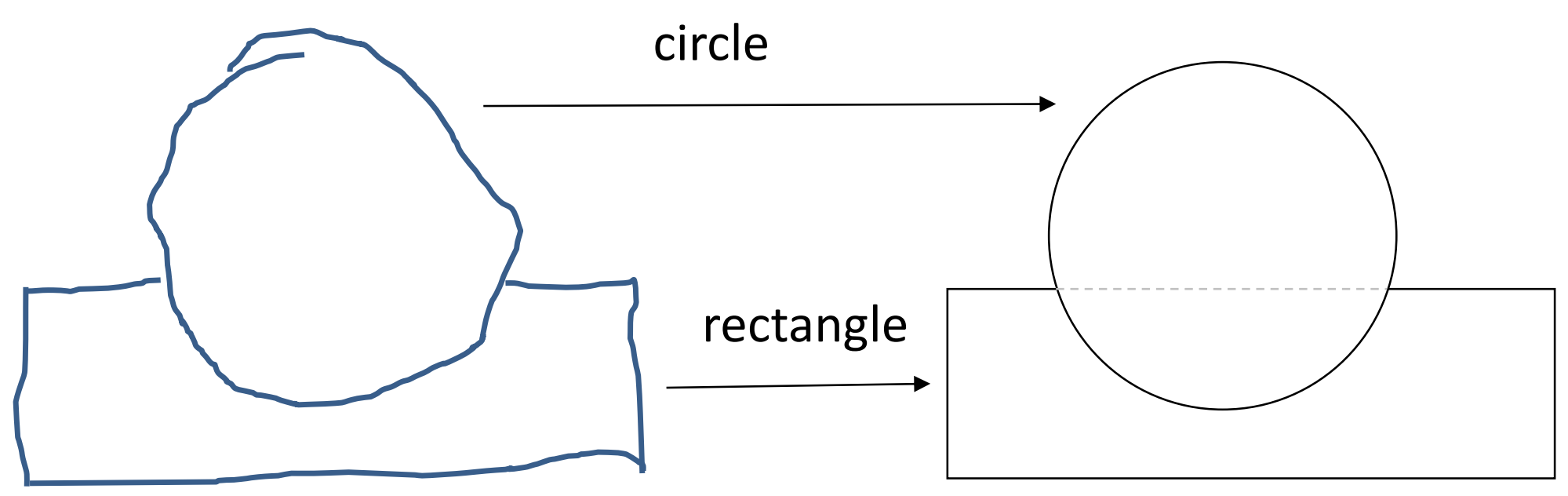




\section{Stroke Abstraction}

Stroke neatening that captures the essence of the stroke.
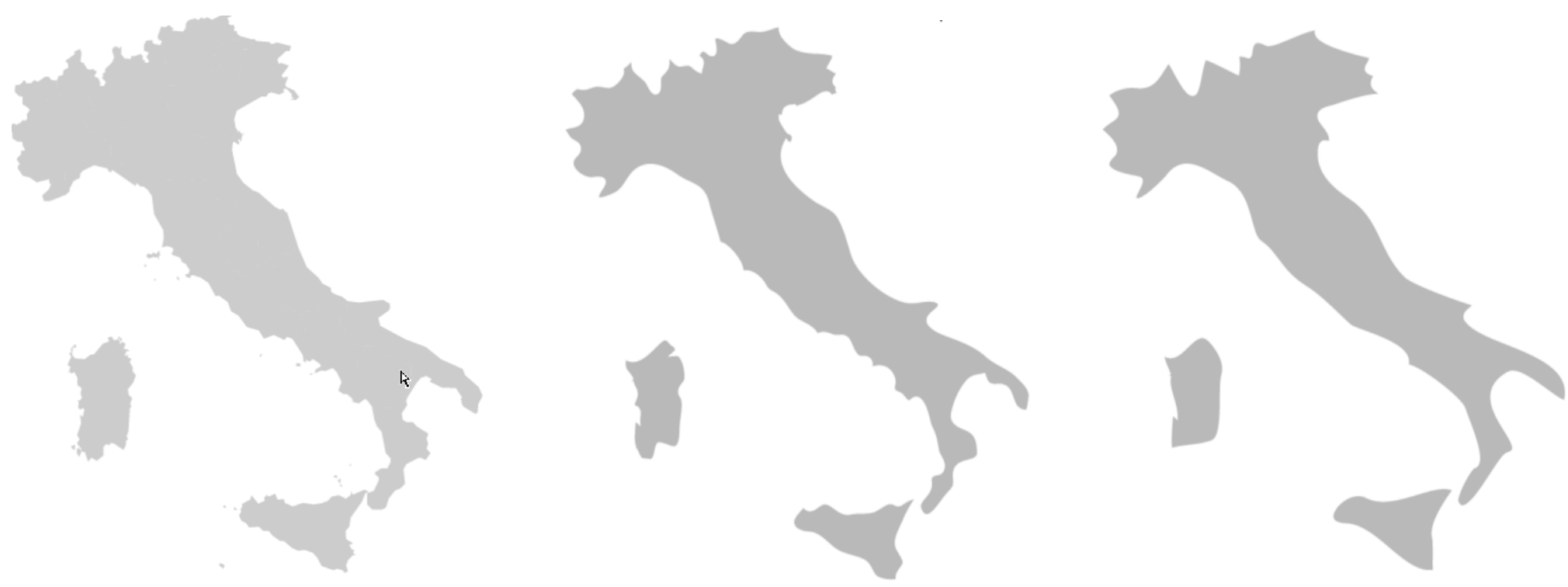

[X. Mi, D. DeCarlo and M. Stone Abstraction of 2D Shapes in Terms of Parts, NPAR 2009. ] 


\section{Stroke Appearance: NPR}

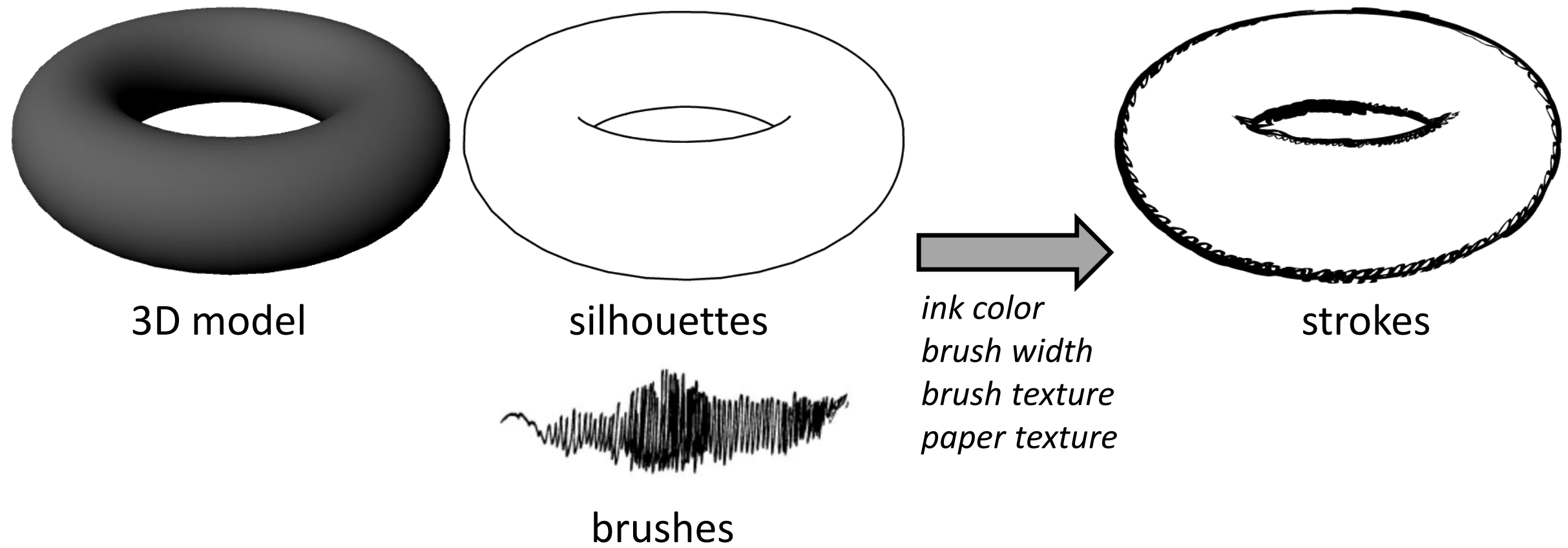




\section{Stroke Perception}

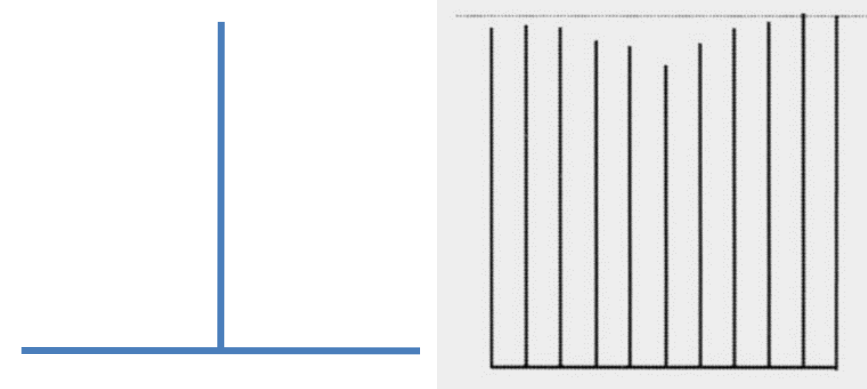

[Wolfe, Maloney \& Tam, Distortions of perceived length in the frontoparallel plane: tests of perspective theories, Perception \& pyschophysics, 2005]
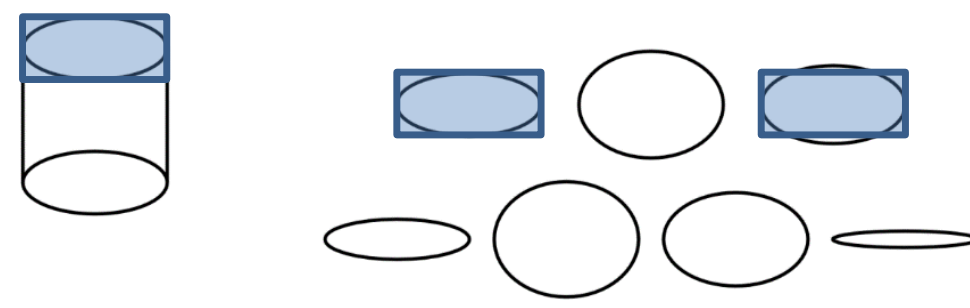

[Taylor \& Mitchell, Judgements of apparent shape contaminated by knowledge of reality: viewing circles obliquely, British Jnl. of Psych., 1997]
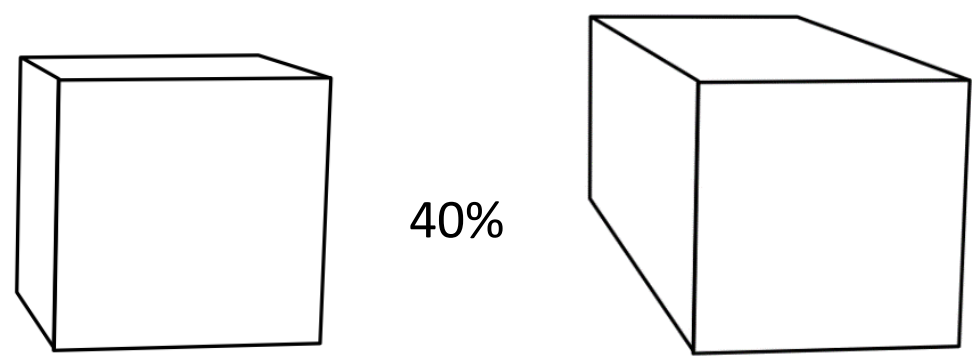

[Schmidt, Khan, Kurtenbach, Singh, On expert performance in 3D curve drawing tasks. SBIM 2009] 


\section{Stroke UI: crossing}
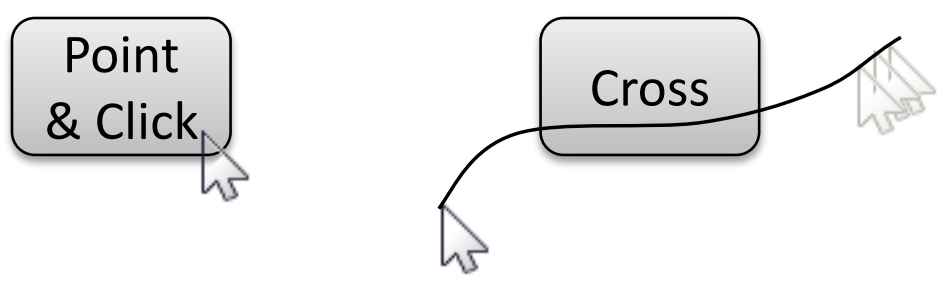

[Apitz, G. and Guimbretière, F. CrossY: A Crossing-Based Drawing Application ACM UIST, 2004] 


\section{Stroke UI: widgets}
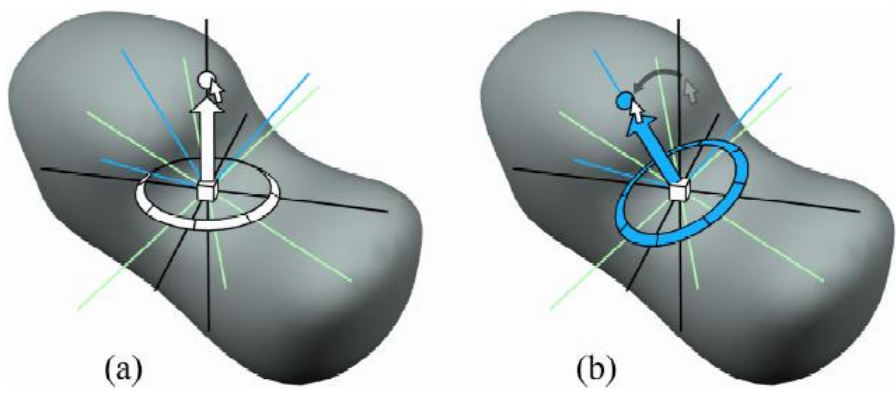

suggested axes

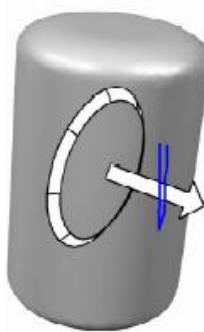

(a)

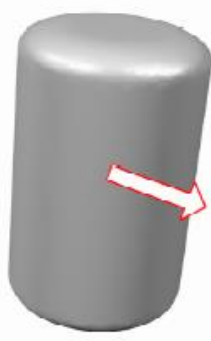

(b)

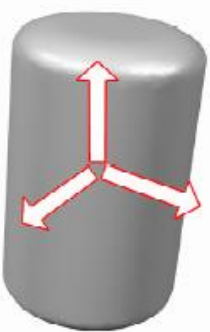

(c)

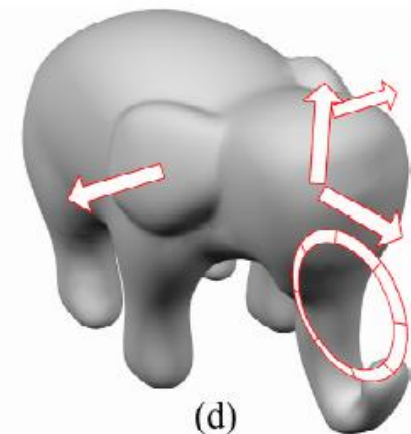

(d)

crossing interaction and composition

[Schmidt, Singh \& Balakrishnan Sketching and Composing Widgets for 3D Manipulation, Eurographics 2008] 


\section{Stroke UI: gestures}

- Ad-hoc or pre-defined:

- Application specific: shorthand, chinese Brush Painting, musical scores, chemical formulas.

- Platform specific: gesture libraries.

- Template-based:

- Toolkit or framework.

- Simple algorithm based on geometric matching. 


\section{Ad-hoc vs. template-based}

- Ad-hoc can recognize more complex gestures.

- Better consistency and collision handling of gestures in ad-hoc systems.

- Ad-hoc doesn't allow new gestures and limited customization.

- Template-based are easier for a user to specify and customize. 


\section{GRANDMA}

1. Encode gestures as a linear function of 13 features.

2. Draw a gesture 15 times.

3. Train asset of feature weights for each gesture.

4. Classify gestures based on highest feature function score.

[D. Rubine. Specifying gestures by example. SIGGRAPH 1991] 


\section{\$1 recognizer goals}

- Resilience to sampling.

- Require no advance math.

- Small code.

- Fast.

- 1-gesture training.

- Return an N-best list with scores.

[J. Wobbrock, A. Wilson \& Y. Li. 2007. Gestures without libraries, toolkits or training: a \$1 recognizer for user interface prototypes. ACM UIST '07.] 


\section{\$1 algorithm}

- Resample the input

- N evenly spaced points

- Rotate

- "Indicative" angle between centroid and start point

- Scale

- Reference square

- Re-rotate and Score

- Score built from average distance between candidate and template points 


\section{\$1 limitations}

- Cannot distinguish aspect ratios, orientations.

- Square from rectangle

- Up arrow from down arrow

- Cannot be distinguished based on speed.

- Only single strokes.

- Stroke order is important.

- Closed strokes?

- Gestalt gestures! 


\section{Take-aways}

- Understand your application:

- Does it need strokes?

- Are strokes natural and of low-complexity, 2D or 3D?

- Understand source of stroke error before filtering?

- Ensure users can control stroke dynamics before you exploit it.

- Both clean and sketchy stroke appearances are useful.

- Understand perceptual bias in drawn strokes.

- Develop a GUI suited to stroke interaction. 
Multi-view sketch-based modeling of 3D curves and surfaces

\section{Yotam Gingold}


How can we turn sketch strokes into 3D shapes?

- Interpreting them as gestures

- Interpreting them as silhouettes

- Projecting them in 3D

- General principle: Drawing from different points of view. 


\section{Interpreting stokes as gestures}




\section{SKETCH}

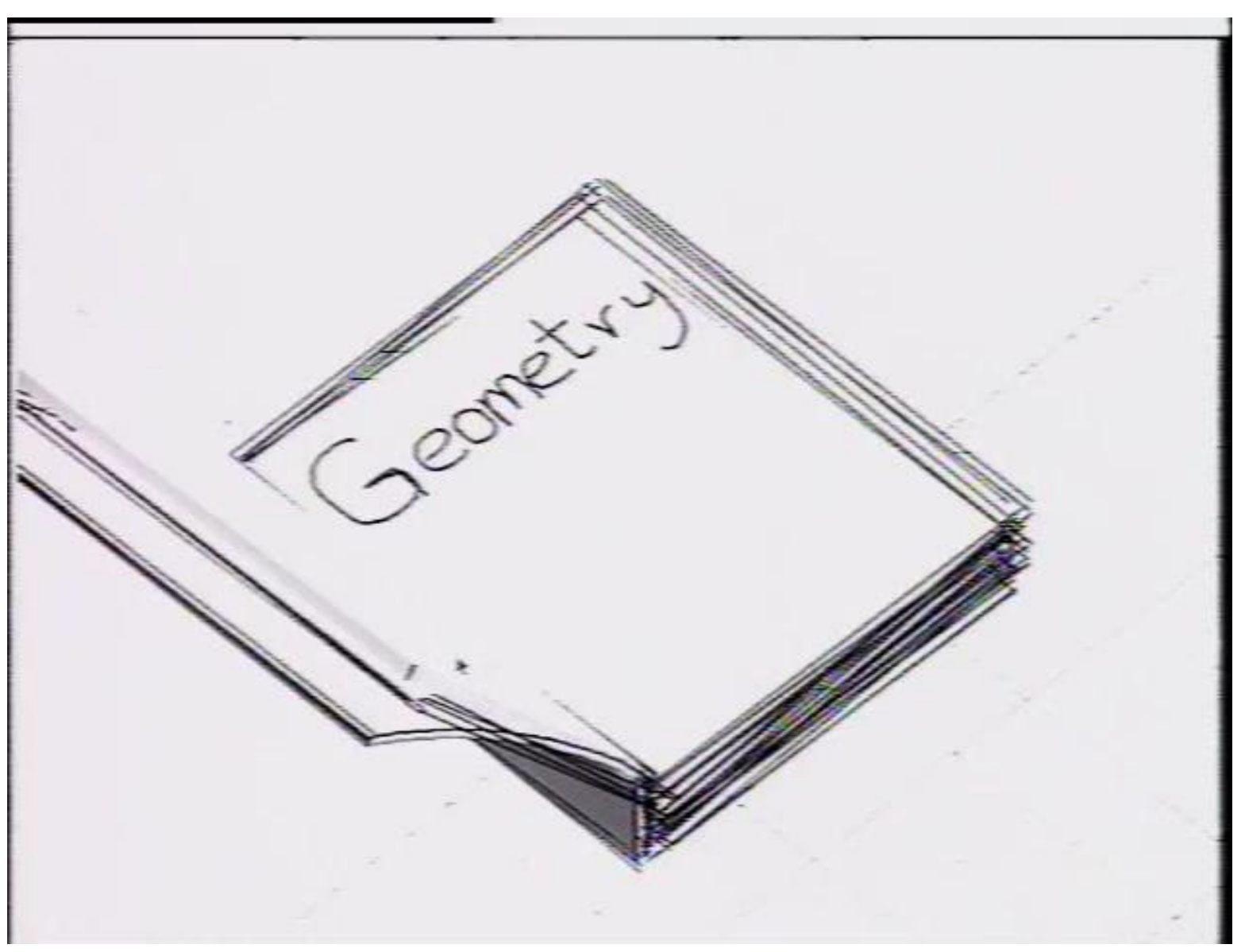

SKETCH: An Interface for Sketching 3D Scenes [Zeleznik et al. 1996] 
Interpreting strokes as silhouettes 


\section{Teddy}

\section{Takeo Igarashi Hidehiko Tanaka University of Tokyo Satoshi Matsuoka Tokyo Institule of Technology}

Teddy:

A Sketching

Interface for 3D

Freeform Design

Teddy: A Sketching Interface for 3D Freeform Design [Igarashi et al. 1999] 


\section{More freeform inflation approaches}

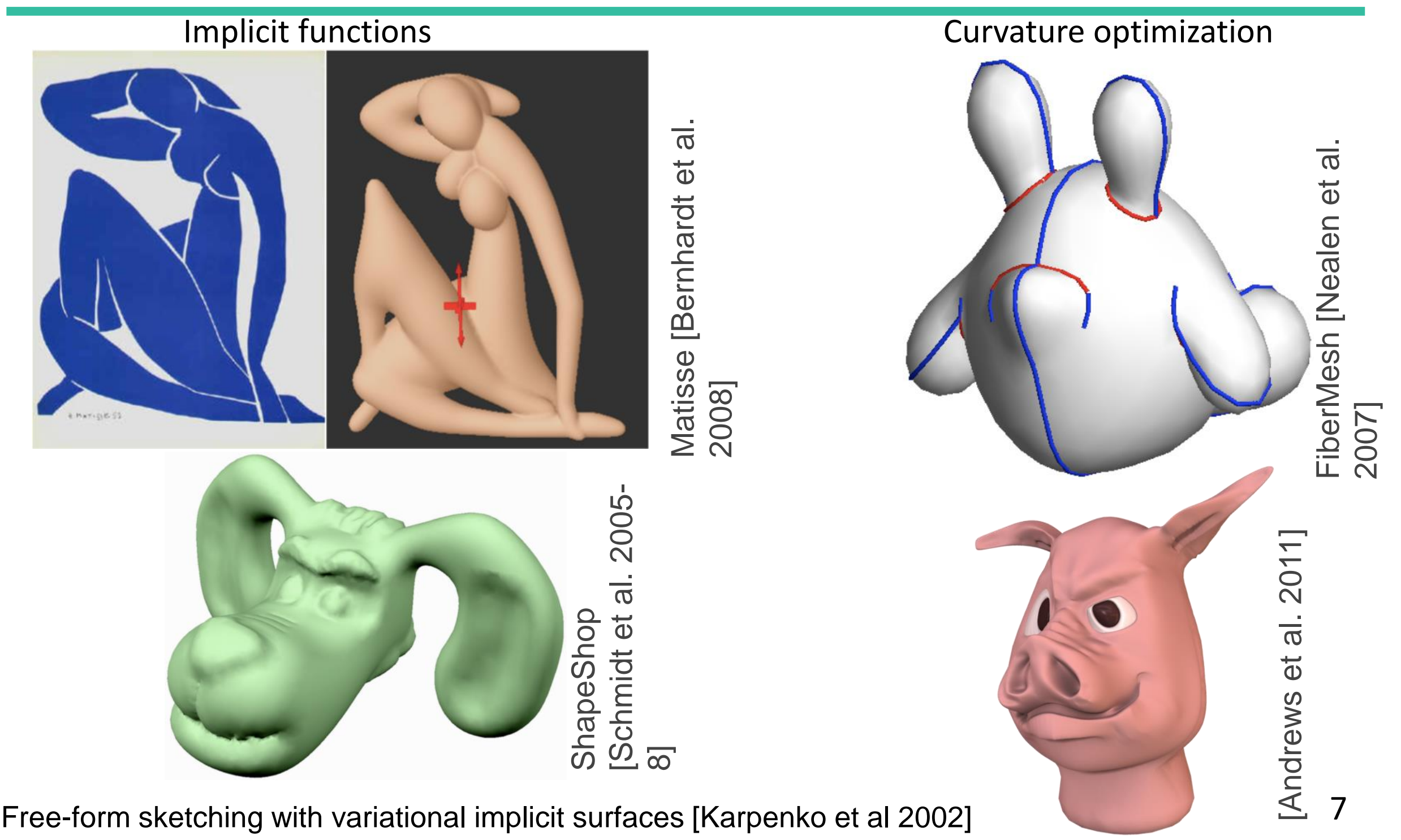




\section{Inflation (Teddy)}

- Step 1
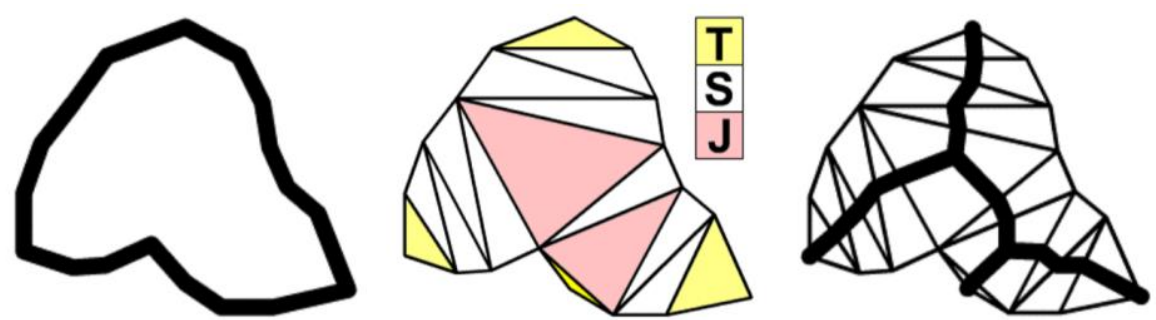

a) initial $2 \mathrm{D}$ polygon

b) result of CDT

c) chordal axis
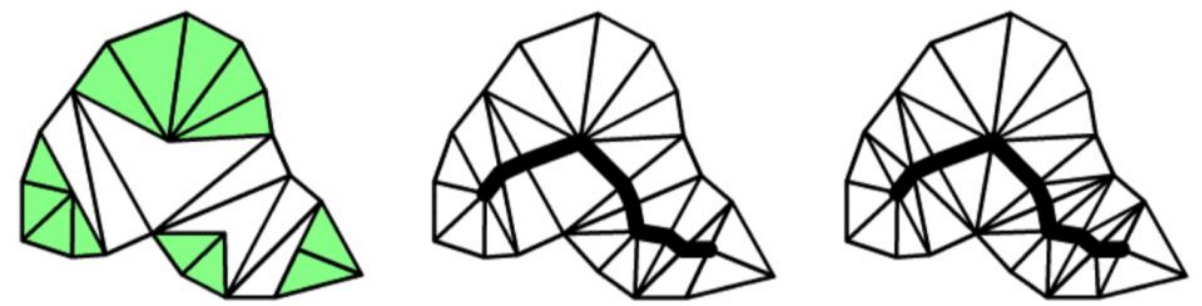

d) fan triangles

e) resulting spine

f) final triangulation

- Step 2
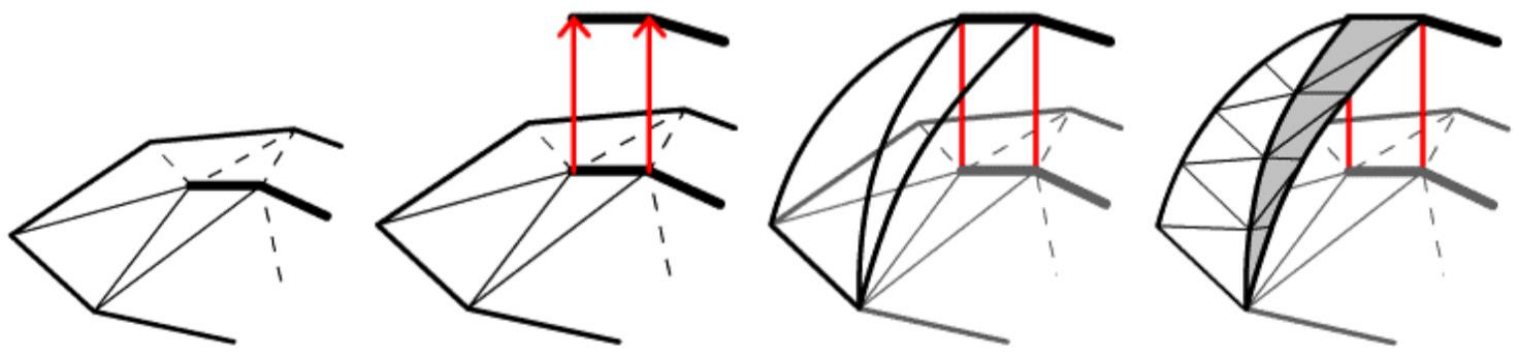

a) before b) elevate spines

c) elevate edges d) sew elevated edges 


\section{Extrusion (Teddy)}
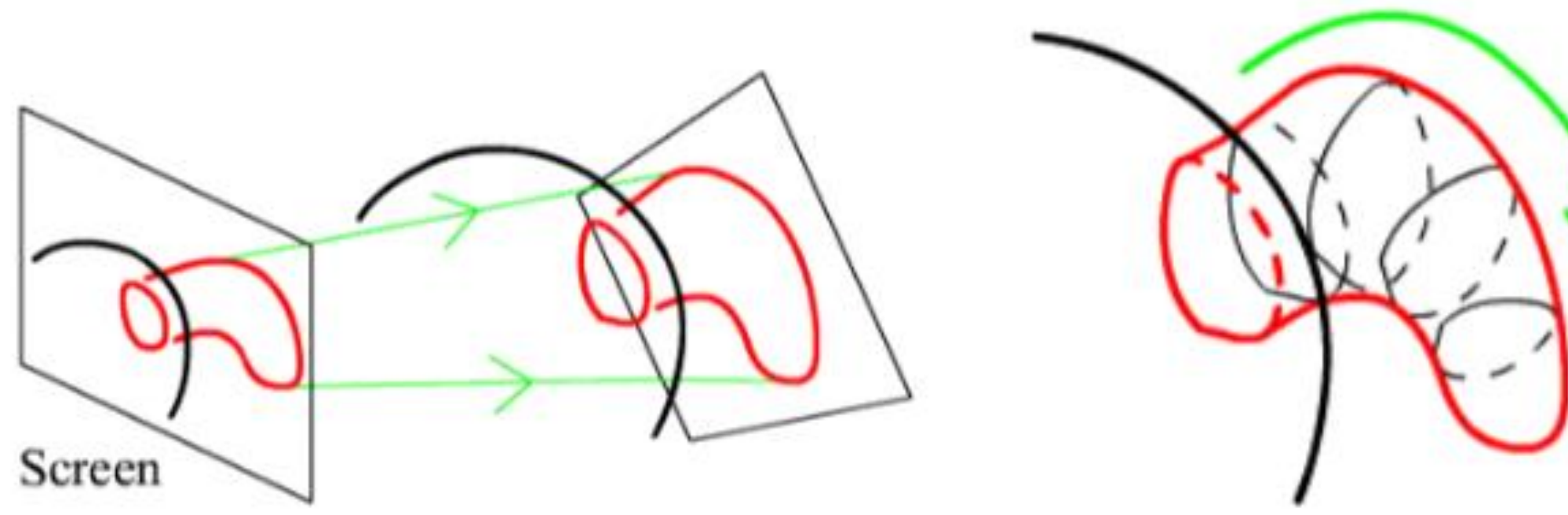

a) projection of the stroke

b) sweep along the projected stroke 


\section{Inflation (implicit surface)}
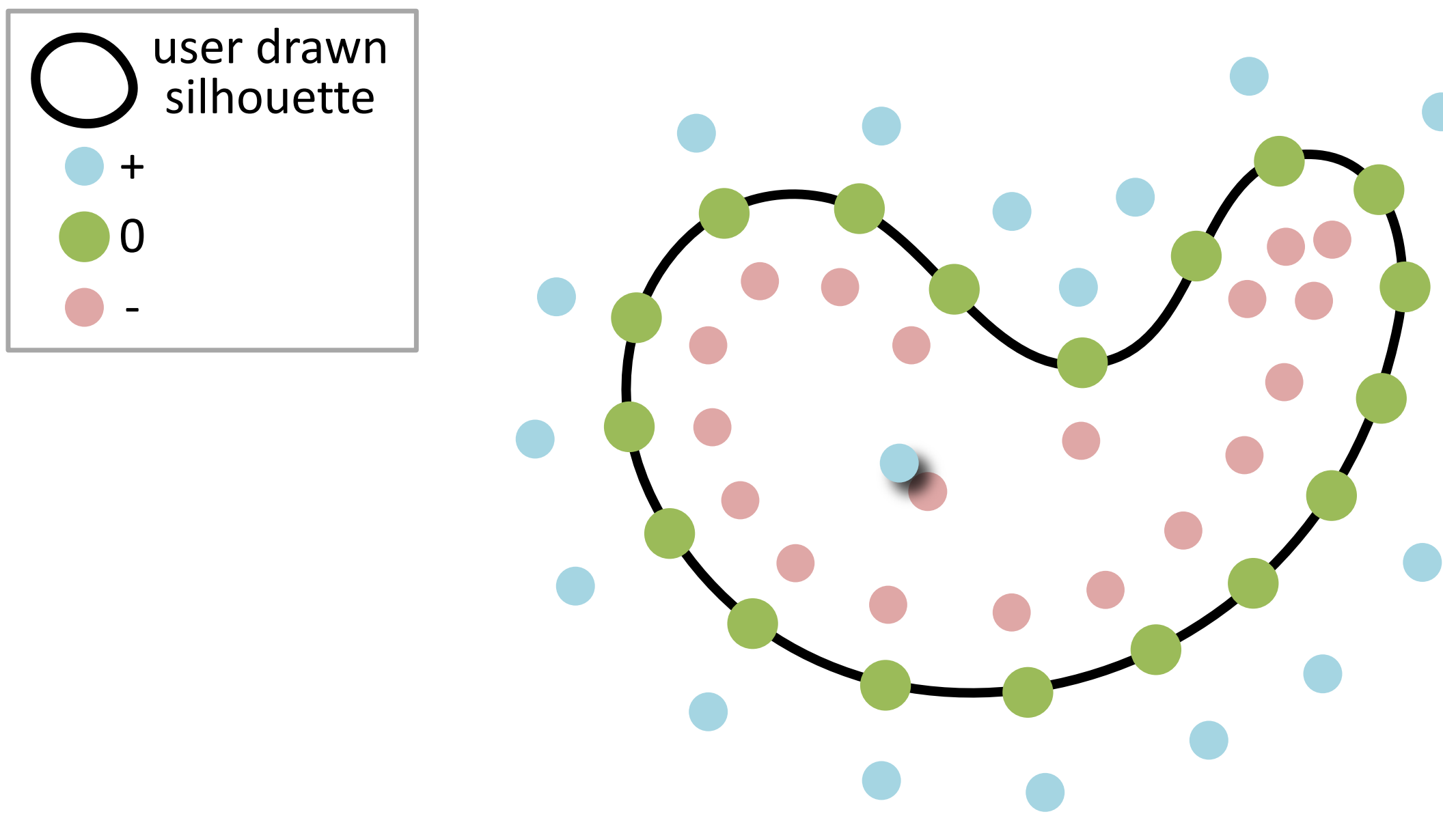

QQ, SIGGRAPH

ASIA 2016 


\section{Inflation (surface optimization)}

initial mesh

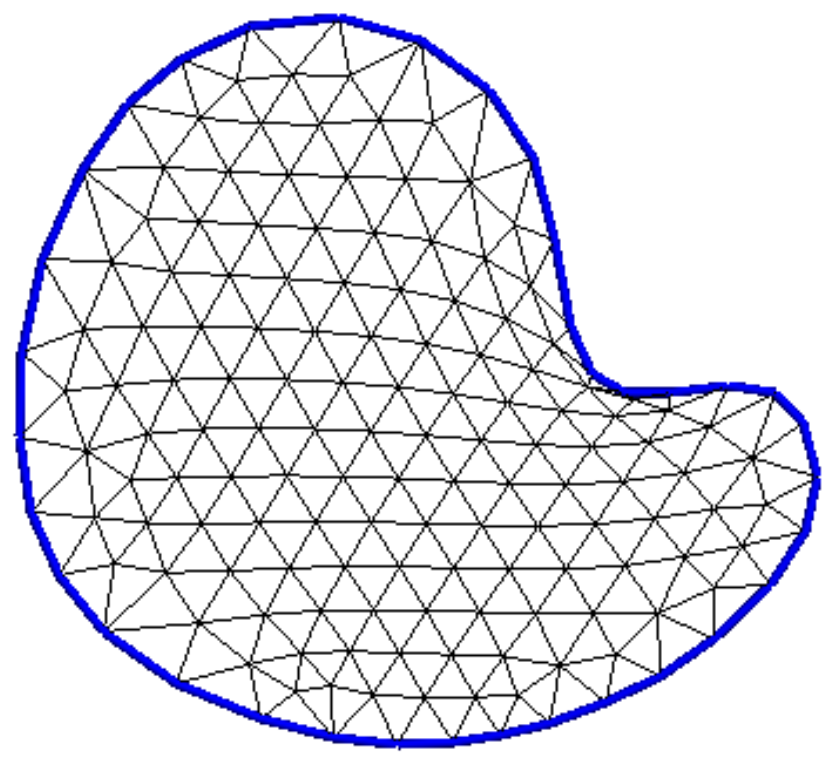

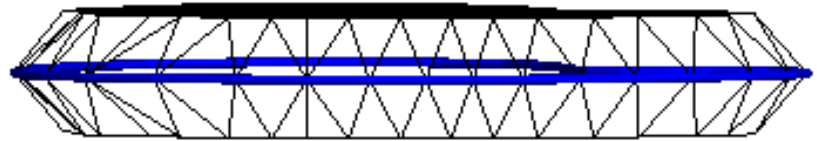

minimum variation of curvature
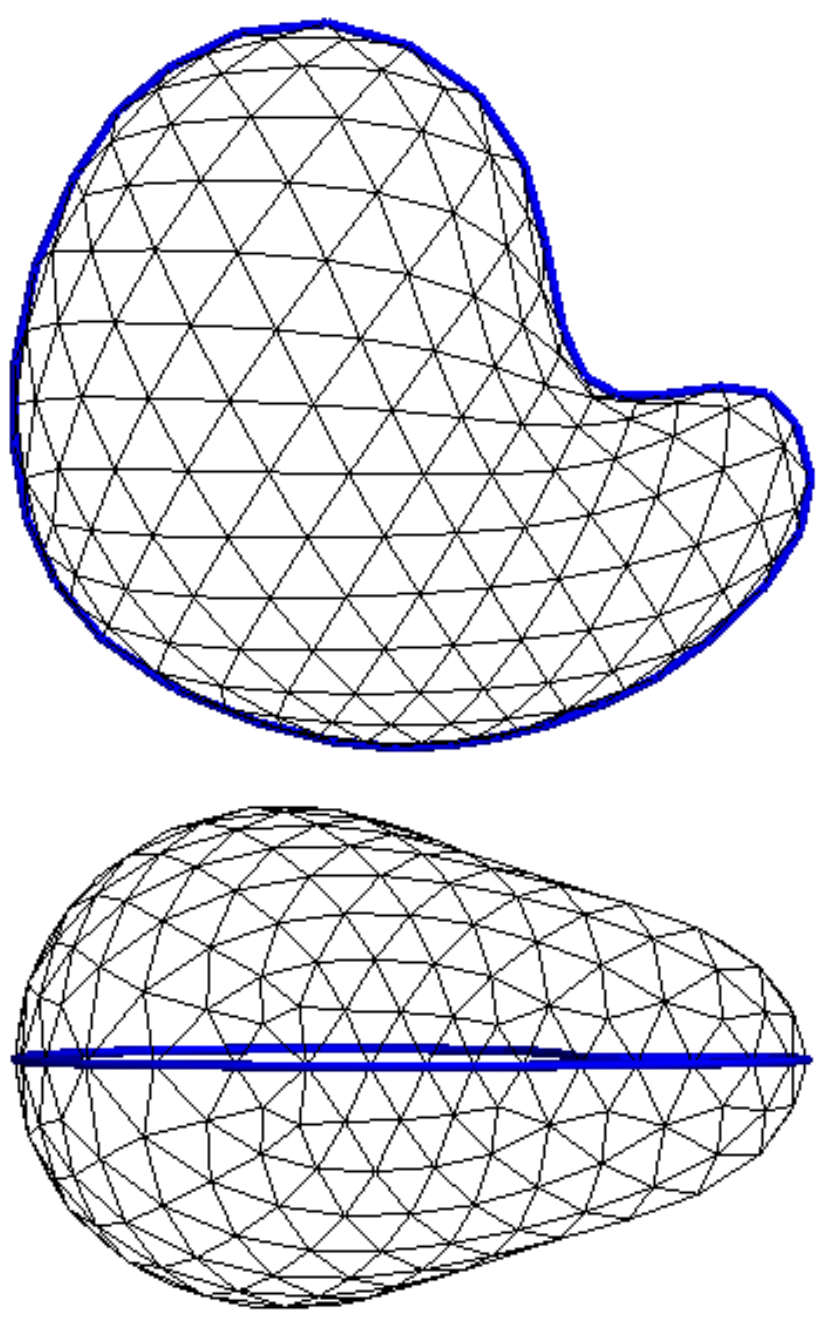


$$
\overline{0} \overline{0}
$$




\section{Cutting (Teddy)}

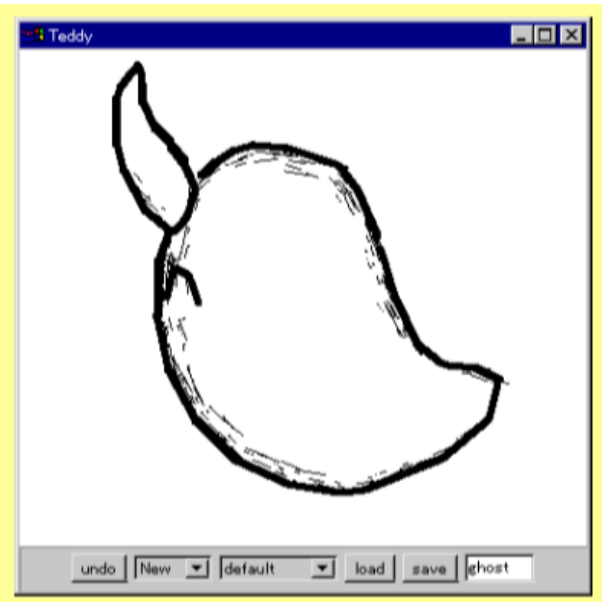

Before

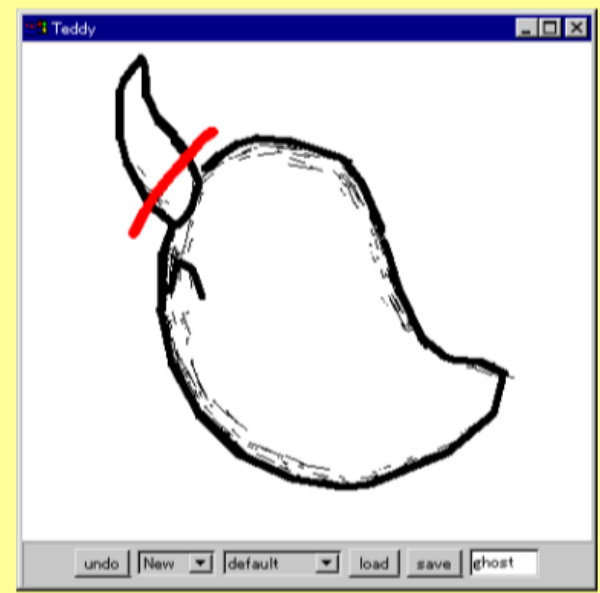

Cutting stroke
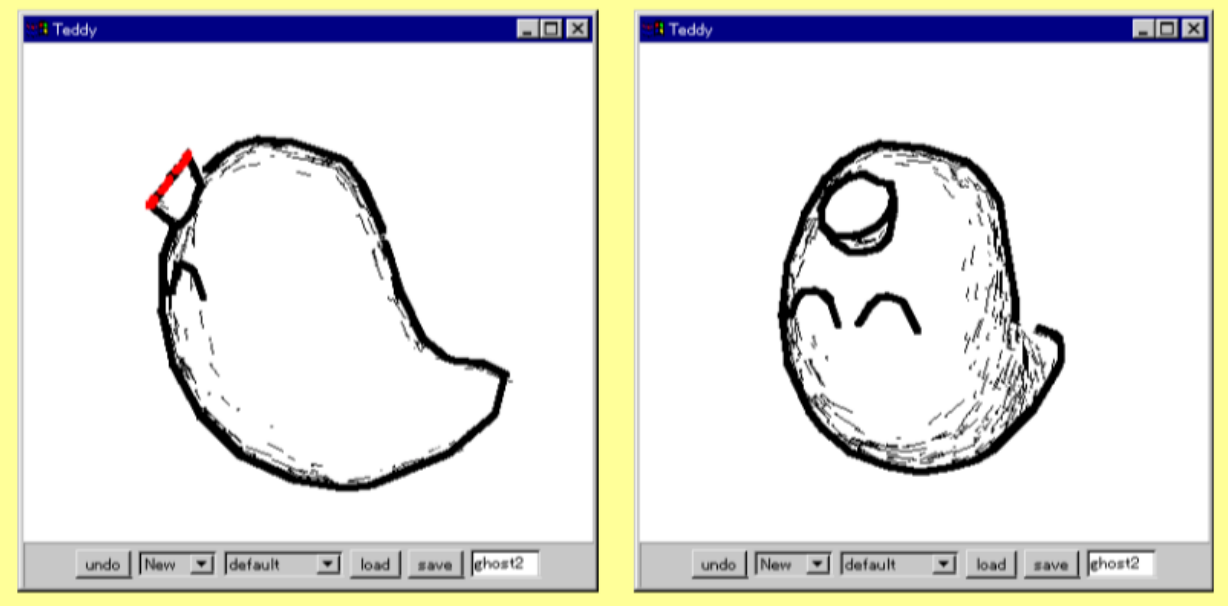

After 
Projecting strokes in 3D 


\section{Ambiguity}

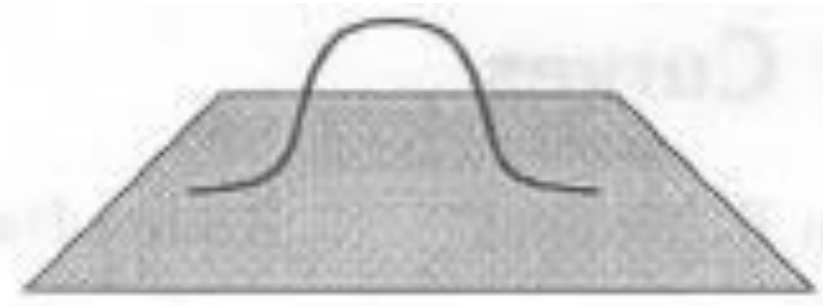

Figure 1: A single stroke creates the initial curve.

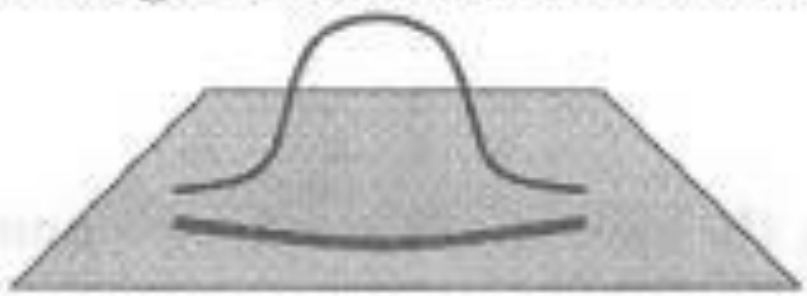

Figure 2: A second stroke defines the curve's shadow and hence its $3 \mathrm{D}$ shape.

An Interface for Sketching 3D Curves [Cohen et al. 1999] 


\section{Ambiguity}

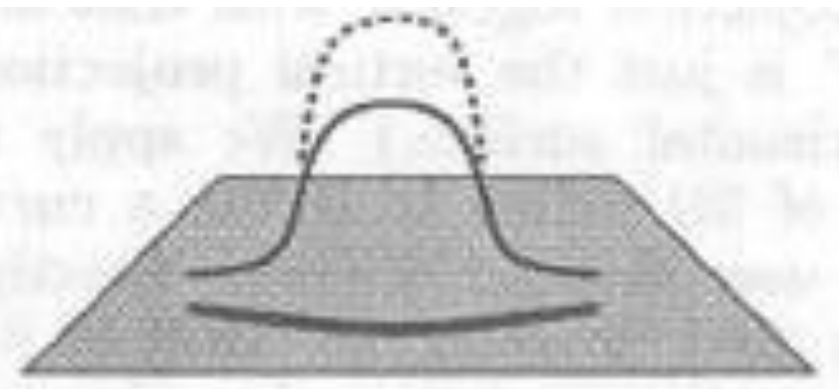

Figure 3: The dashed line indicates an overdraw stroke.

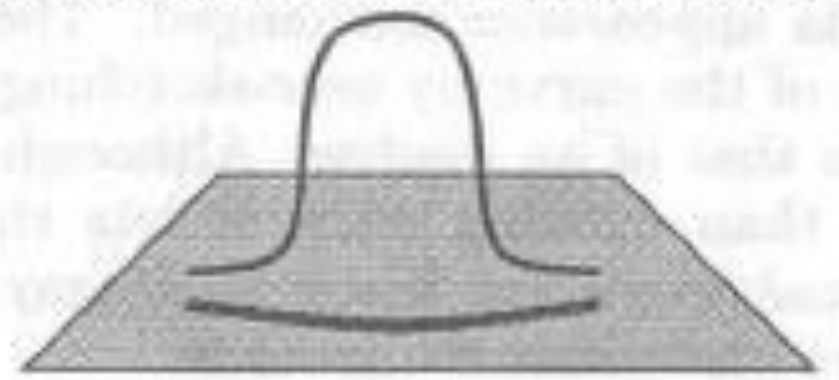

Figure 4: The system blends the overdraw with the original curve to get the final result.

An Interface for Sketching 3D Curves [Cohen et al. 1999] 


\section{Ambiguity}

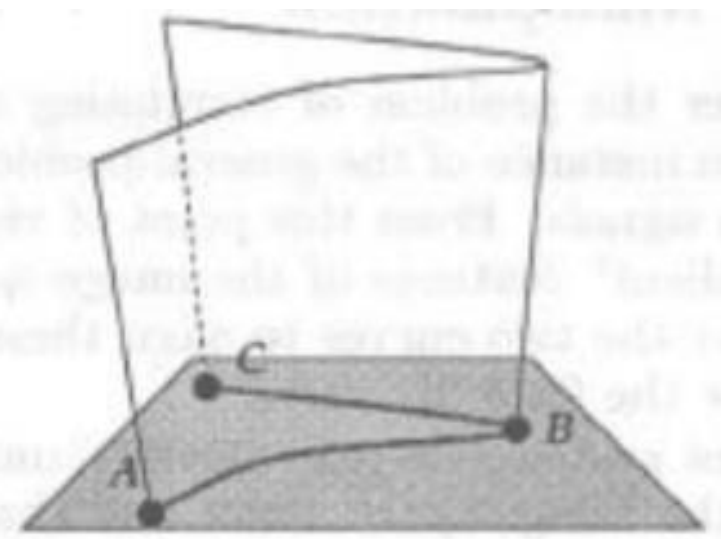

Figure 7: The shadow defines a ruled surface with a silhouette above the interior critical point of the shadow, $B$.

An Interface for Sketching 3D Curves [Cohen et al. 1999] 


\section{Ambiguity}

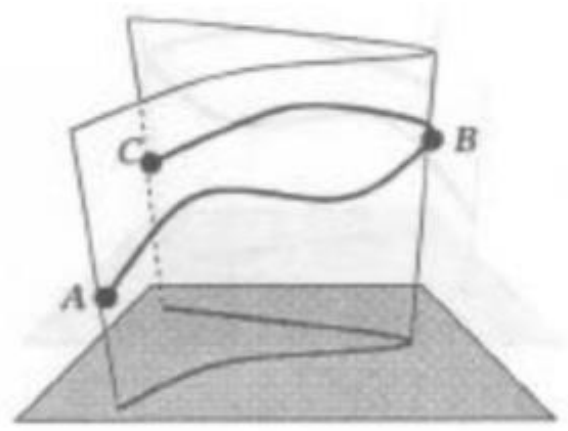

Figure 8: The curve must turn around at $B$ to stay on the surface.

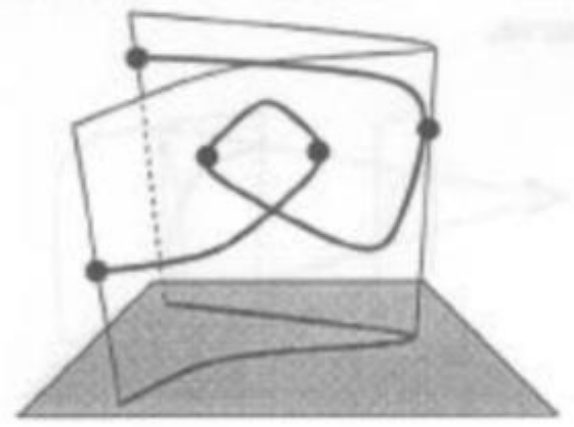

Figure 9: The curve may have more critical points than the shadow and still be valid.

An Interface for Sketching 3D Curves [Cohen et al. 1999] 


\section{Ambiguity}

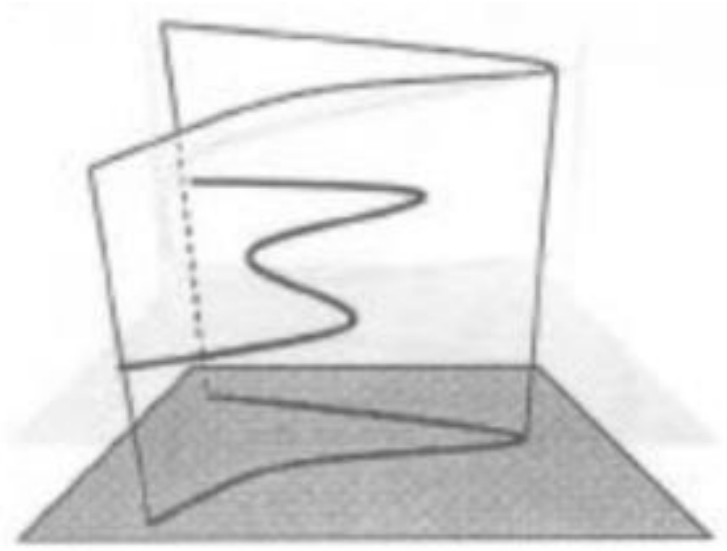

Figure 10: There is no way to project this curve onto the surface to get a continuous $3 \mathrm{D}$ curve.

An Interface for Sketching 3D Curves [Cohen et al. 1999] 


\section{iLoveSketch}

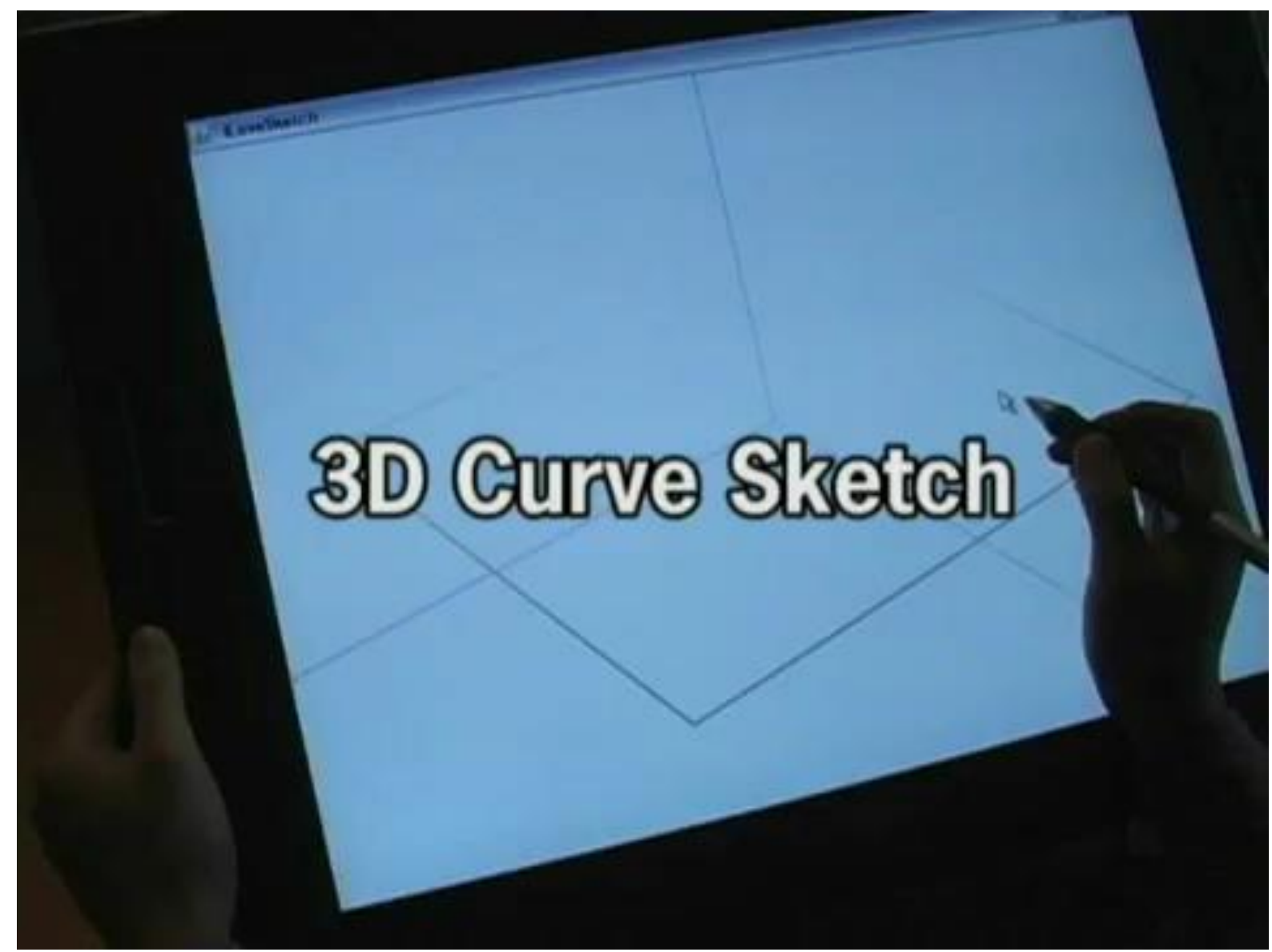

iLoveSketch: As-natural-as-possible sketching system for creating 3D curve models [Bae et al 2008] Q19, SIGGRAPH

ASIA 2\$16 


\section{Takeaways}

- We can remove the ambiguity in depth in several ways:

- with assumptions (rotund surfaces)

- by projecting onto other surfaces

- by sketching from multiple points of view 


\title{
Sketch-based modelling using prior knowledge
}

\author{
Marie-Paule Cani
}

Grenoble University \& Inria 


\section{Use of prior knowledge : Motivation}
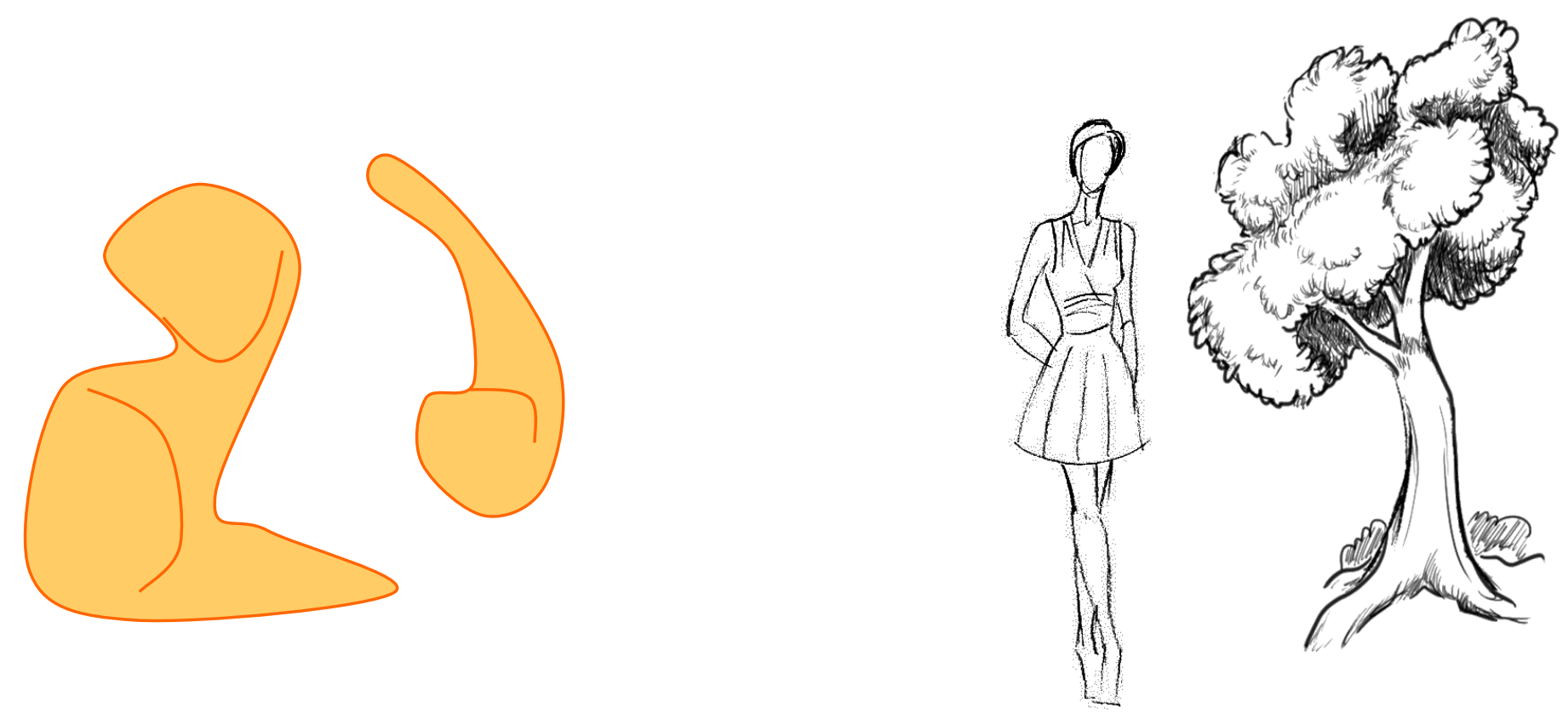

Why do we "see" 3D shapes when we look at a sketch? 


\section{Use of prior knowledge : Motivation}

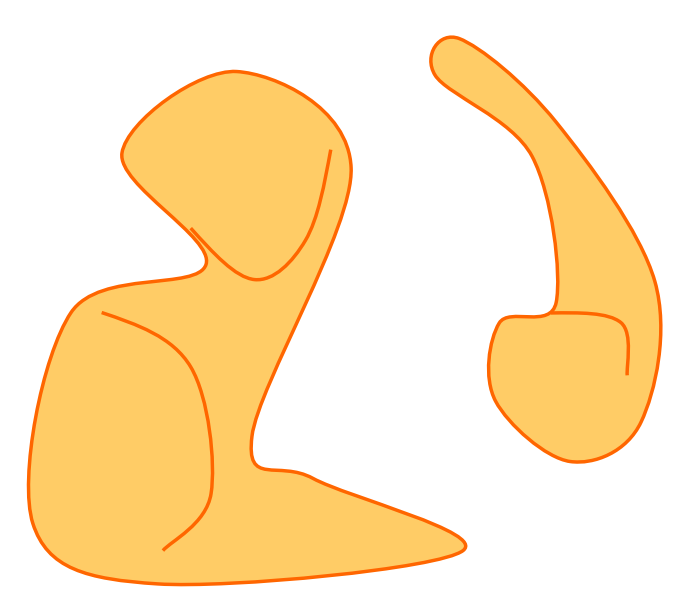

Unknown shape

- We infer the simplest one

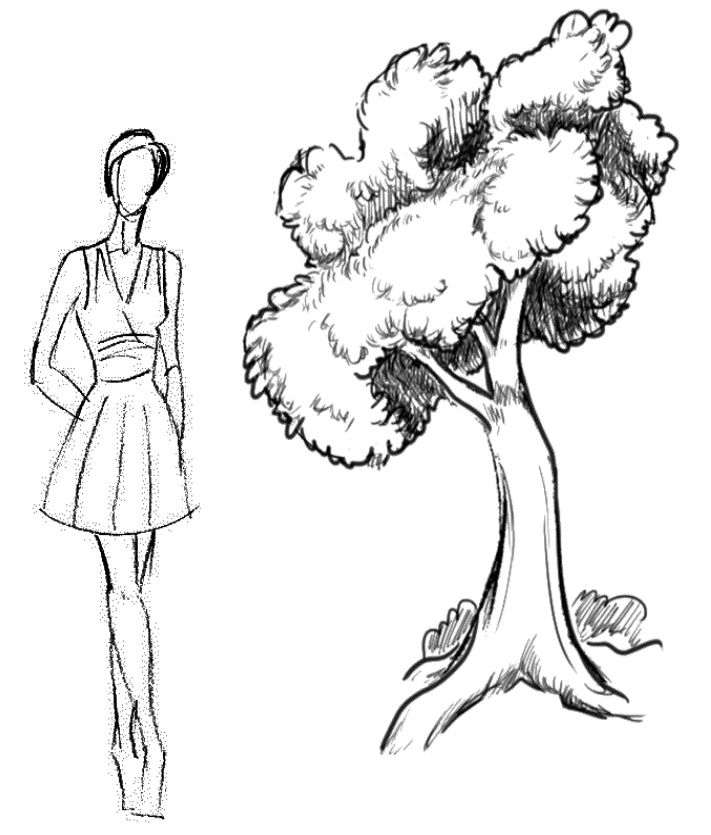

Well known shapes

- We use prior knowledge 


\section{Use of prior knowledge : Motivation}

Well known shapes

- Model prior knowledge

- Use it to fill in the missing data

Benefits

- 3D models from a single sketch !

- Constraints maintained!

In this talk

- Animals, trees and garments

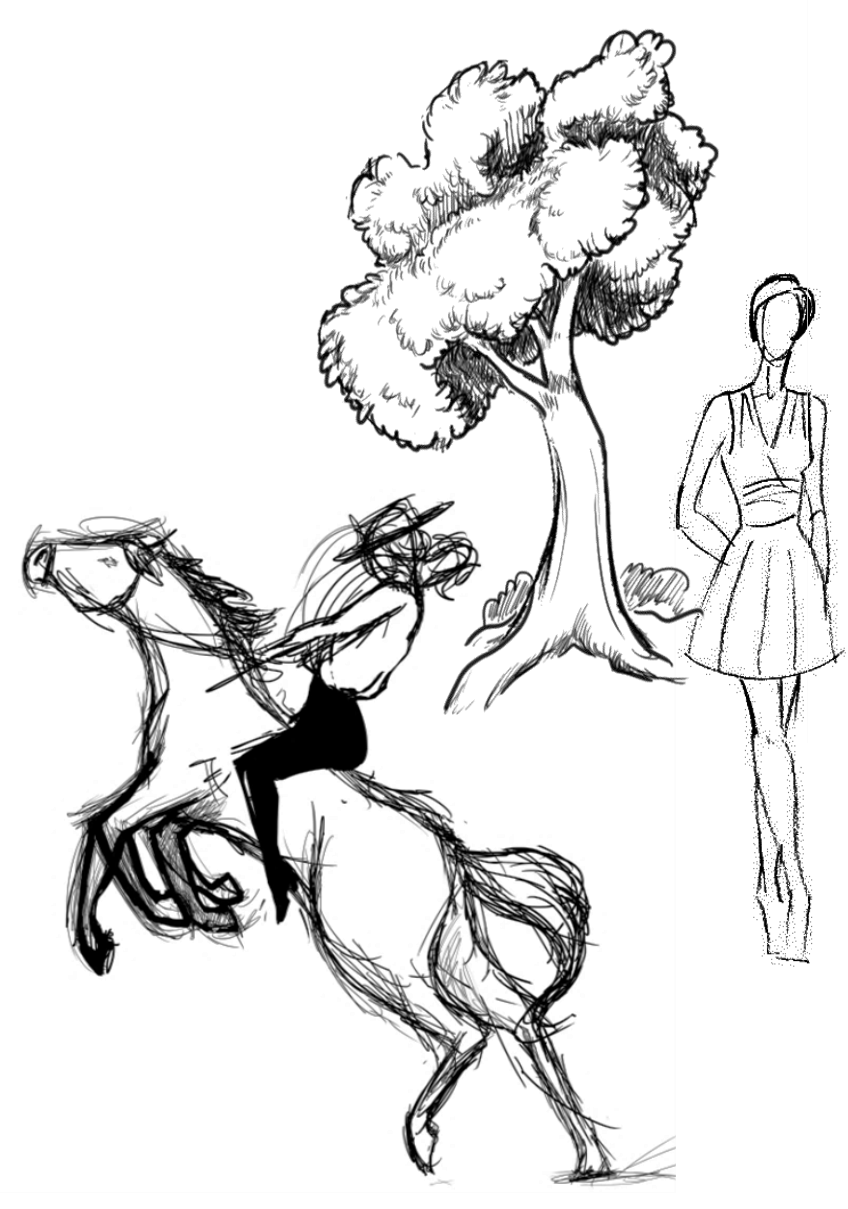




\section{Sketching specific shapes \\ Example 1: Animals from a side-view sketch}

Goal: Modeling animals and other creatures from a single sketch

Side view

Vector drawing
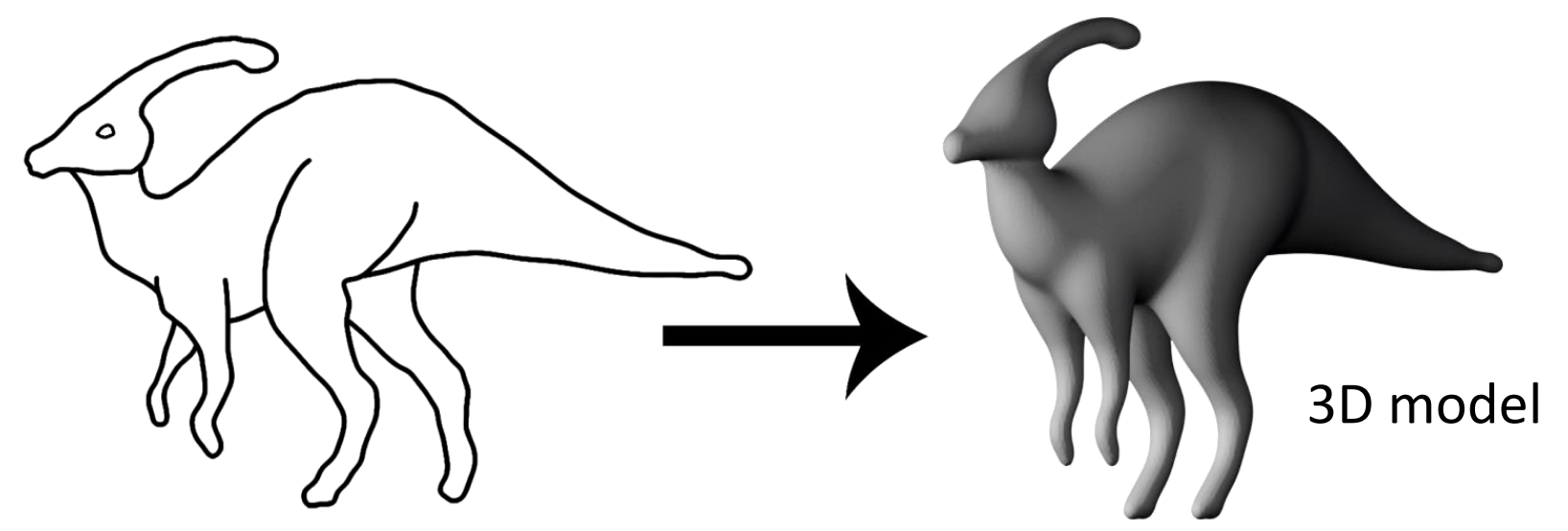

\section{A priori knowledge}

- Organic shapes: Mostly smooth, volumetric shapes

- Anatomic principles : sagittal plane symmetry, pairs of limbs

- Structural symmetry of the sketch (limbs in arbitrary postures) 


\section{Animals from a side-view sketch Choice of 3D shape representation}

- Polygon meshes inflated from contours

?

$\sim$ Enables flat areas and full control of geometry

- Post-editing is not user-friendly

- Skeleton-based implicit surfaces ?

+ Smooth, volumetric shapes

+ Easy progressive blending

$+3 D$ line skeletons suited for post-editing

[Bernhardt et al., SBIM 2008]
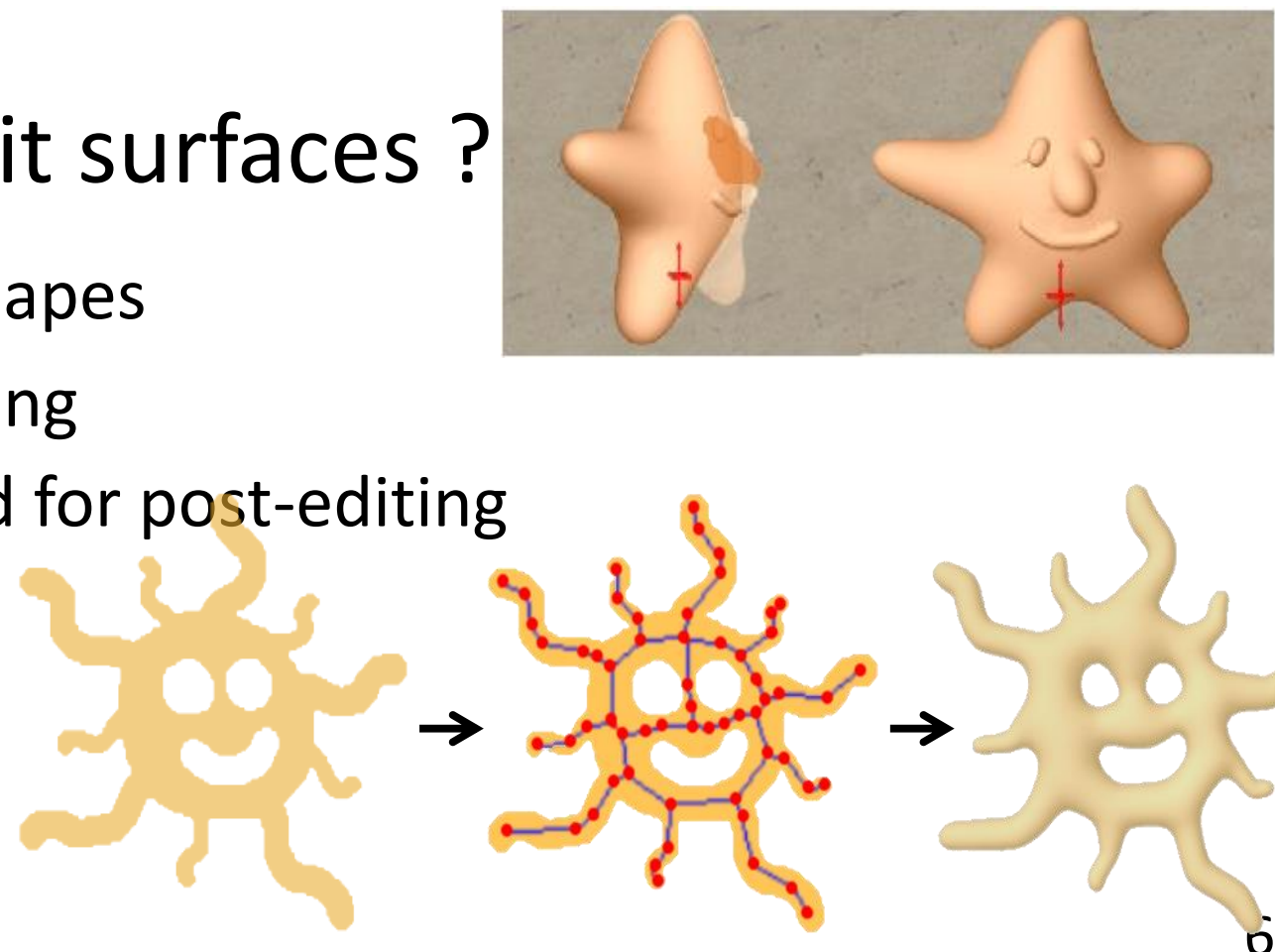


\section{Animals from a side-view sketch Choice of 3D shape representation}

Implicit surface $S=\{P / f(P)=c\}, \quad f(P)=\Sigma f_{i}(P)$
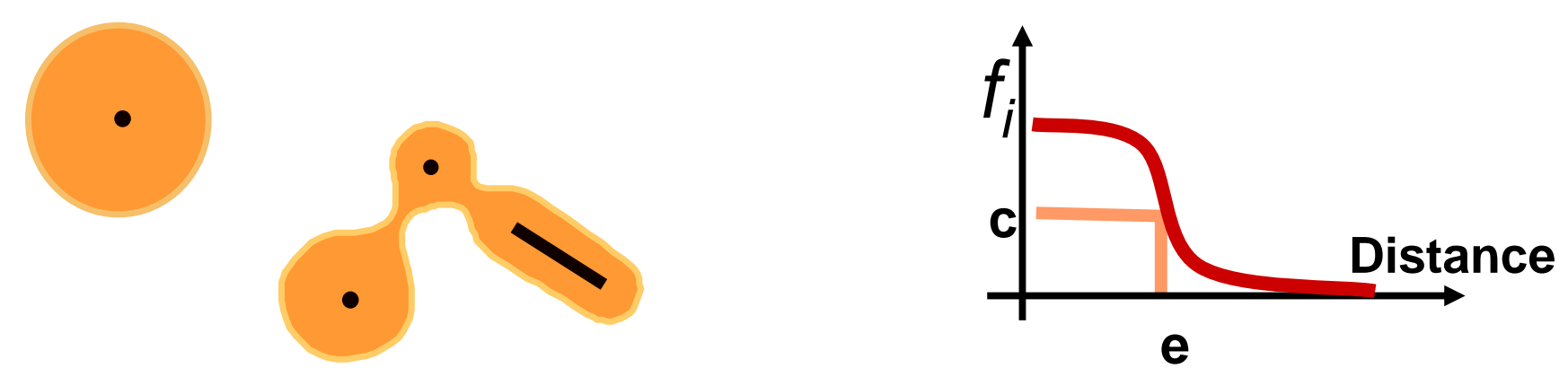

SCALIS: SCALe Invariant integral Surfaces

- Extension of convolution surfaces

- Scale invariant

- Exact contour matching

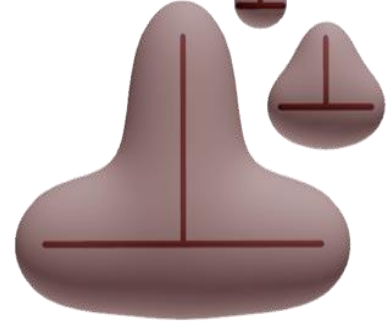

(a) Standard

[Zanni Bernhardt Quiblier Cani

CGF2013]

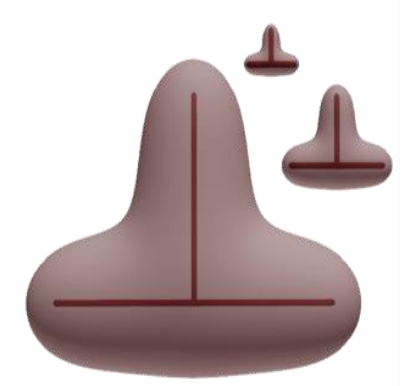

(c) SCALIS 


\section{Animals from a side-view sketch Overview of the method}

1. Sub-parts detection, contours closing

2. Using structural symmetries to clean the sketch

3. Depth positioning and implicit reconstruction

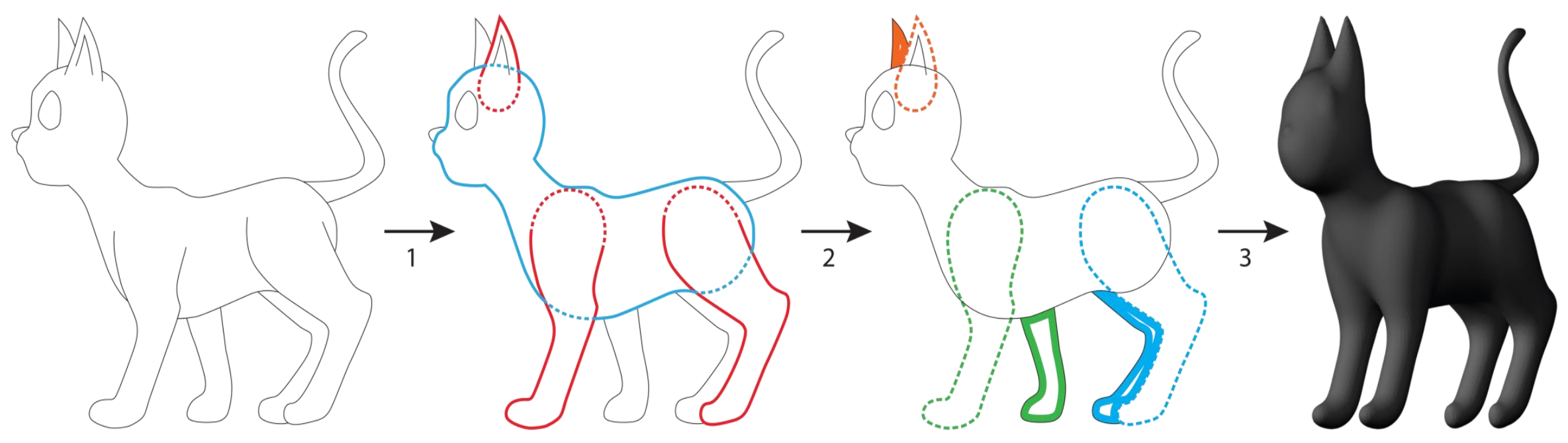




\section{Animals from a side-view sketch \\ 1. Identifying subparts from the sketch}

Perceptual process

- Subparts are partially/fully bounded by curves

- They may be partially occluded

- Depth ordering from cues ("T-junctions" and inclusions)

-> Semantic curve classification

- Silhouette contours

- Suggestive contours (red)

$\rightarrow$ Internal silhouettes 


\section{Animals from a side-view sketch \\ 2. Cleaning the sketch}

\section{Knowledge on structural symmetries}

- Standing pose hypothesis: pairing based on angle

- Enables to remove background limbs
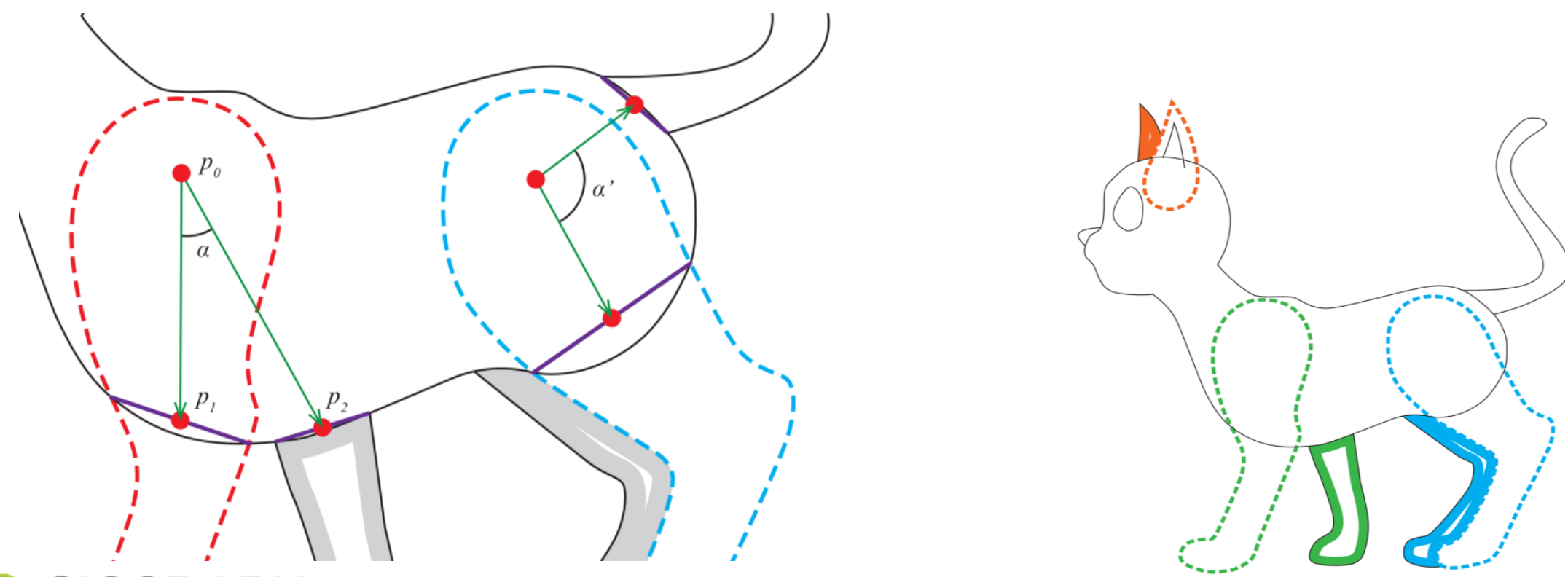


\section{Animals from a side-view sketch 3. Generating the implicit 3D model}

- Skeletons of shape-parts : Medial-Axis

- 3D parts : SCALIS surfaces

- Relative depths from a priori knowledge

- "flesh around bones" hypothesis

- Blending

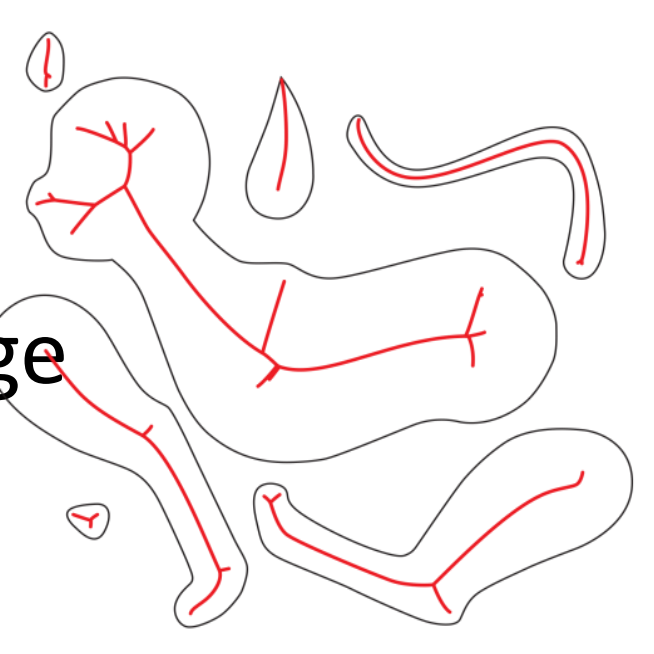

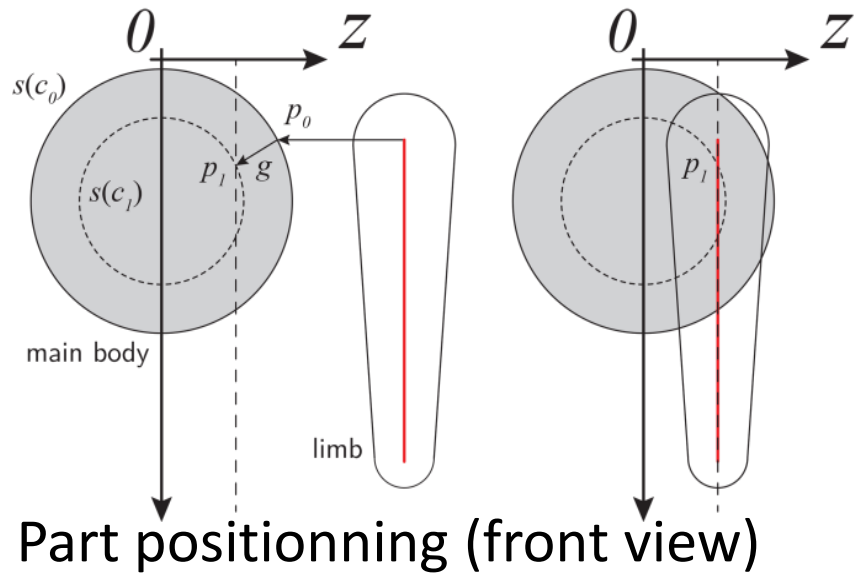

Part positionning (front view)

Q8, SIGGRAPH - ASIA 2516

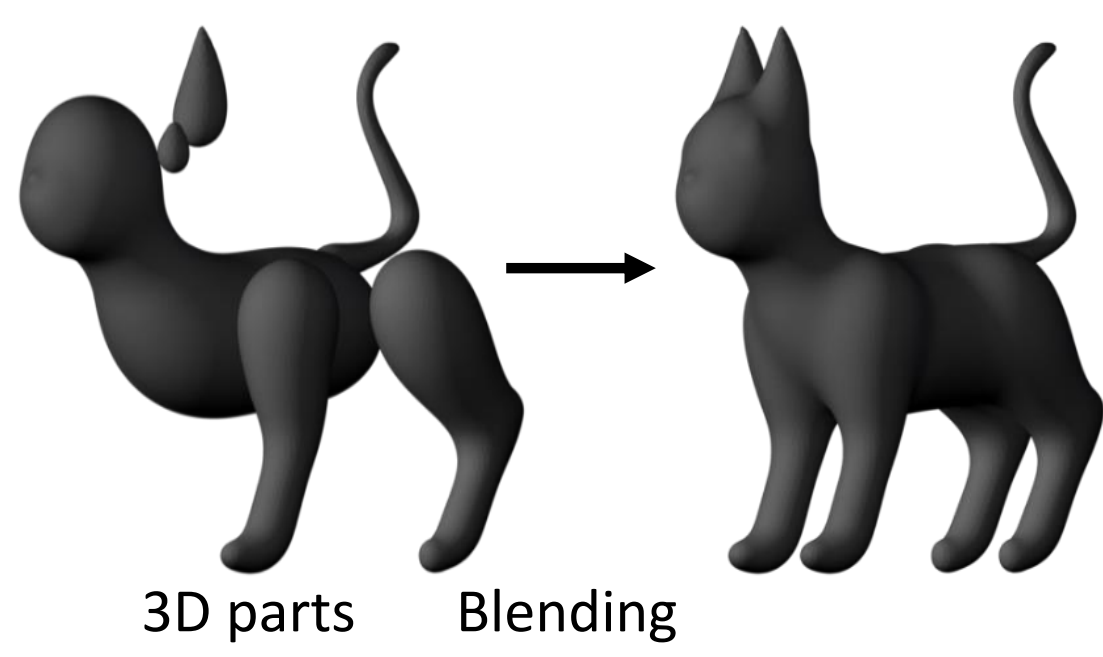




\section{Animals from a side-view sketch}
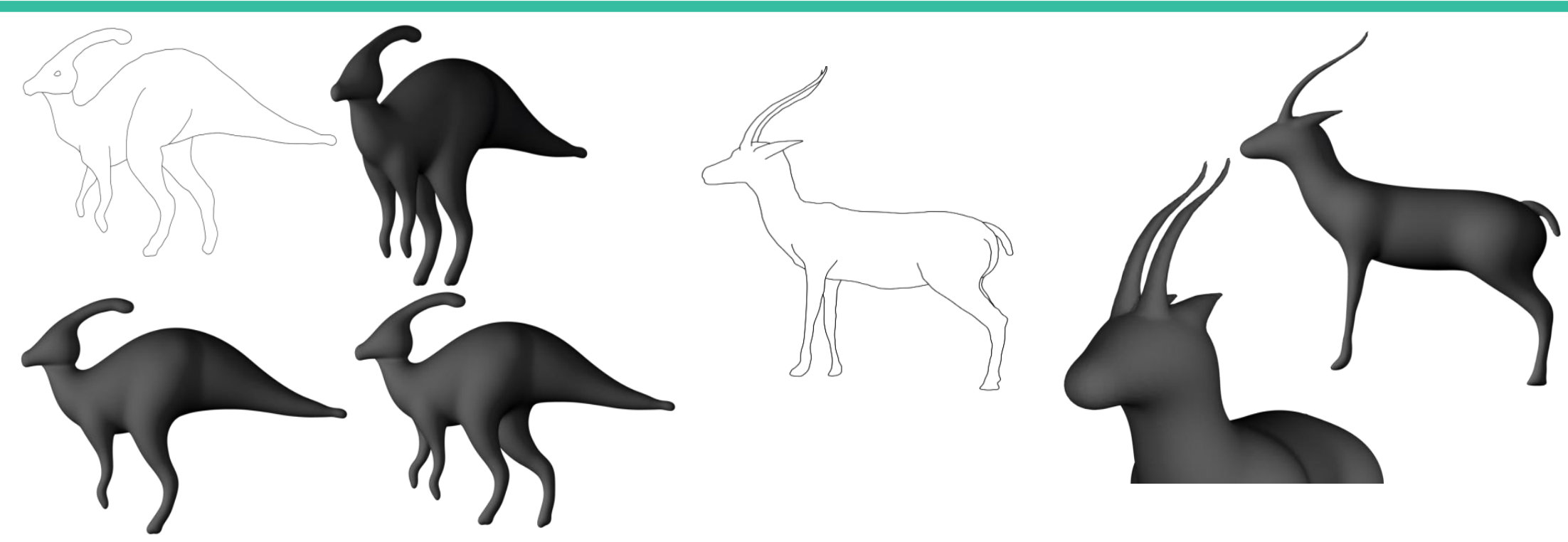

[Entem, Barthe, Cordier, Cani, Van de Panne, SMI'2014] Q?, SIGGRAPH

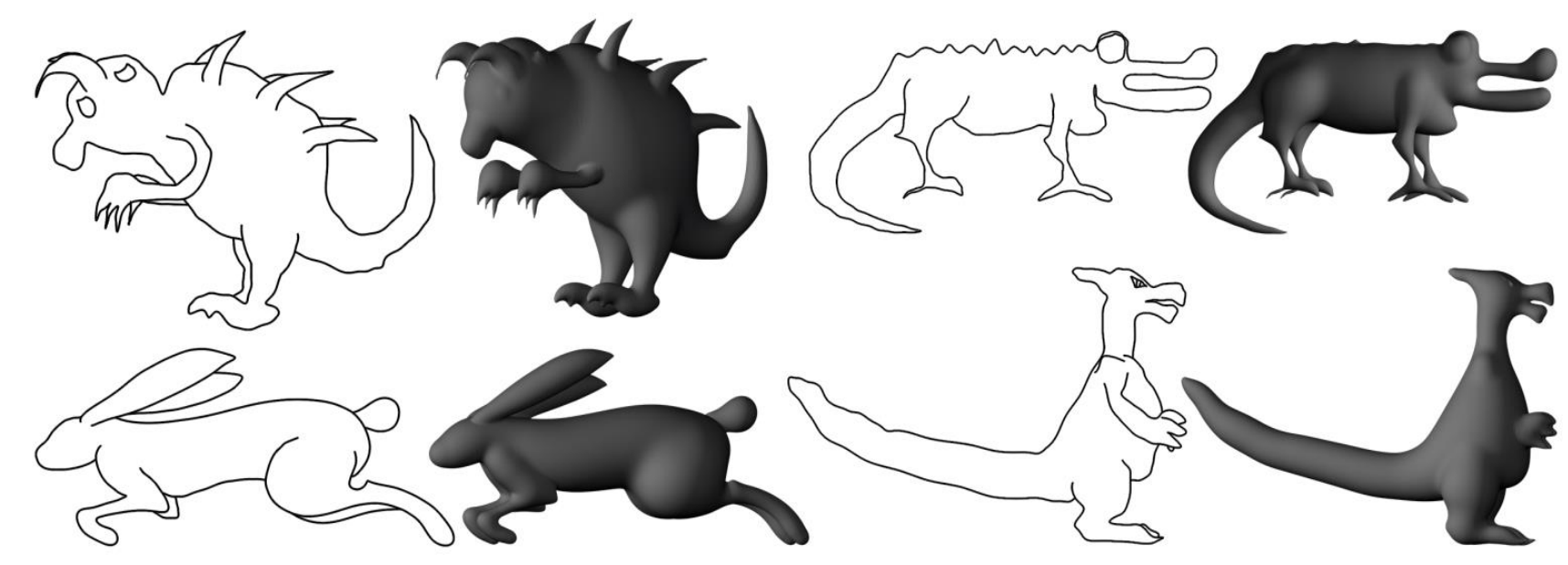




\section{Sketching specific shapes Example 2: Designing a tree}

\section{Challenges}

- Need to control a specific shape

- Too many branches for interactive modelling!

- Distributions matching laws from biology

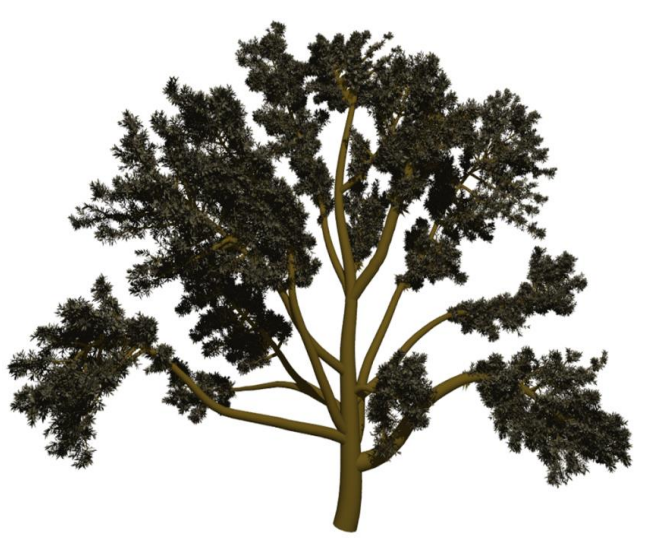

Inspiration

\section{Idea}
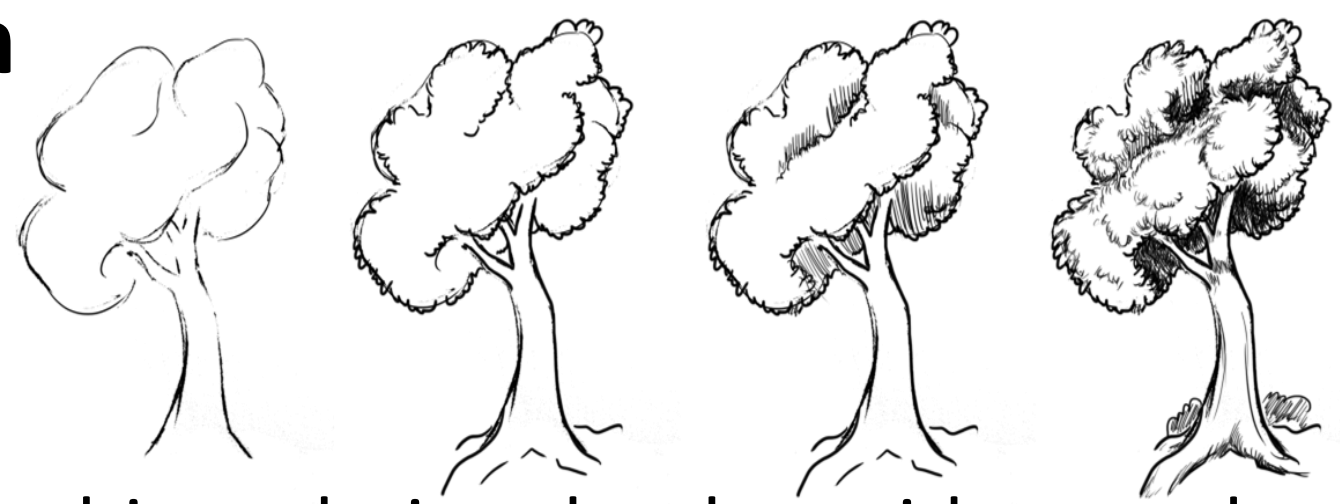

- Combine multi-resolution sketches with procedural generation! 


\section{Designing a tree}

- Structure from silhouette!

- Use rules from botanic and probabilit

- Infer sub-structures

- Adapt branching style

- Extend branches to 3D
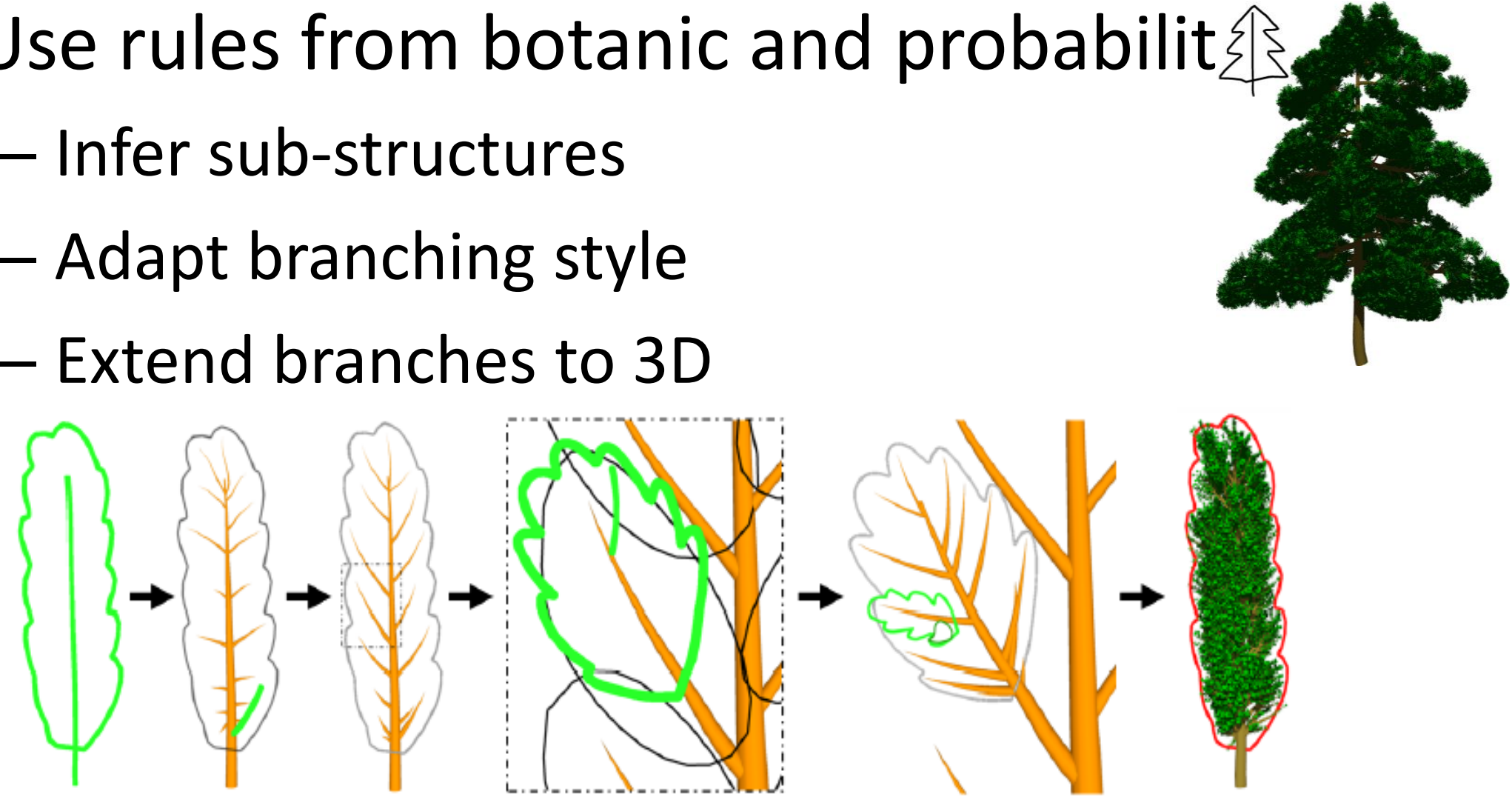

Q9. SIGGRAPH 


\section{Creating lots of elements Designing a tree}

- Silhouette analysis

- Medial axis?

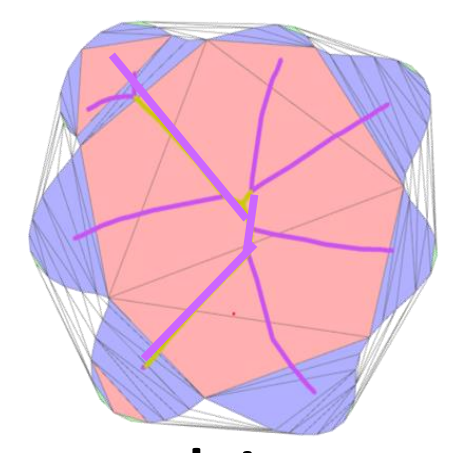

- Add knowledge on branching arrangements

- Find shortest branch
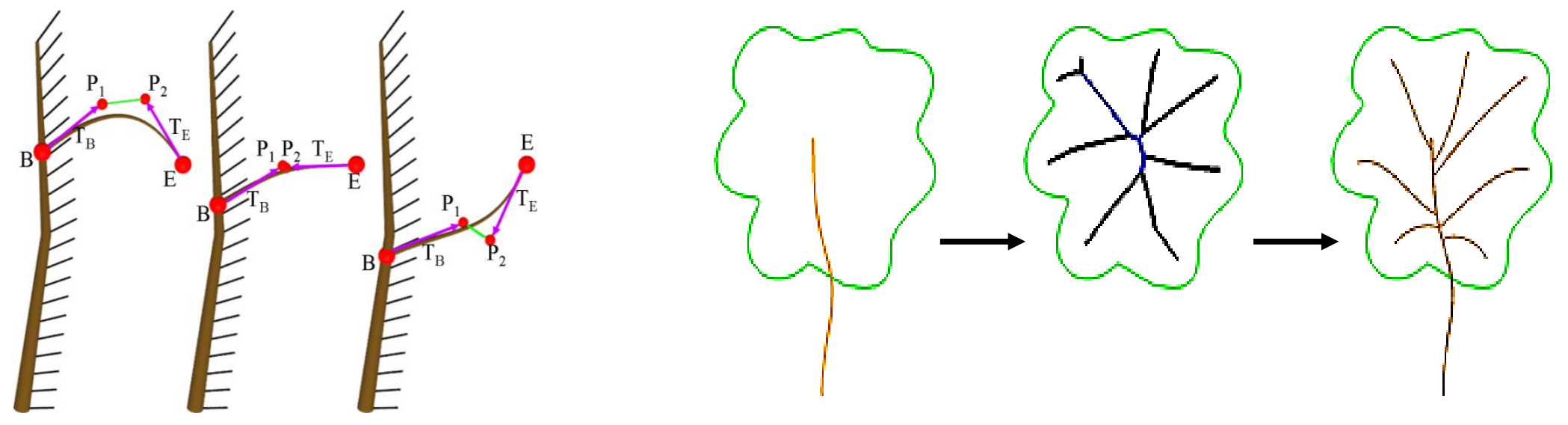


\section{Creating lots of elements Designing a tree}

- Inferring substructures
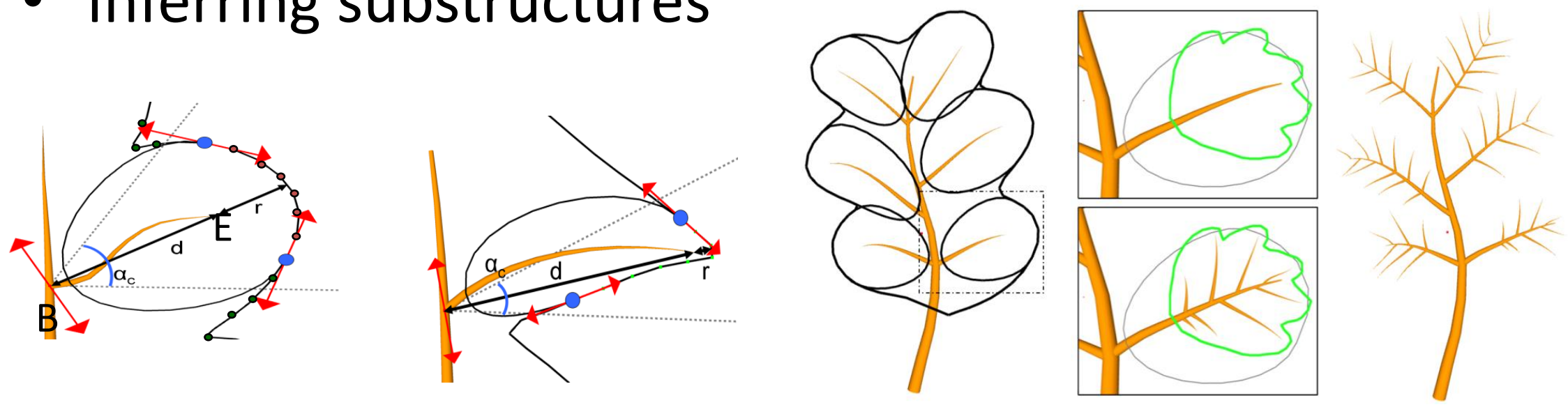

- Inferring 3D branch distributions
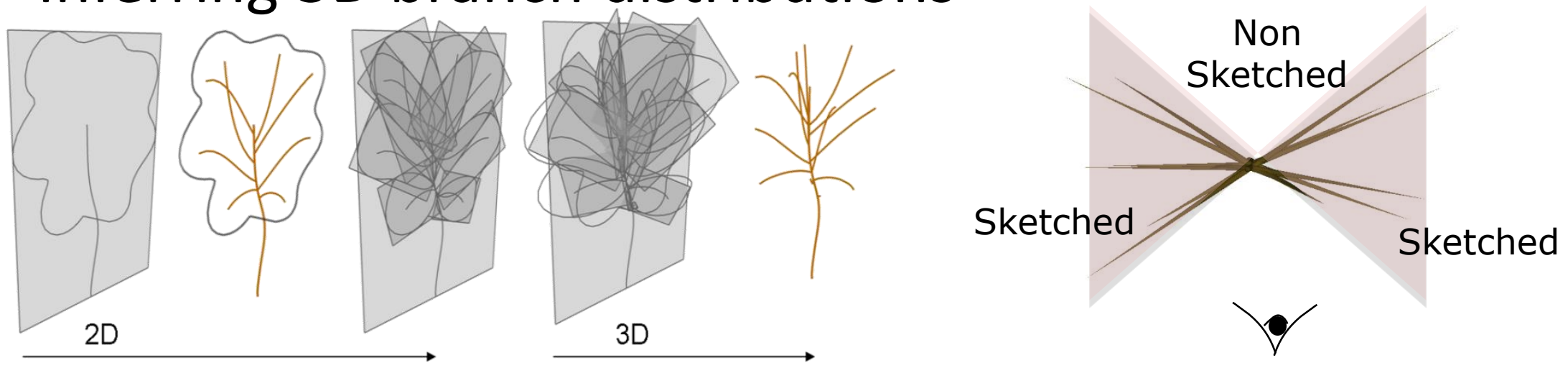

O? SIGGRAPH 


\section{Example 2 \\ Designing a tree}

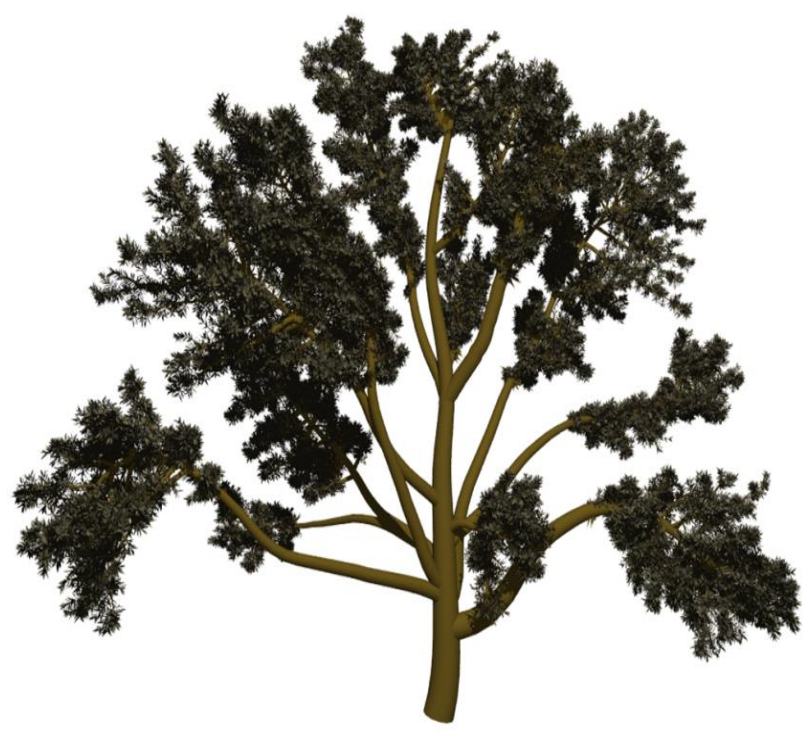

Eucalyptus tree

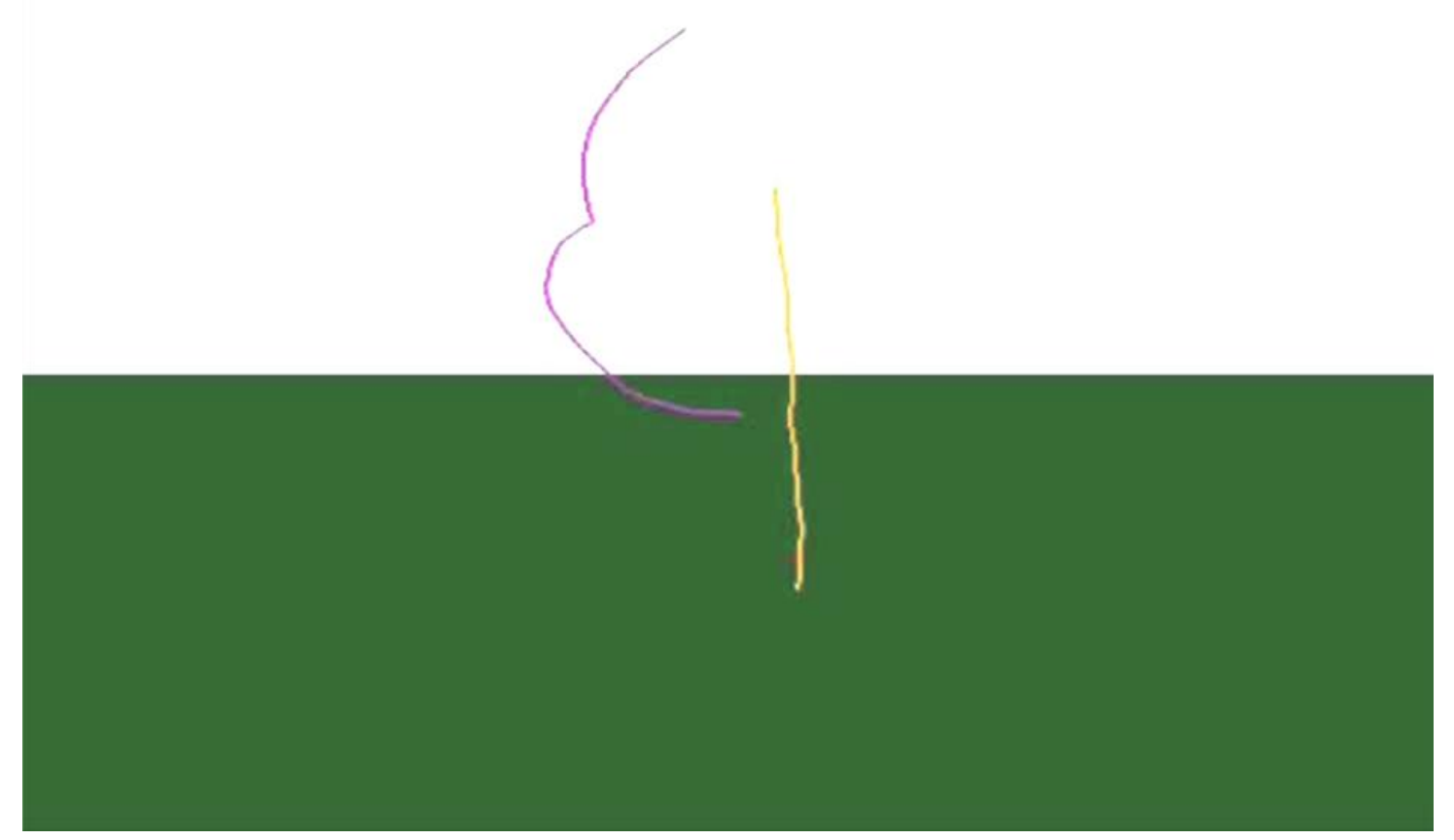

[Wither, Boudon, Cani, Godin, Eurographics 2009] 


\section{Sketching using prior knowledge Example 3: Clothing design}

Standard method to create garments

- Design \& place patterns

- Run a simulation

- Iterate!

Idea

- 3D garment from a single sketch?
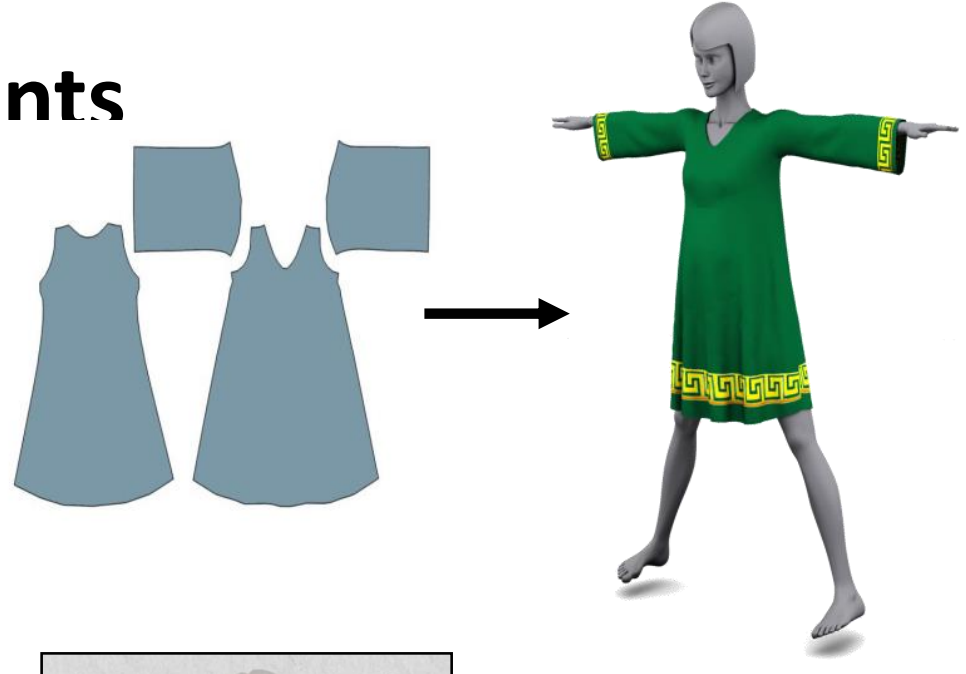

$\rightarrow$ automatic 3D + patterns!

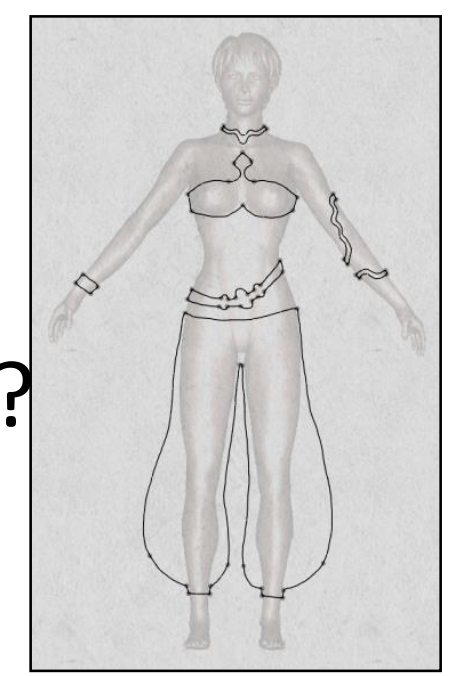




\section{Clothing design}

\section{Using silhouette information only}

\section{Virtual clothing from a sketch?}

- Sketch on a view of a 3D model

- Knowledge? Rule of thumb

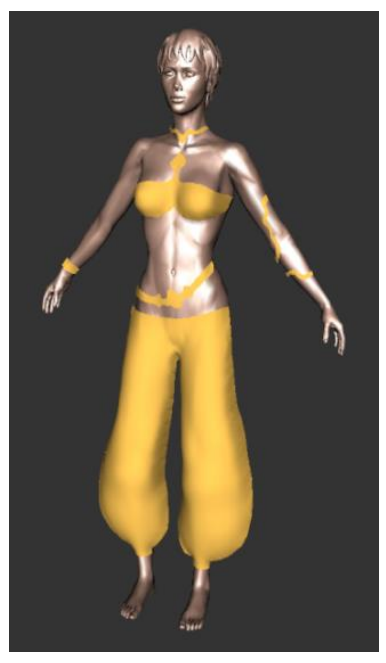

- Fitting is the same in all directions!
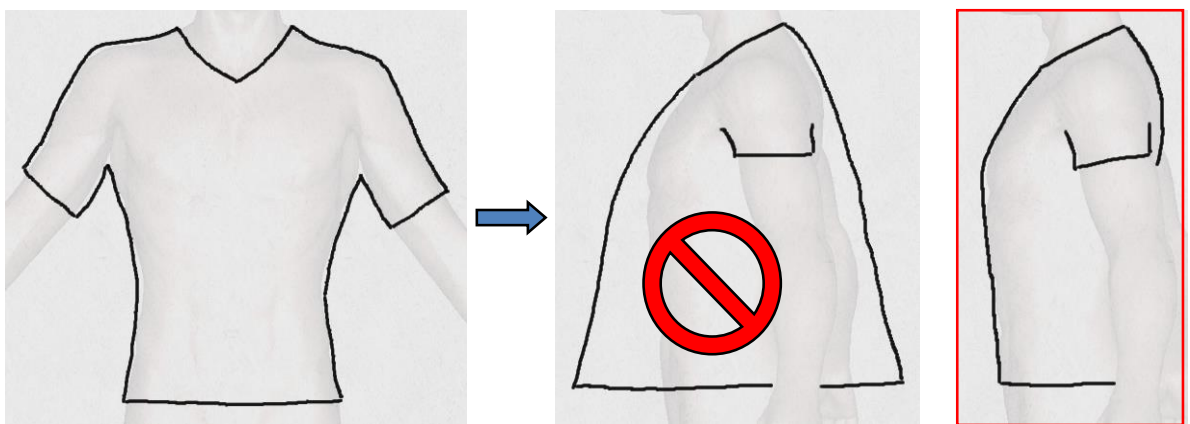

Sketch in a distance field! 


\section{Clothing design \\ Using silhouette information only}

Results lack folds!

- Allow the designer to sketch them?
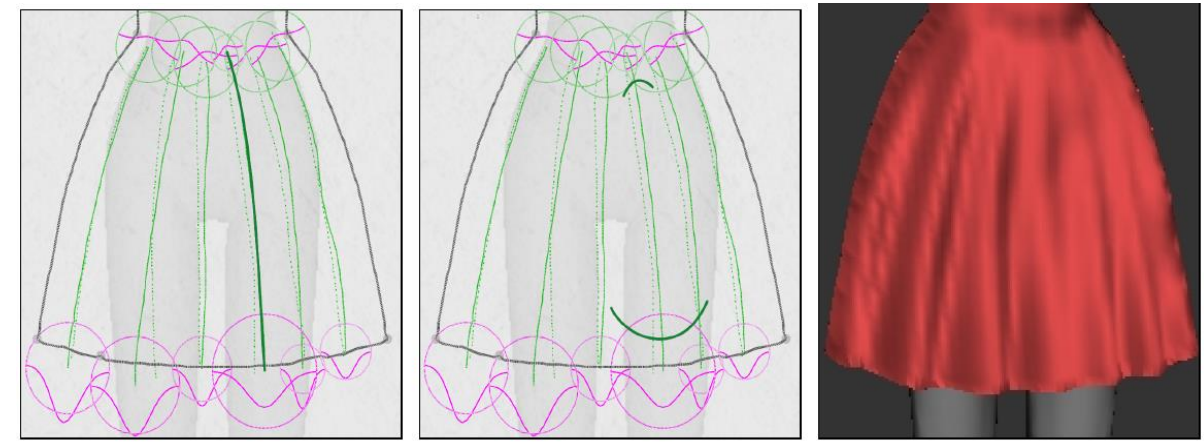

[Turquin, Cani, Hughes IEEE CG\&A 2007]

- Requires specific skills!

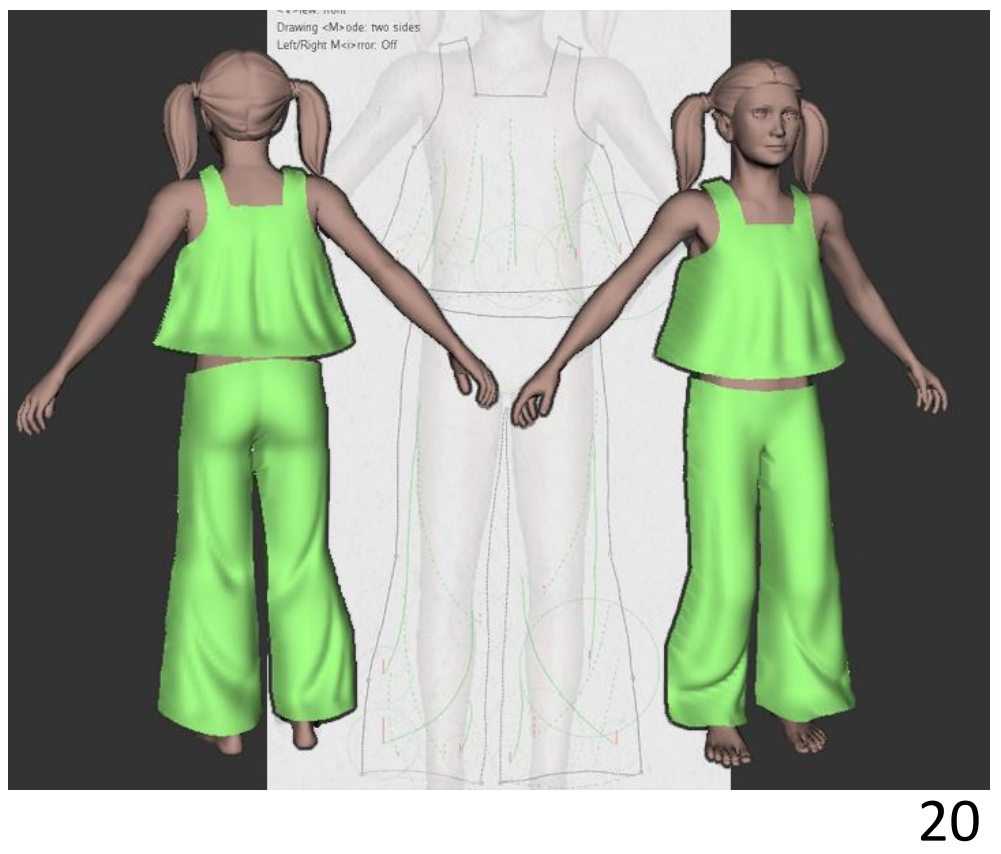

OP, SIGGRAPH

ASIA 2016

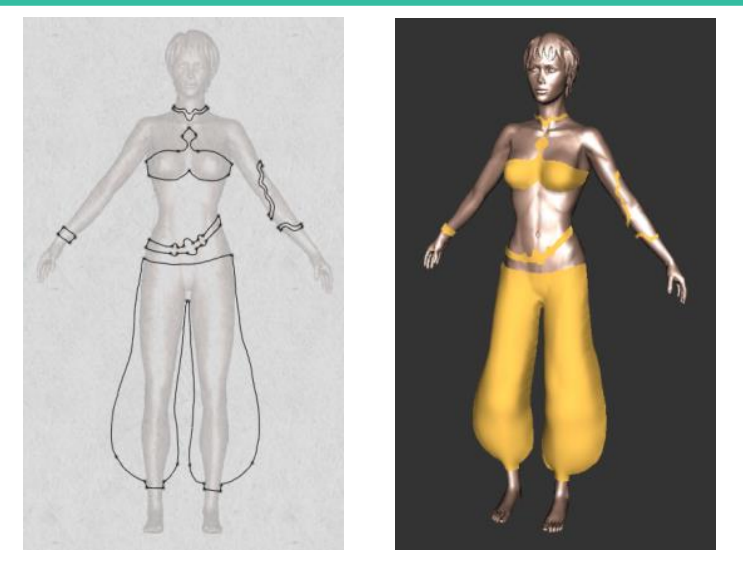




\section{Clothing design}

\section{Using silhouette information only}

Results lack folds!

- Use more a priori knowledge?

- Cloth is a developable material

- Folds can be simulated
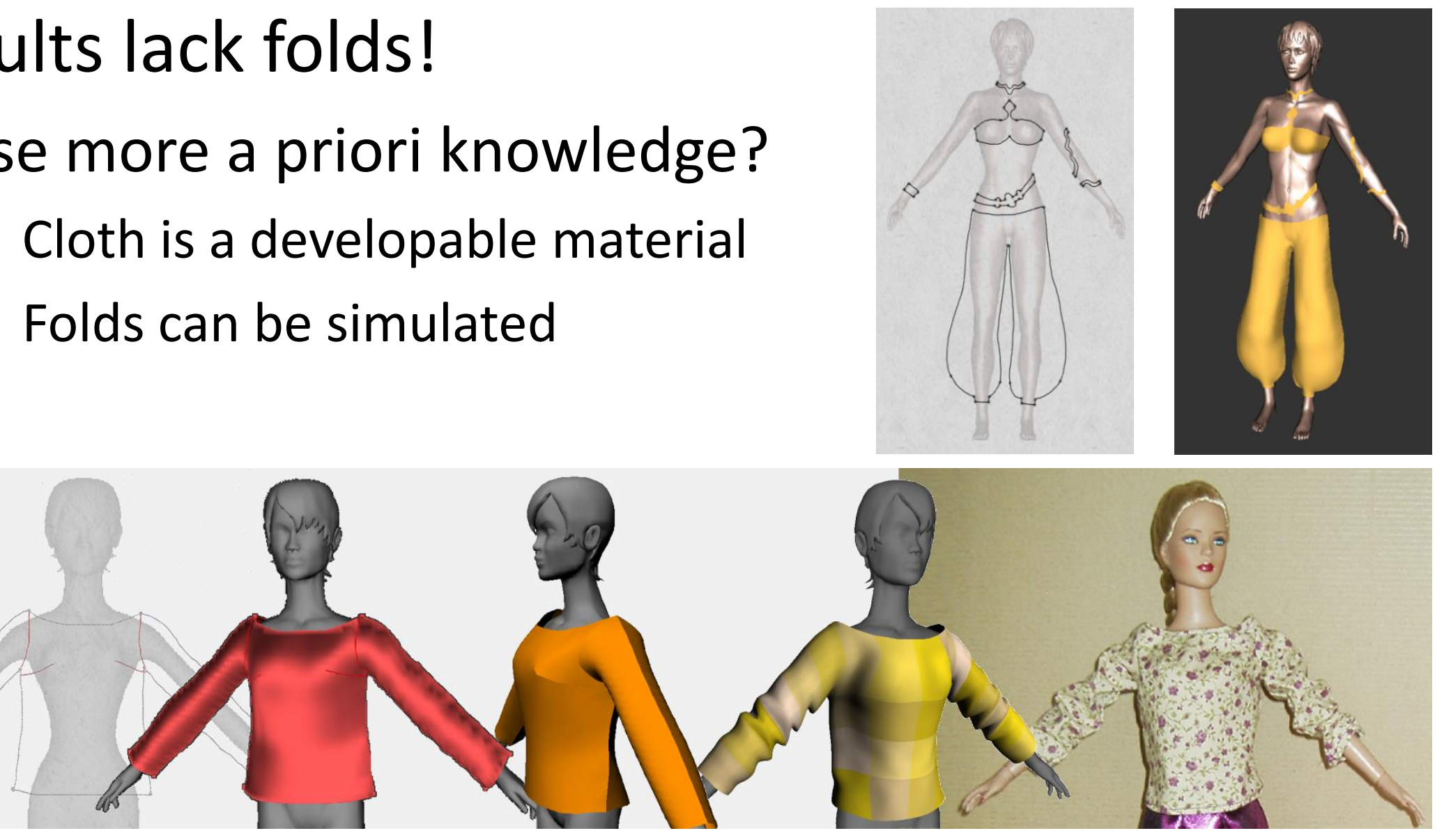

Q9. SIGGRAPH 


\section{Clothing design \\ Developable surfaces from a sketch}

Developable surface from sketch?

- Solution 1: increase developability

- Start with the rough surface

- Locally optimize the shape (1D normal mal

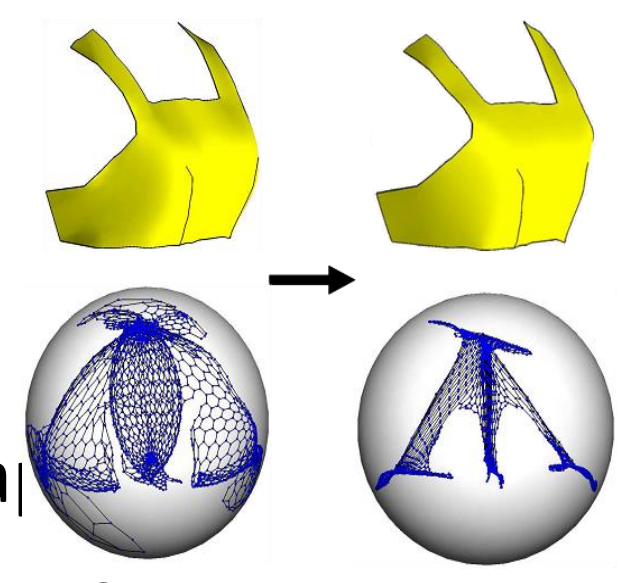

- Solution 2: smooth developable surface from contours

Convex edges?

Recursively split $\&$ triangulate the convex hull
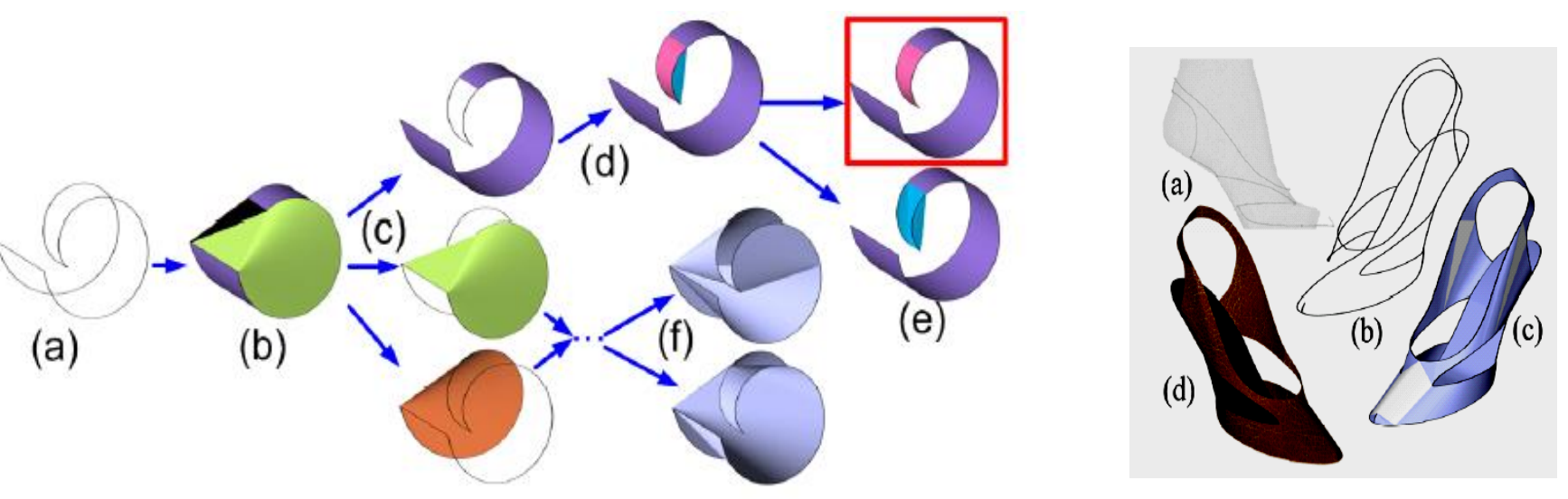


\section{Clothing design}

\section{Developable surfaces from a sketch}
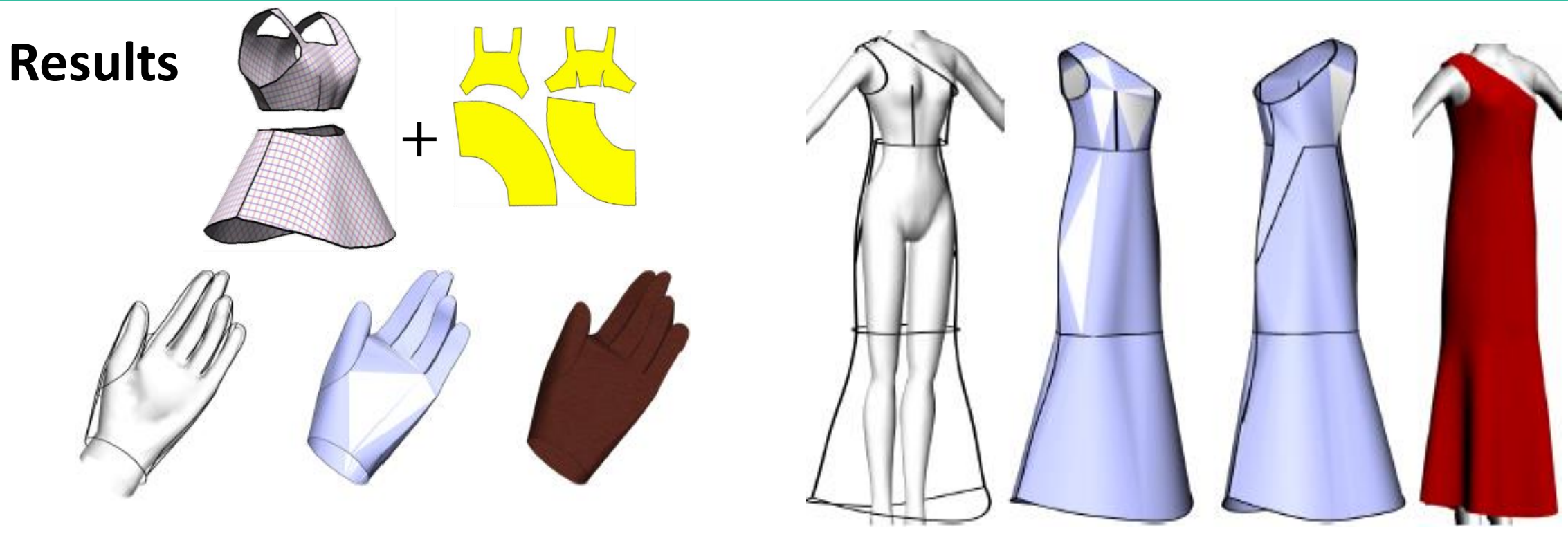

Folds from simulation

- Physically-based parameters required

- Stiff system for un-extensible cloth

- Ideal fold-free surface to be drawn at the design stage! 


\section{Clothing design Sketching folds?}

Folds may be part of design!
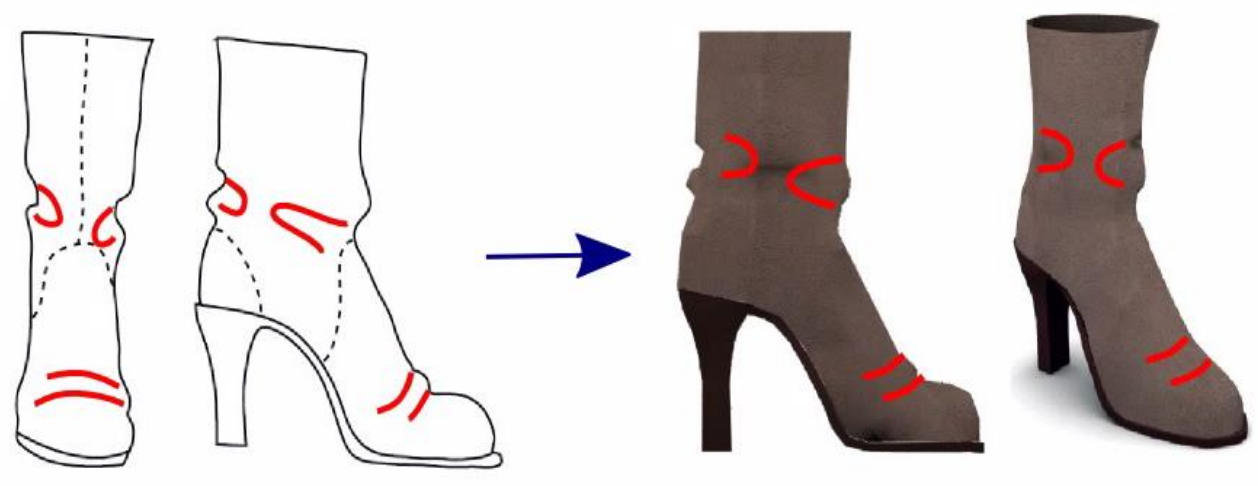

Challenge: Handling non-flat silhouettes !
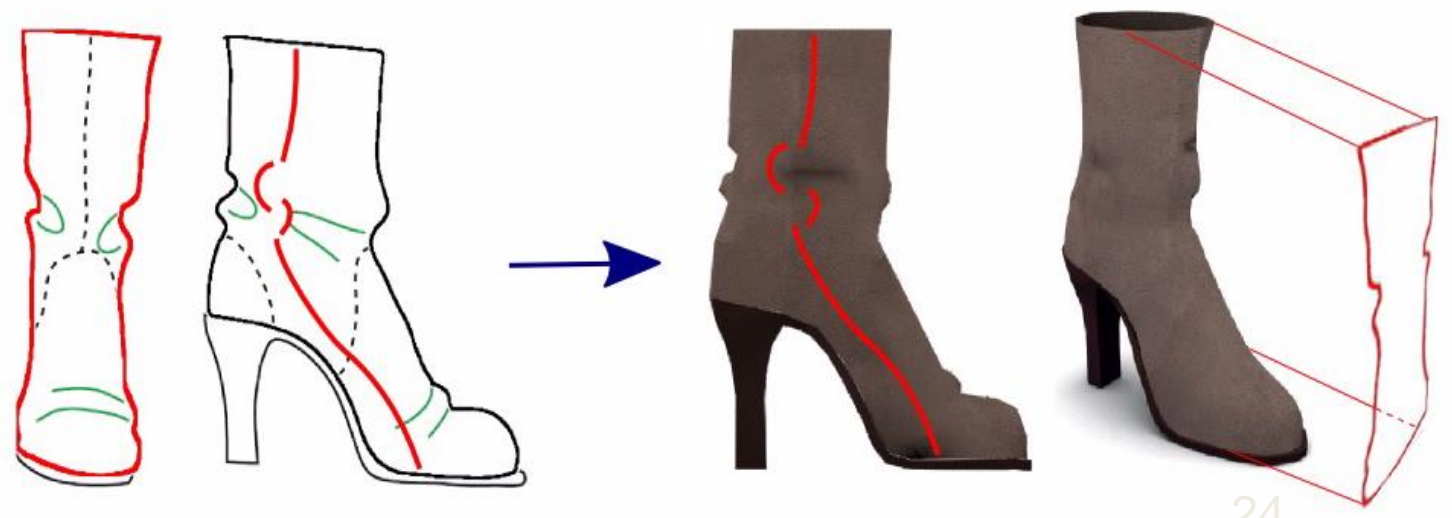

ASIA 2516 


\section{Clothing design}

Sketching a folded surface

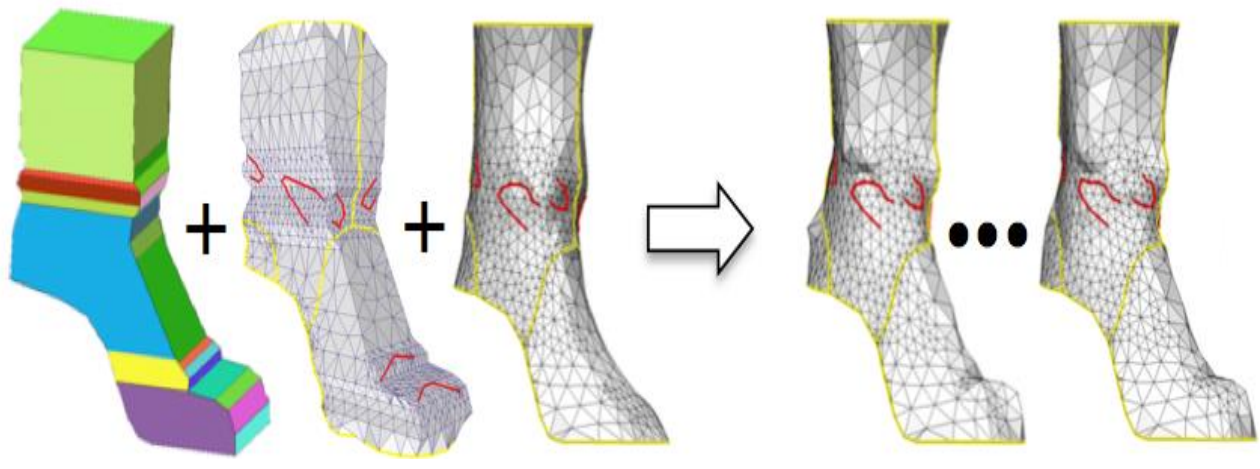

Iterate

- Optimize developability

- Match the sketch
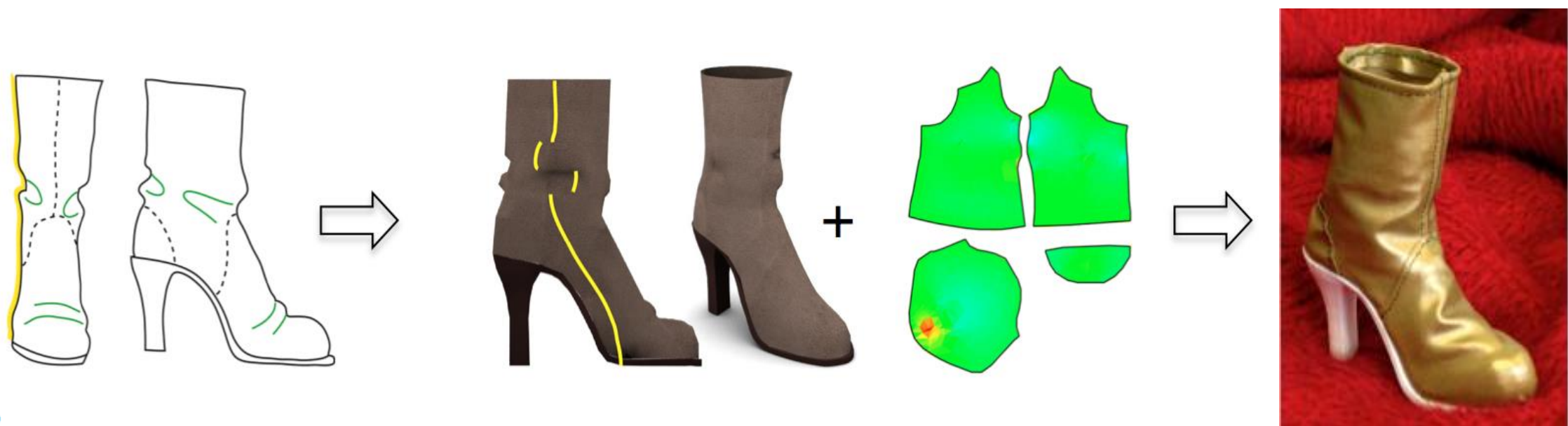

QQ. SIGGRAPH

ASIA 2016 


\section{Initialization}

Visual hull from silhouettes

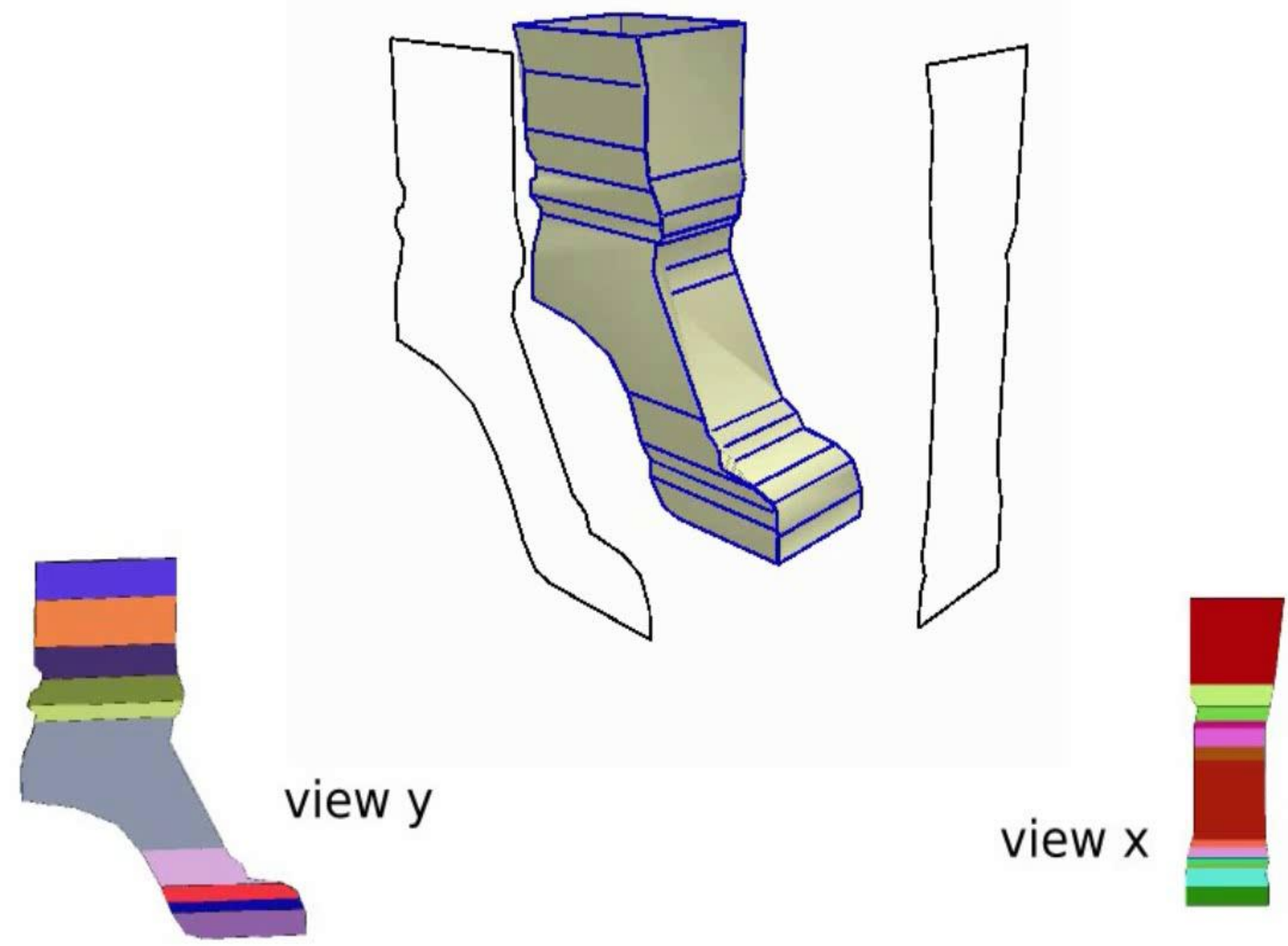




\section{Clothing design \\ Sketching a folded surface: Results}
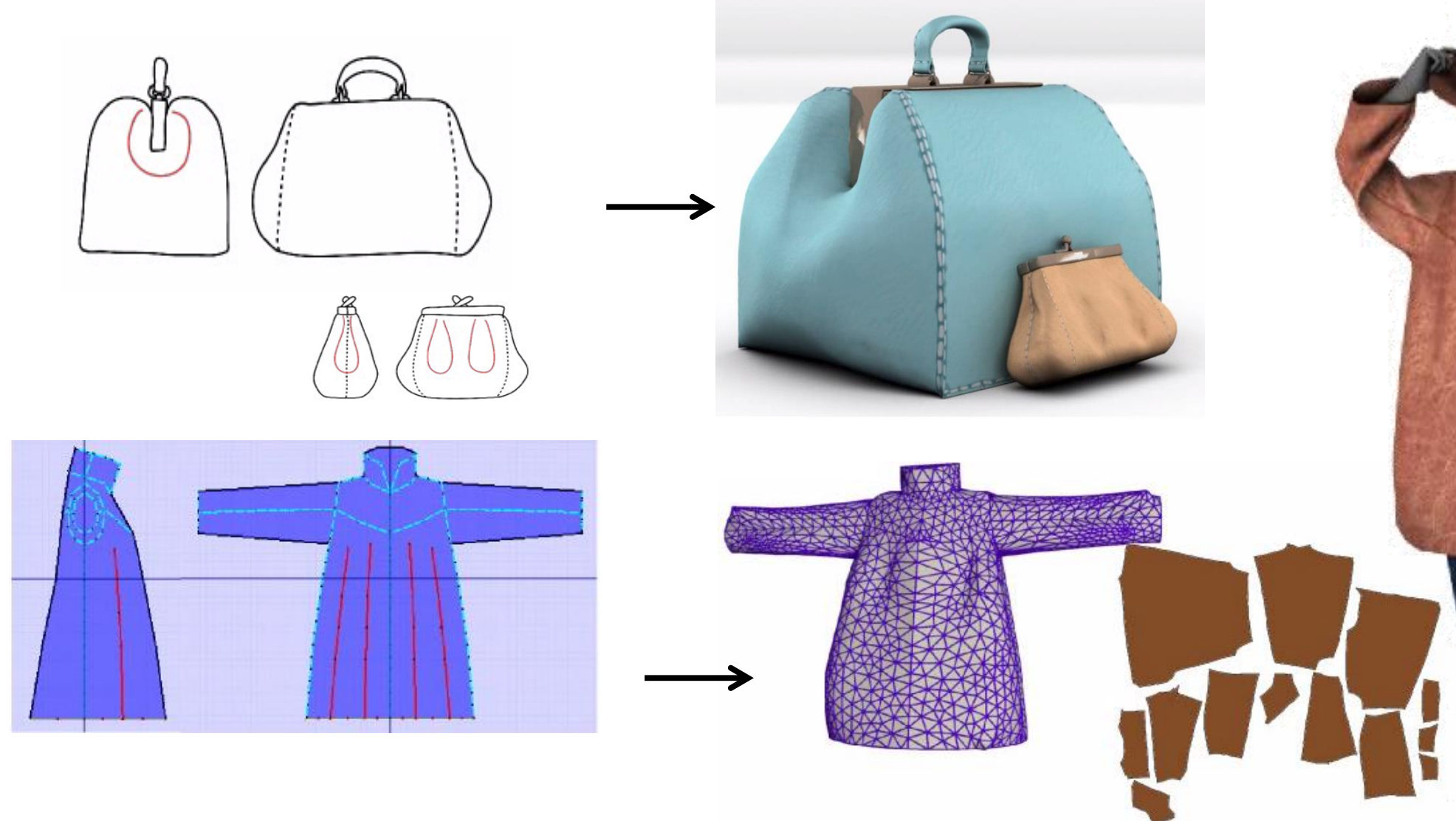

[Jung, Hahmann, Rohmer, Bégault, Boissieux, Cani, ACM TOG 2015]

Q Q SIGGRAPH

- ASIA 2016 


\section{Conclusion}

\section{Sketch-based modelling using prior knowledge}

+ Knowledge helps filling in missing data

+ High quality modeling (constraints met)

$\rightarrow$ Fast 3D from a single sketch!

- A system for each application...

Next: more general methods!

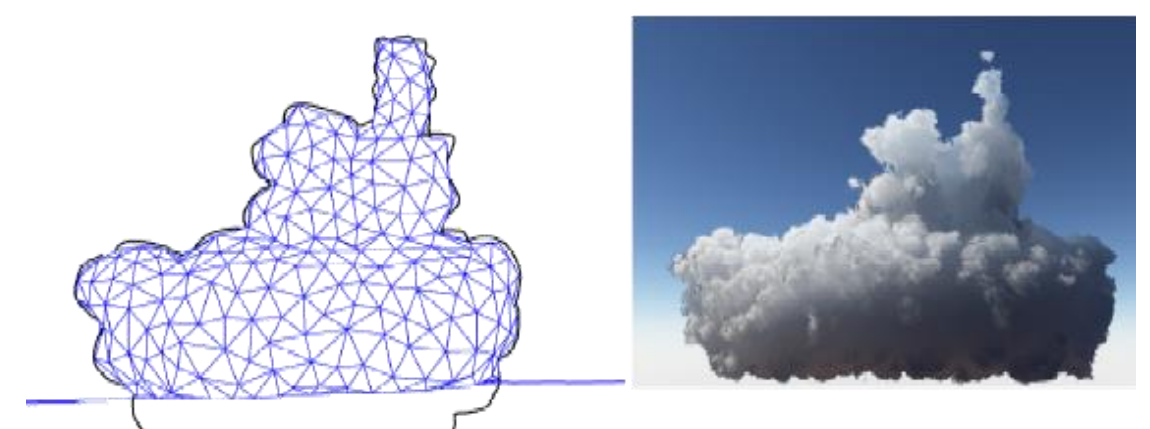

[Wither Bouthors Cani 2008]

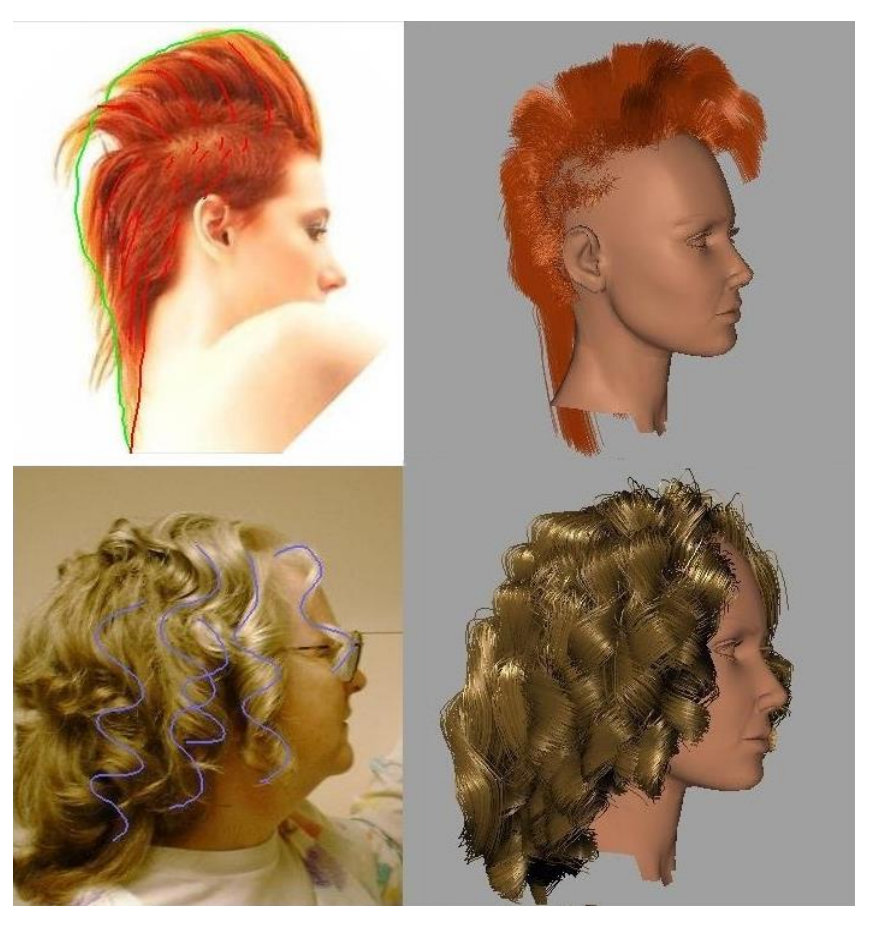

[Wither Bertails Cani 2007] 


\section{References}

- Bernhardt, Pihuit, CANI, Barthe - Matisse: Painting 2D regions for modeling freeform shapes, In SBIM 2008.

- ZanNI, Bernhardt, Quiblier, CANI - Scale invariant integral surfaces. Computer Graphics Forum 32(8), 2013.

- Entem, Barthe, Cani - Modeling 3D animals from a side-view sketch . In SMI 2014.

- Wither, Boudon, CANI, Godin, Computer Graphics Forum 28(2), Eurographics 2009.

- TURQUin, Wither, BoISSIEUX, CANI, HUGHES - A sketching interface for clothing virtual characters. IEEE Computer Graphics and Applications, 27 (1), 2007.

- DeCaudin, Julius, Wither, BoIssieuX, ShefFer, CANI. - Virtual Garments: A Fully Geometric Approach for Clothing, Computer Graphics Forum, Eurographics, 2006

- ROSE ET AL. - Developable surfaces from arbitrary sketched boundaries, SGP 2007.

- Jung, hahmann, Rohmer, Begault, Boissieux, Cani - Sketching Folds, ACM TOG 2015.

- Wither, Bouthors, CaNI - Rapid sketch modeling of clouds, In SBIM 2008.

- Wither, BerTaILS, CANI - Realistic Hair from a Sketch, In SMI, 2007. 
Thanks to my students and collaborators!

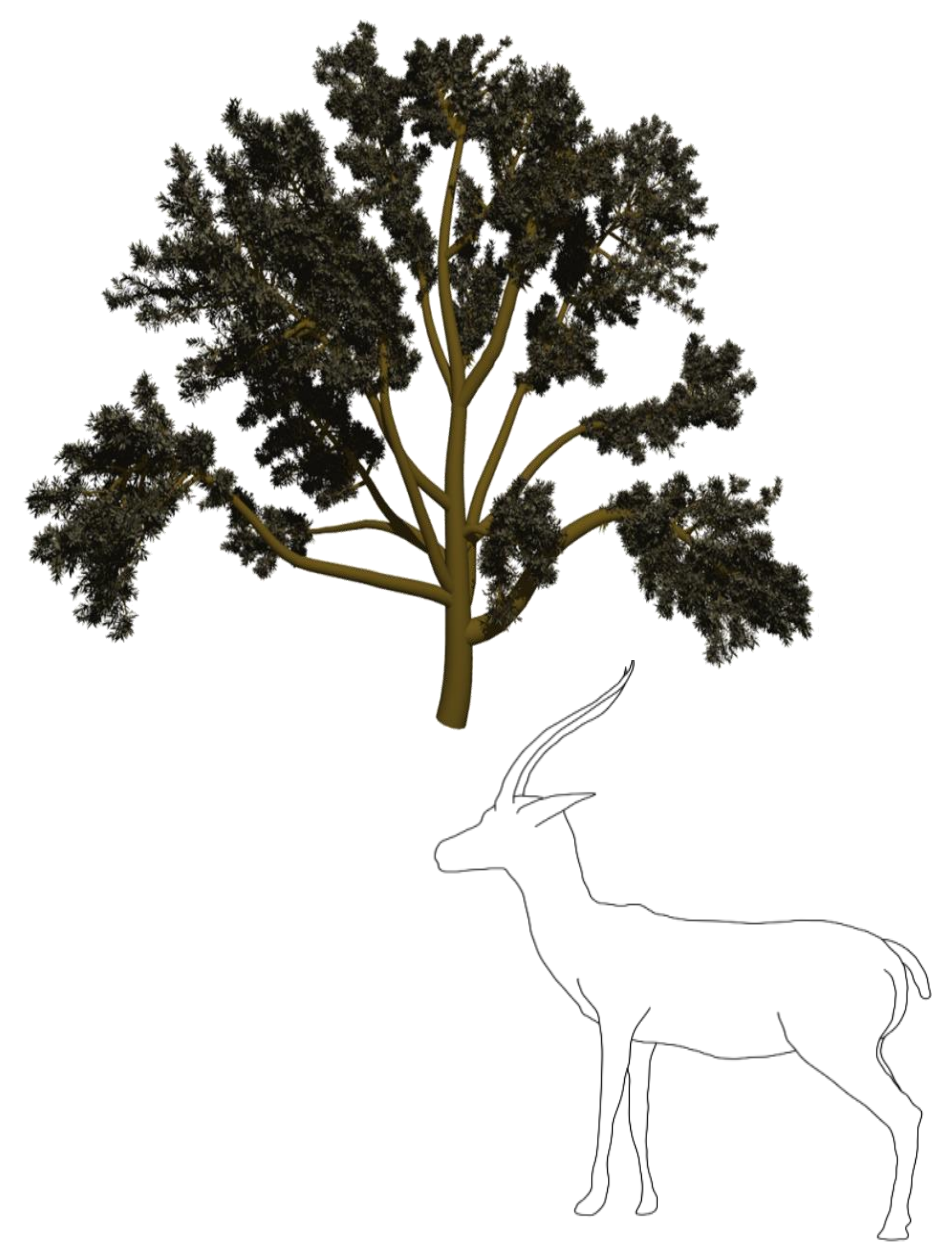

Q19. SIGGRAPH

ASIA 2916
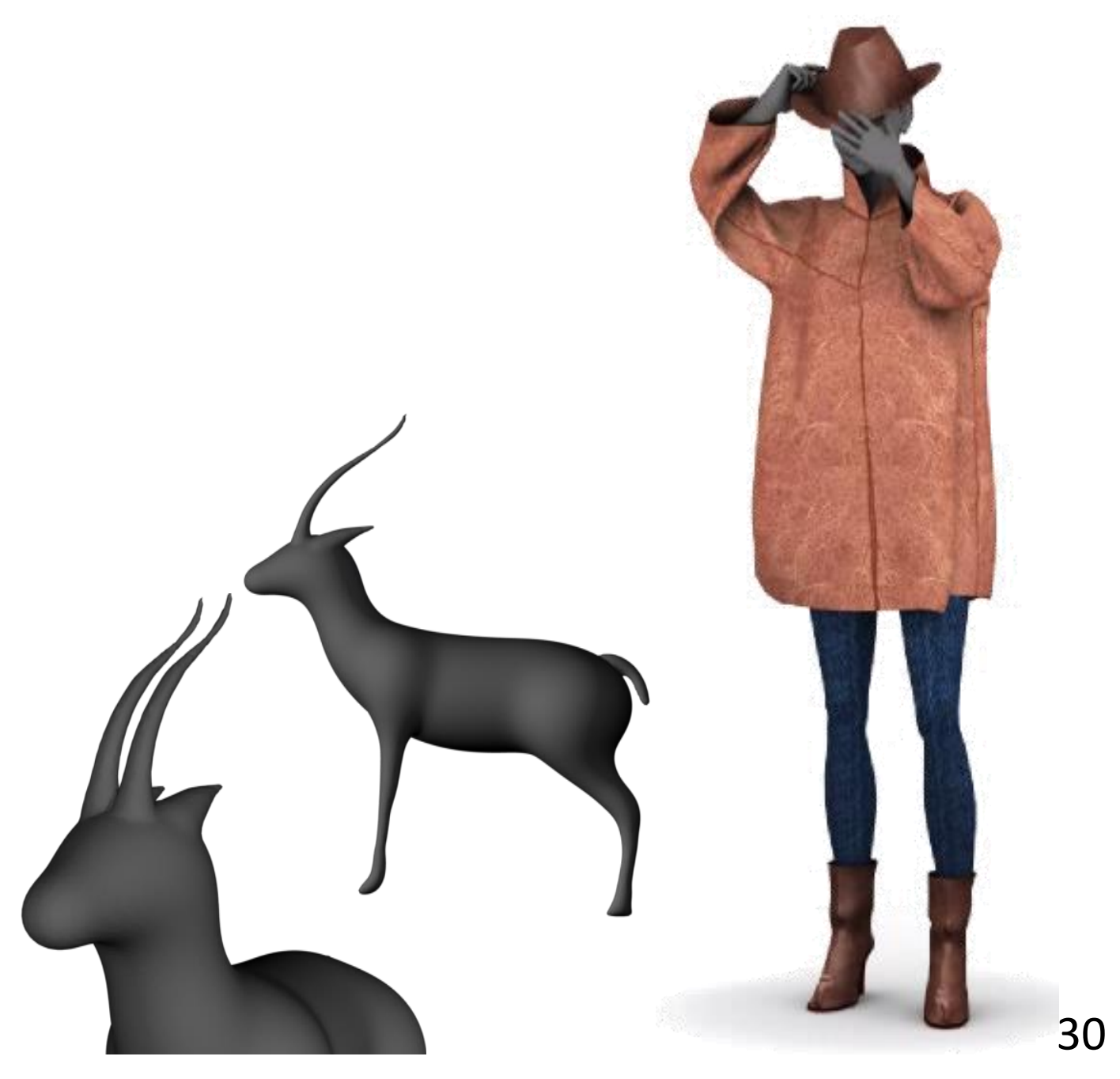
Single-view sketch-based modeling of 3D curves and surfaces

\section{Part I}

Yotam Gingold 


$$
a_{0}^{2}
$$




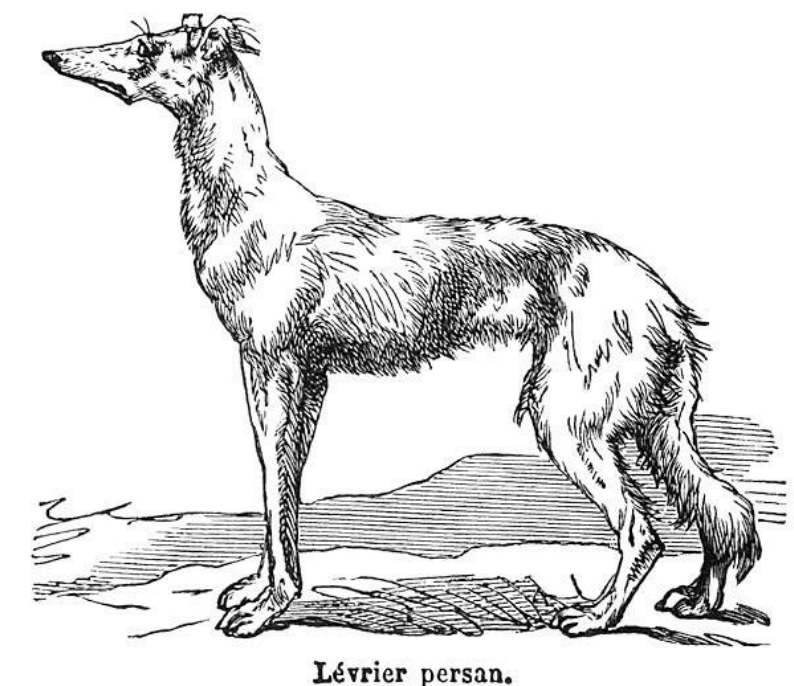

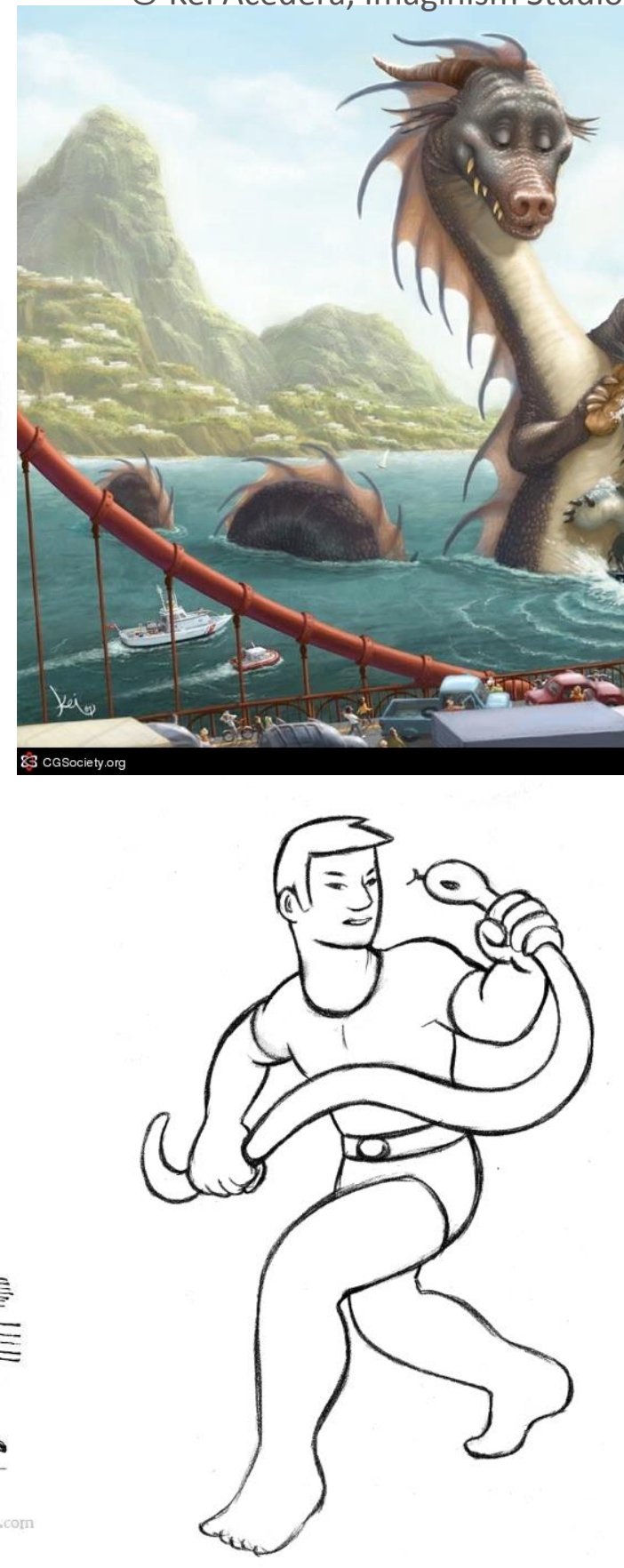

(C) Alex Rosmarin

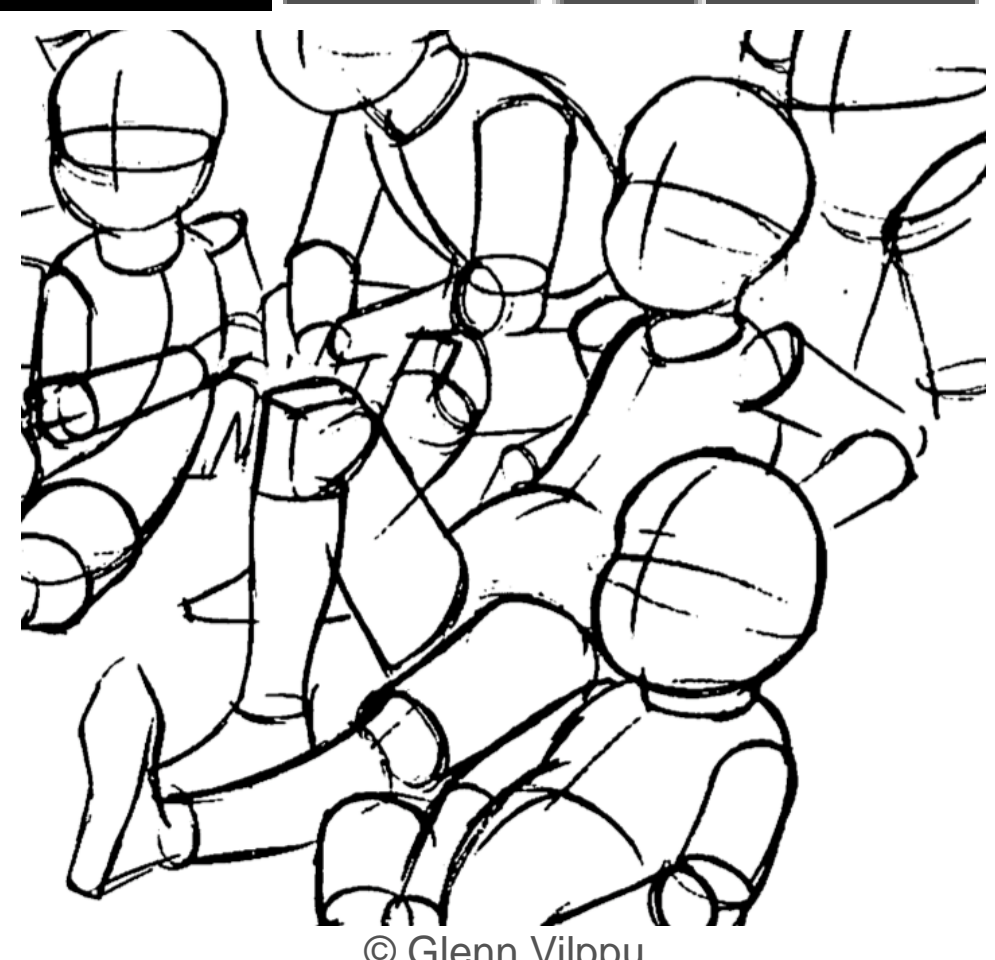




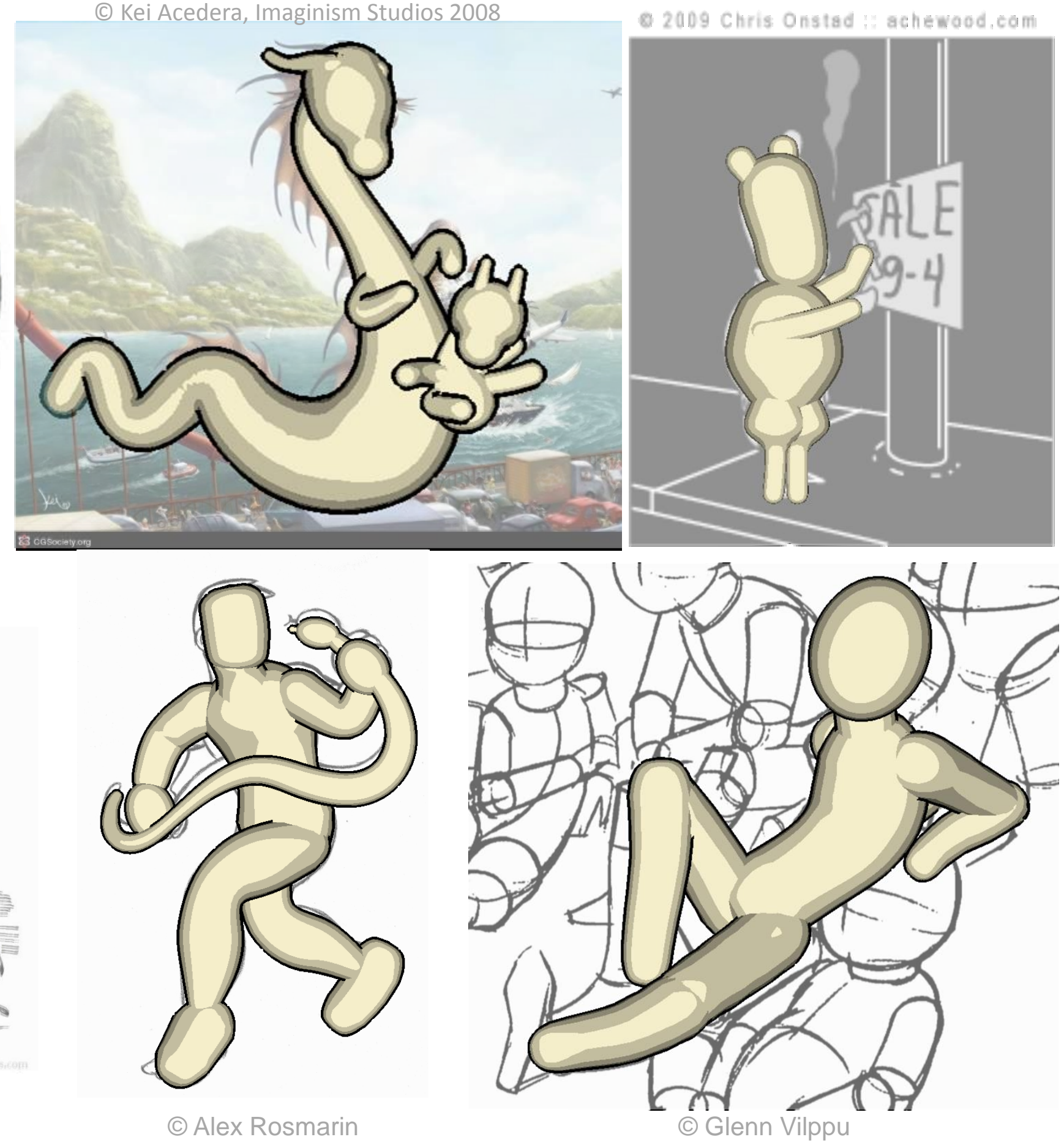




\section{Goals}

- Model by "describing" an existing 2D image with primitives and annotations.

- Usable by novices, including those with poor drawing skills.

- Create structured models. 
Sketch-based modeling with few strokes [Cherlin et al. 2005]

- Generalized cylinders with varying cross sections and "spines"
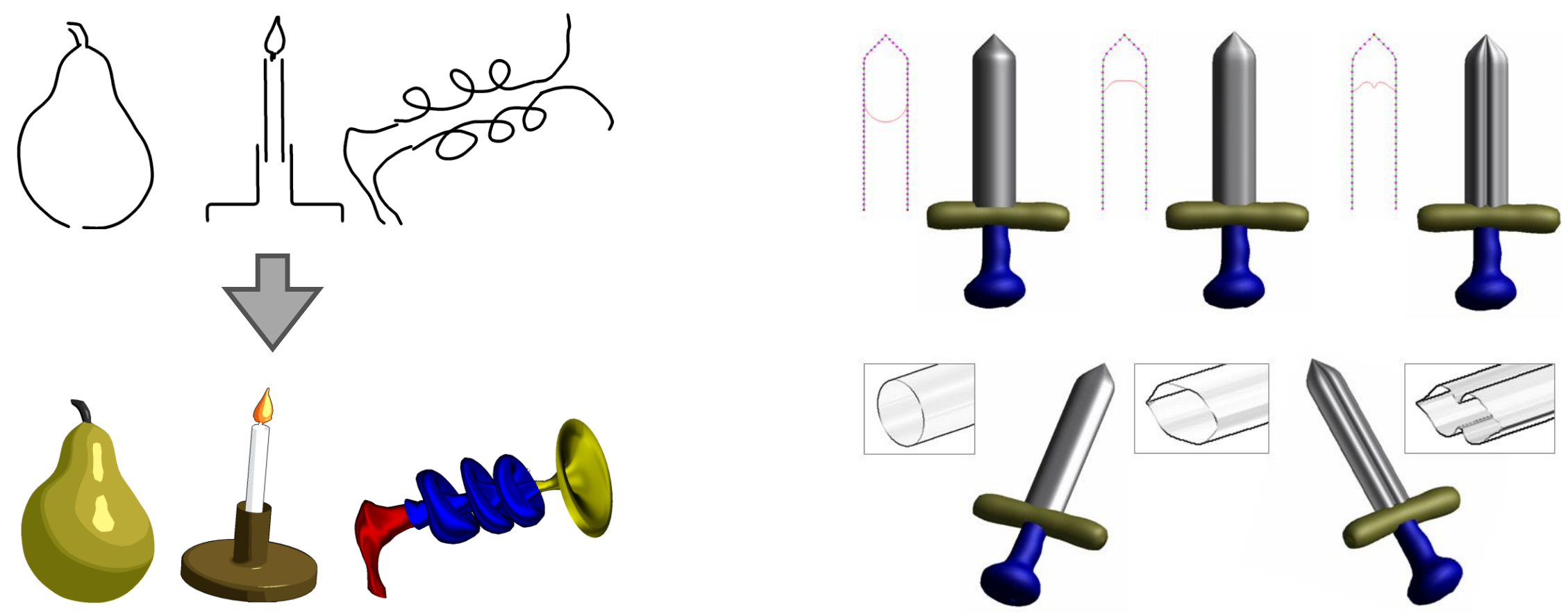

Q9, SIGGRAPH 


\section{Inspiration}

\section{D Drawing Approaches}
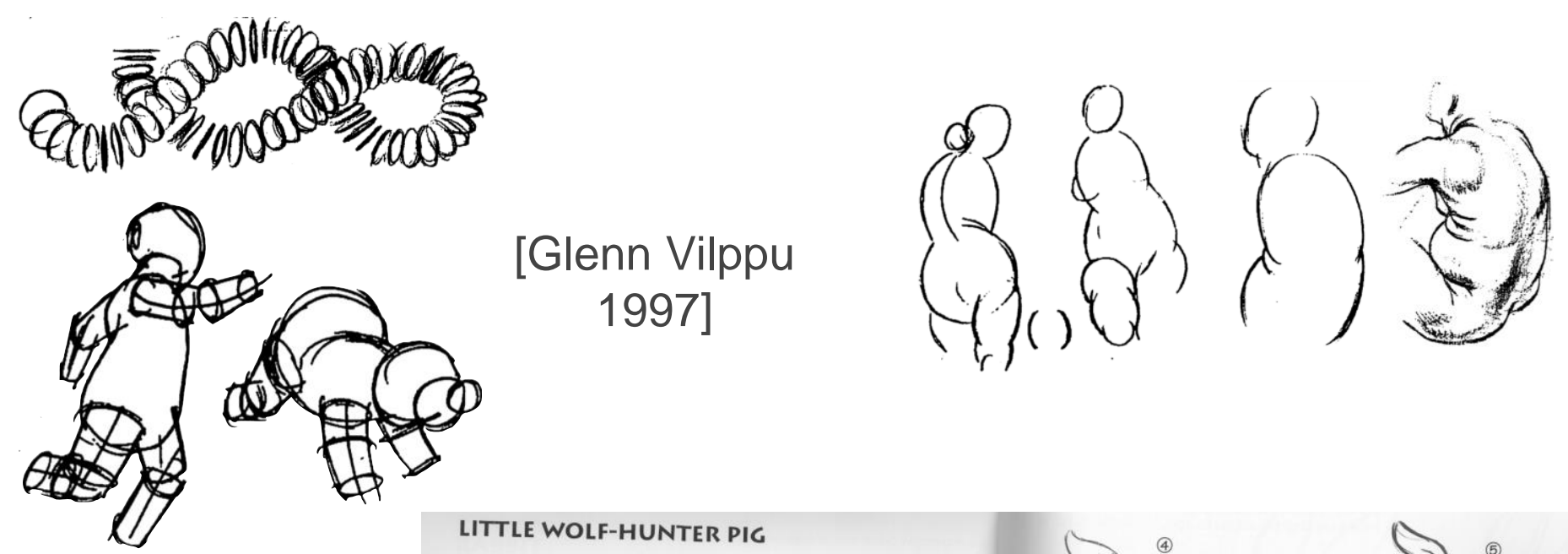

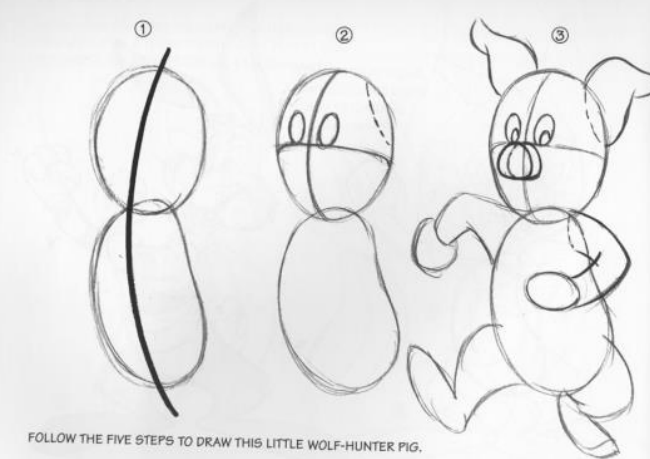

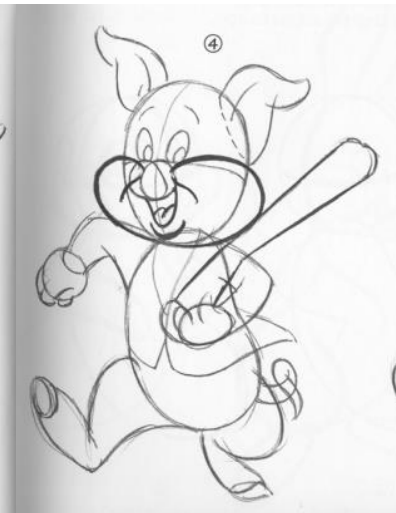

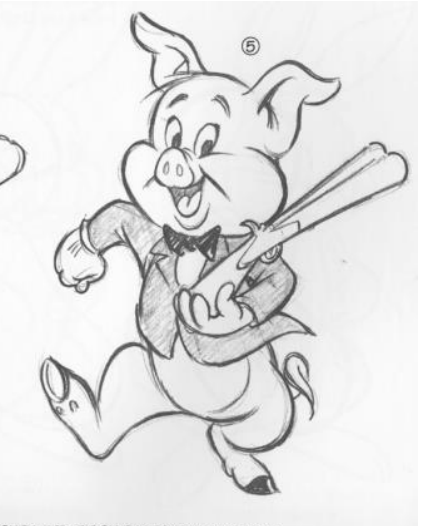

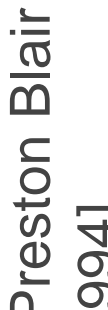




\section{Primitives}

\section{Generalized Cylinders \& Ellipsoids}

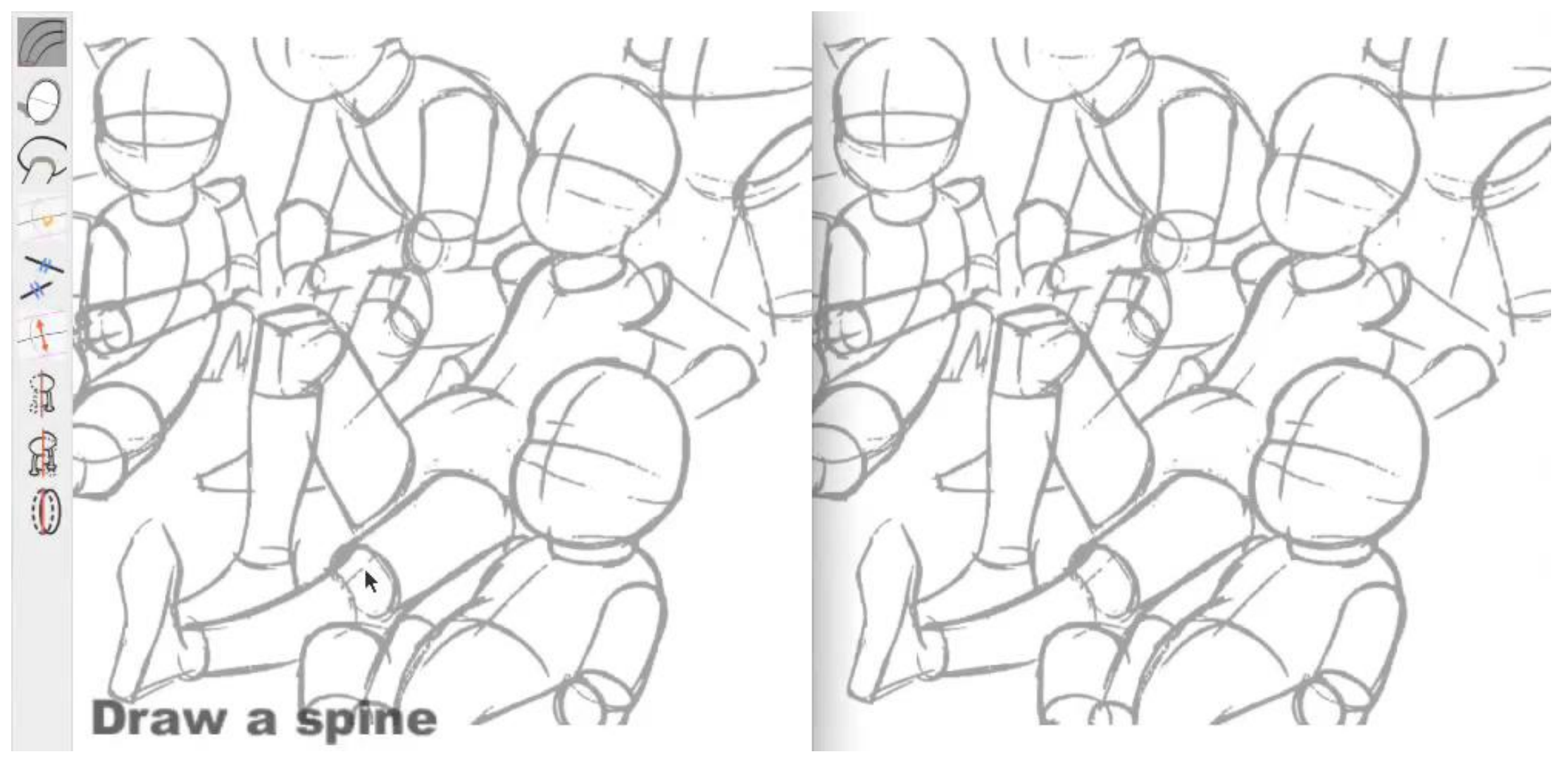

Q? SIGGRAPH

ASIA 2016

Guide image (C) Glenn Vilppu 


\section{Primitives}

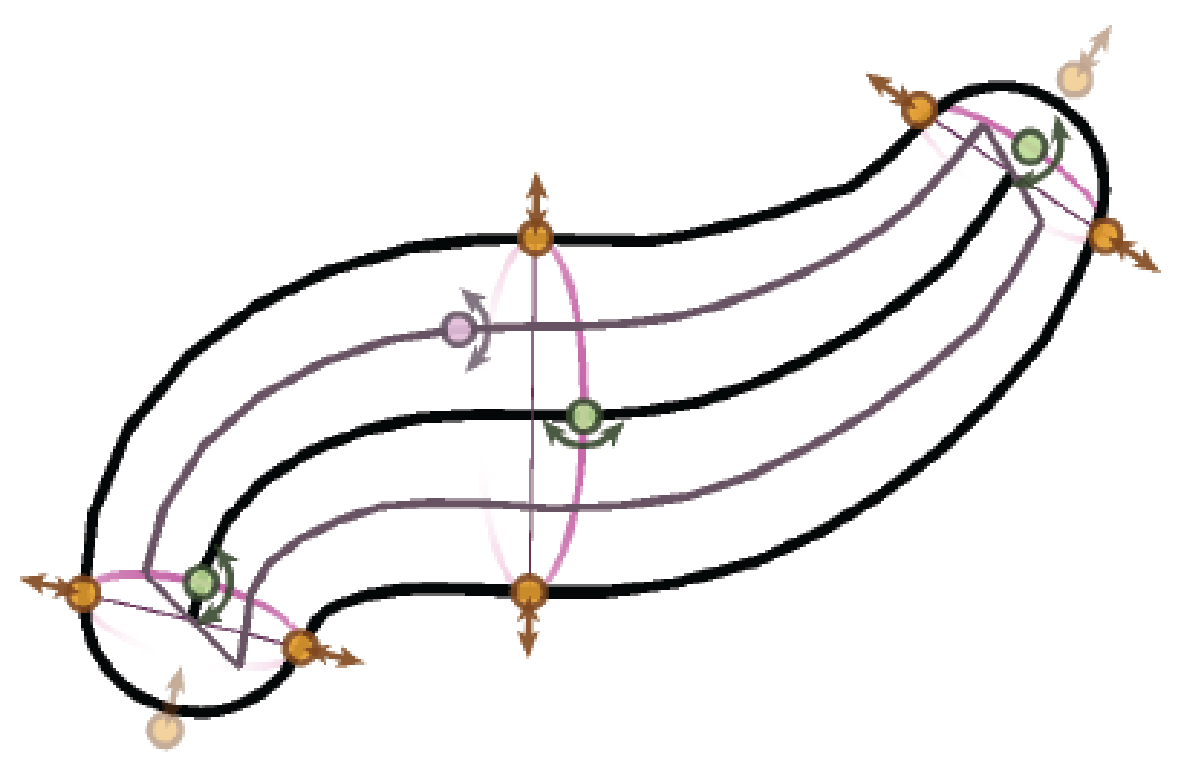

Generalized Cylinder

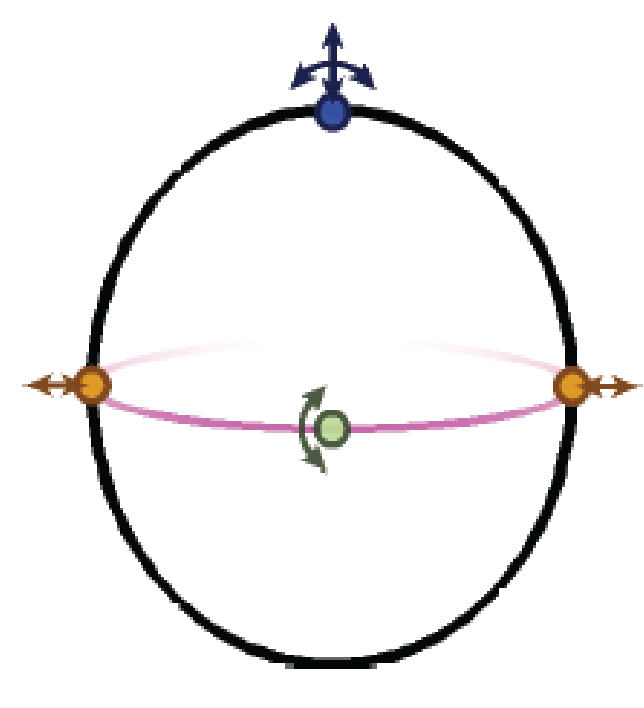

Ellipsoid 


\section{Primitive: Generalized Cylinder}

Generalized Cylinder 


\section{Annotations}

Same-length

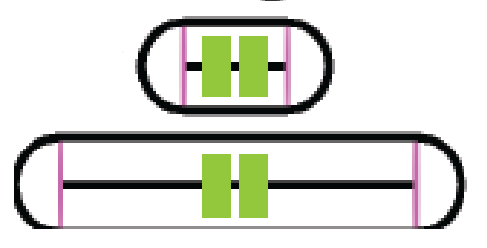

Same-tilt

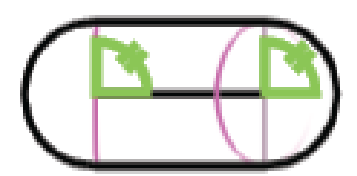

Same-scale

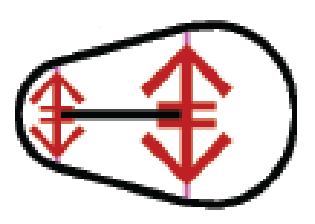

Connection curve

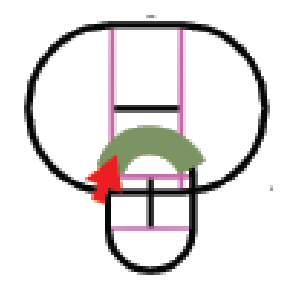

Mirror

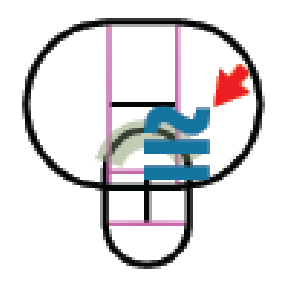

Alignment

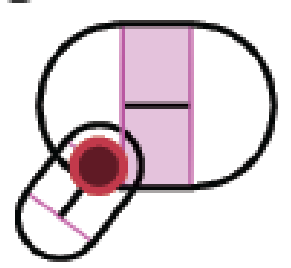

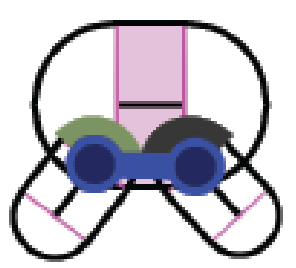




\section{Demo}

\section{Modeling Session 5x Speed}




\section{Results}

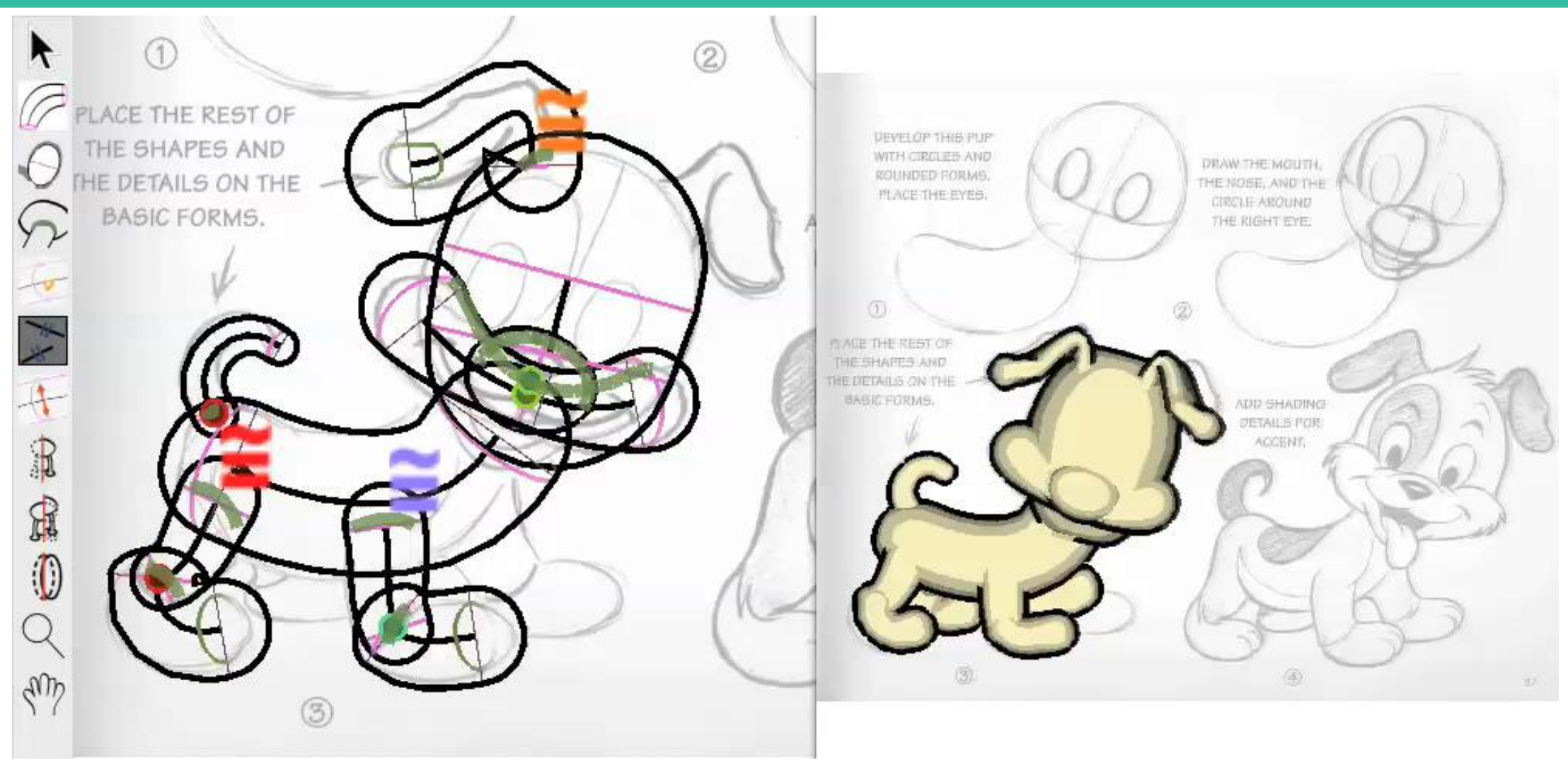

Guide images: (๑) Preston Blair; @ Alex Rosmarin; ( Kei Acedera, Imaginism Studios 2008; (

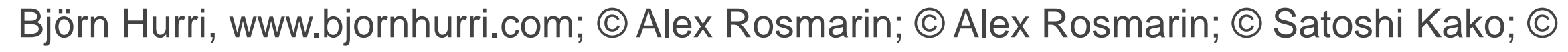
Satoshi Kako 


\section{Limitations}

Limited range of models

Can't be used for certain drawings

Doesn't actually use the guide image
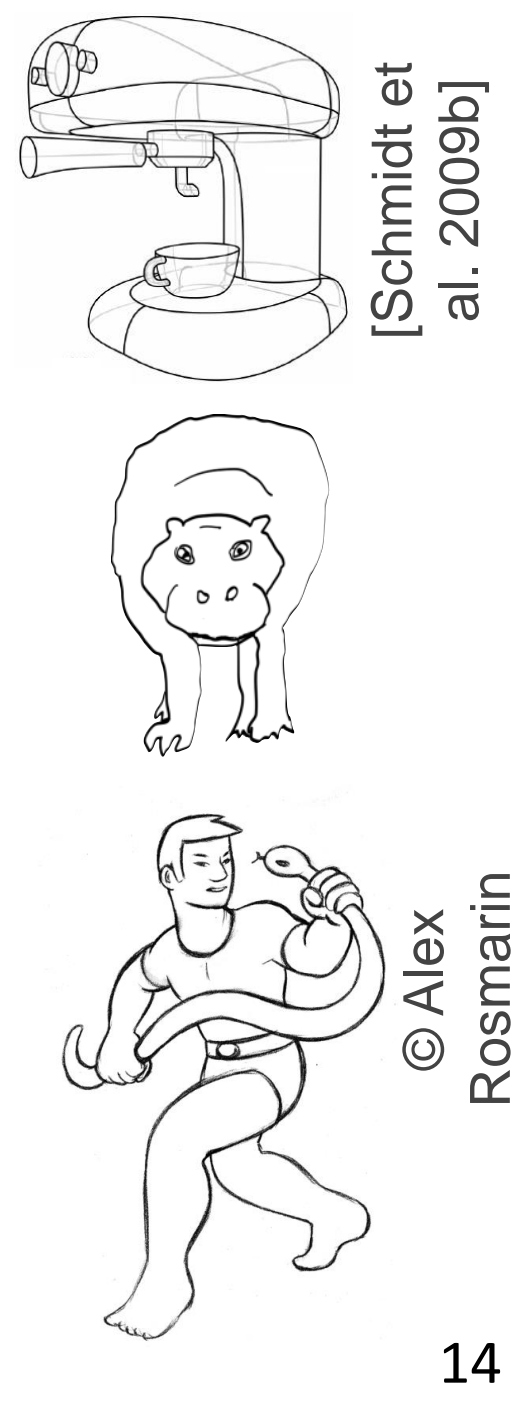


\section{Single-View Sketch-Based Modeling [Andre and Saito 2011]}

- Two perpendicular cross sections form the projection of a cubic corner (which is welldefined)

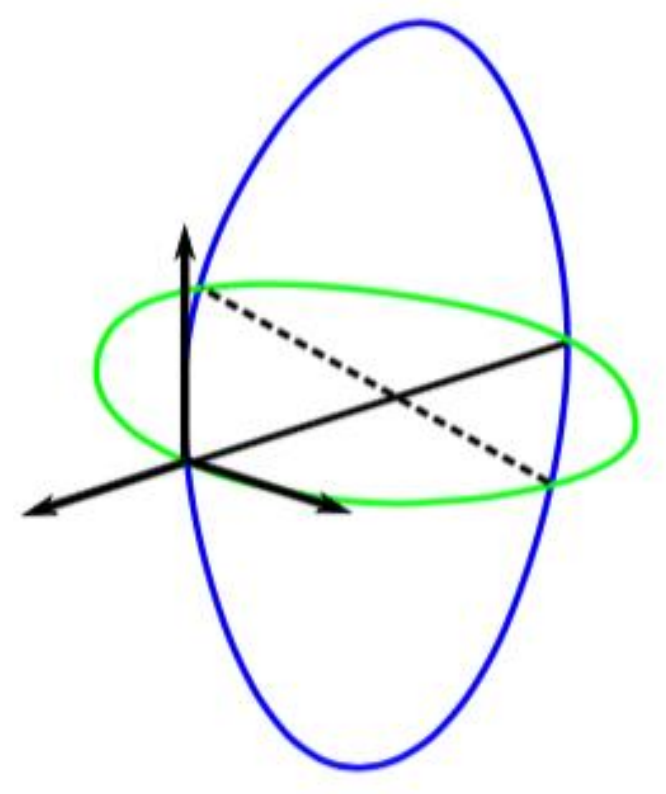




\section{Single-View Sketch-Based Modeling [Andre and Saito 2011]}

- Two perpendicular cross sections form the projection of a cubic corner (which is welldefined)
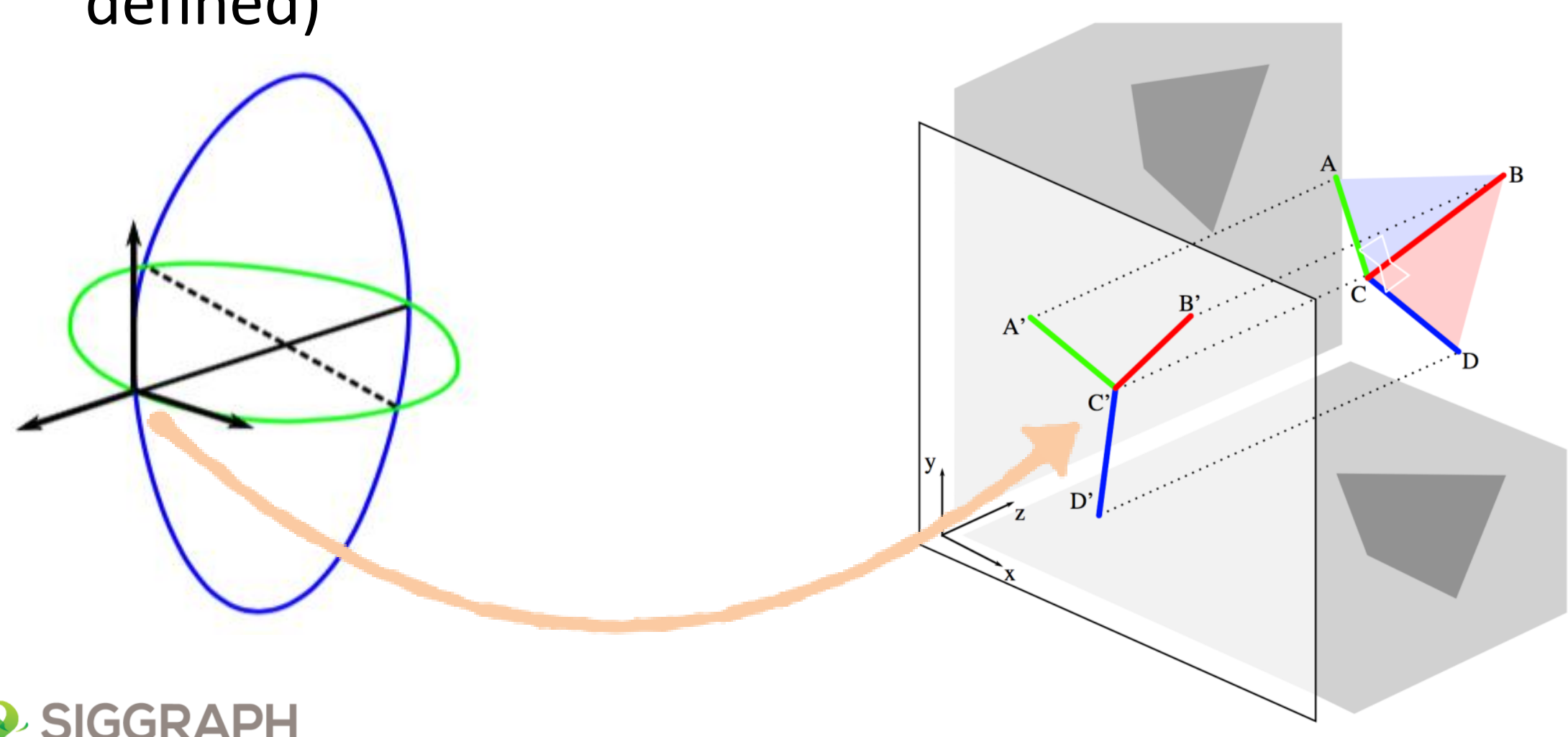


\section{Single-View Sketch-Based Modeling [Andre and Saito 2011]}

- That gives us 3D axes for the shape
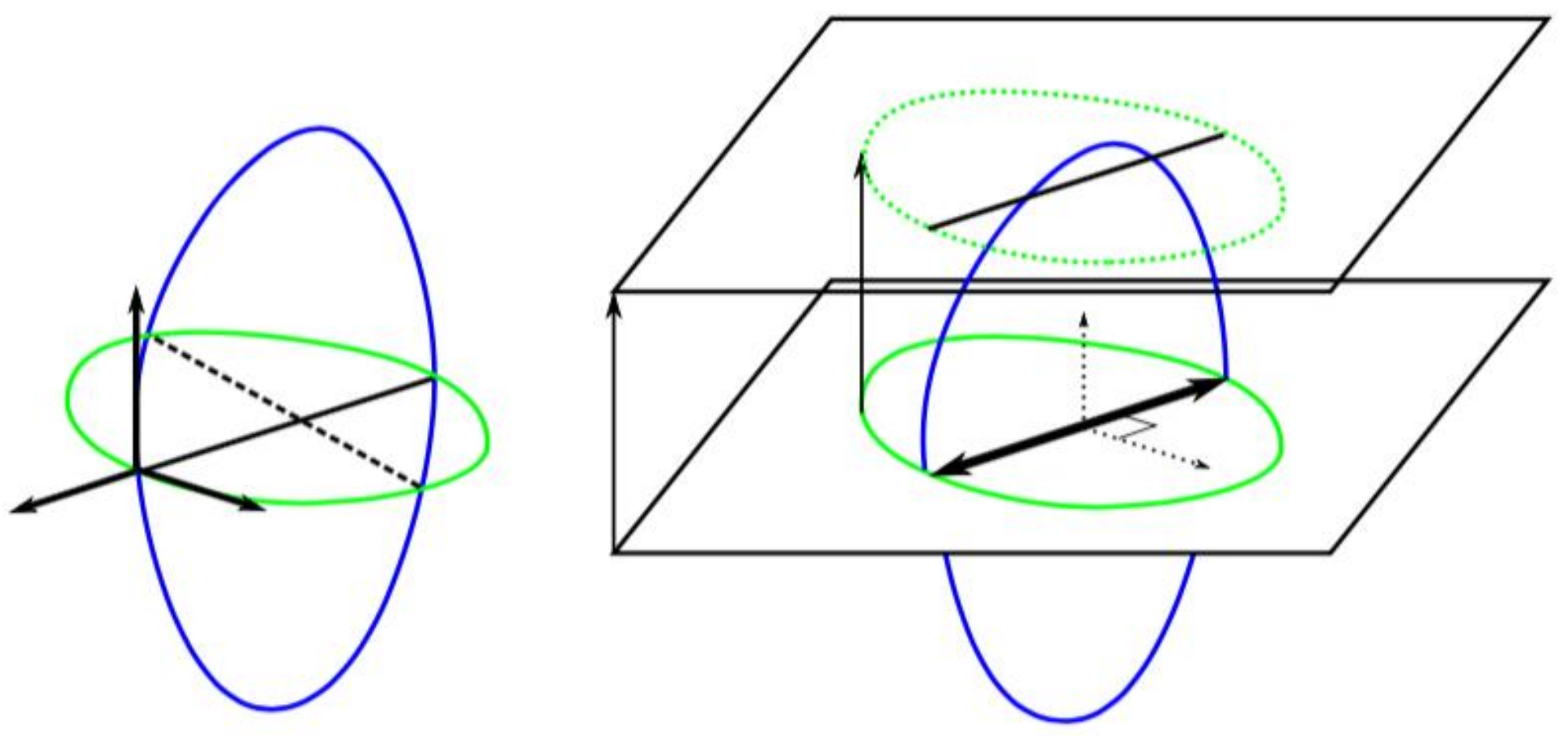

Q?. SIGGRAPH

- ASIA 2916 


\section{Single-View Sketch-Based Modeling [Andre and Saito 2011]}

- Which we can use to sweep out a surface
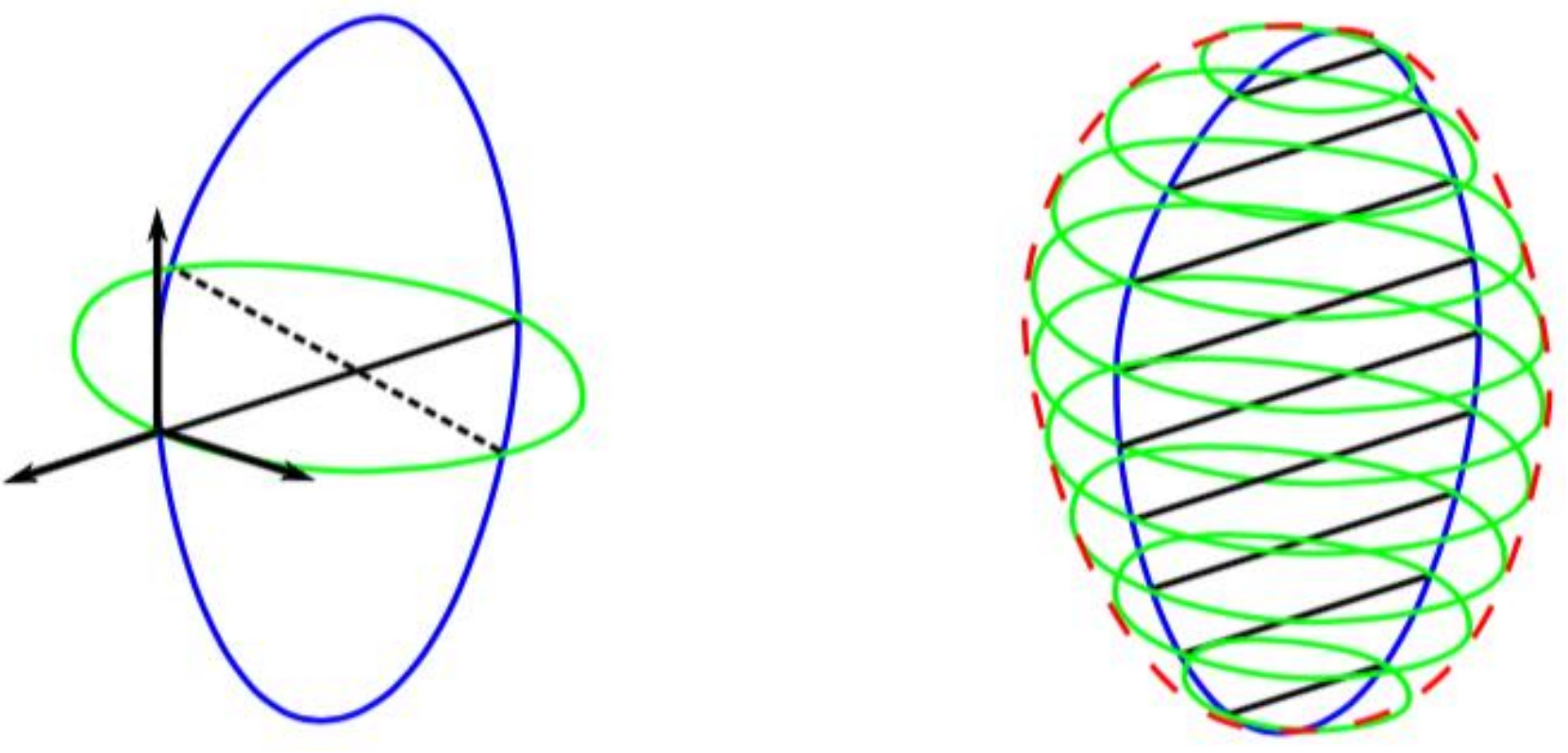


\section{Single-View Sketch-Based Modeling [Andre and Saito 2011]}
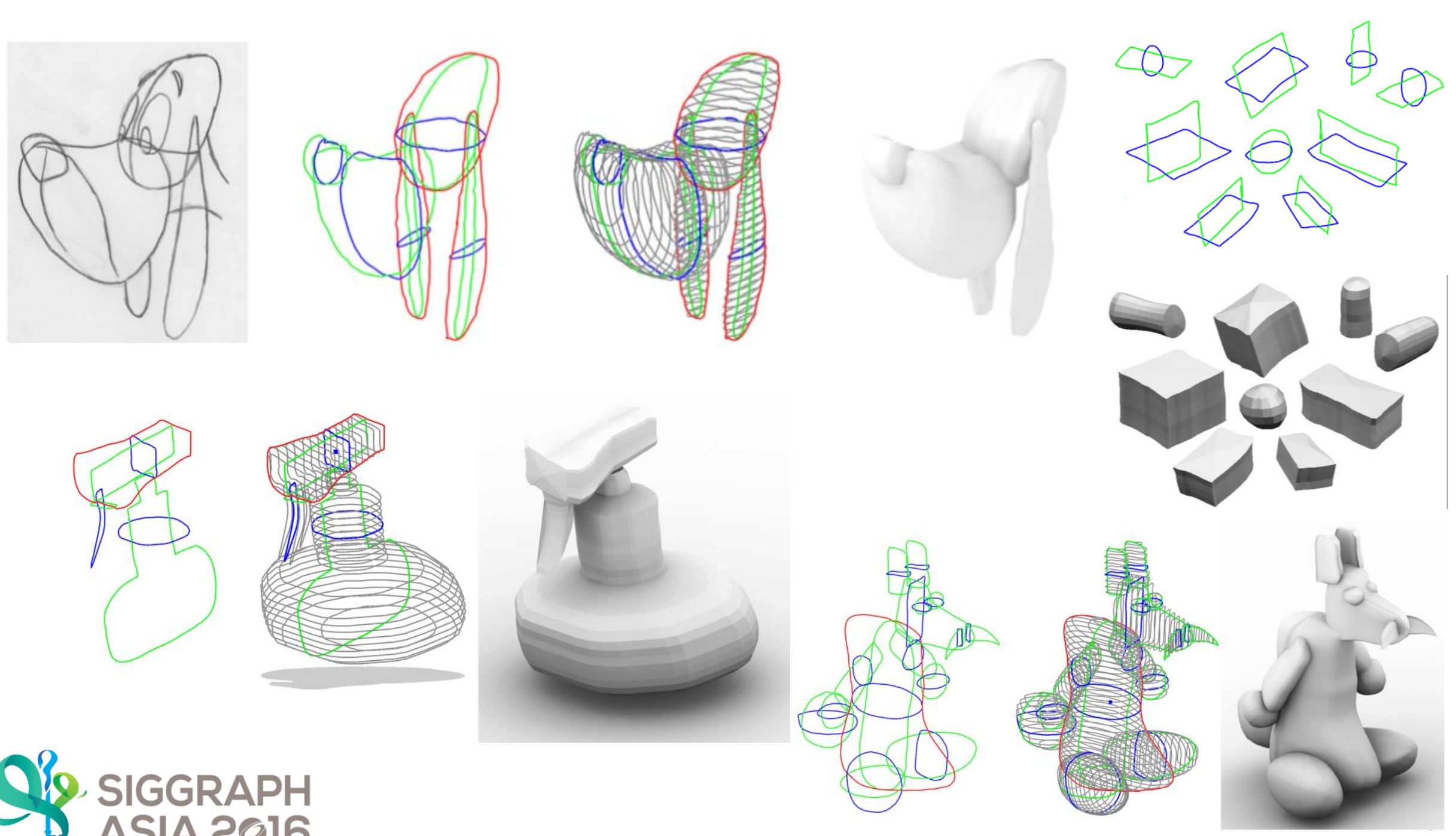

Q\%, SIGGRAPH

ASIA 2016
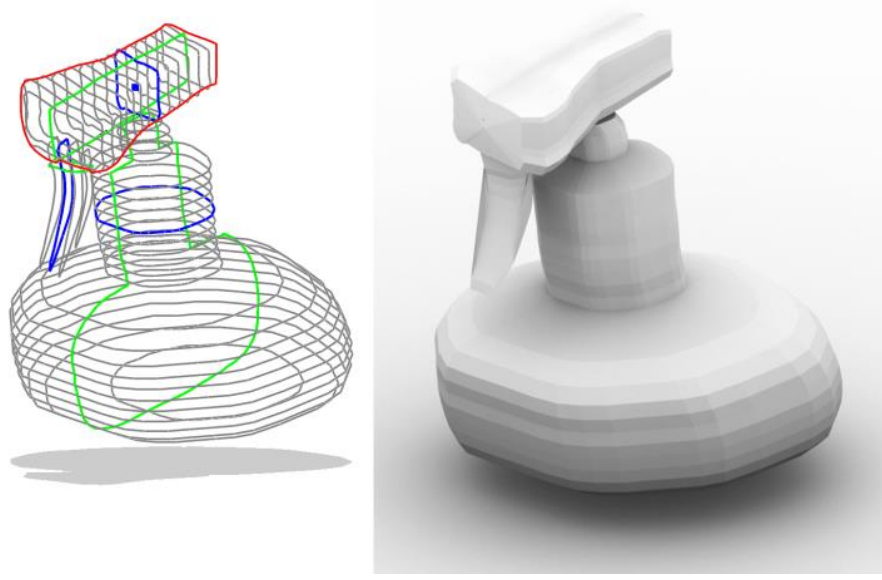


\section{A suggestive interface for image guided 3D sketching [Tsang et al. 2004]}

- Use the guide sketch to snap strokes.

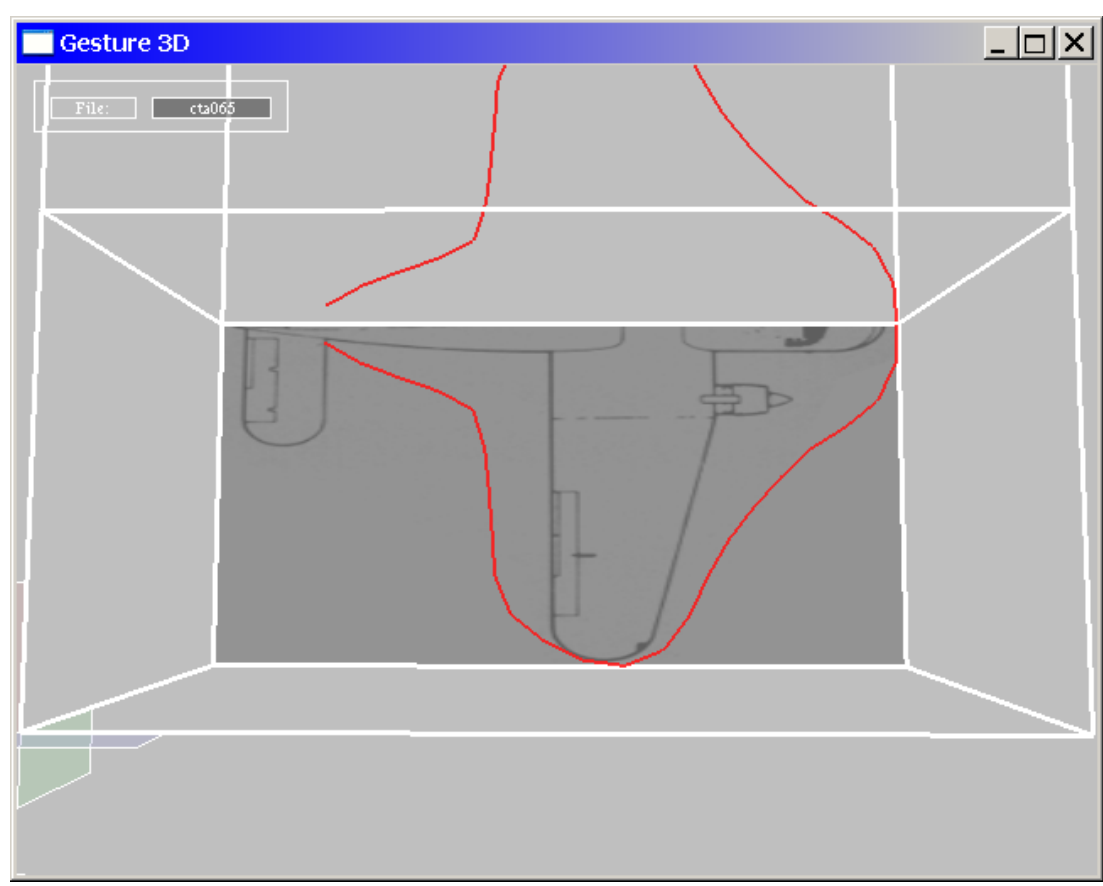

User sketch

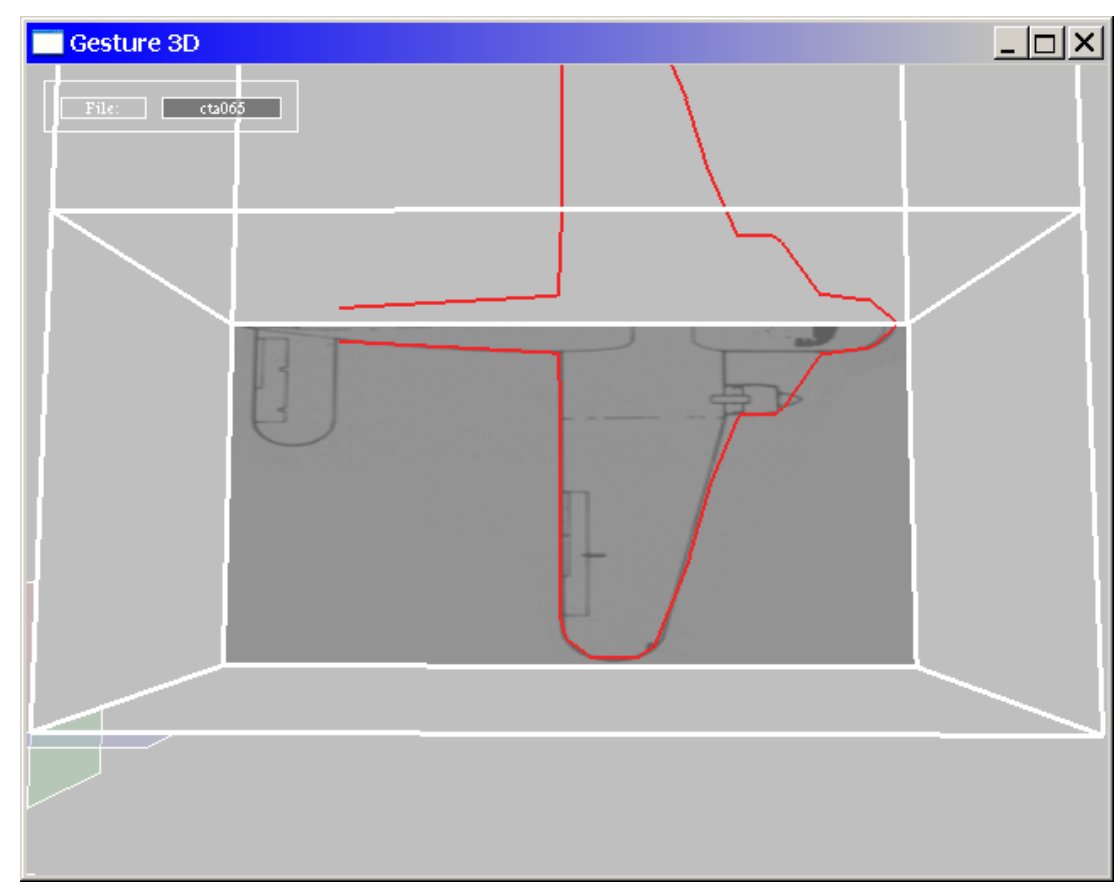

Automatically snapped to the guide image 


\section{Challenges}

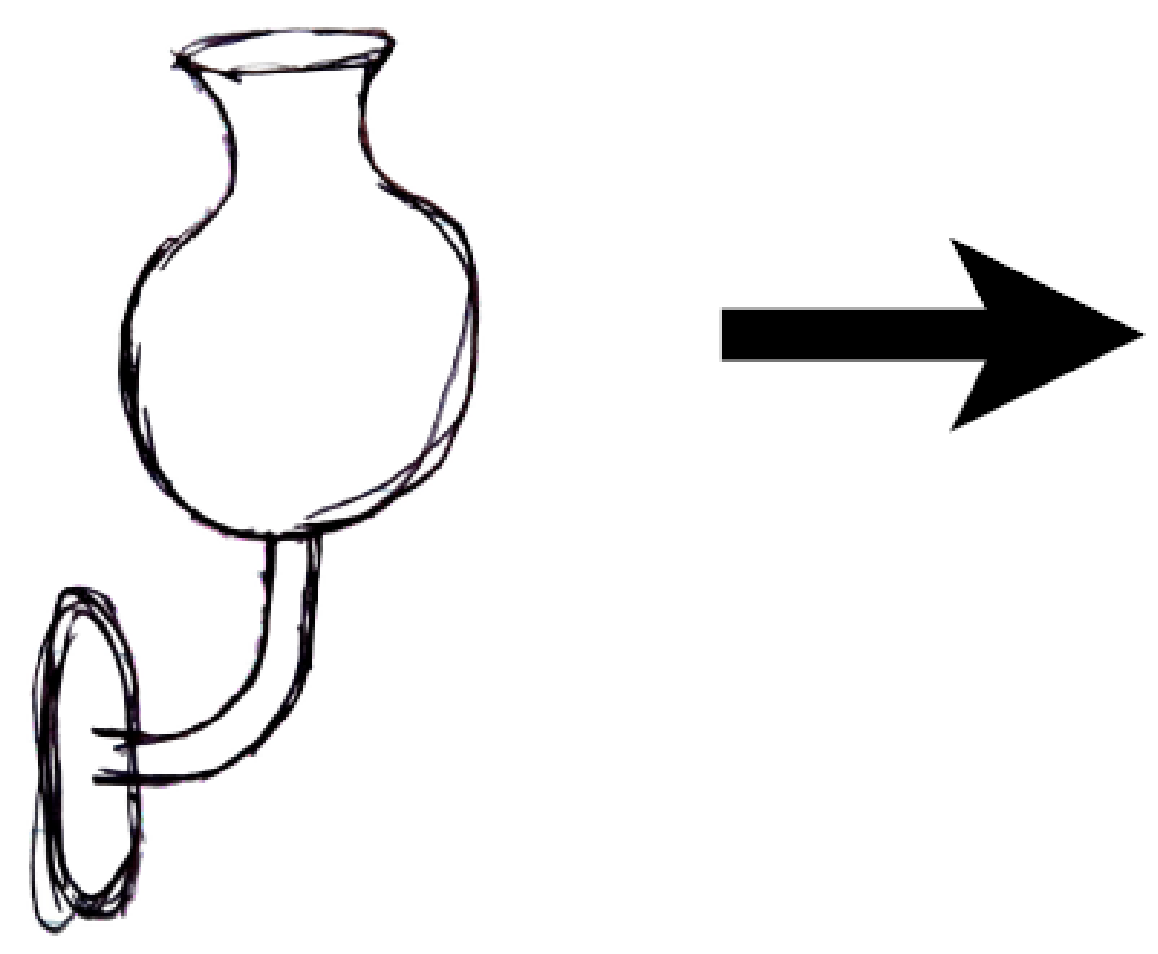

QQ. SIGGRAPH

ASIA 2016 


\section{Challenges}
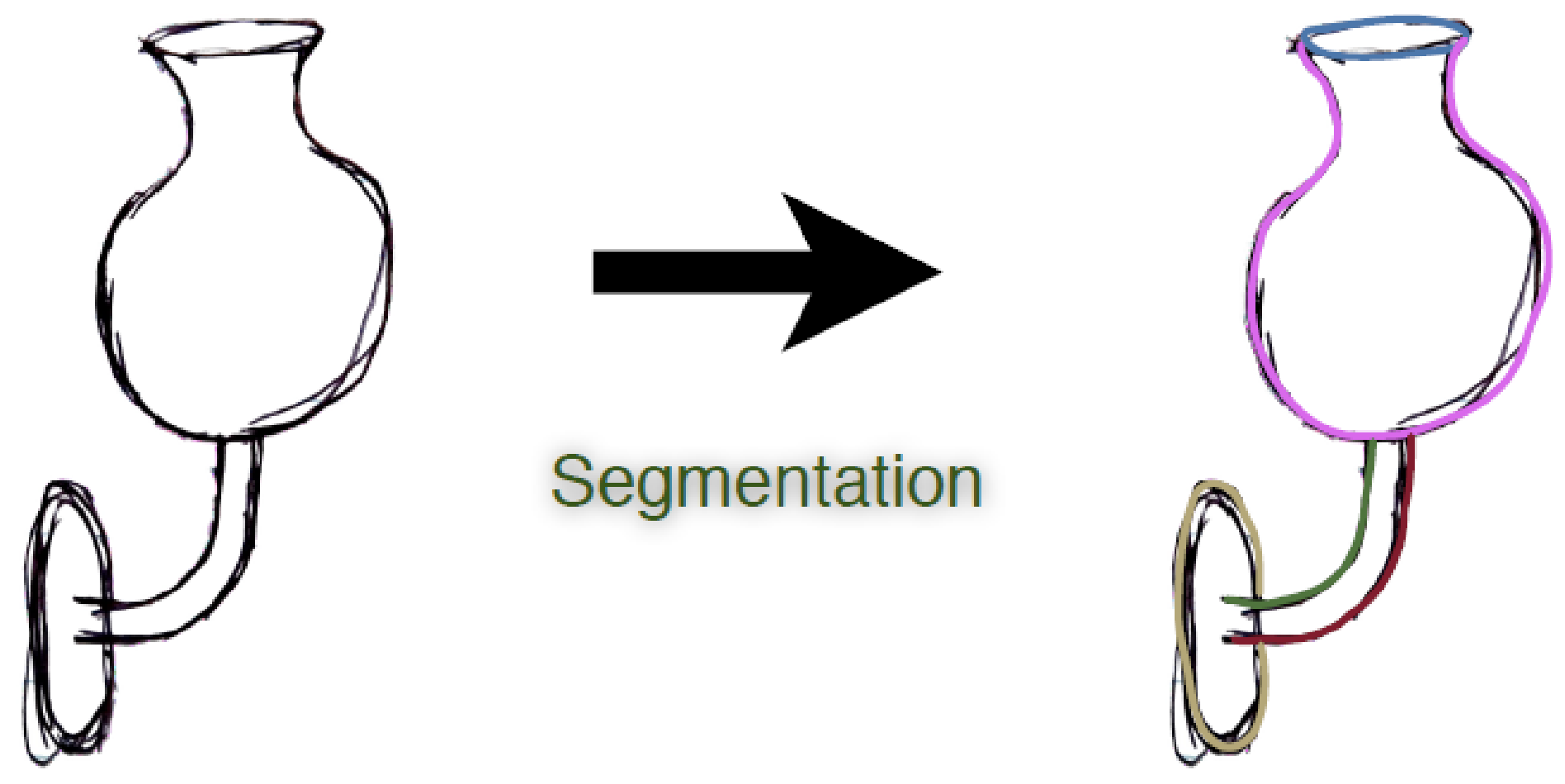

QQ, siggraPh

- ASIA 2916 


\section{Challenges}
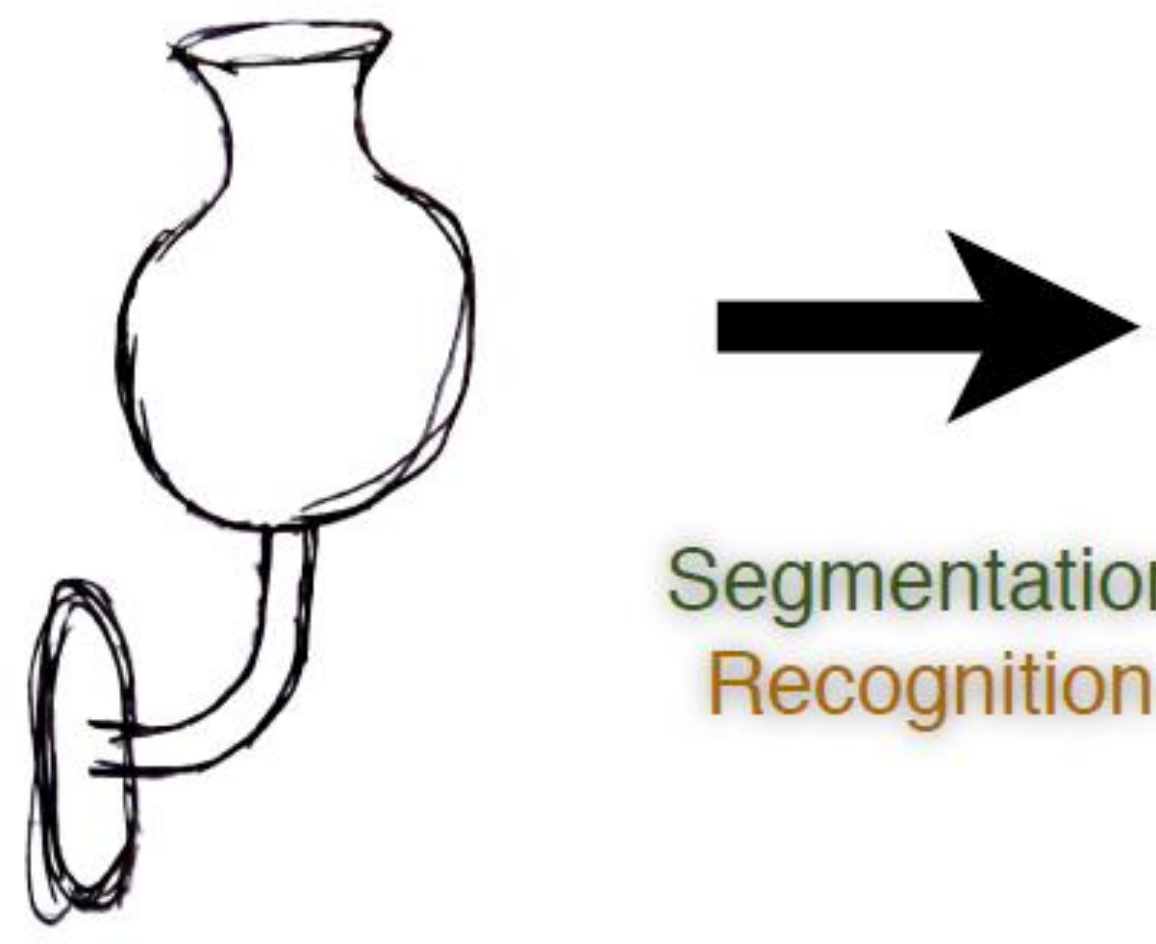

Segmentation Recognition

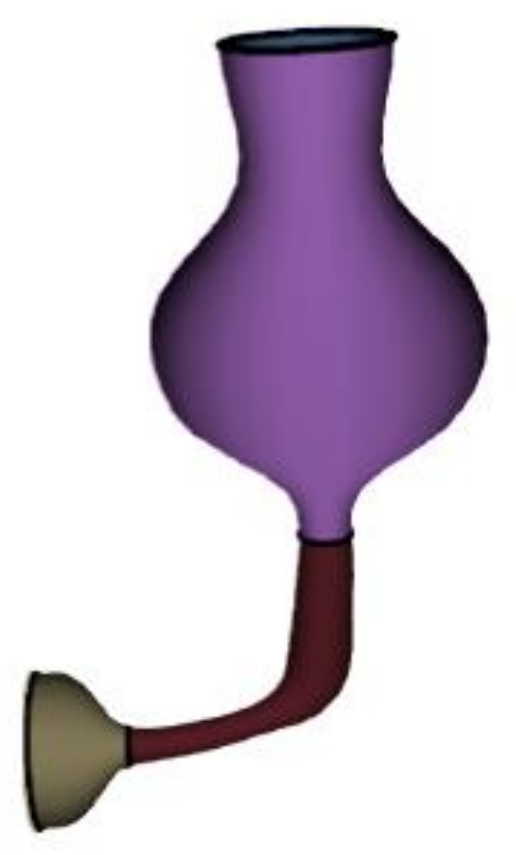

QQ, siggraPh 


\section{Challenges}
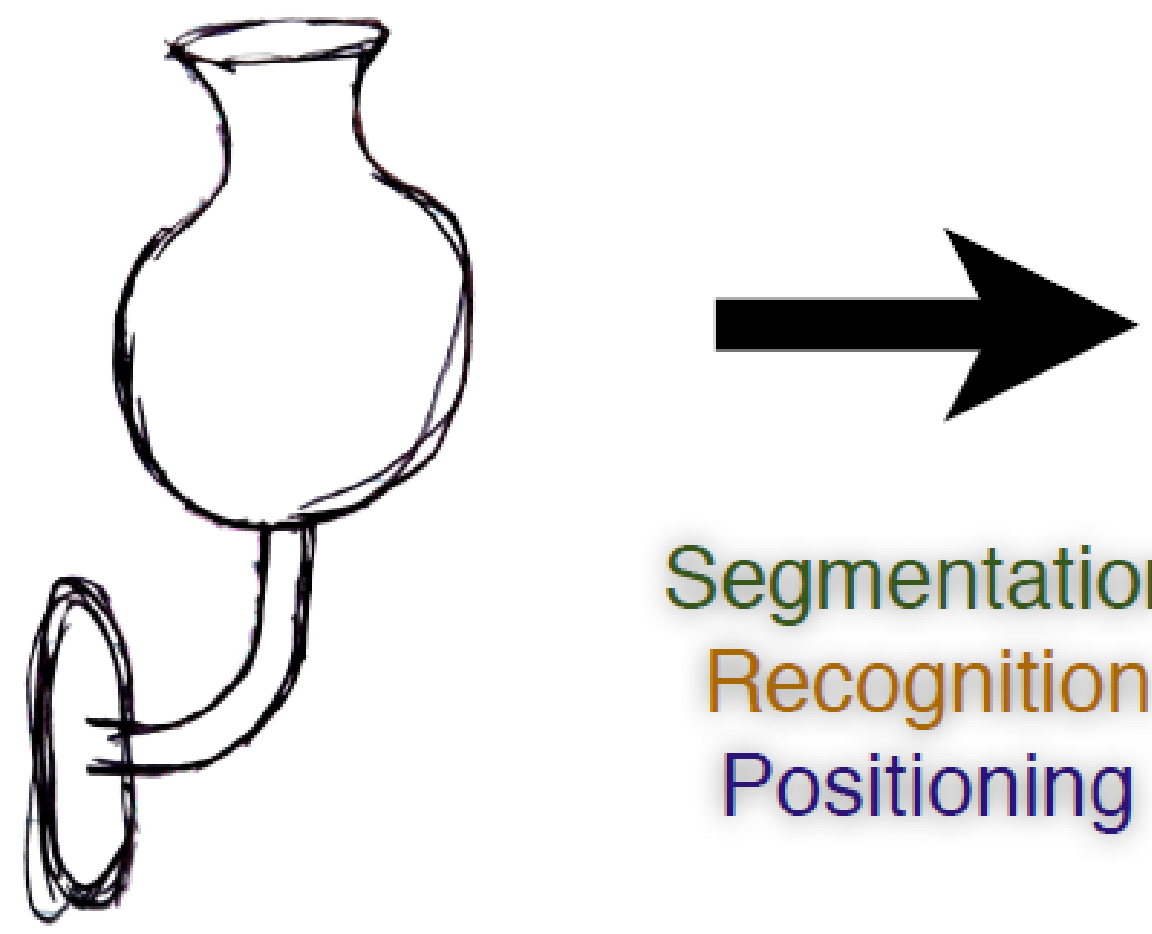

Segmentation Recognition Positioning

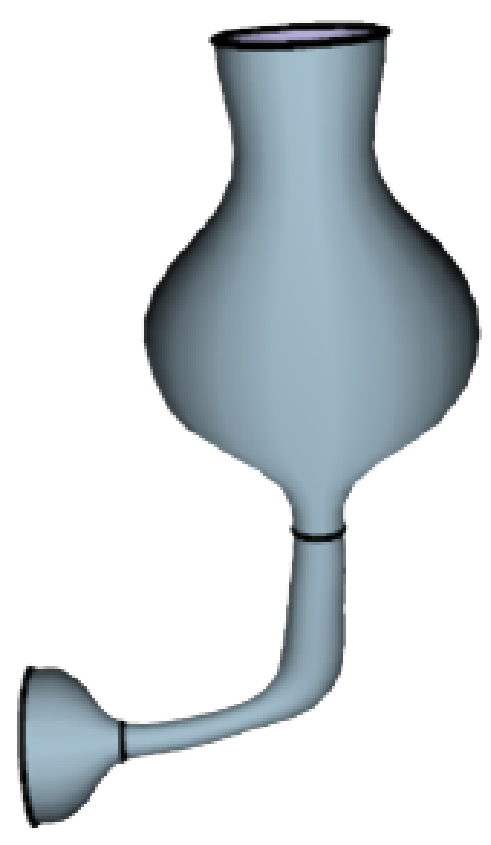

An automatic solution entails solving a complex, nonconvex optimization problem with many local minima. 


\section{Interactive Approach}

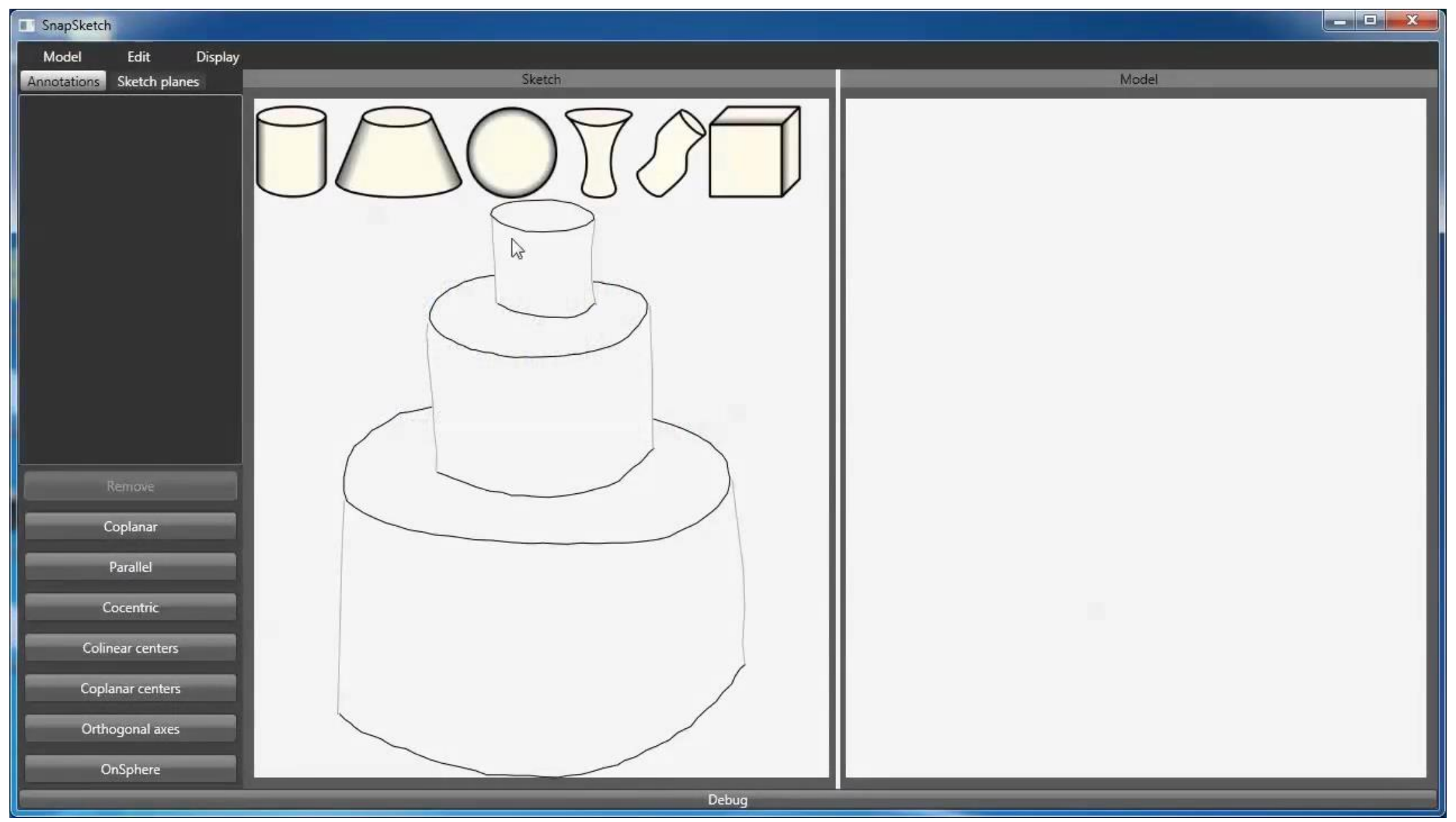

Q 2 , SIGGRAPH

AS ASIA 2016 
Overview

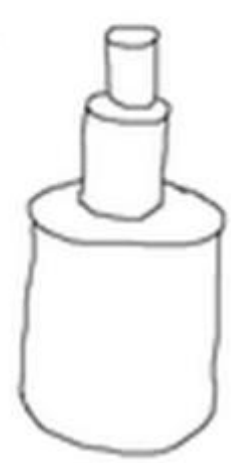

input

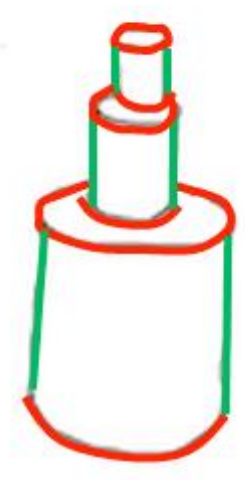

semantic
classification

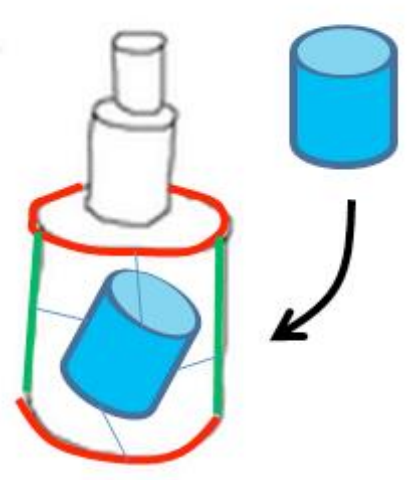

interactive
matching

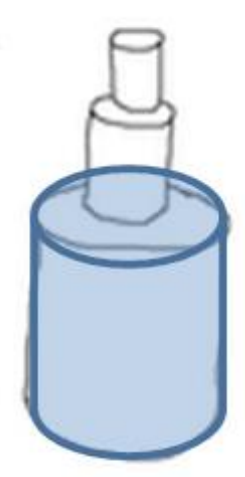

real-time
snapping

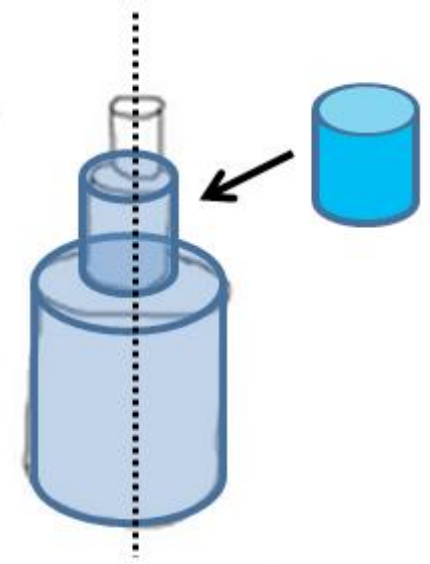

geosemantic

snapping

26 


\section{Primitives:}

\section{Feature Curves}

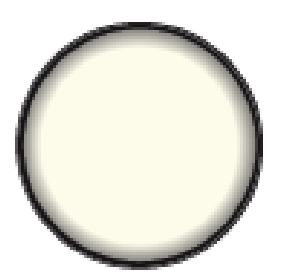

sphere

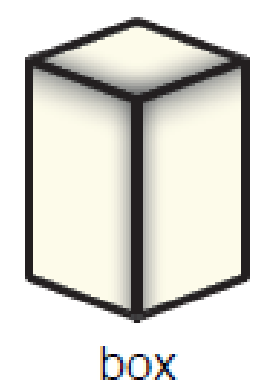

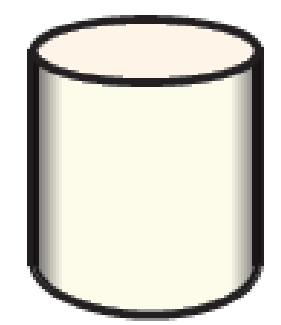

straight cylinder

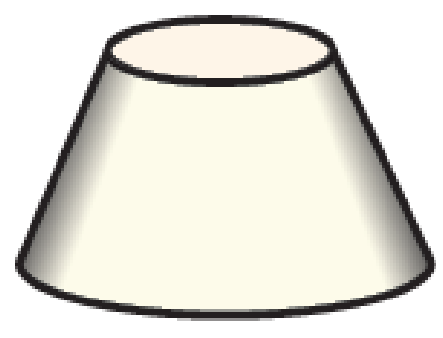

truncated cone

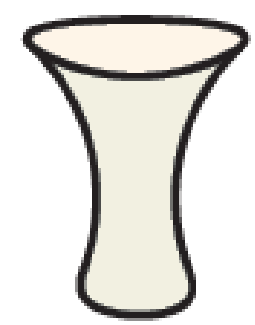

straight generalized

cylinder

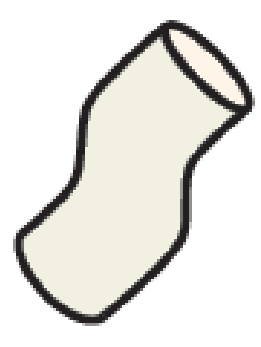

bent

generalized

cylinder 


\section{Primitives:}

\section{Feature Curves}

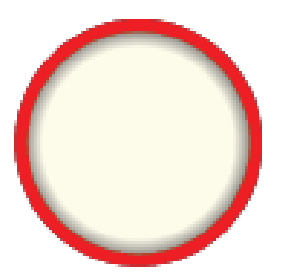

sphere

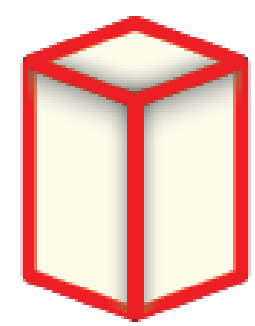

box

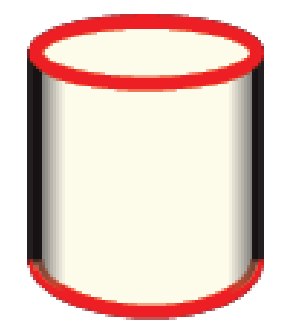

straight cylinder

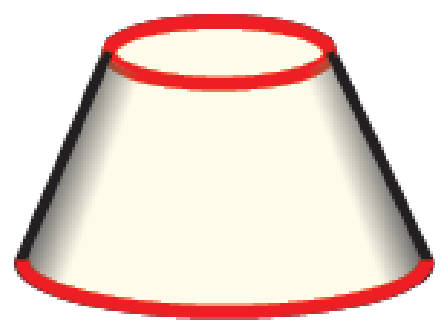

truncated cone

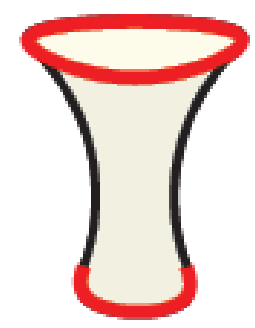

straight generalized

cylinder

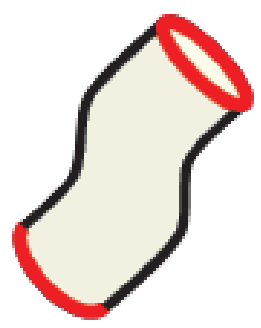

bent

generalized

cylinder 


\section{Primitives:}

\section{Silhouette Curves}

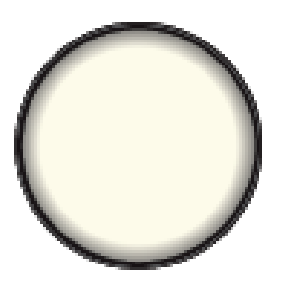

sphere

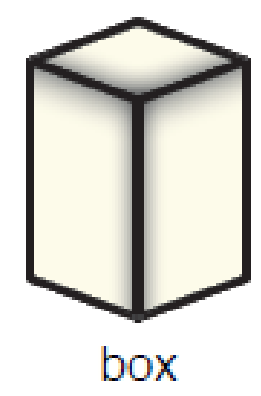

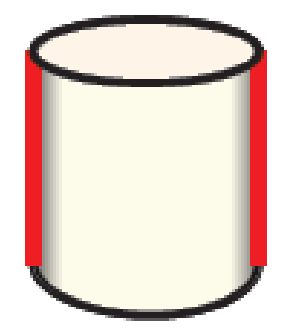

straight cylinder

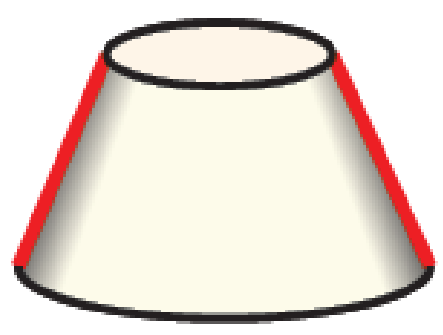

truncated cone

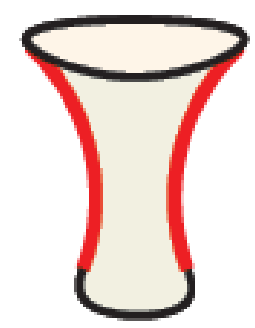

straight generalized

cylinder

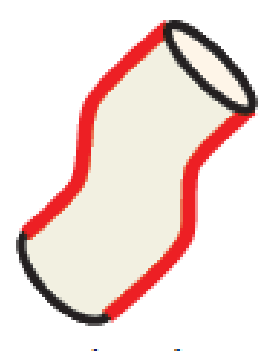

bent

generalized

cylinder 


\section{Tagging}

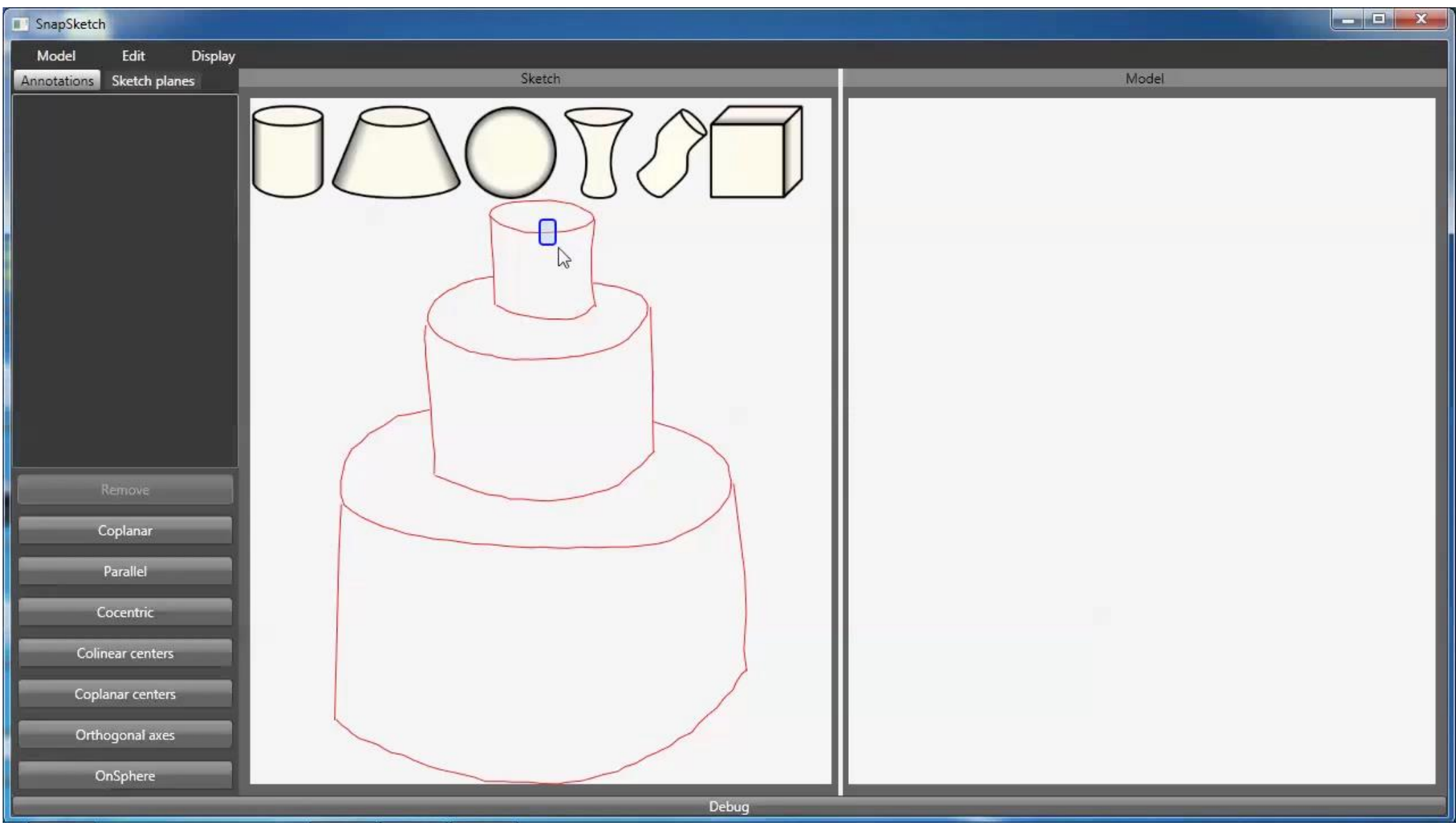




\section{Tagging}

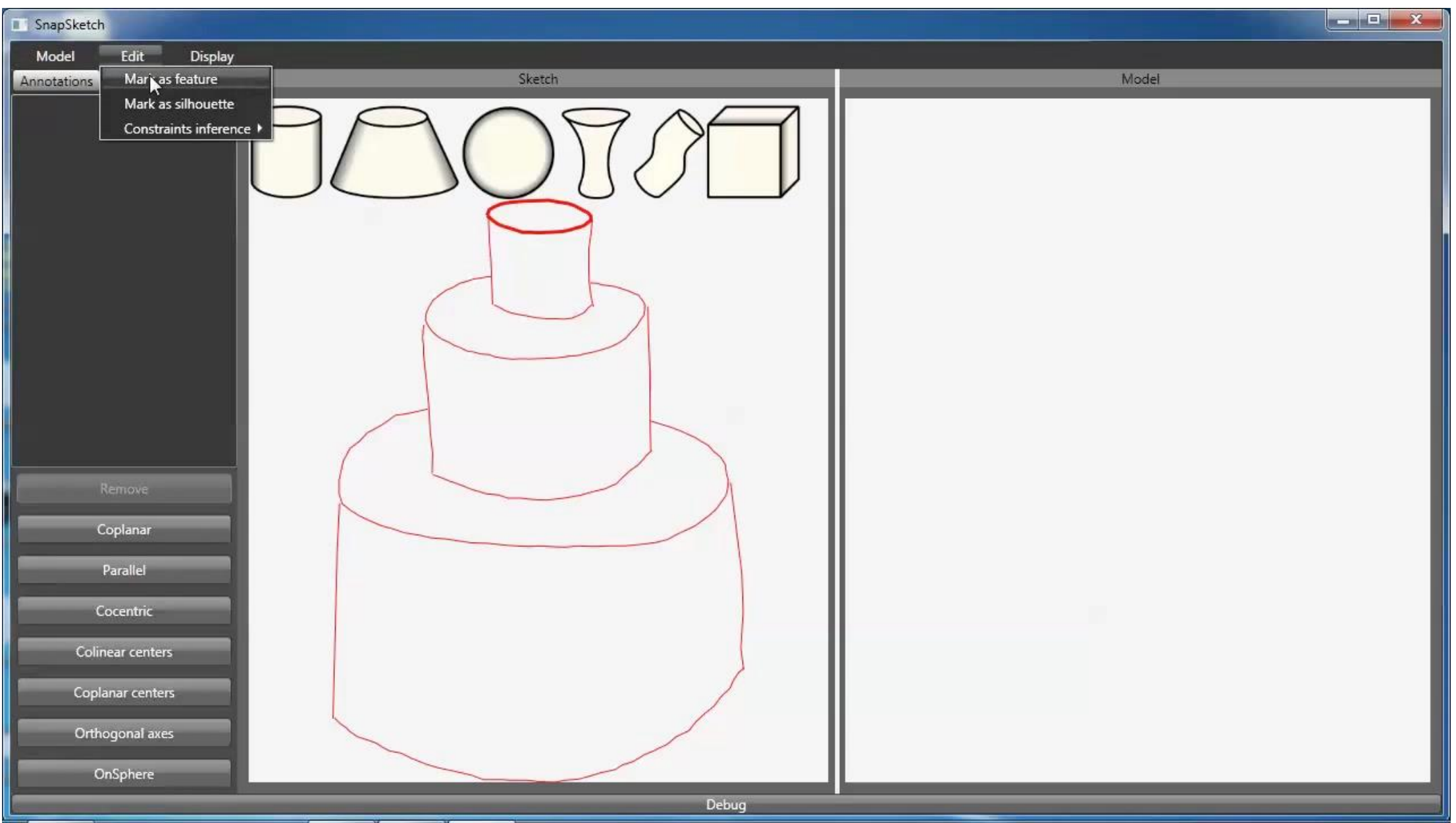




\section{Tagging}

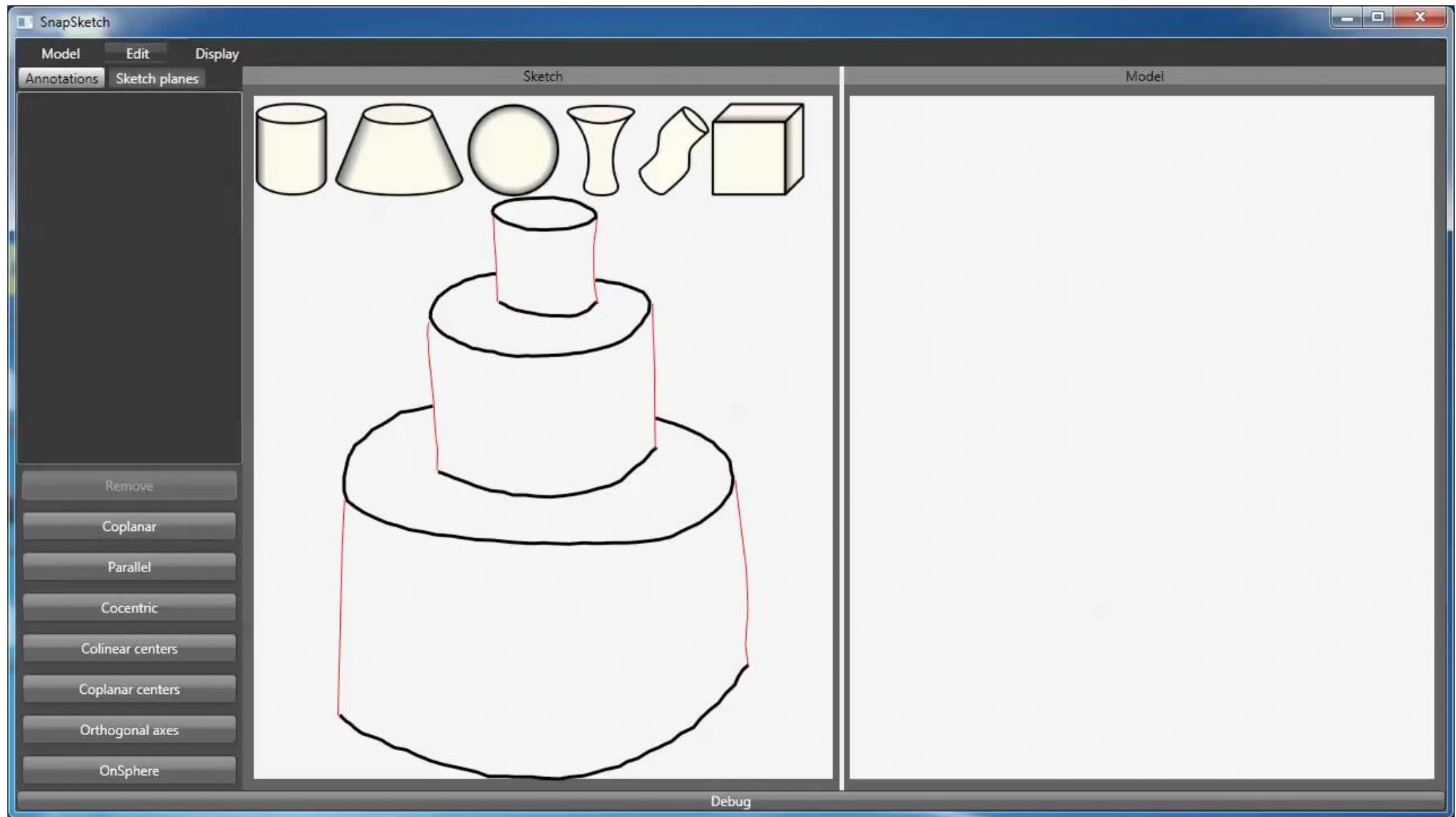

Q 2 , SIGGRAPH

- ASIA 2916 


\section{Drag-and-Drop}

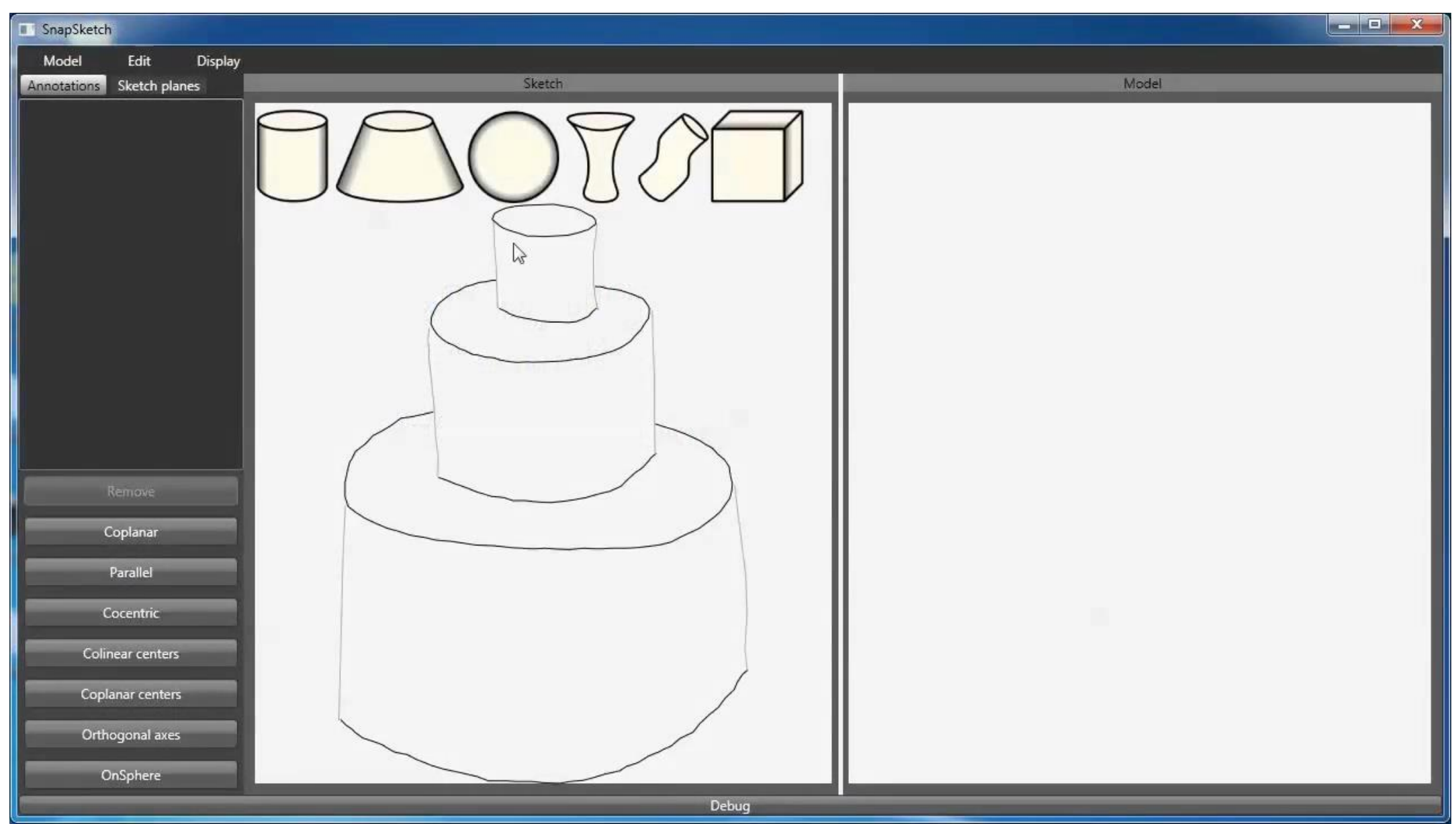

Q 2 , SIGGRAPH

ASIA 2016 


\section{Anatomy of a Drag: Curve Matching}

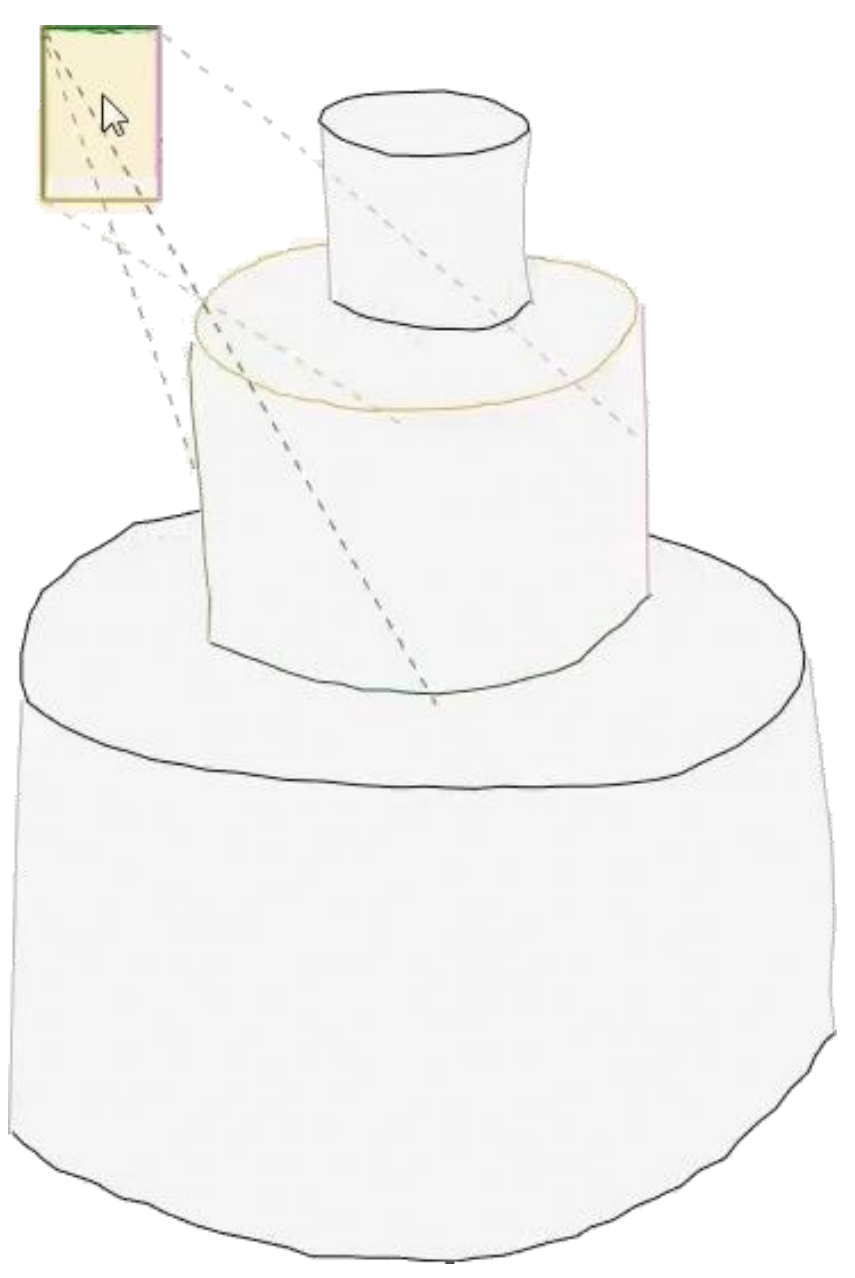




\section{Anatomy of a Drag: Curve Matching}

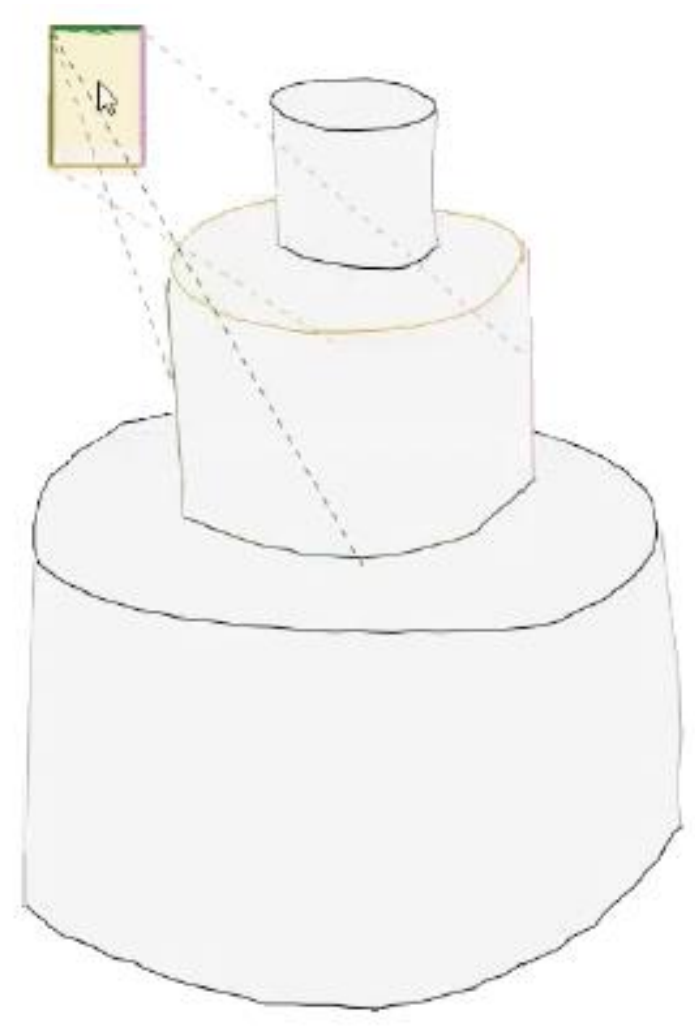




\section{Anatomy of a Drag: Curve Matching}

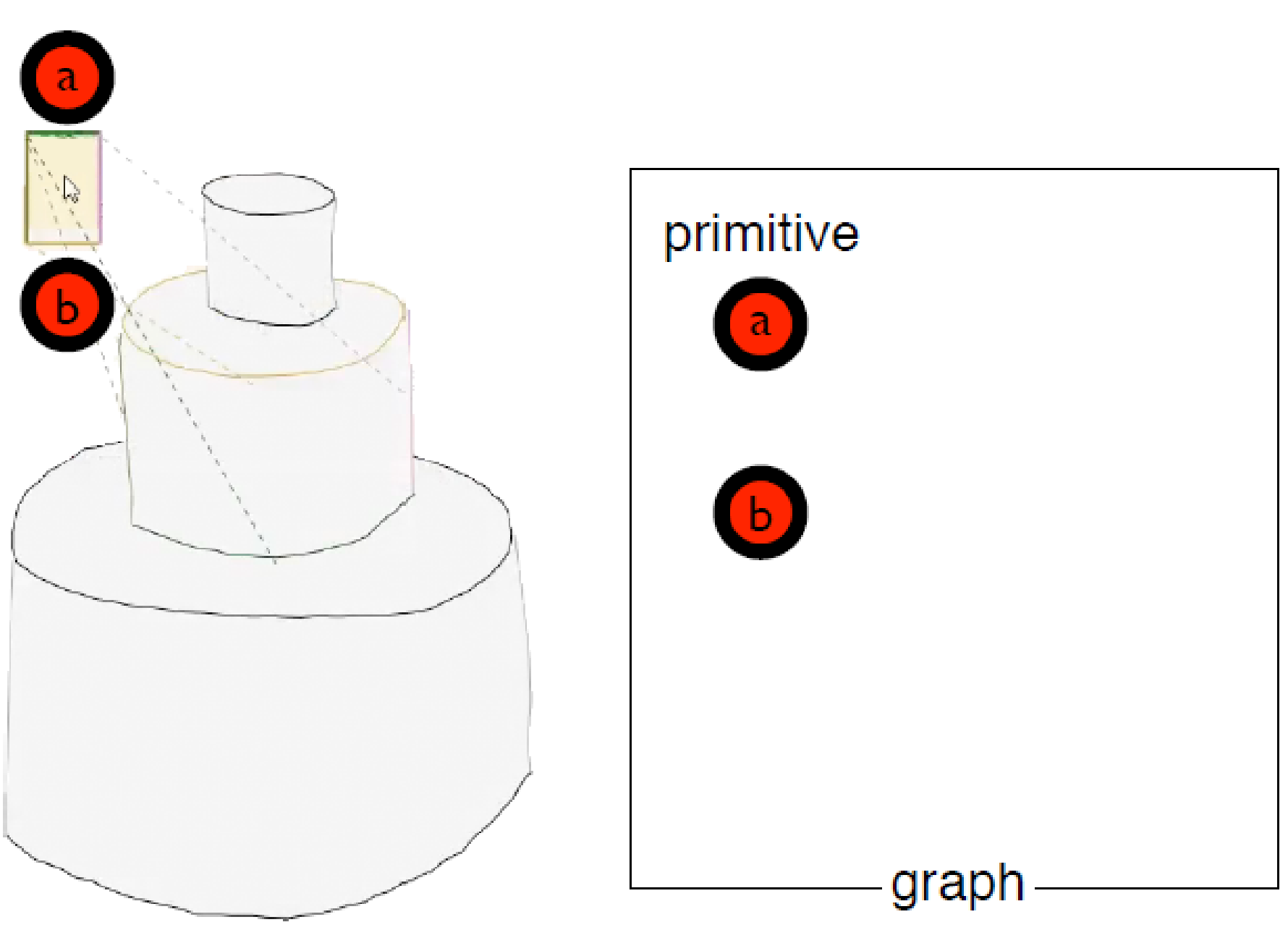




\section{Anatomy of a Drag: Curve Matching}

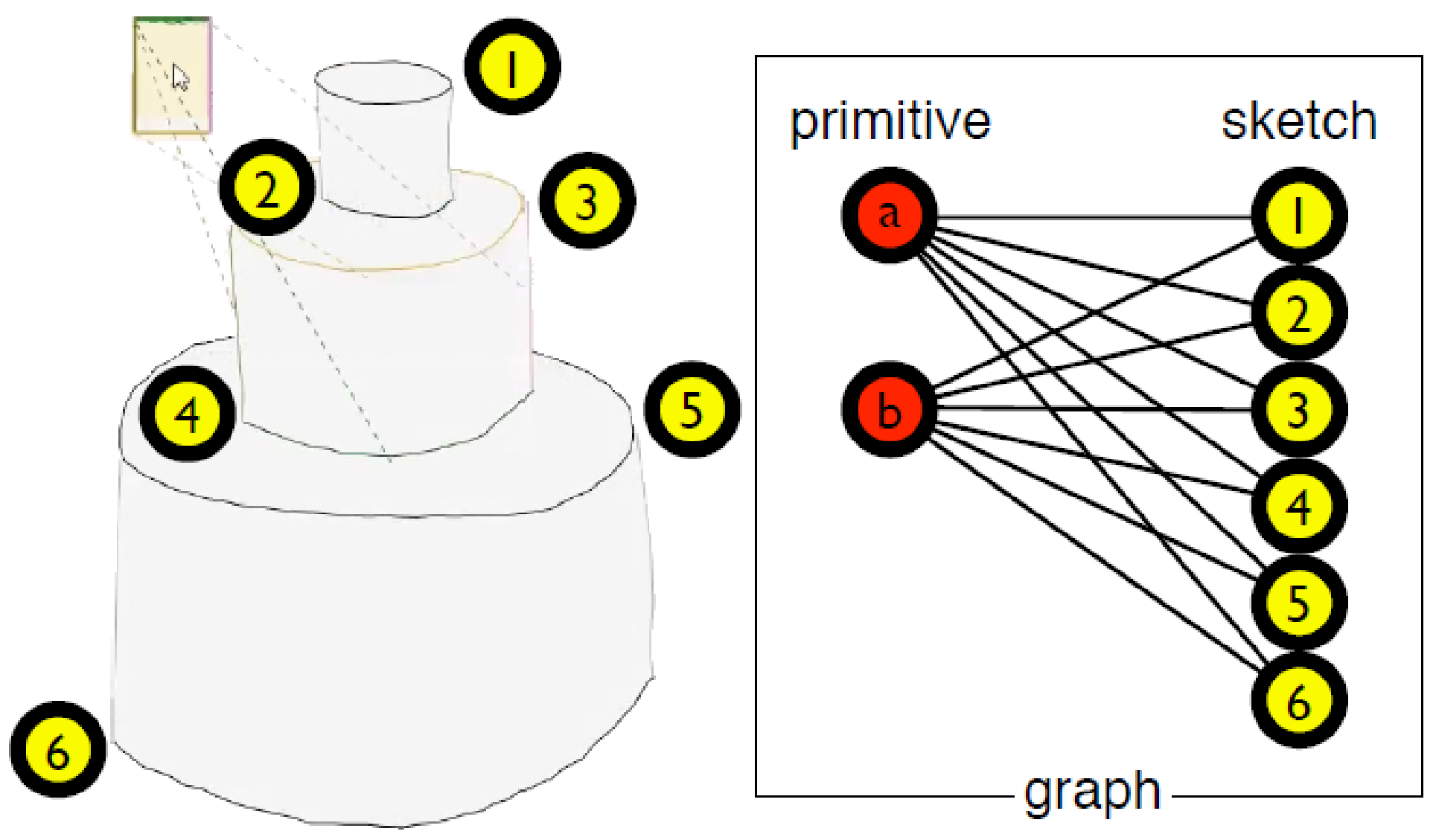

Q9. SIGGRAPH 


\section{Anatomy of a Drag: Curve Matching}
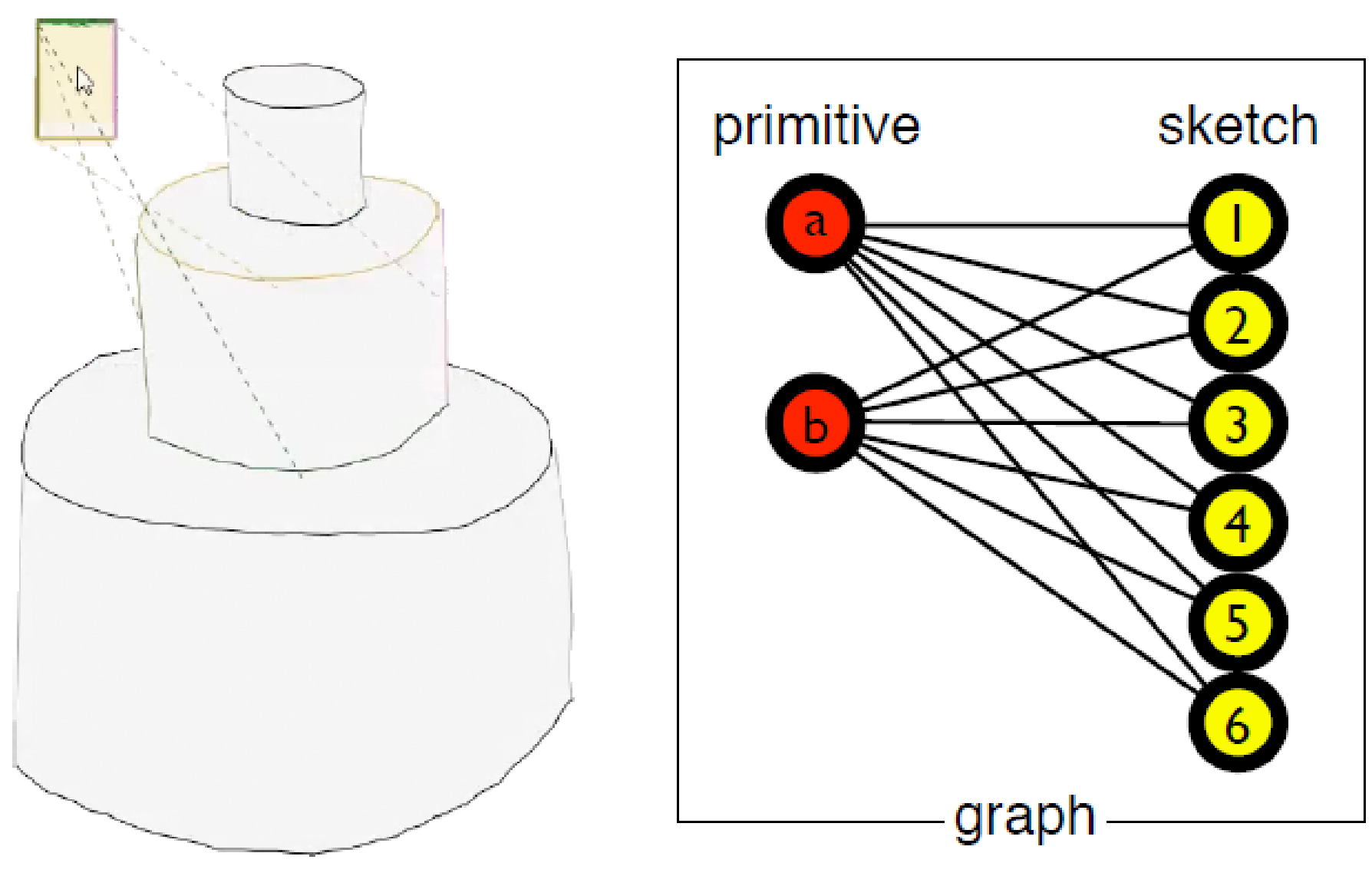

Q? SIGGRAPH 


\section{Anatomy of a Drag: Curve Matching}

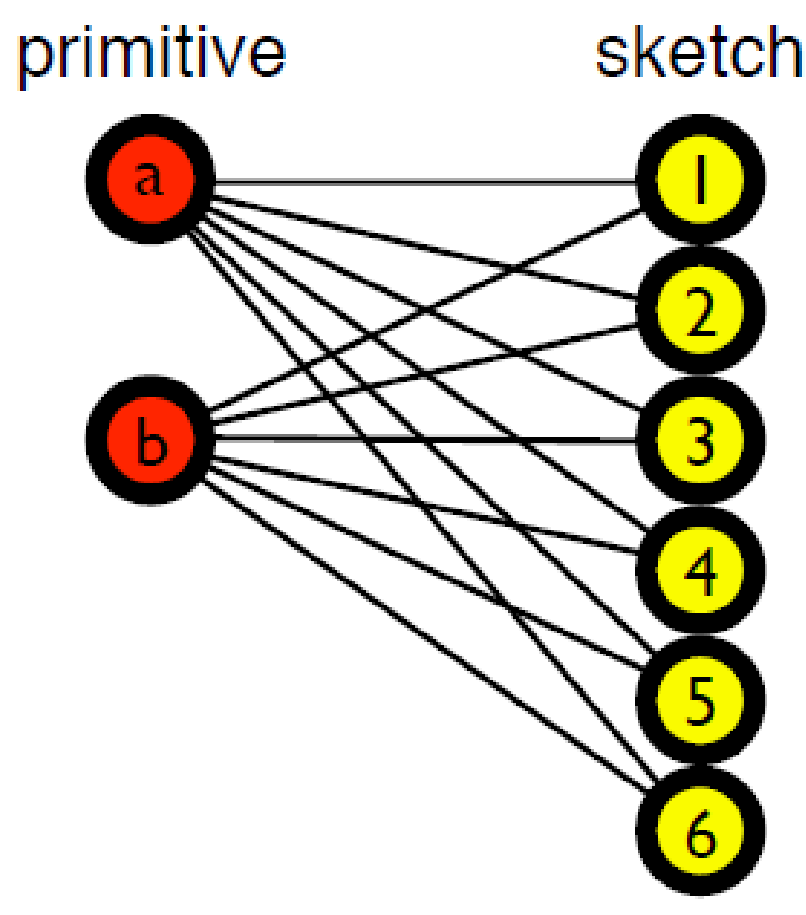




\section{Anatomy of a Drag: Curve Matching}
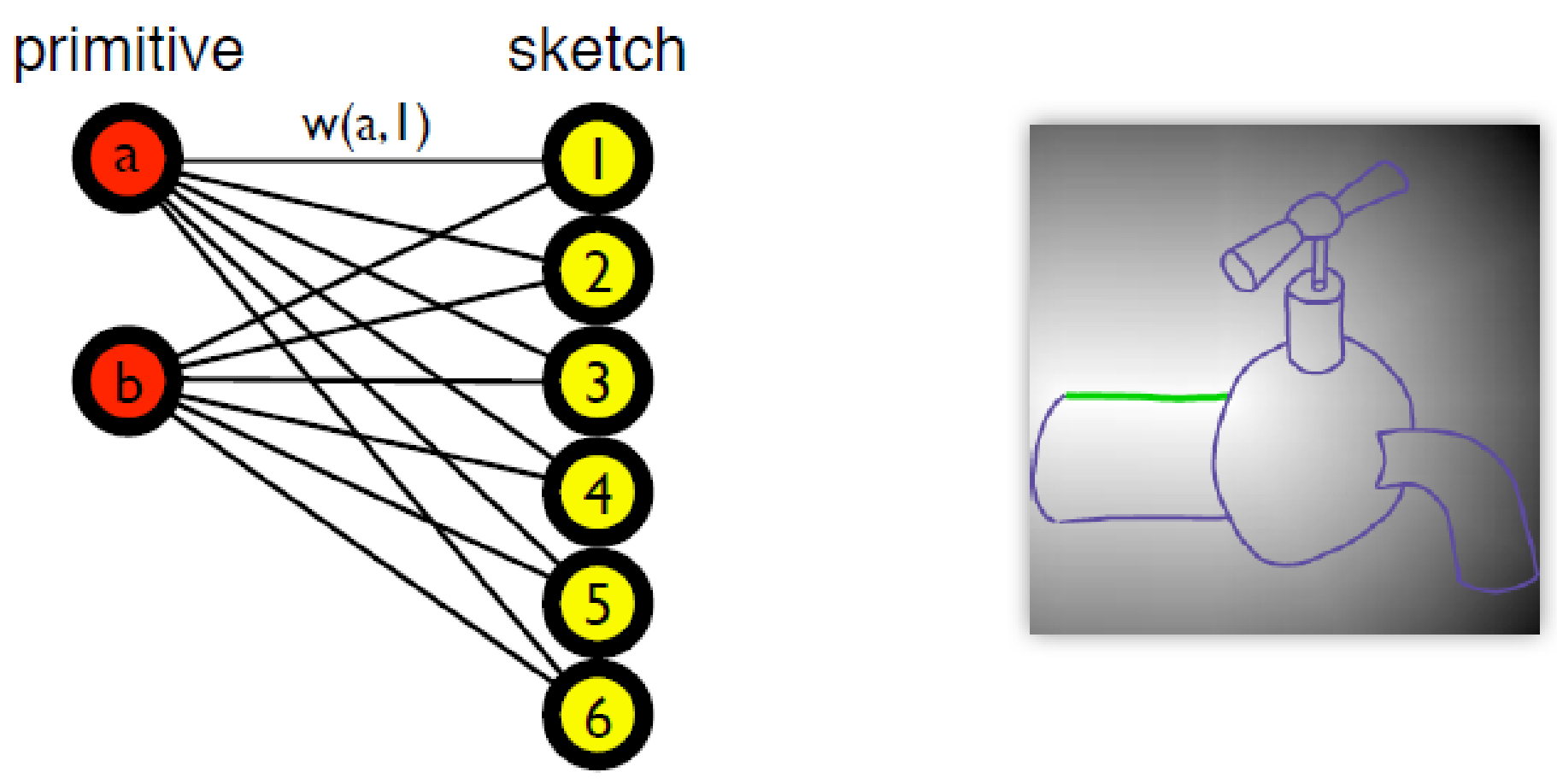

QQ, SIGGRAPH 


\section{Anatomy of a Drag: Curve Matching}

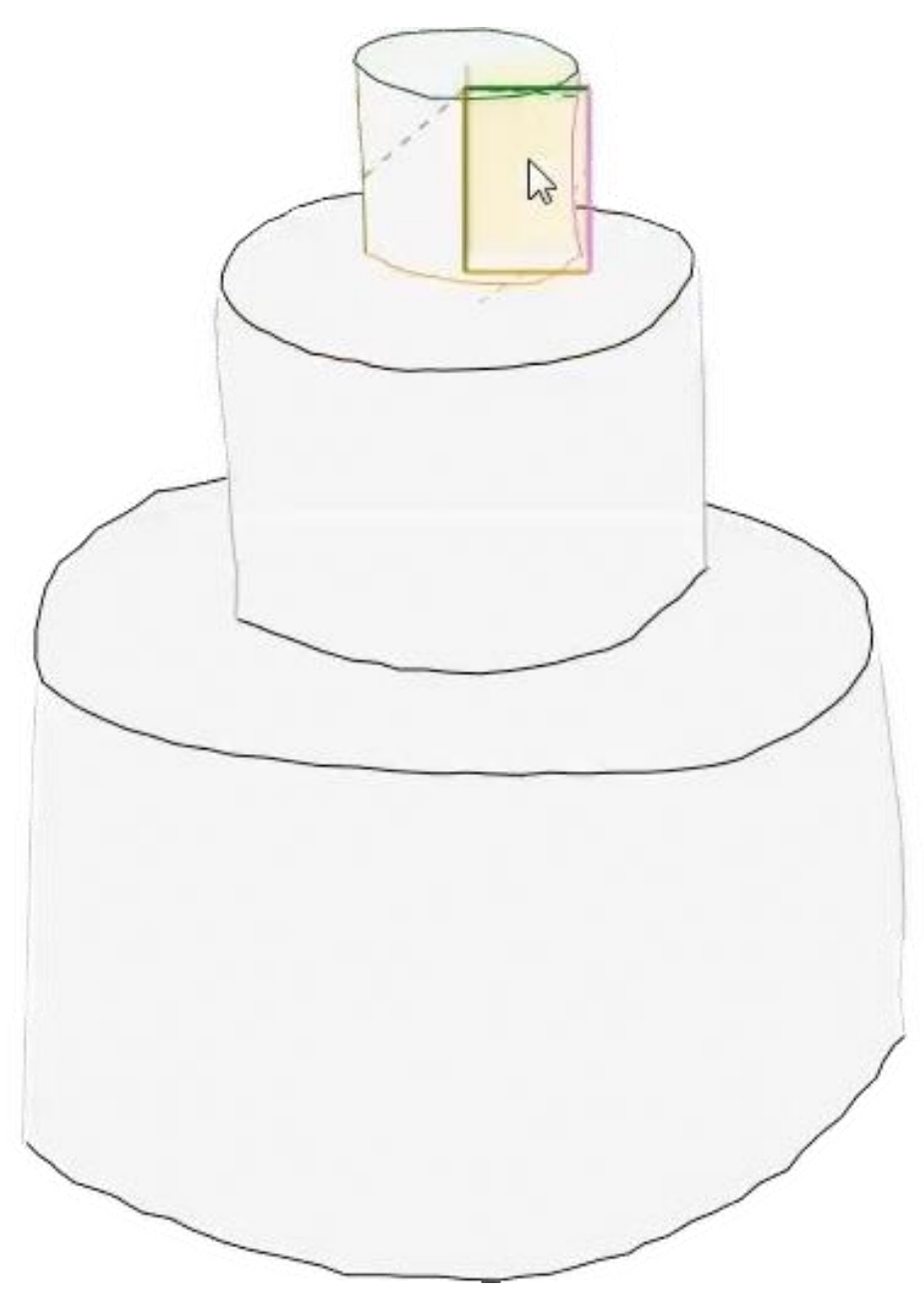




\section{Anatomy of a Drag: Primitive Fitting}

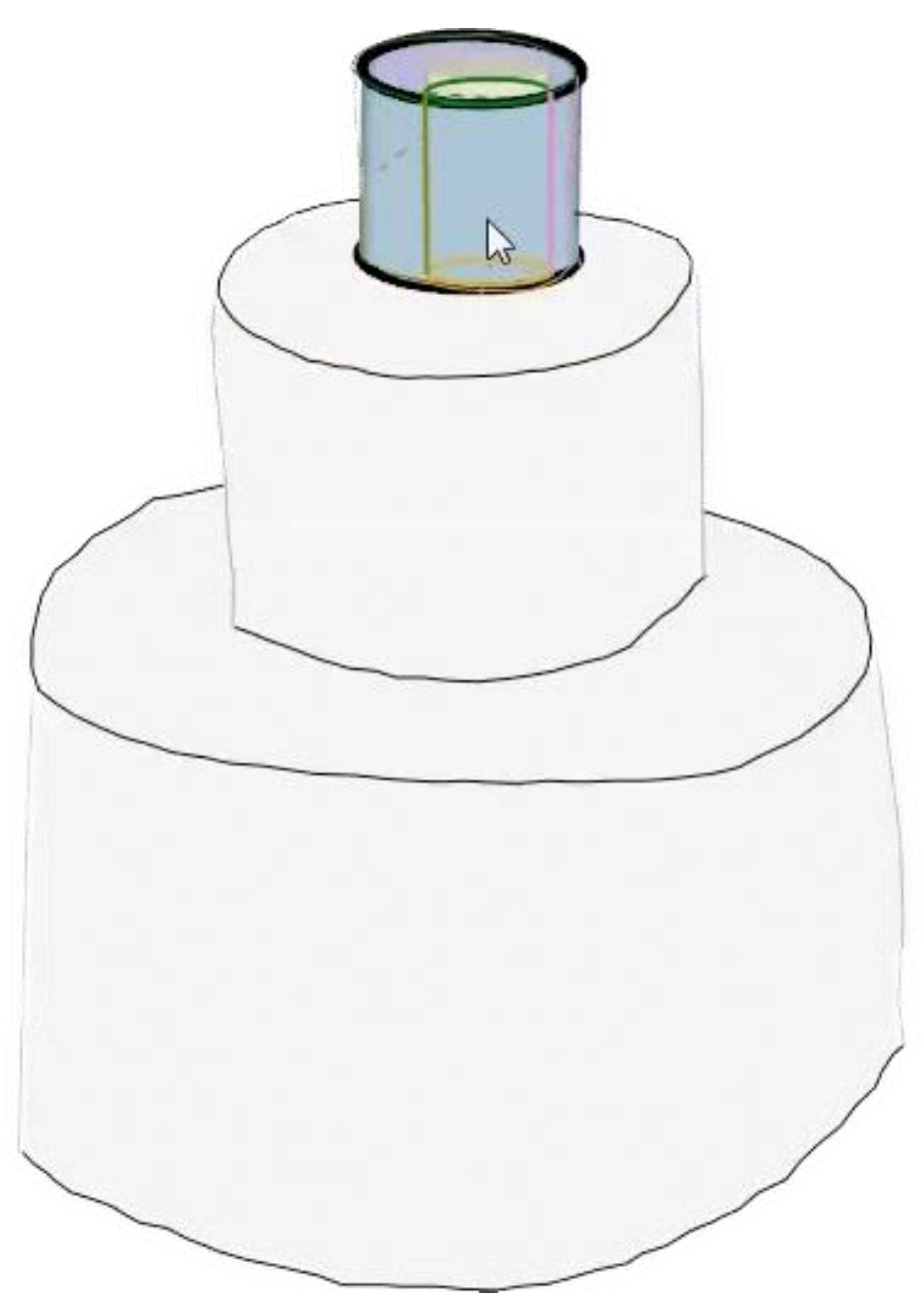




\section{Anatomy of a Drag: Primitive Fitting}

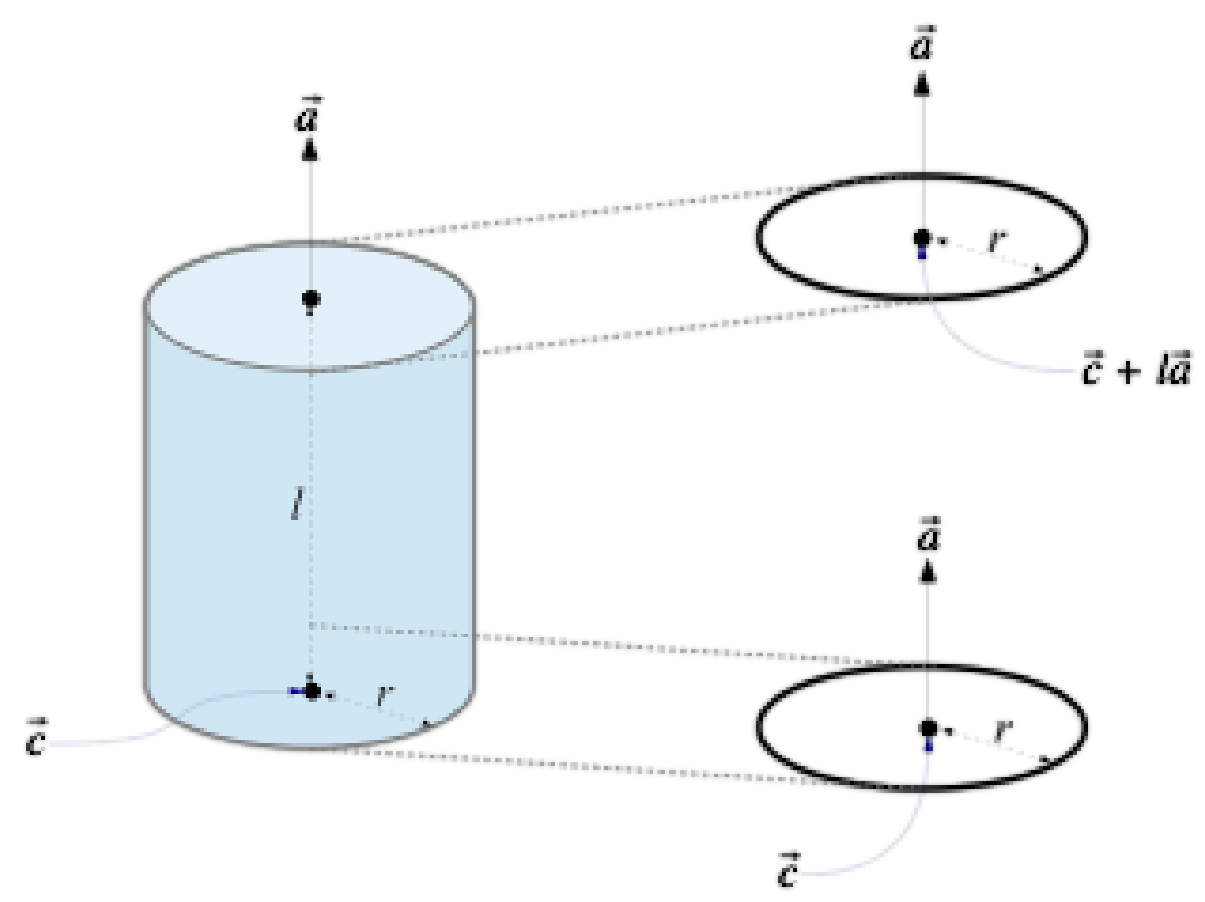

$$
\begin{aligned}
\min : & \phi_{p}\left(x_{p}\right) \\
\text { s.t. }: & C_{p}\left(x_{p}\right)=0
\end{aligned}
$$




\section{Anatomy of a Drag: Primitive Fitting}

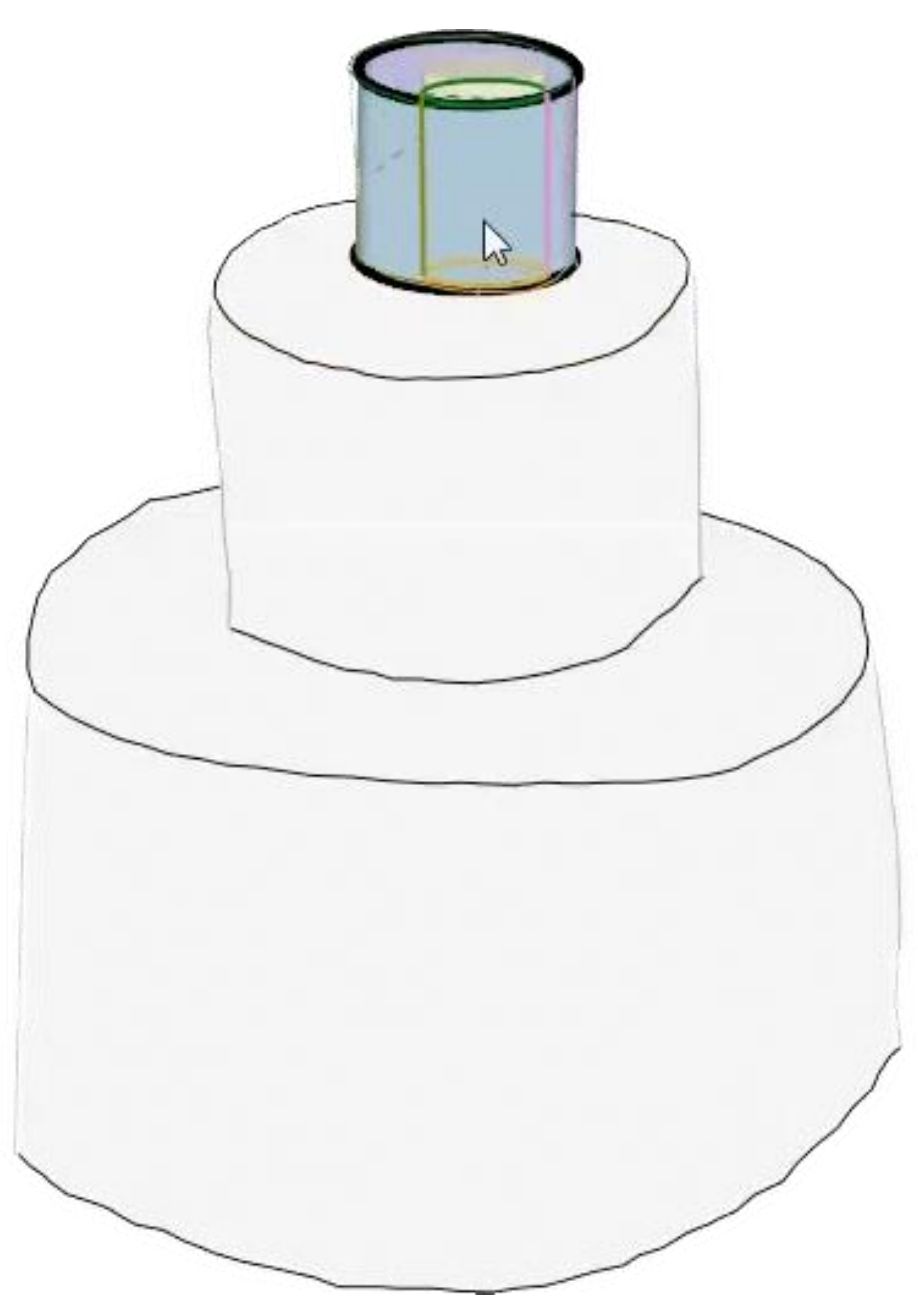


Anatomy of a Drag: Geosemantic Relations

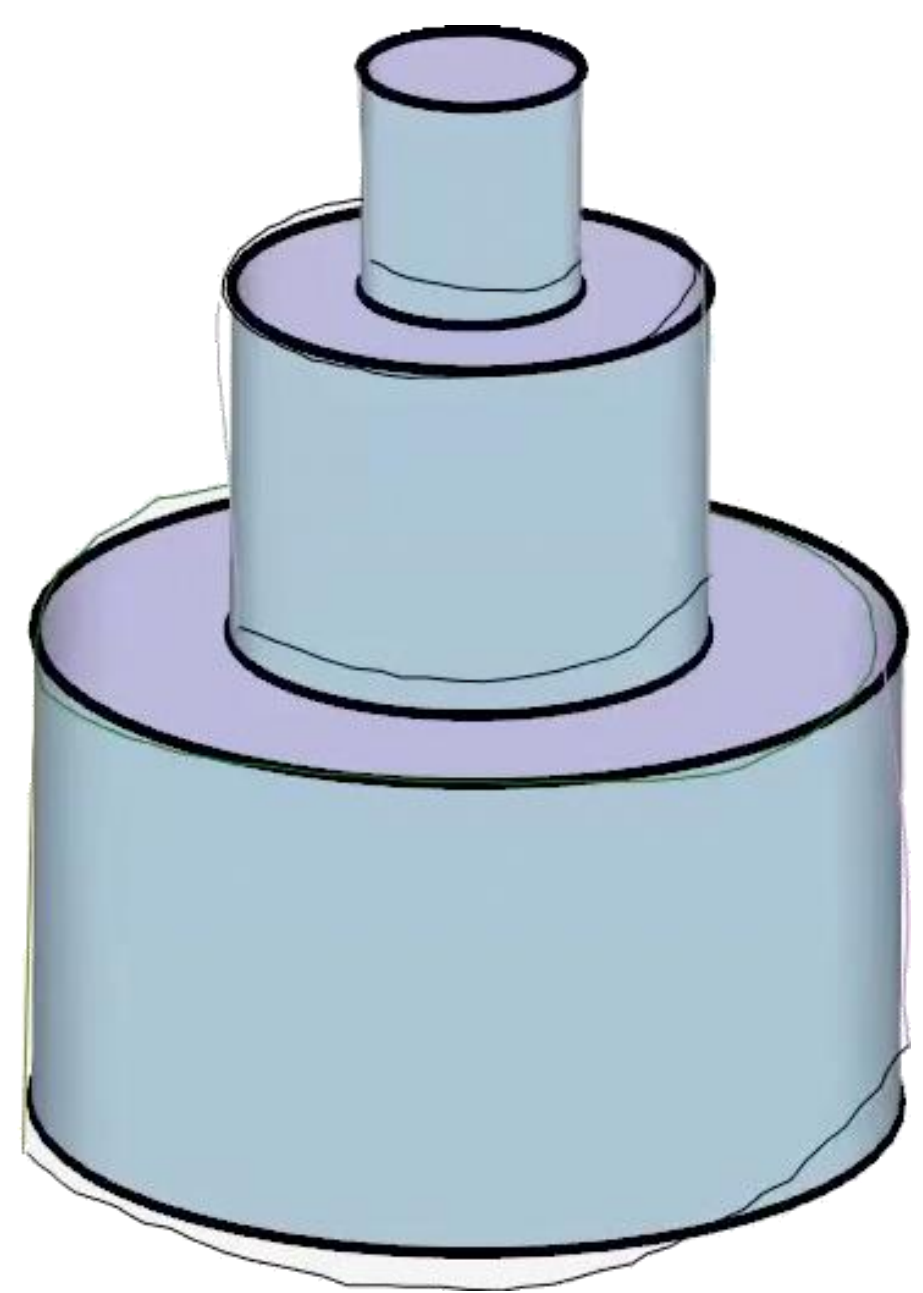

SIGGRAPH

ASIA 2516

45 


\section{Anatomy of a Drag: Geosemantic Relations}

$$
\begin{array}{cll}
\min _{x}: & \sum_{p \in P} \phi_{p}\left(x_{p}\right) & \\
\text { s.t. : } & C_{p}\left(x_{p}\right)=0 & \forall p \in P \\
& \psi_{g}\left(x_{g}\right)=0 \quad \forall g \in G
\end{array}
$$

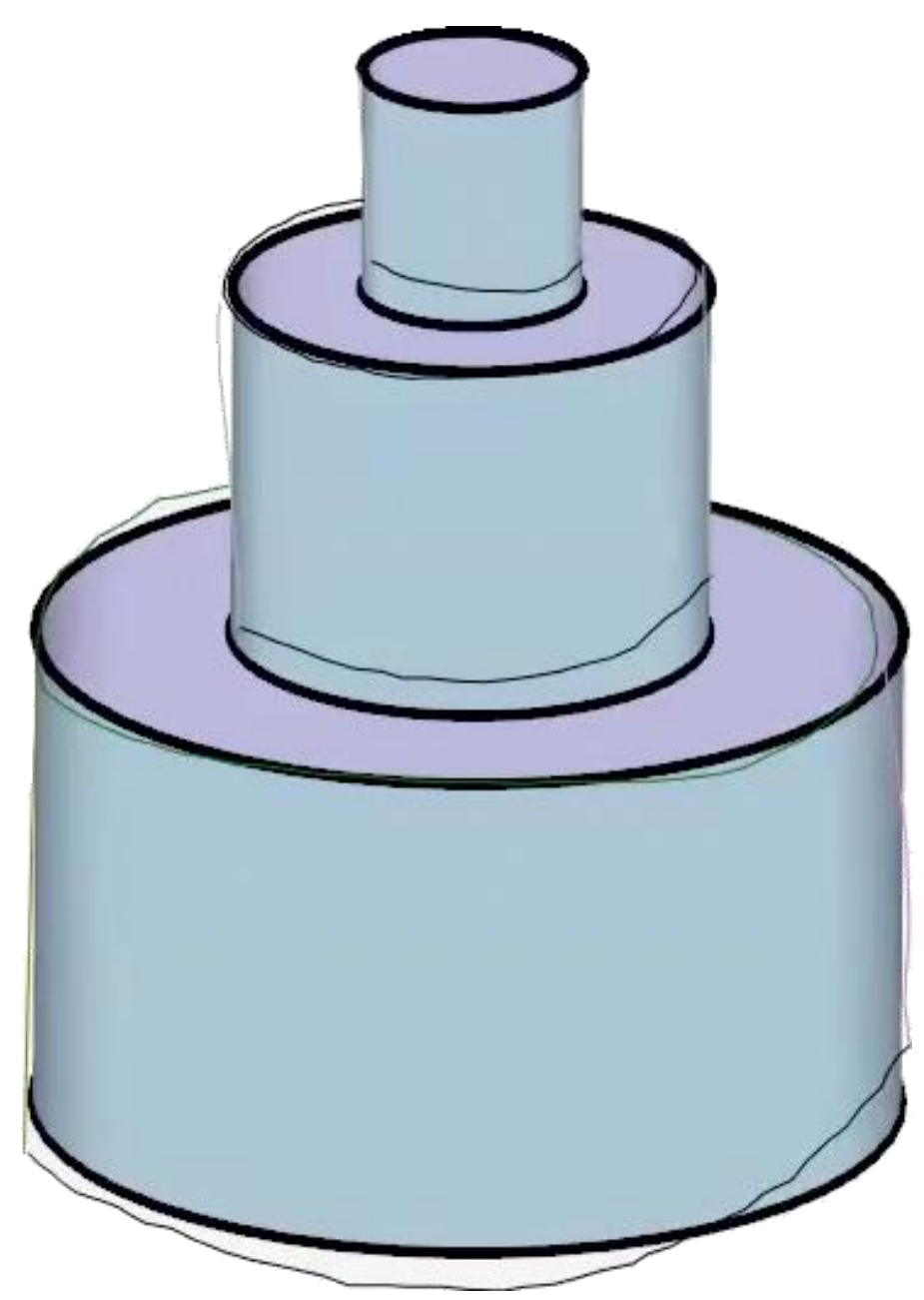




\section{Geosemantic Relations}

Constraints linking two or more feature curves:

- Parallelism

- Orthogonality

- Collinear centers (three or more)

- Concentric

- Coplanar

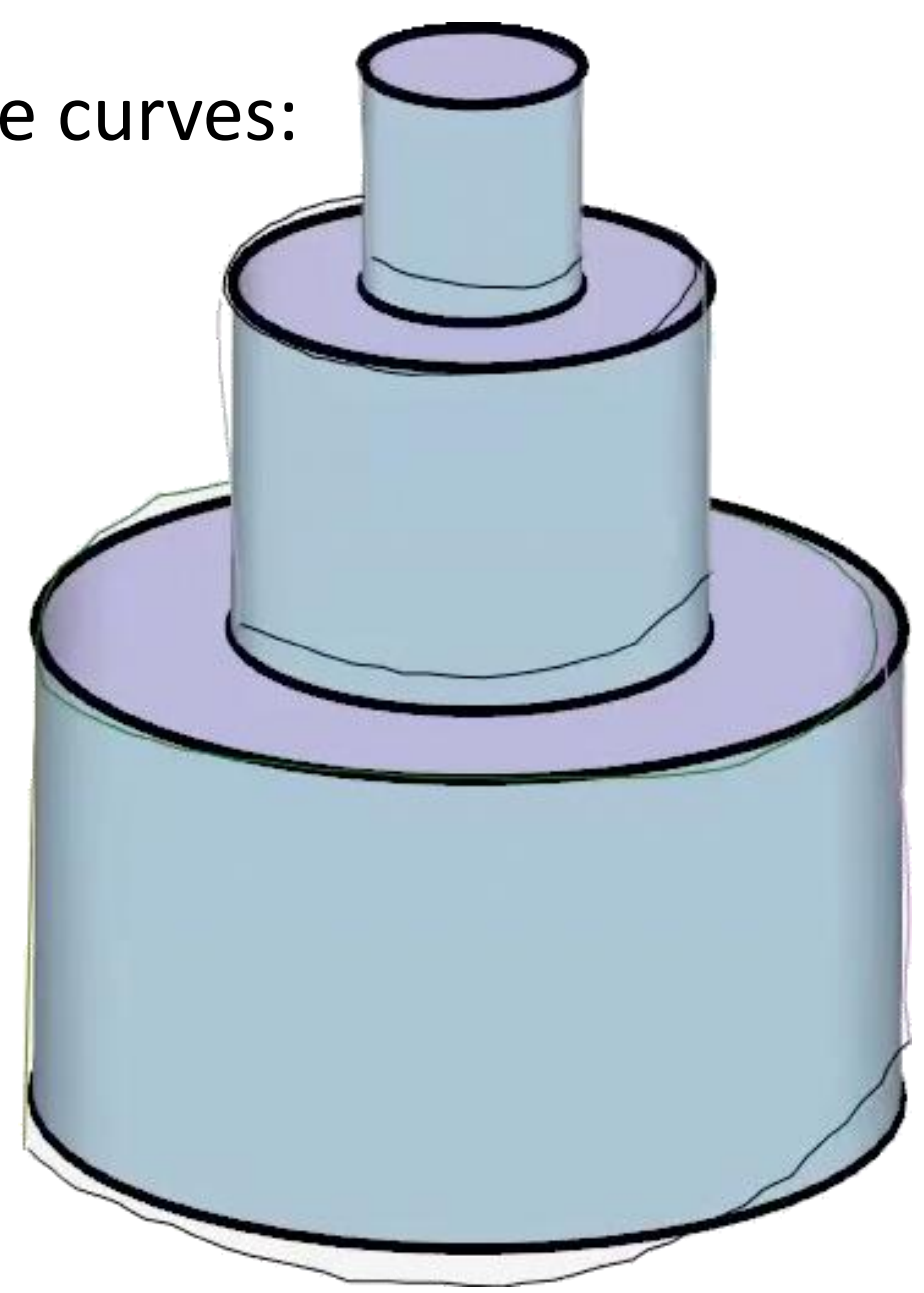




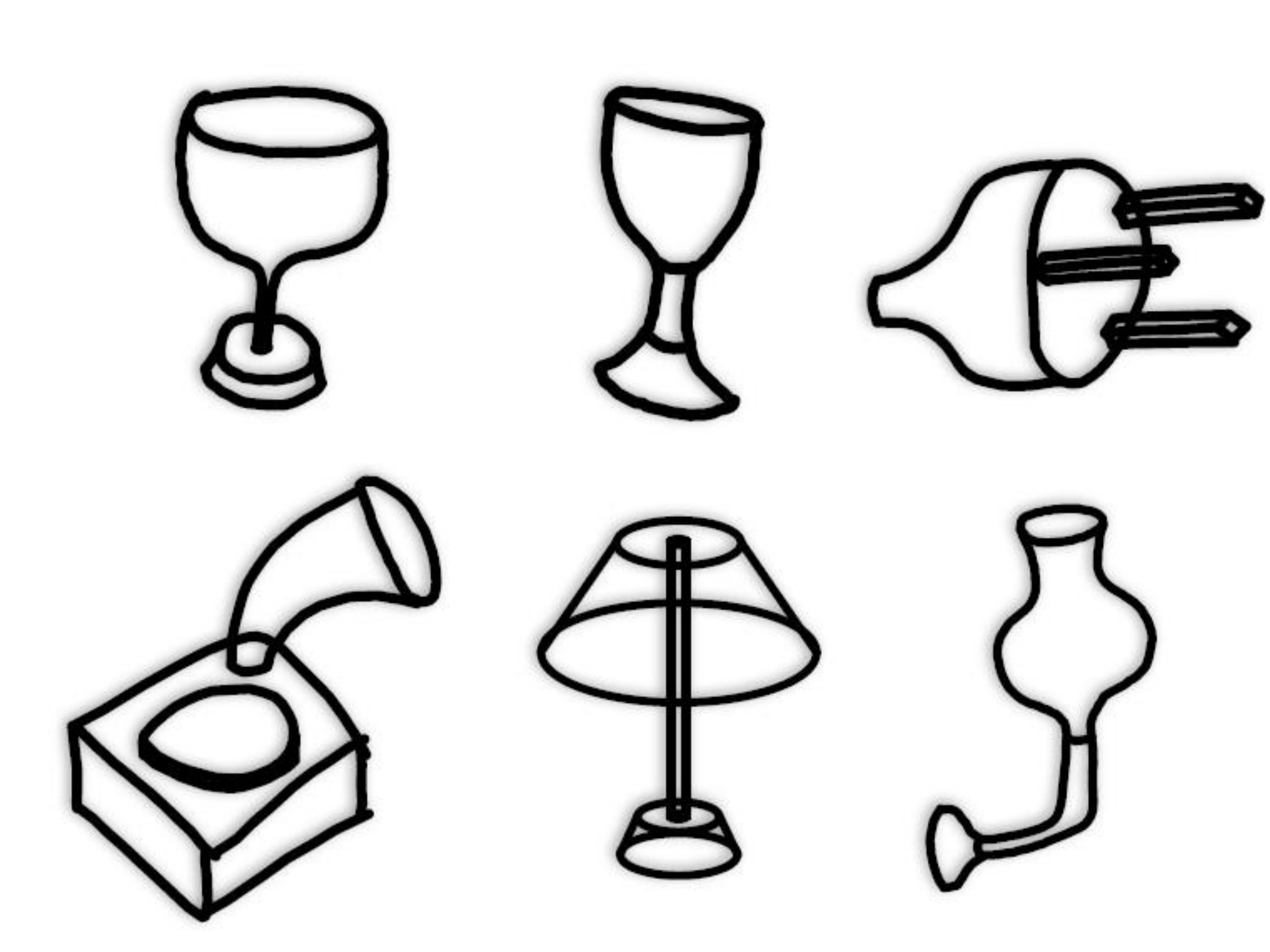



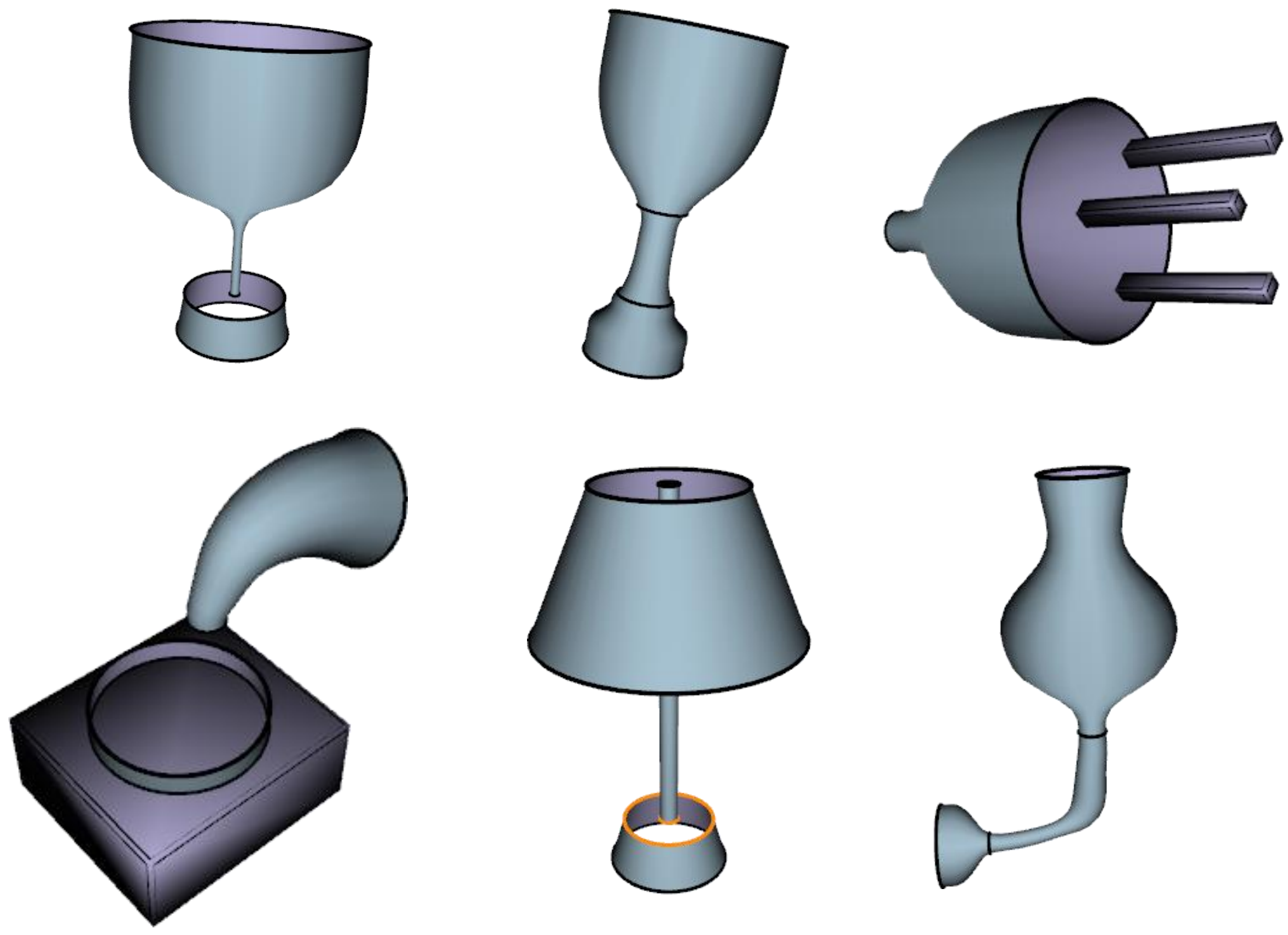


$$
\text { (2ma }
$$



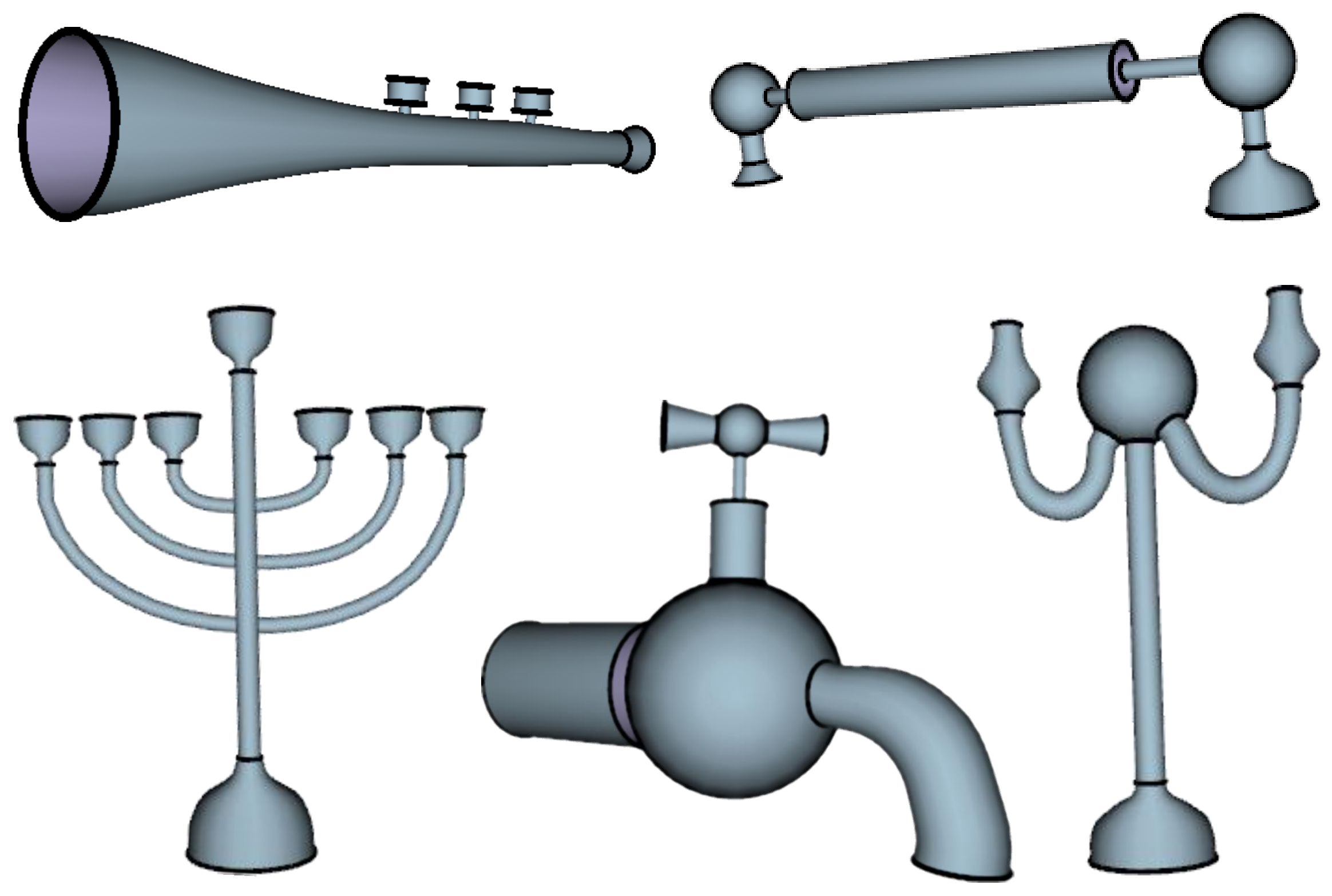


\section{Limitations \& Future Work}

More primitives

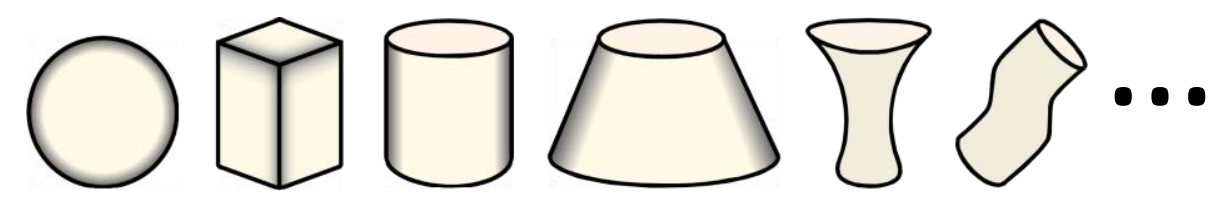

Operate directly on raster sketches

Eliminate sketch curve classification

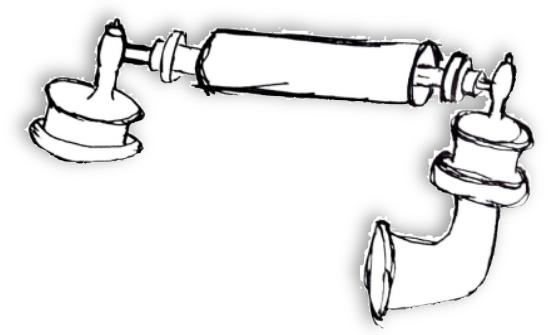

Sketched occlusions

More geosemantic relations

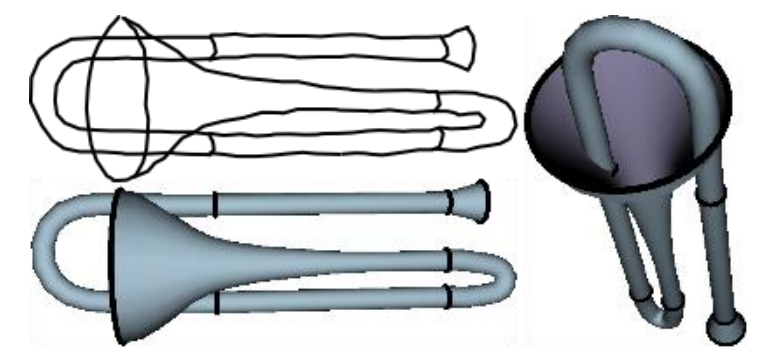




\section{Lifting curve networks into 3D}

- Interactively

- Analytic drawing of 3D scaffolds [Schmidt et al. 2009]

- Automatically

- CrossShade: Shading Concept Sketches Using Cross-Section Curves [Shao et al. 2012]

- True2Form: 3D curve networks from 2D sketches via selective regularization [Xu et al. 2014] 


\section{Analytic drawing of 3D scaffolds [Schmidt et al. 2009]}

- Draw precise scaffold lines by connecting them to 2-point perspective vanishing points

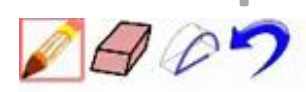




\section{Analytic drawing of 3D scaffolds [Schmidt et al. 2009]}

- The scaffolds make it possible to draw complex curves

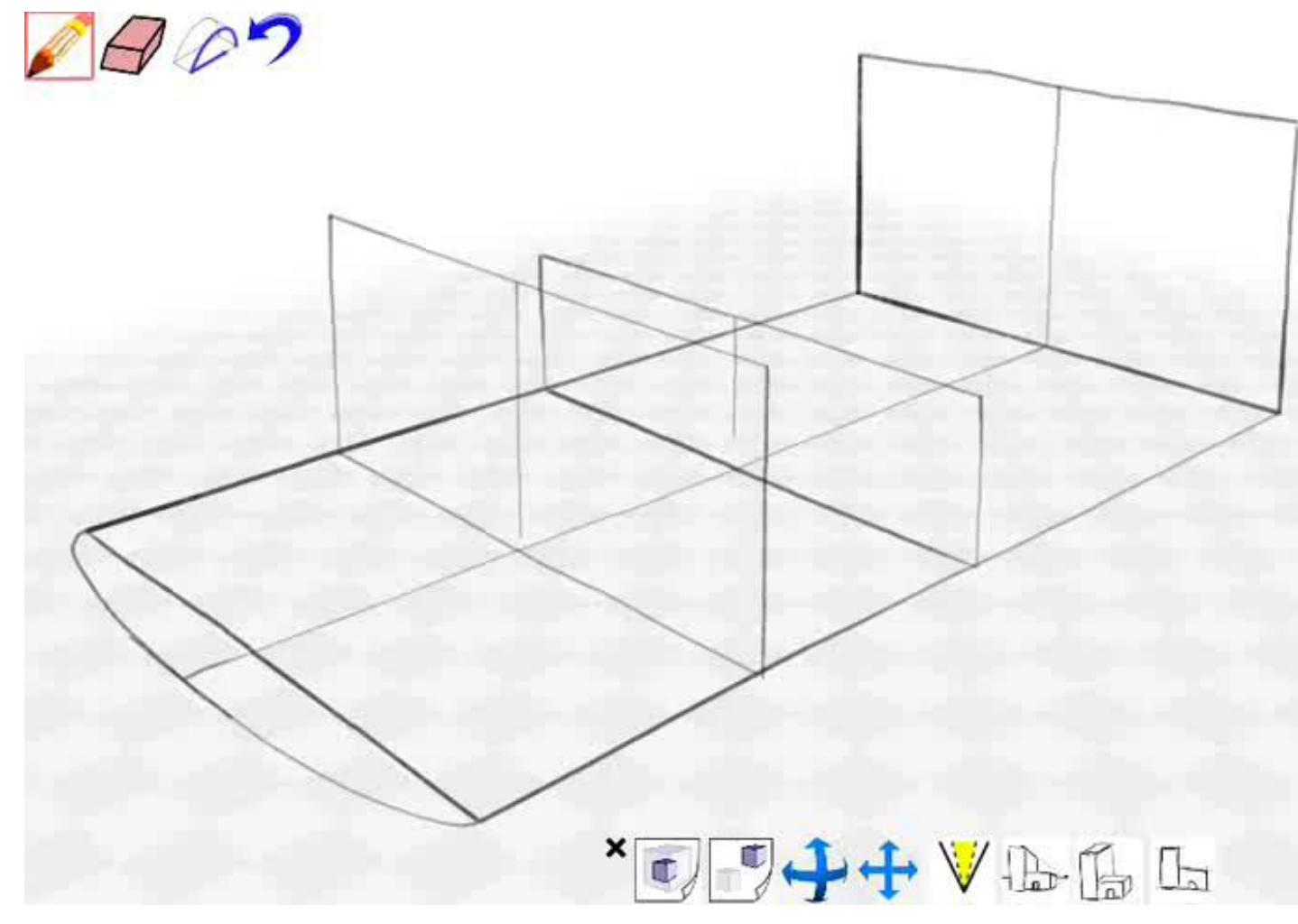




\section{Analytic drawing of 3D scaffolds [Schmidt et al. 2009]}

- ... and complex shapes
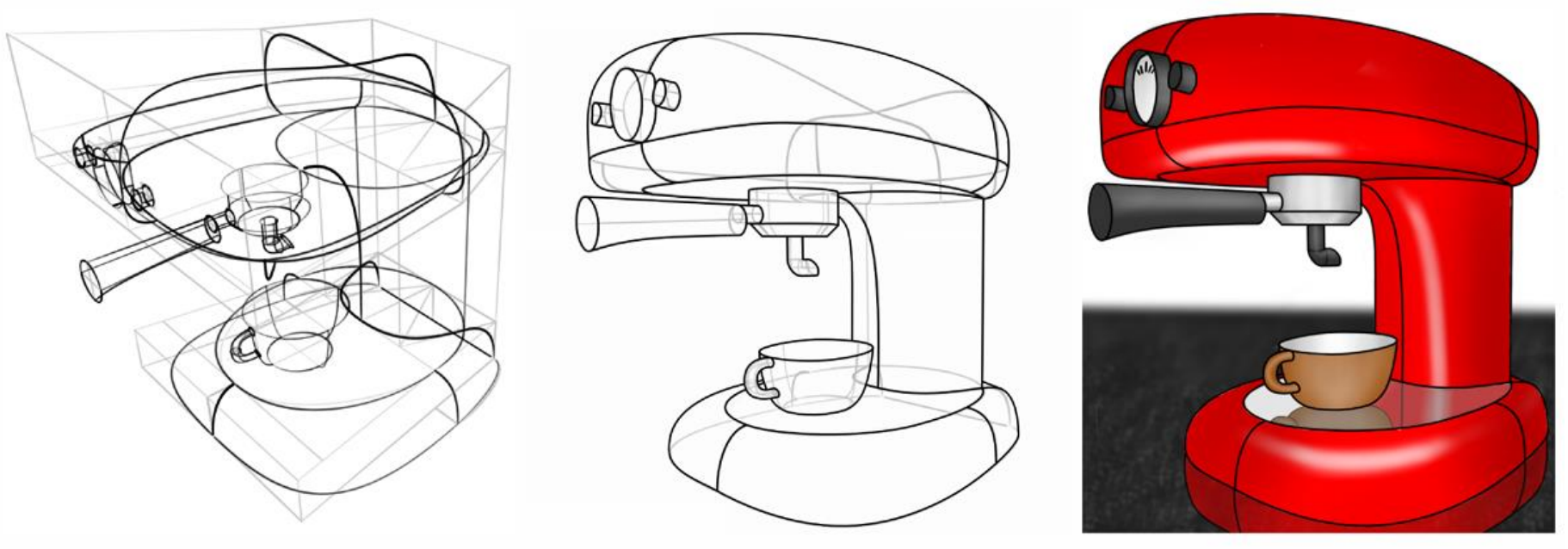
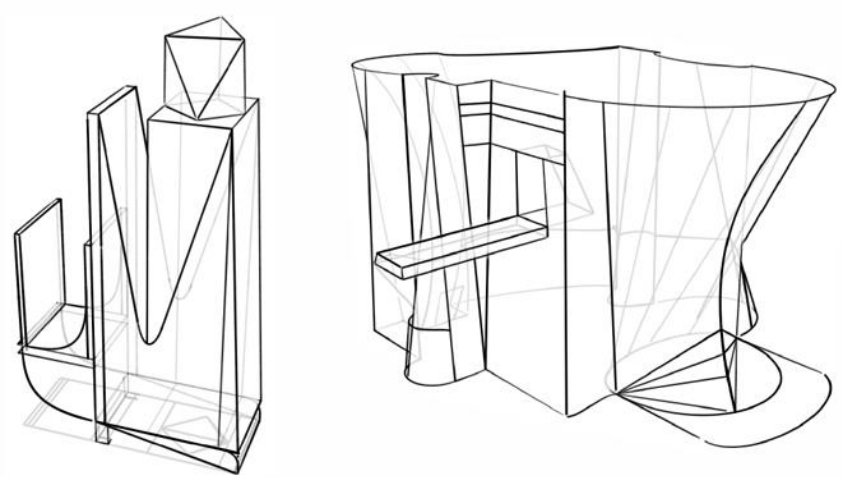

Q\%, SIGGRAPH
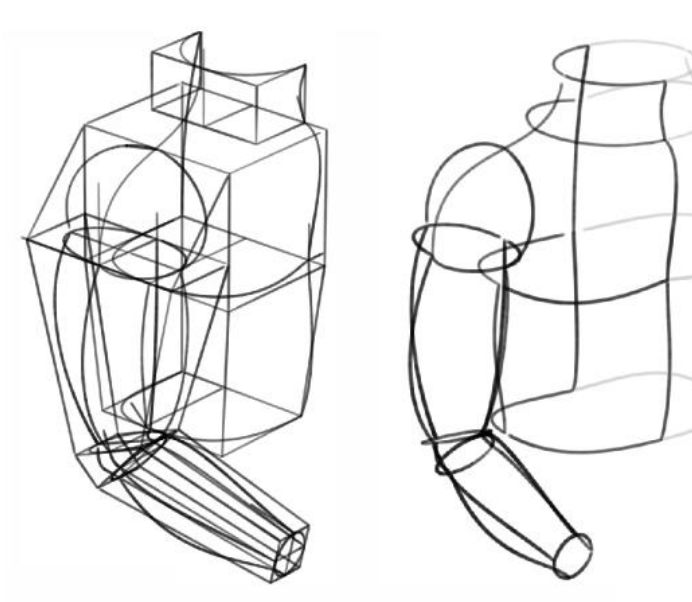
CrossShade: Shading Concept Sketches Using Cross-Section Curves [Shao et al. 2012]

- Leverage properties of designer-drawn cross sections:

- intersect on orthogonal planes

- are aligned with principal curvature (and therefore are orthogonal themselves)

- are geodesics

- intersect with minimal foreshortening 


\section{CrossShade: Shading Concept Sketches Using Cross-Section Curves [Shao et al. 2012]}

- With these cues, we can propagate normals everywhere:

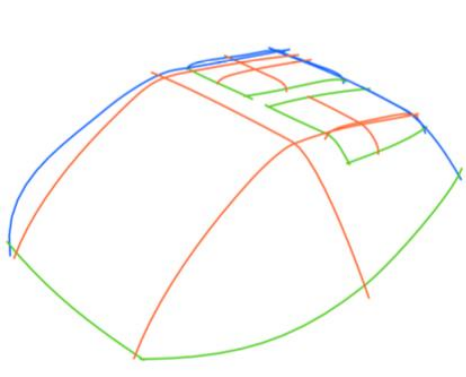

(a) Input curves

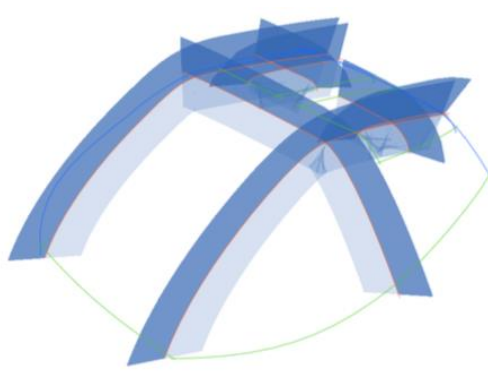

(b) Estimated curve planes

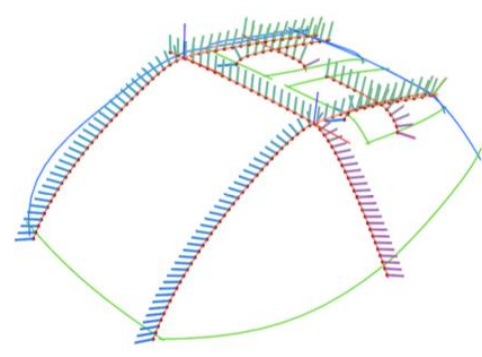

(c) Normals along curves

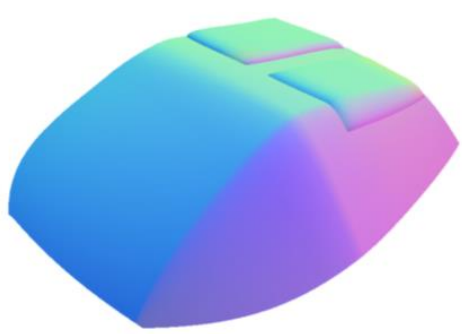

(d) Normals over the sketch

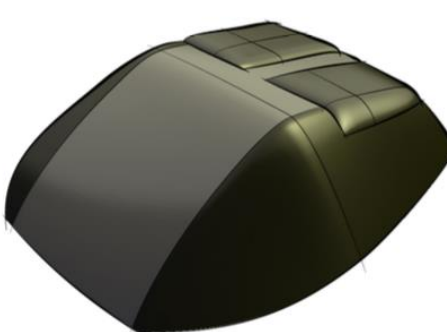

(e) Resulting shading 
CrossShade: Shading Concept Sketches Using Cross-Section Curves [Shao et al. 2012]
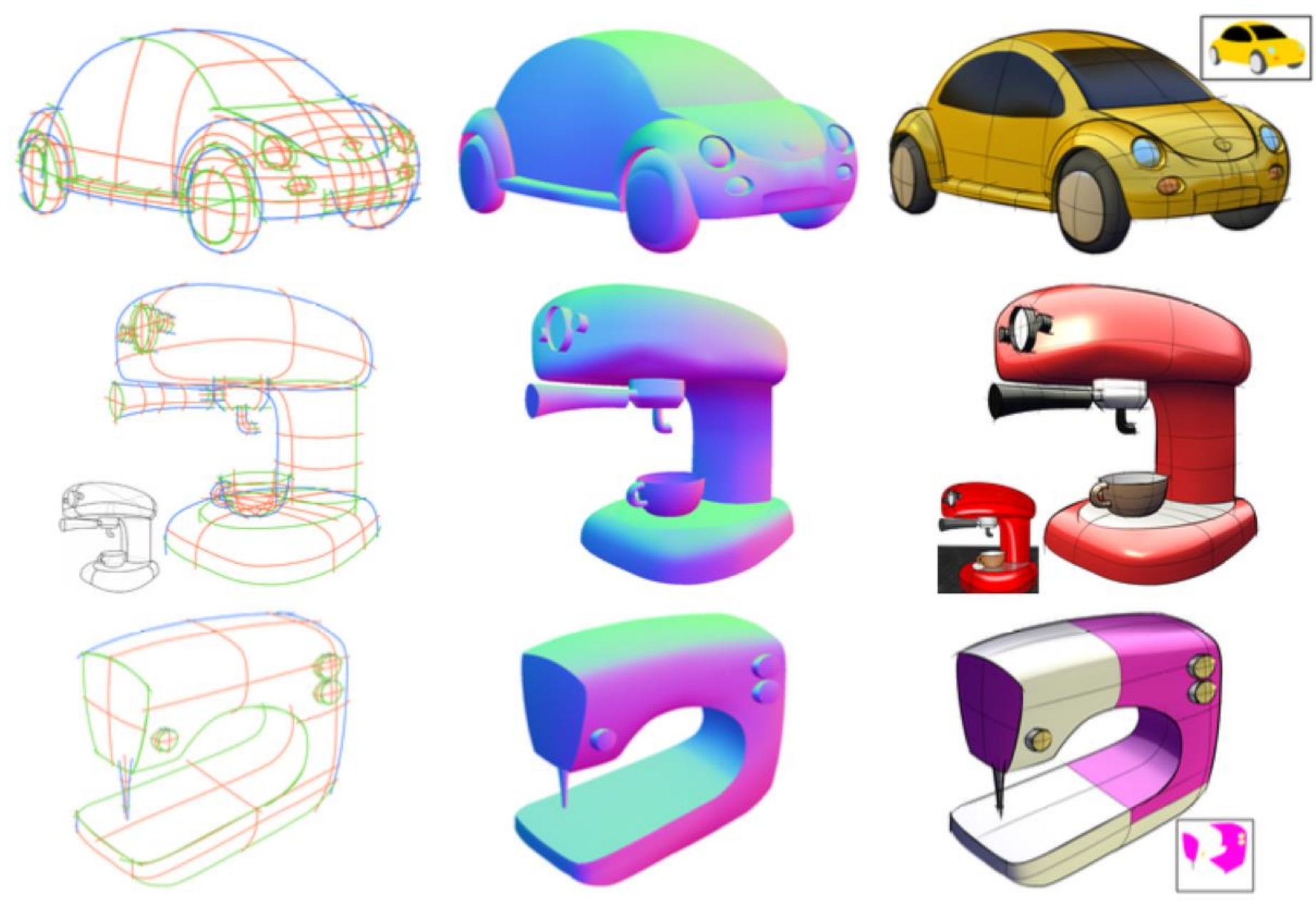

(a) Input curves

(b) Normals

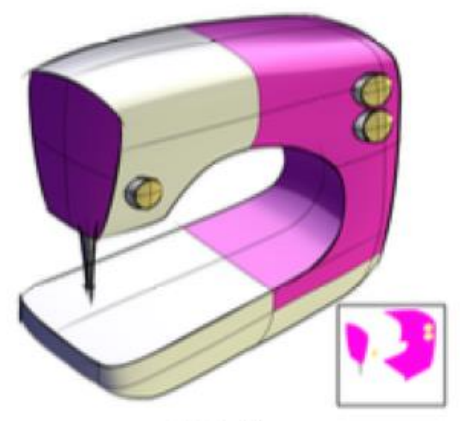

(c) Shading

Q?, SIGGRAPH 
True2Form: 3D curve networks from 2D sketches via selective regularization [Xu et al. 2014]

- Given 2D curves, we can selectively apply the constraints in an optimization to get 3D curves

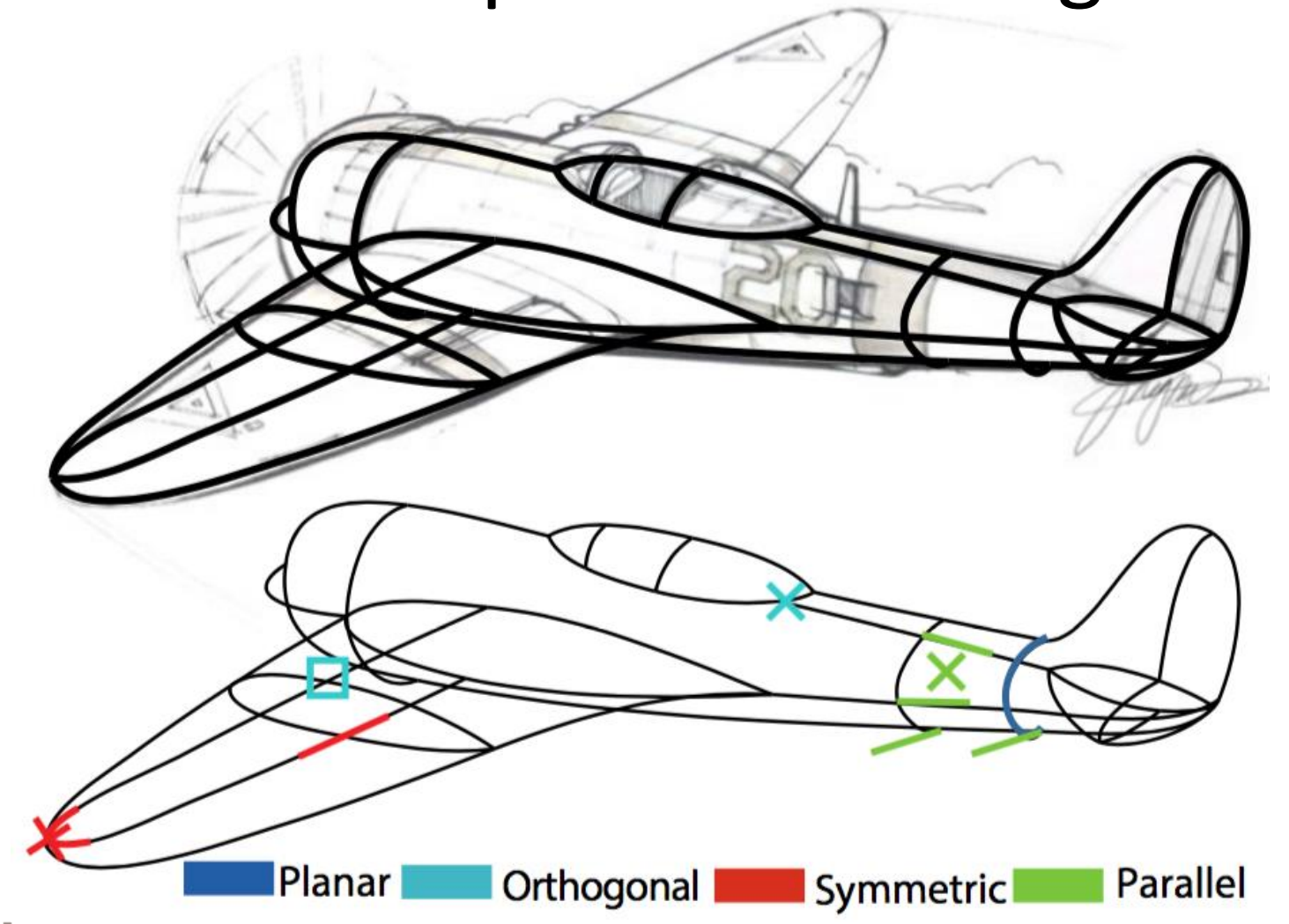

$$
\text { Planar Orthogonal - Symmetric Parallel }
$$


True2Form: 3D curve networks from 2D sketches via selective regularization [Xu et al. 2014]

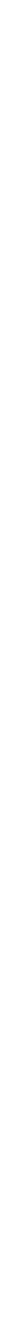

QQ, SIGGRAPH 


\section{True2Form: 3D curve networks from 2D sketches via selective regularization [Xu et al. 2014]}

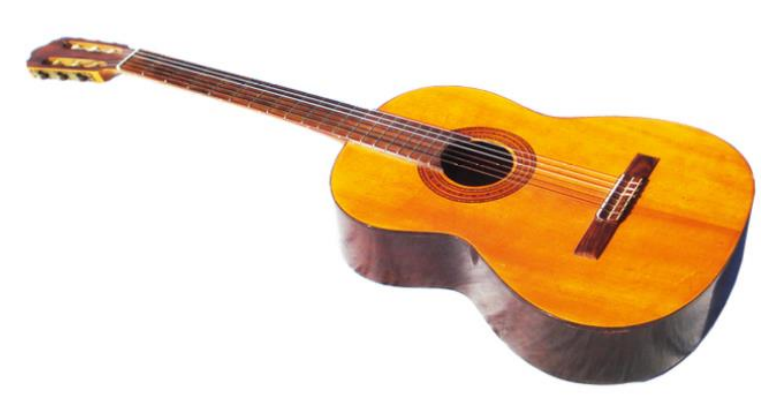

Inspiration

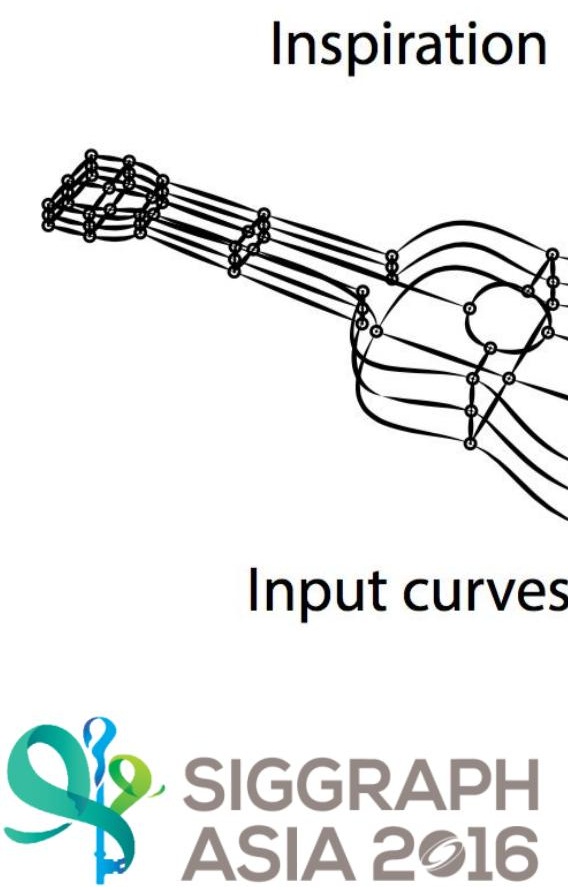

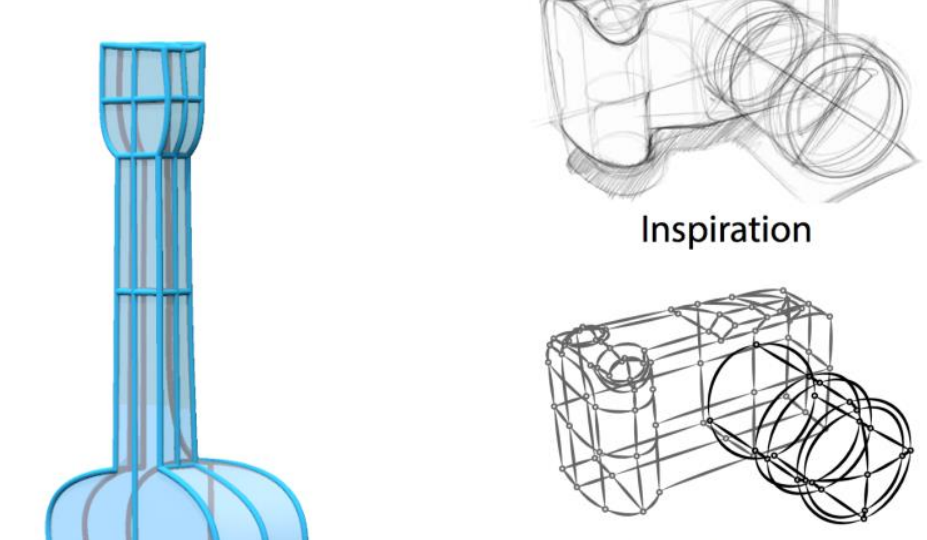

Input curves

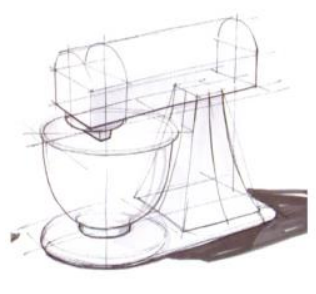

Inspiration

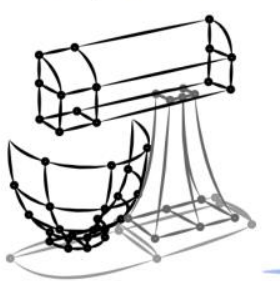

Input curves

3D Reconstruction
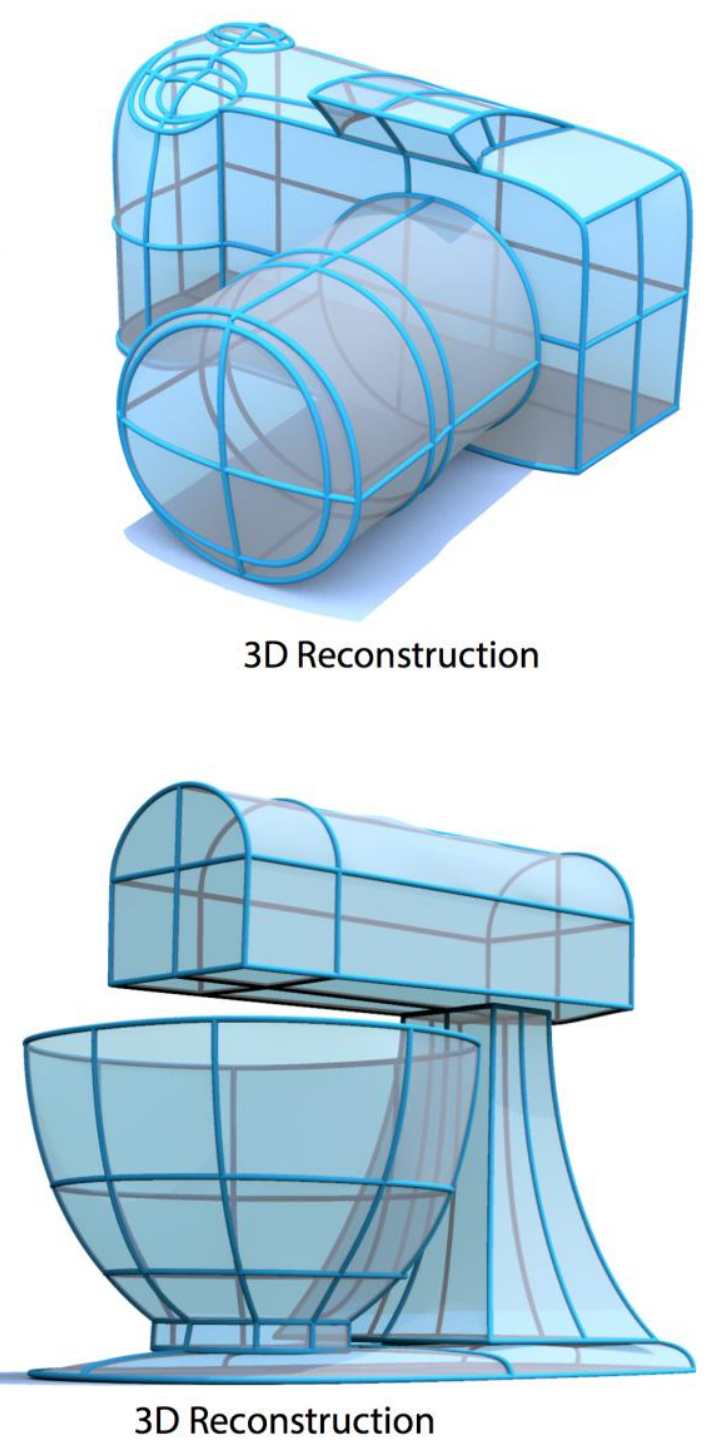


\section{Takeaways}

- Make "intractable" problems tractable with perceptually grounded assumptions or by asking the user to help.

- Don't ask the user for too much. Separate that which is easy for a human and challenging for a computer.

- Consult artistic practice and perceptual psychology for inspiration. 
Single-view sketch-based modeling of 3D curves and surfaces

Part II

Frederic Cordier 


\section{Free-Form Sketching of Self-Occluding Objects}
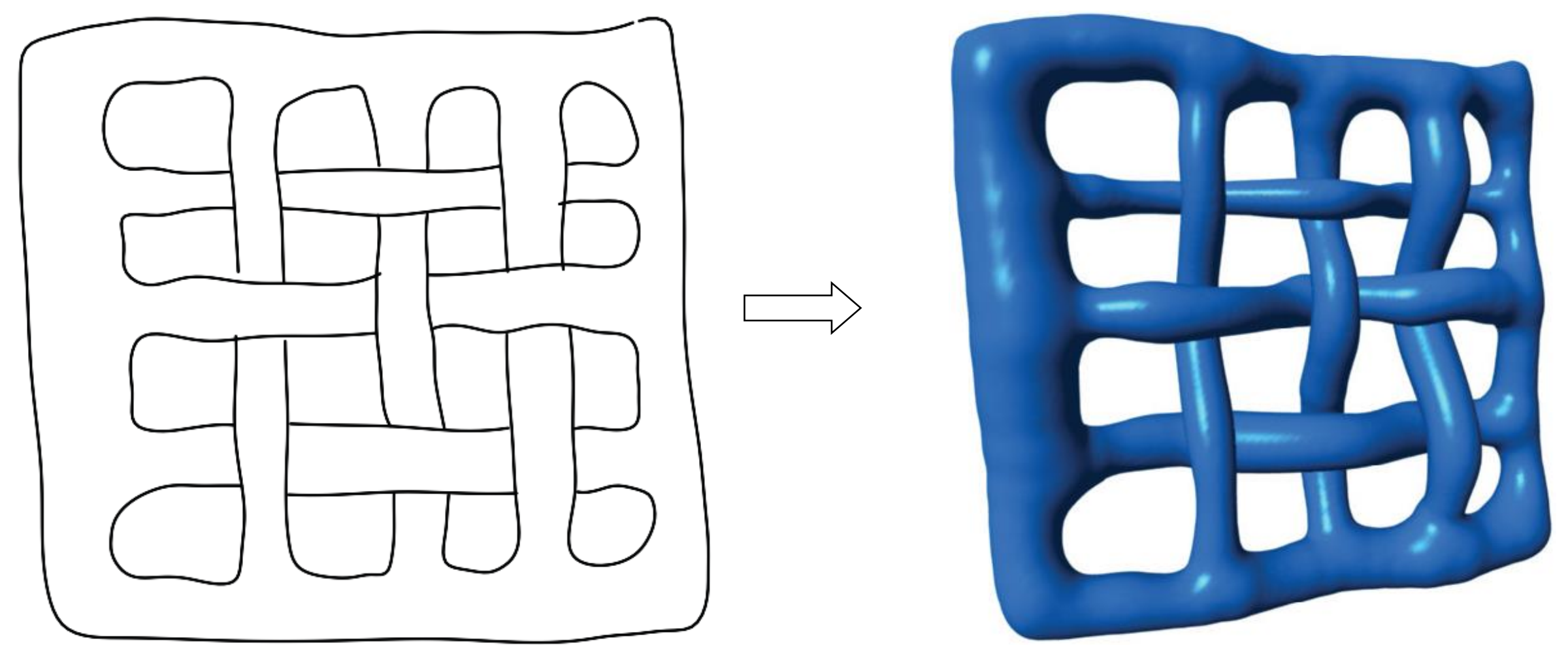

Frederic Cordier, Hyewon Seo: Free-Form Sketching of Self-Occluding Objects. IEEE Computer Graphics and Applications 27(1): 50-59 (2007) 


\section{Sketching of Mirror-Symmetric Shapes}

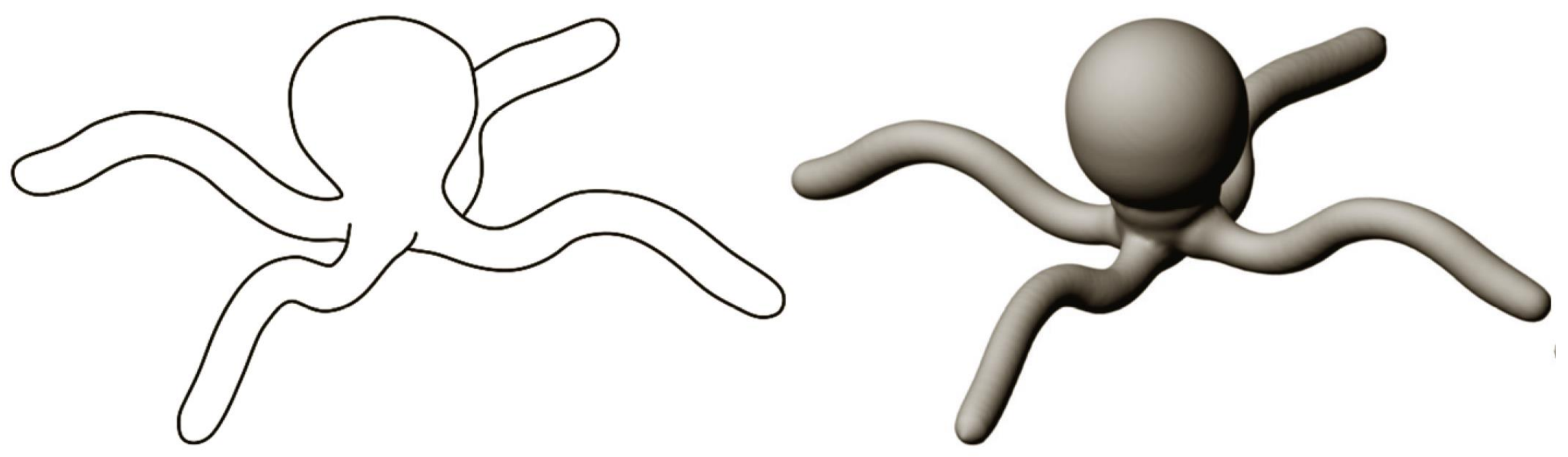

Frederic Cordier, Hyewon Seo, Jinho Park, Jun-yong Noh: Sketching of Mirror-Symmetric Shapes. IEEE Trans. Vis. Comput. Graph. 17(11): 1650-1662 (2011) 


\section{Reconstruction of helices from their orthogonal projection}
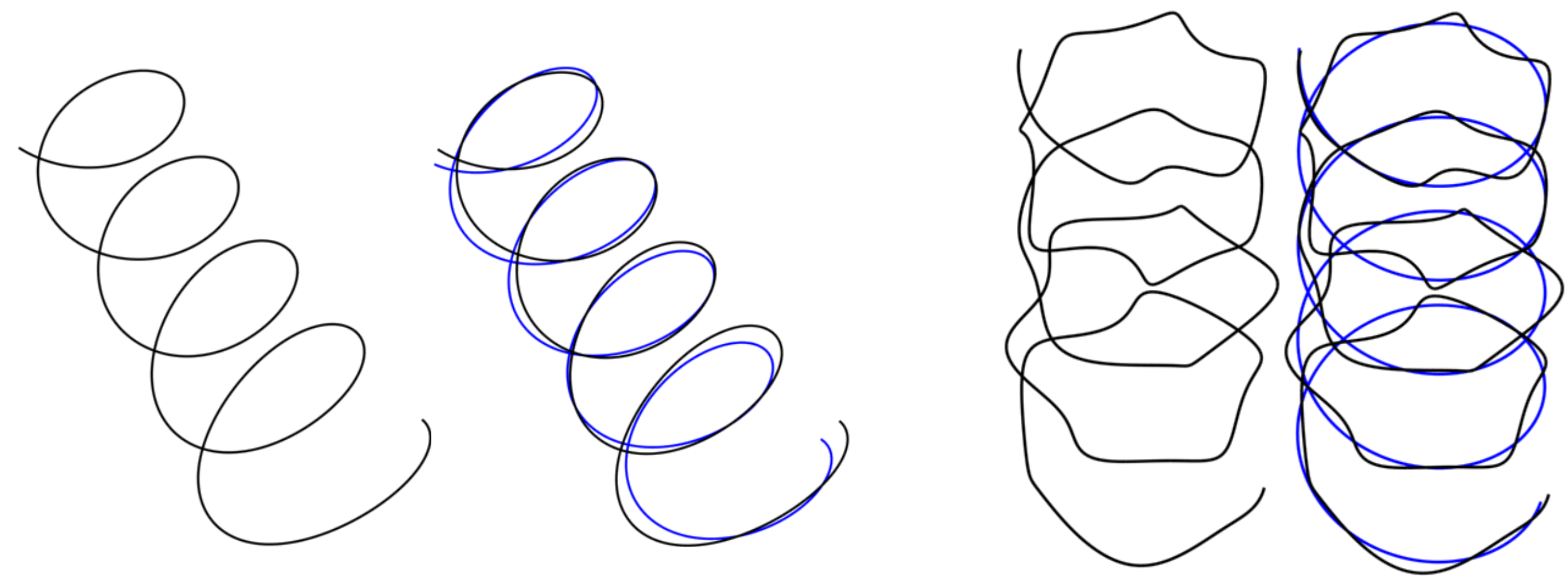

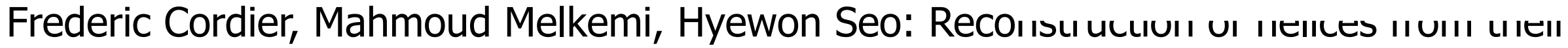
orthogonal projection. Computer Aided Geometric Design, Volume 46, August 2016, Pages $1-15$ 
Reconstruction of helices from their orthogonal projection

- Reconstruction of curves of constant curvature
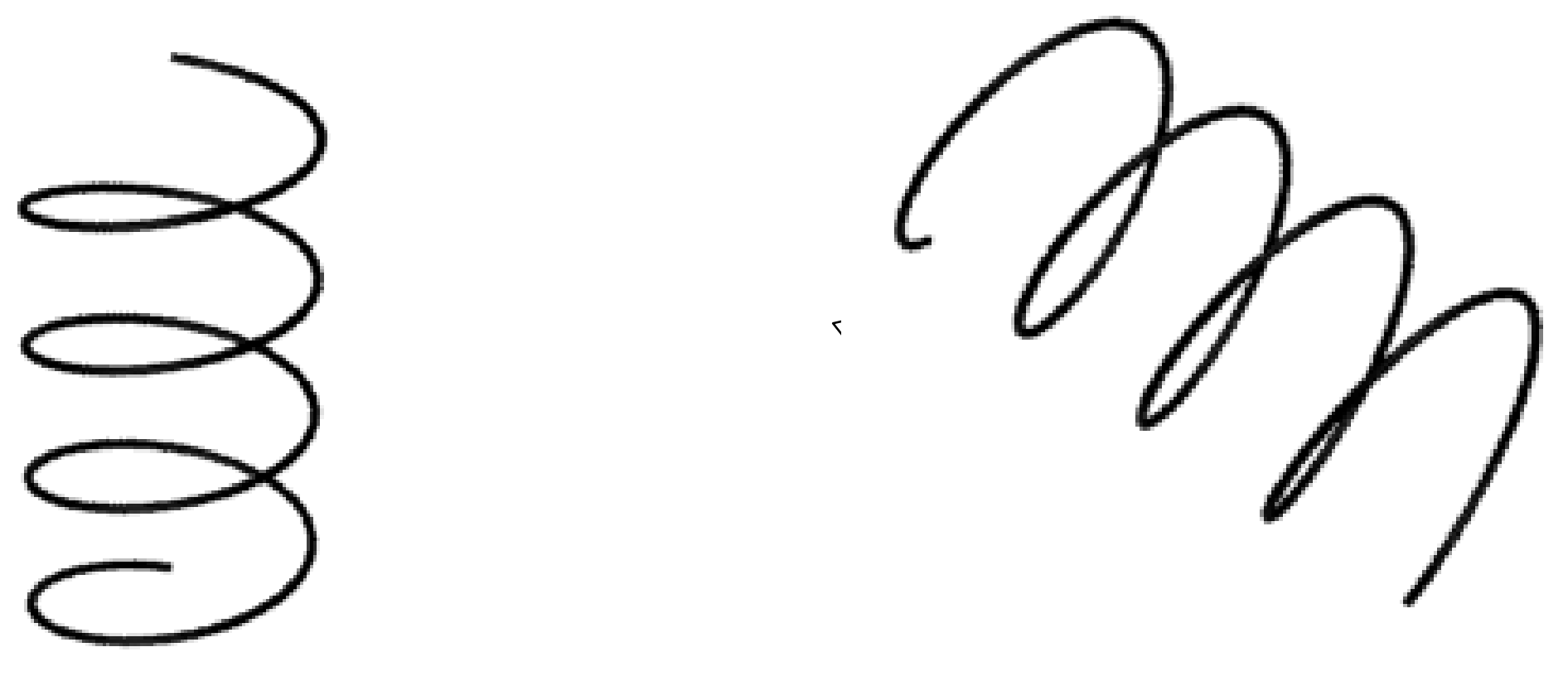


\section{Reconstruction of helices from their orthogonal projection}

Parametric equation of a helix of radius $r$ and pitch $p$ :

$$
H(t)=\left[\begin{array}{c}
r \cos (t) \\
p t \\
r \sin (t)
\end{array}\right]
$$

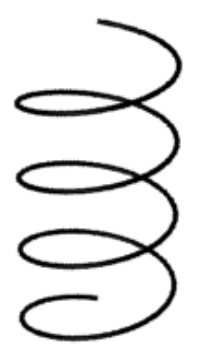

Computing $r, p$ and the projection matrix requires nonlinear optimization!

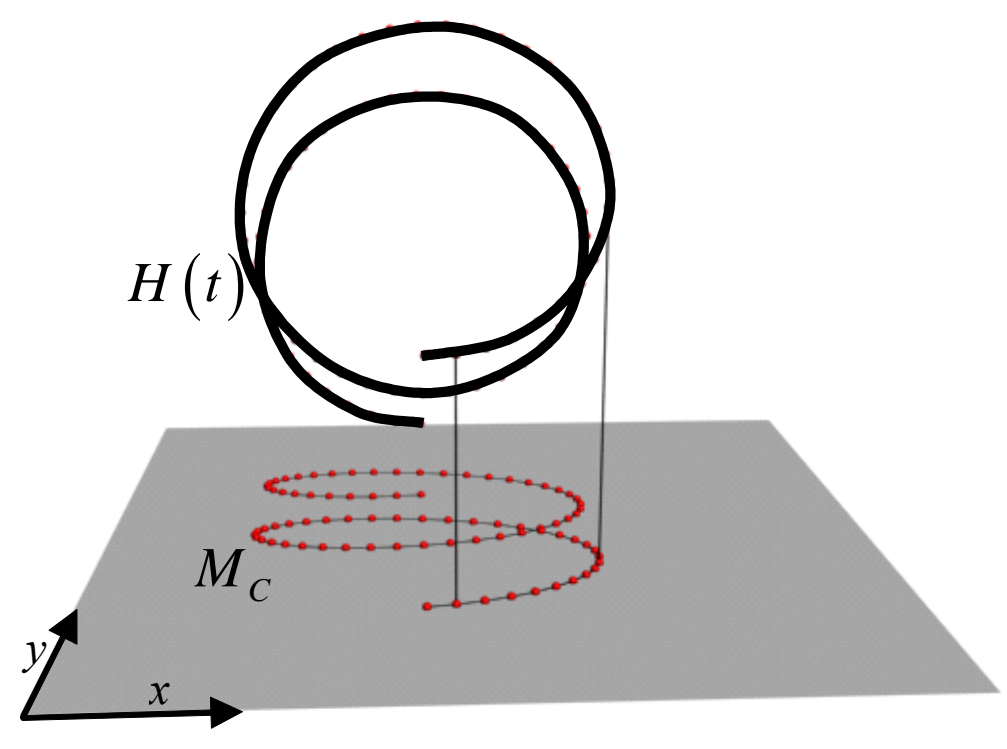




\section{Reconstruction of helices from their orthogonal projection}

Sampling of the helix

Compute the affine transformation $L$

Compute the rotation matrix and the helix

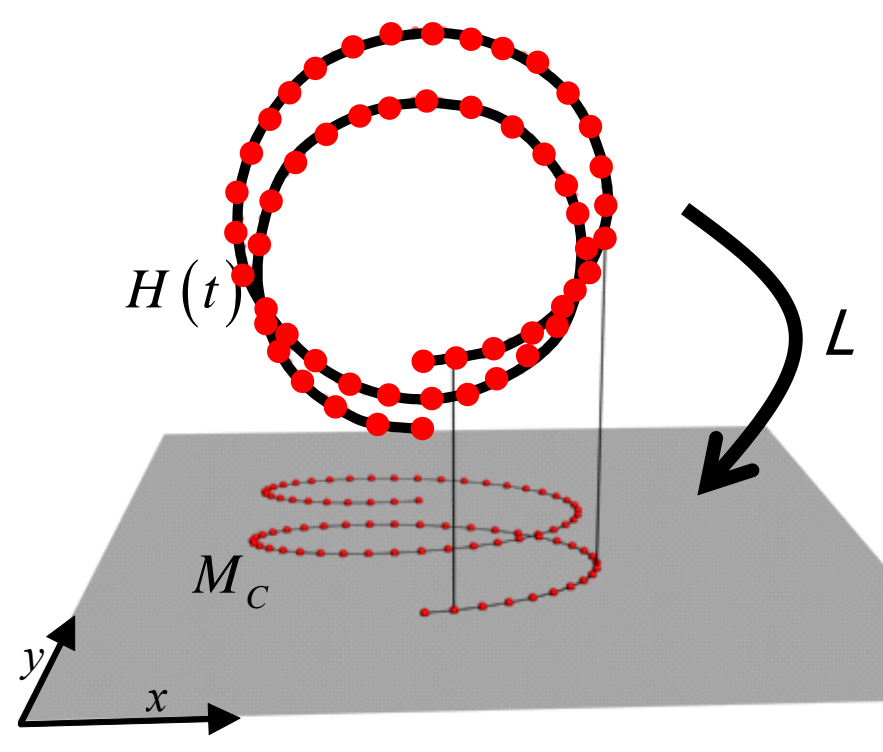
parameters 


\section{Reconstruction of helices from their orthogonal projection}

The key idea:
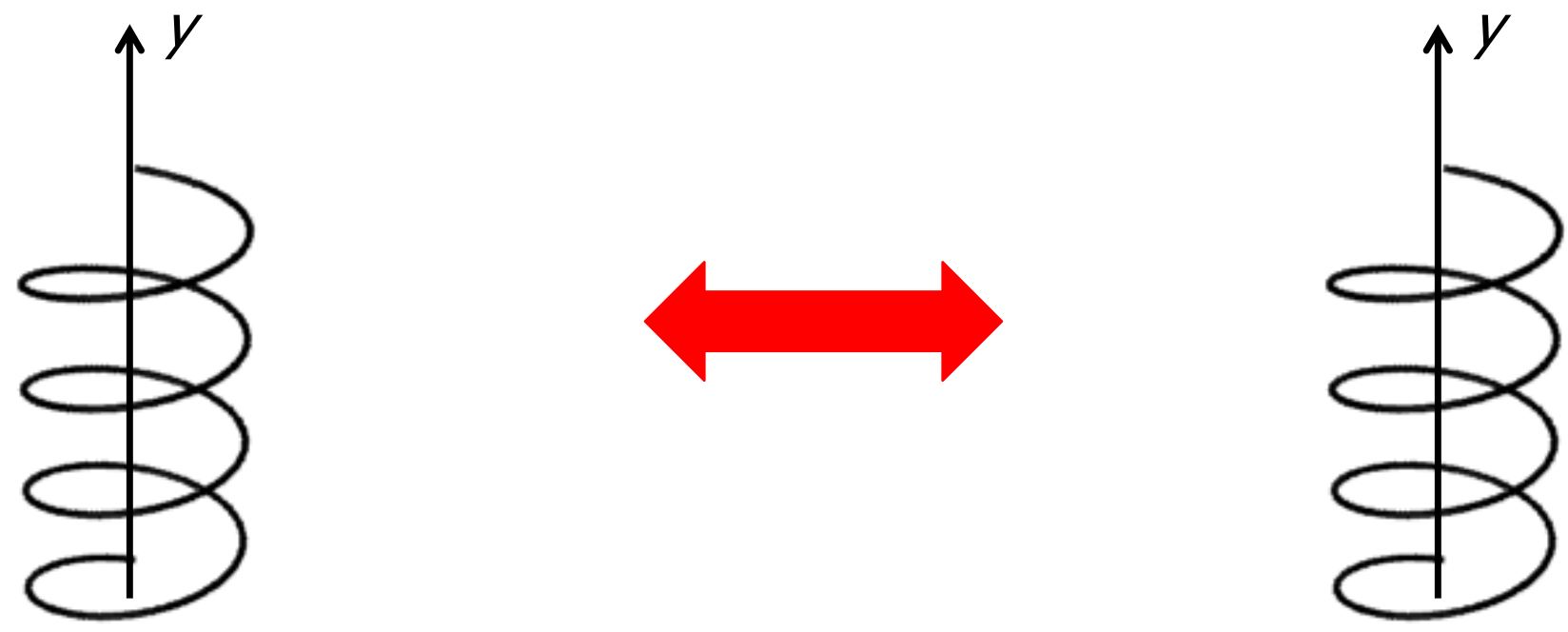

Pitch equal to 1

Scale along $y$ equal to 2

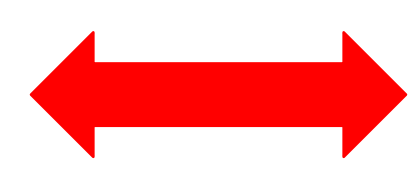

Pitch equal to 2

Scale along $y$ equal to 1 


\section{Reconstruction of helices from their orthogonal projection}

\section{Sampling of the helix}

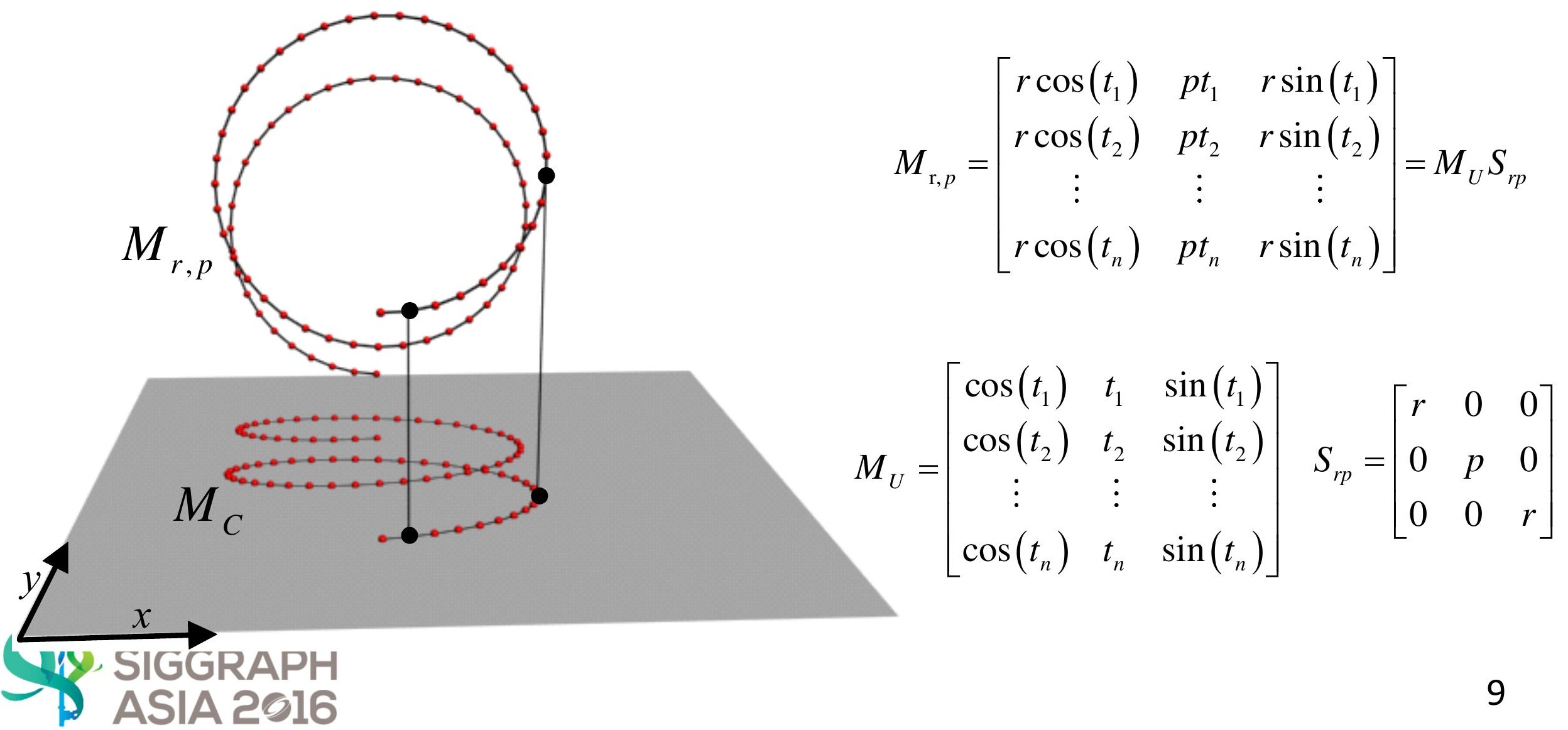




\section{Reconstruction of helices from their orthogonal projection}

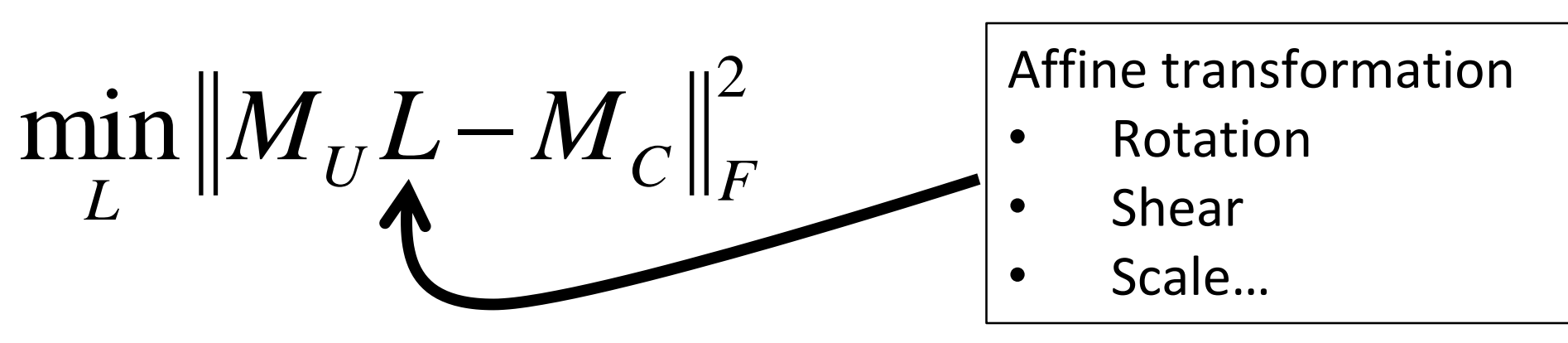

$$
\left\|M_{U} L-M_{C}\right\|_{F}^{2}=\|\left(M_{U} S_{r p}\right) \underbrace{\left(S_{r p}^{-1} L\right)}-M_{C}\|_{F}^{2}
$$

Should be close to orthonormal (i.e. rotation matrix)

Key idea: changing the scaling transformation of the helix is equivalent to changing its radius and pitch 


\section{Reconstruction of helices from their orthogonal projection}

$\left(S_{r p}^{-1} L\right)$ is a matrix with orthonormal columns if

$$
\left(S_{r p}^{-1} L\right)^{T}\left(S_{r p}^{-1} L\right)=I
$$

We solve

$$
\min _{r, p}\left\|\left(S_{r p}^{-1} L\right)^{T}\left(S_{r p}^{-1} L\right)-I\right\|_{F}^{2}
$$




\section{Reconstruction of helices from their orthogonal projection}

Advantages:

- Method that requires solving simple linear systems

- Much faster than using non-linear optimization

- Provides an approximate solution which is very close to the exact solution 
Reconstruction of helices from their orthogonal projection

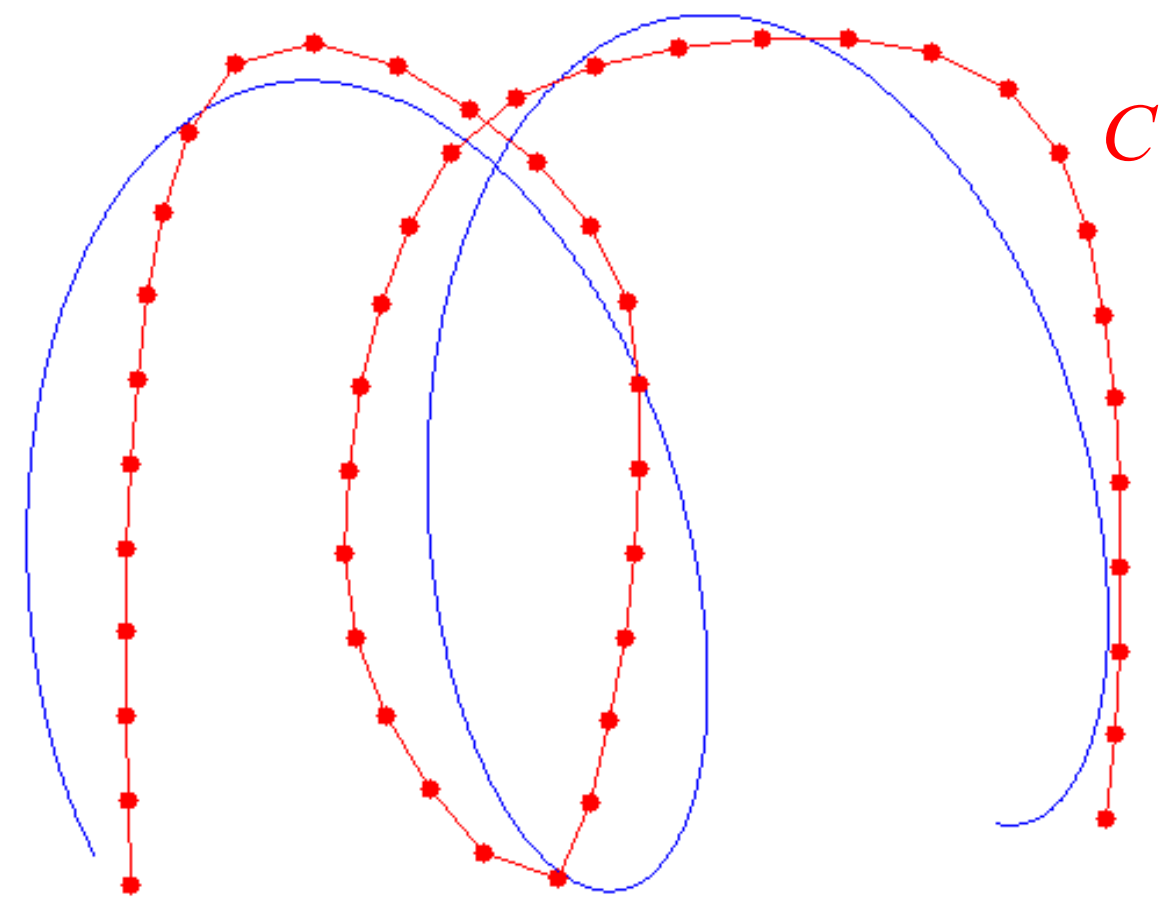




\section{Reconstruction of helices from their orthogonal projection}

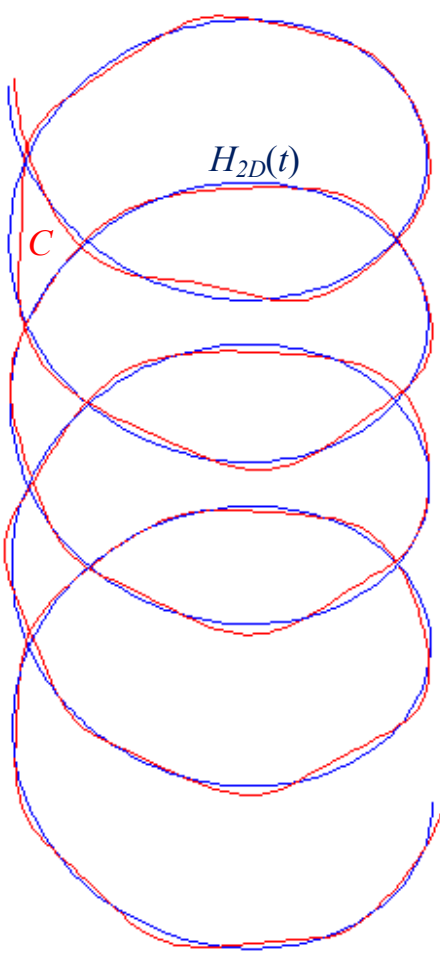

Magnitude of the noise: 0.5 $r=4.0076$ $p=0.9879$ $\alpha=14.1372$ Fitting error: $0.2586 \%$

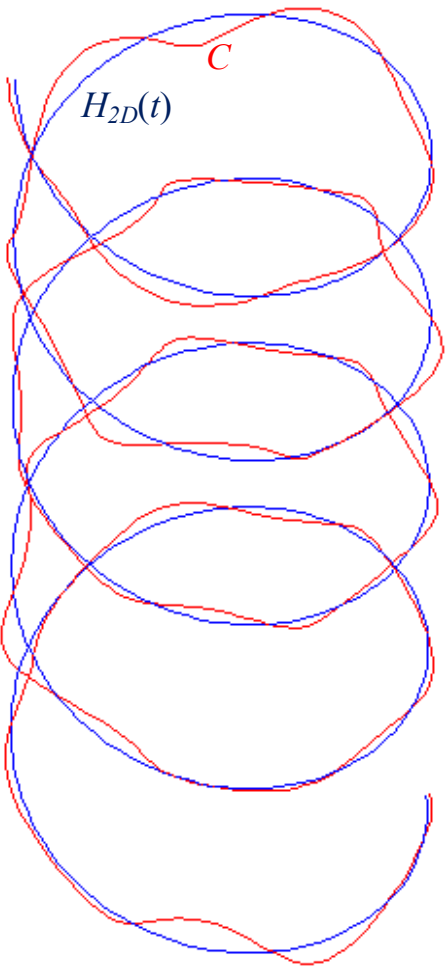

Magnitude of the noise: 1.0 $r=4.0055$
$p=0.9992$
$\alpha=14.1372$

Fitting error: $0.6561 \%$

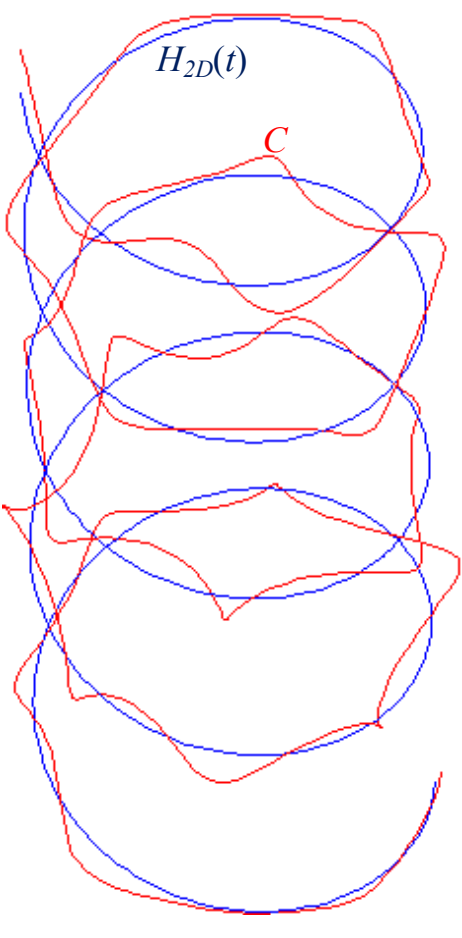

Magnitude of the noise: 1.5 $r=3.9417$
$p=0.9603$
$\alpha=14.0744$

Fitting error: $0.7880 \%$

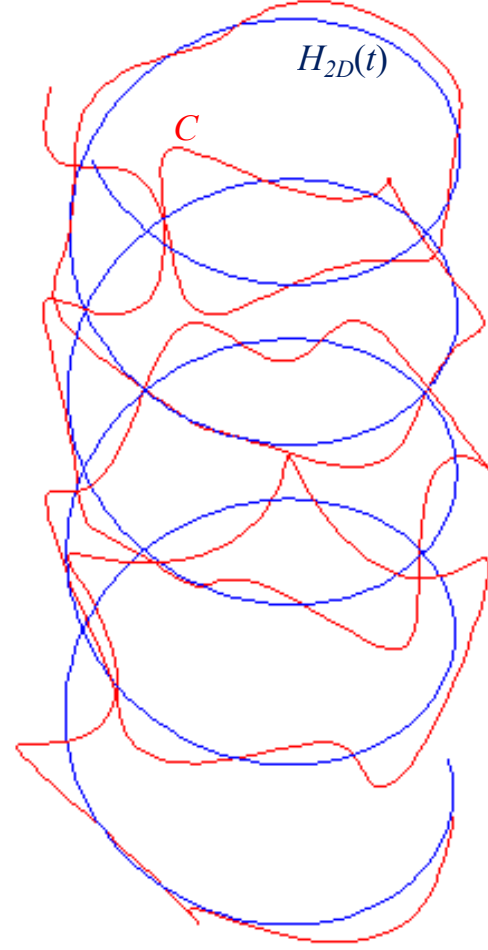

Magnitude of the noise: 2.0 $r=3.7610$
$p=1.0684$
$\alpha=14.0115$

Fitting error: $1.0579 \%$

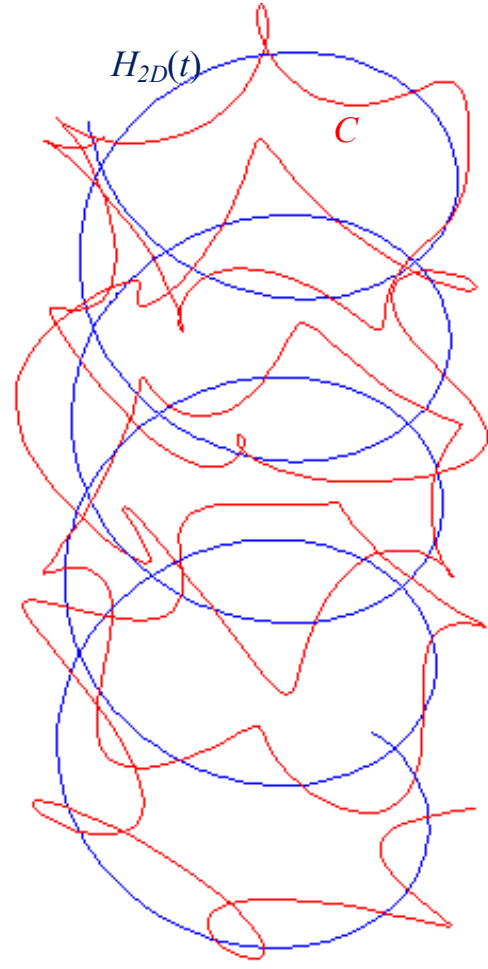

Magnitude of the noise: 3.0

$$
\begin{aligned}
& r=3.8014 \\
& p=1.0714 \\
& \alpha=14.4514
\end{aligned}
$$

Fitting error: $2.6036 \%$ 


\section{Inferring mirror symmetric 3D curves from sketches}

- Input: the 2D sketch of a mirror-symmetric 3D shape

- Output: a set of 3D curves such that their orthogonal projection matches the input sketch

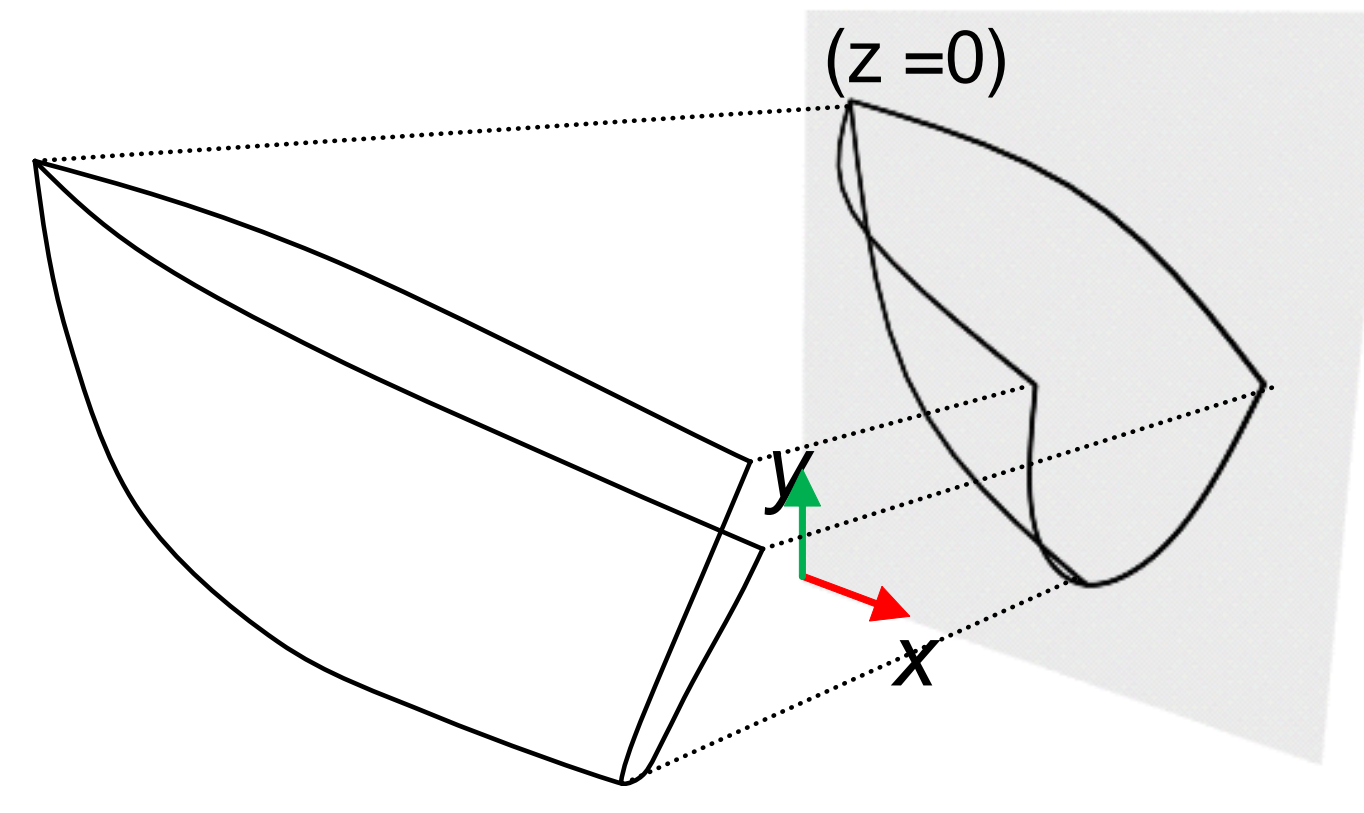




\section{Inferring mirror symmetric 3D curves from sketches}

- Assumptions:

- Mirror-symmetric shape composed of curves

- Orthogonal projection
(a)
$(z=0)$

$(\mathrm{z}=0)$
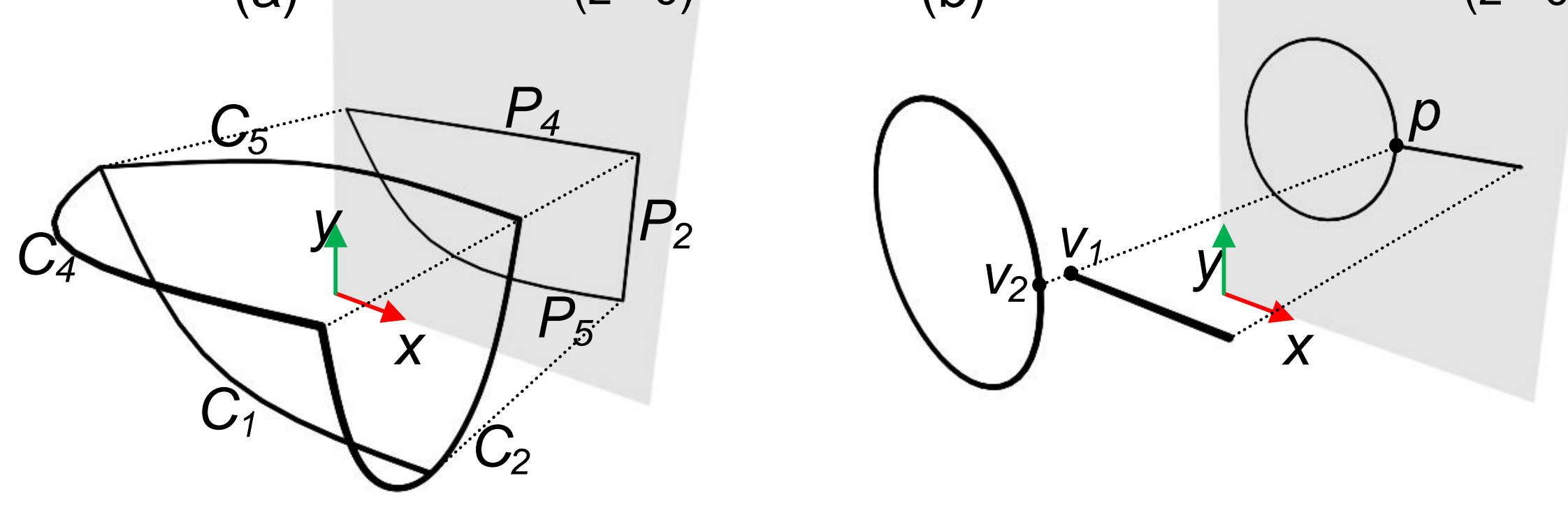


\section{Inferring mirror symmetric 3D curves from sketches}

\section{Overview}

- Finding pairs of symmetric

curves:

- 3D reconstruction:

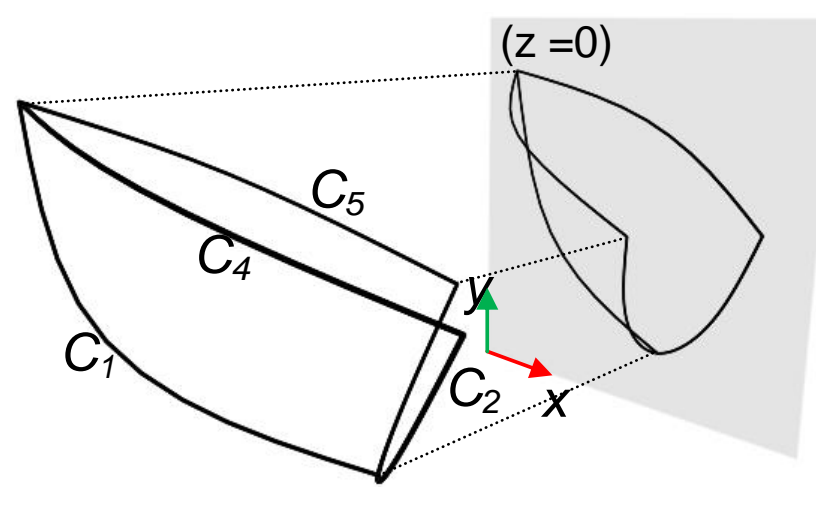




\section{Inferring mirror symmetric 3D curves from sketches}

Properties of symmetric polygons

- $\mathrm{P}$ and $\mathrm{P}^{\prime}$ are the orthogonal projections of a pair of symmetric 3D polygonal curves:

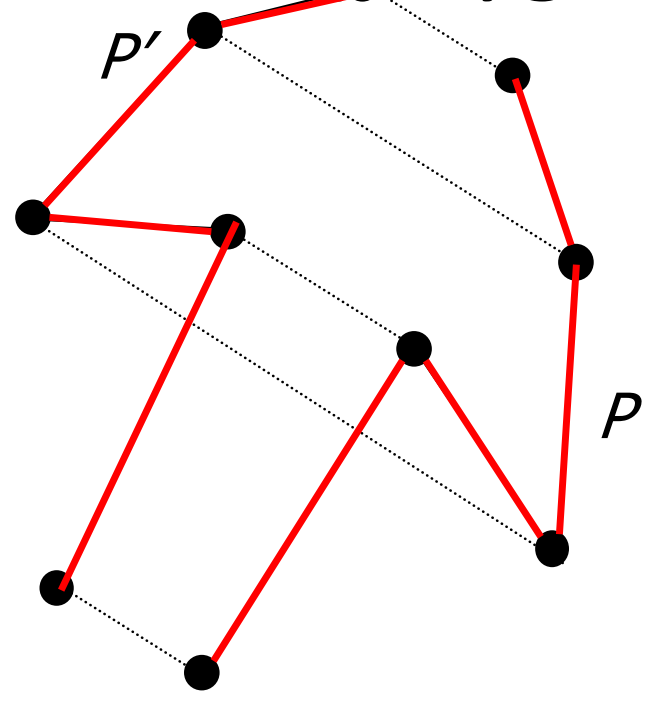




\section{Inferring mirror symmetric 3D curves from sketches}

How to find that P1 is symmetric to P2?
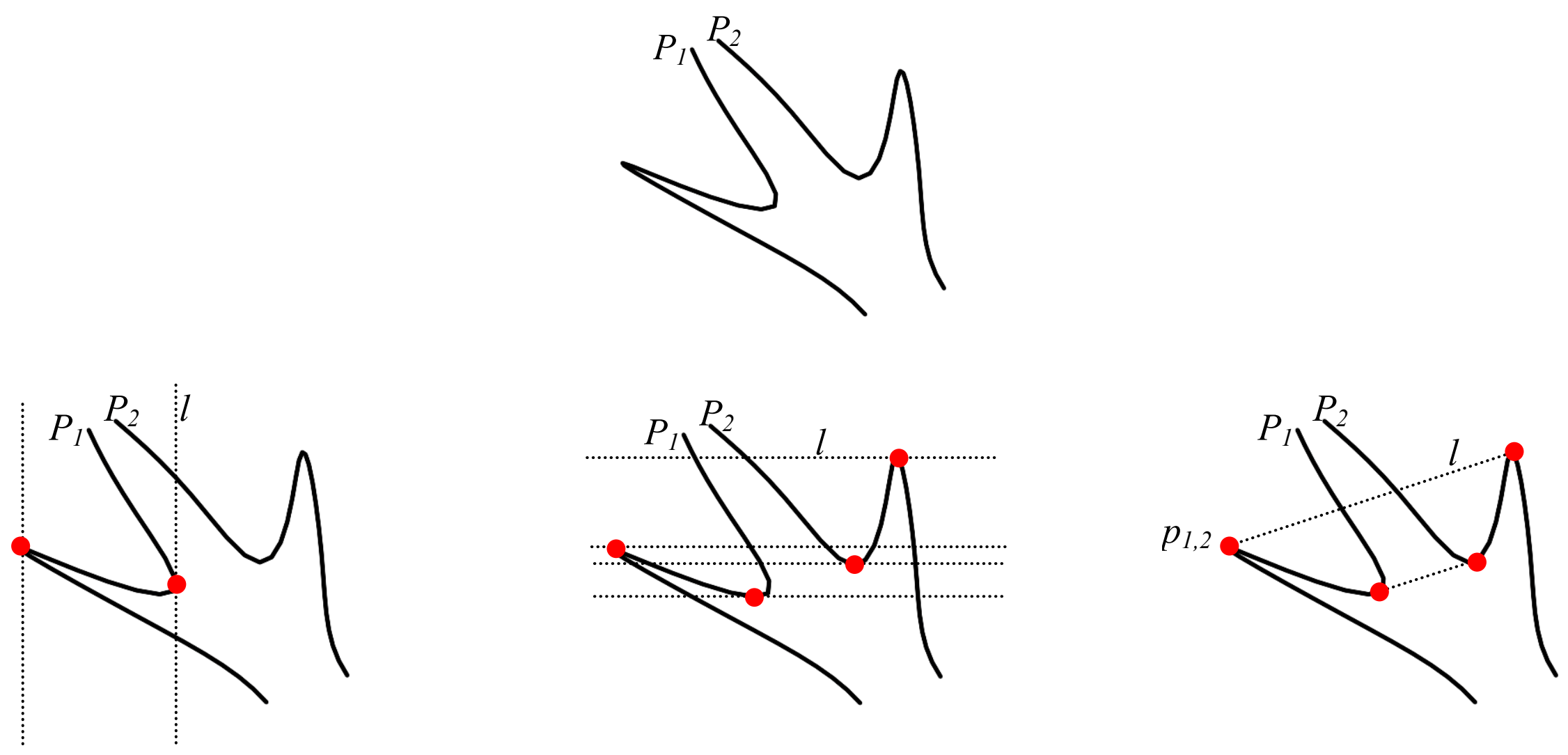

QP, SIGGRAPH 


\section{Inferring mirror symmetric 3D curves from sketches}

- A turn vertex is a vertex such that the two adjacent vertices are located in the same halfplane delimited by $\mathrm{l}$.

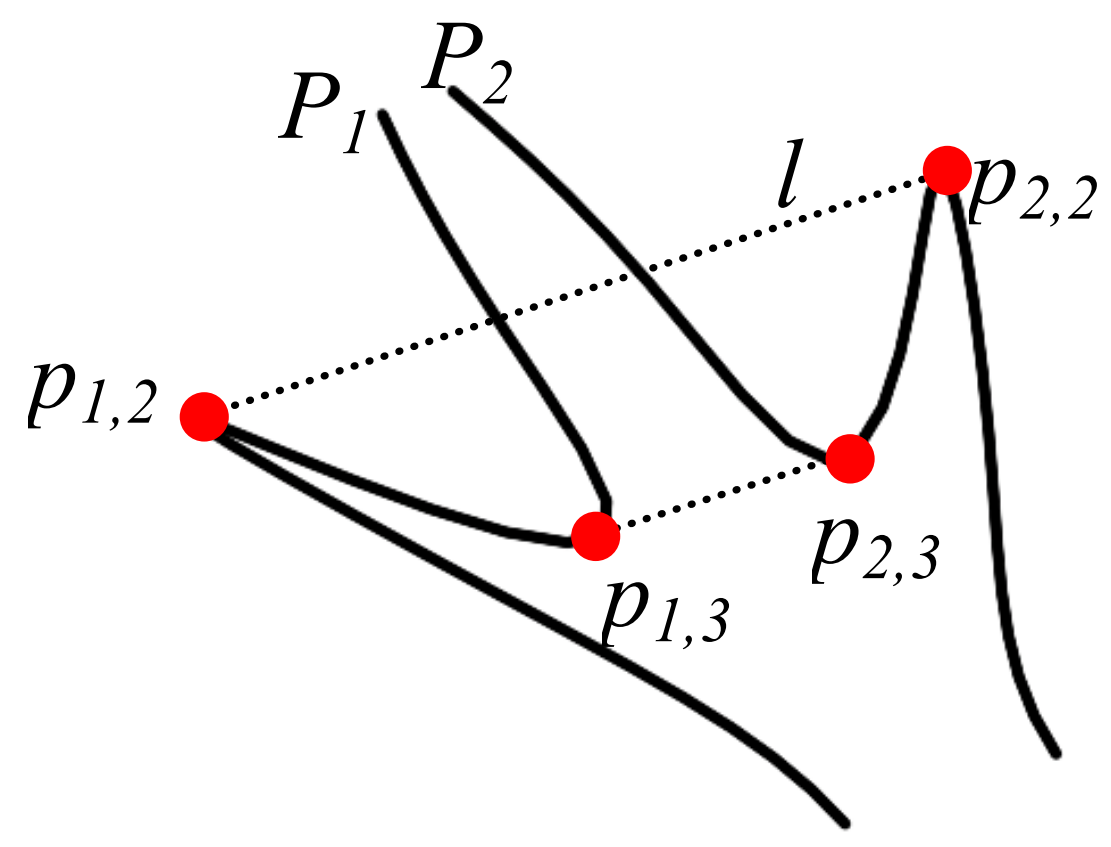




\section{Inferring mirror symmetric 3D curves from sketches}

- A turn vertex is a vertex such that the two adjacent vertices are located in the same halfplane delimited by $\mathrm{I}$.
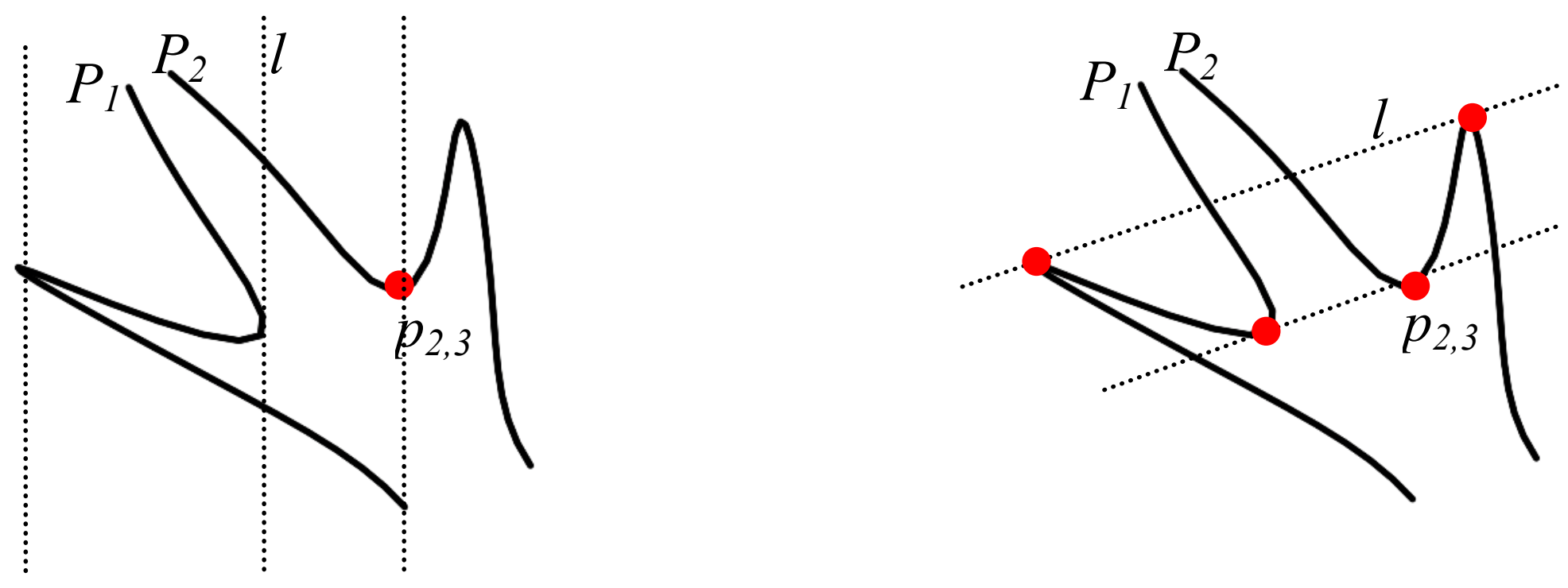


\section{Inferring mirror symmetric 3D curves from sketches}
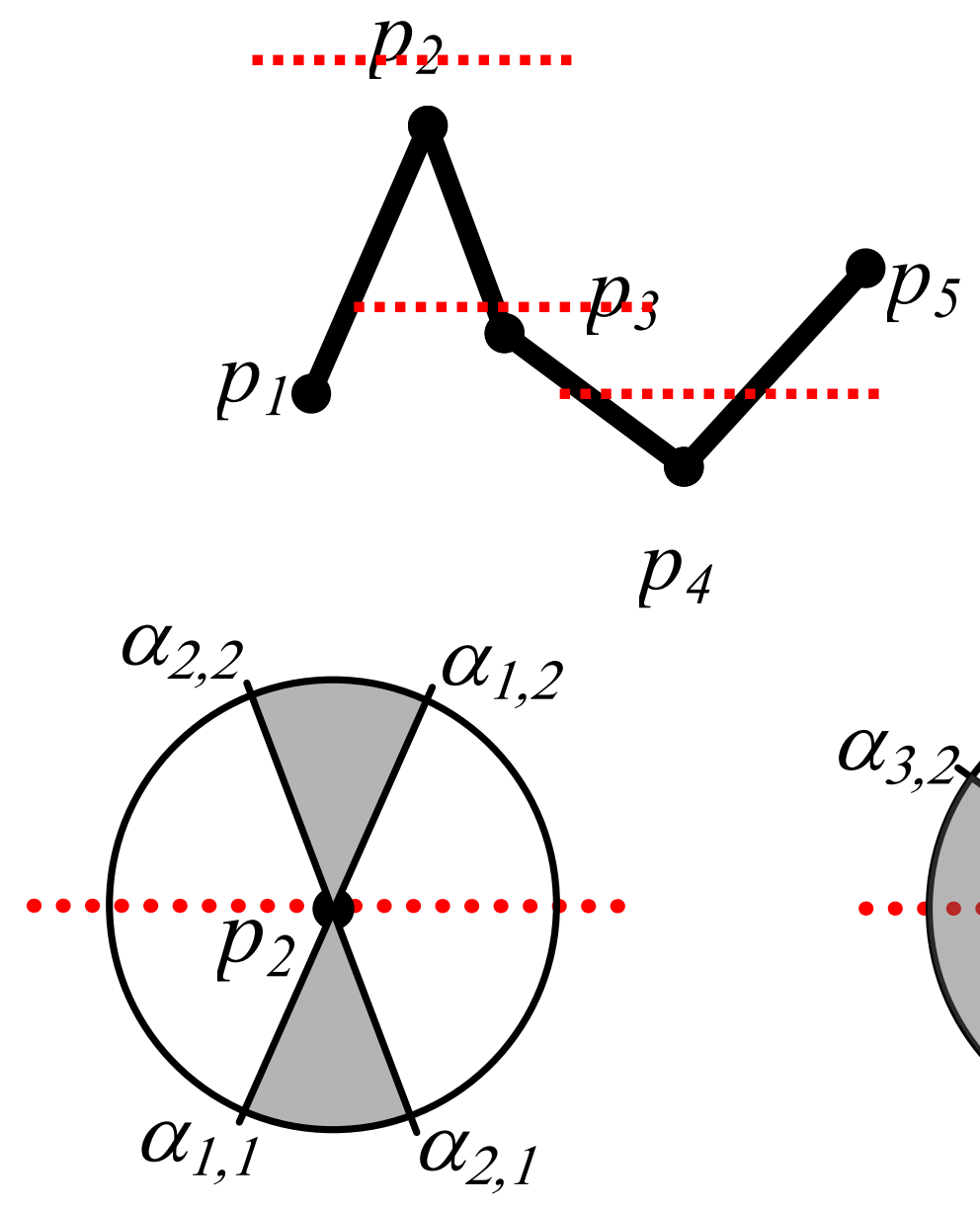

Qf SIGGRAPH
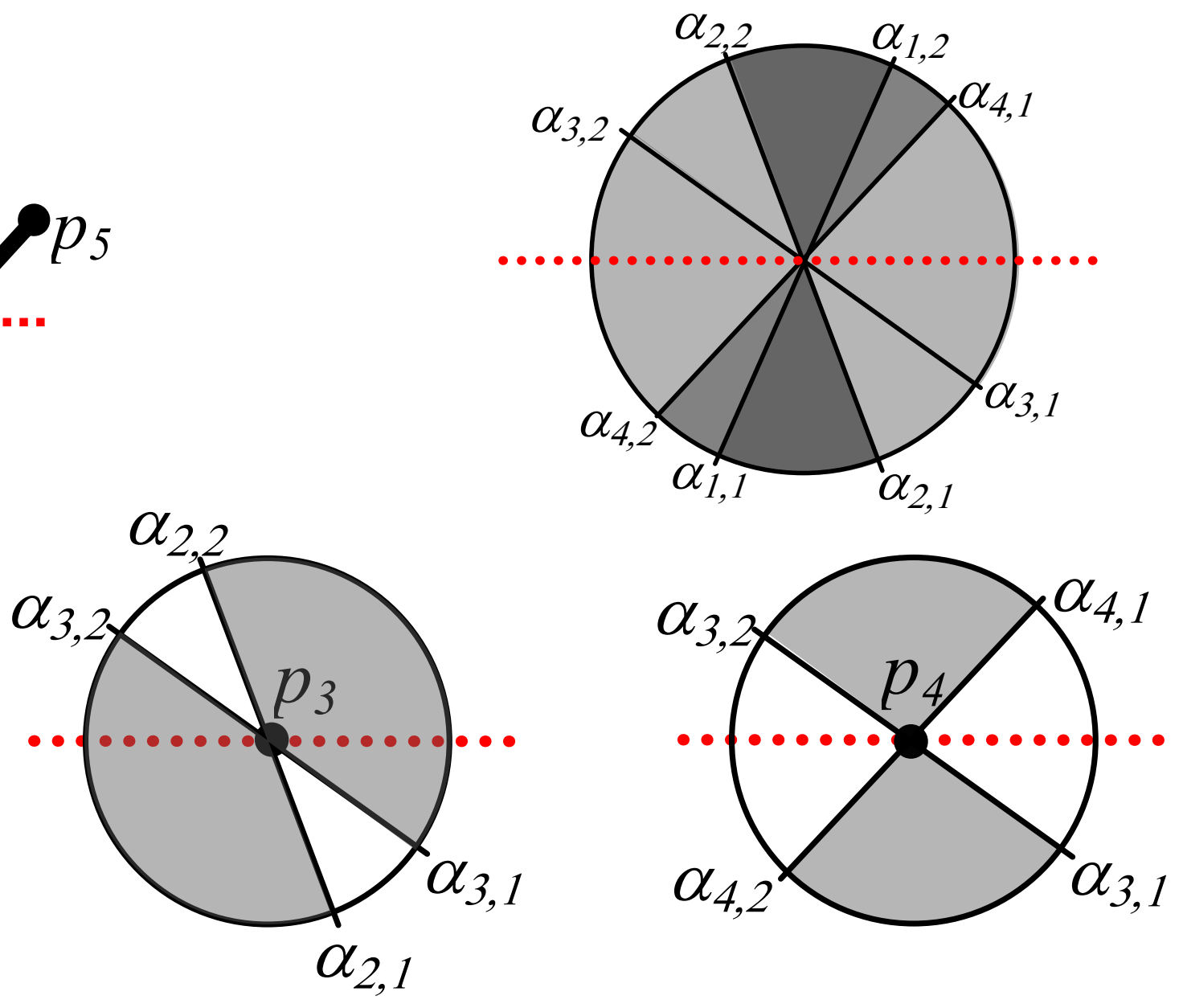


\section{Inferring mirror symmetric 3D curves from sketches}

Finding the symmetric curves

(a)

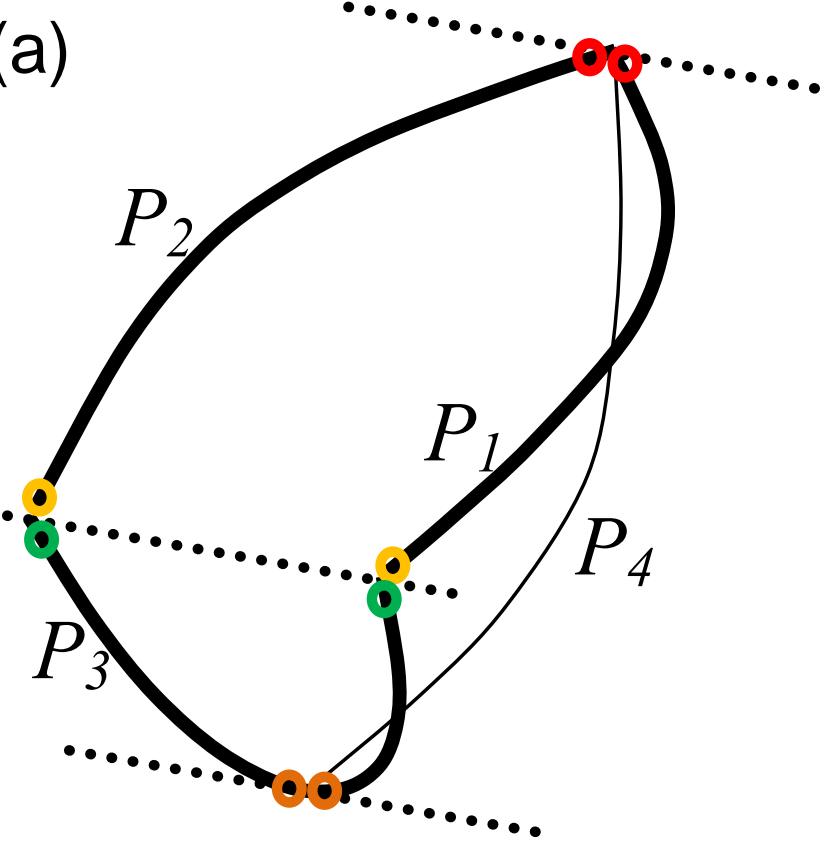

$P_{1}$ and $P_{2}$ symmetric

$P_{3}$ self-symmetric

$P_{4}$ non-symmetric

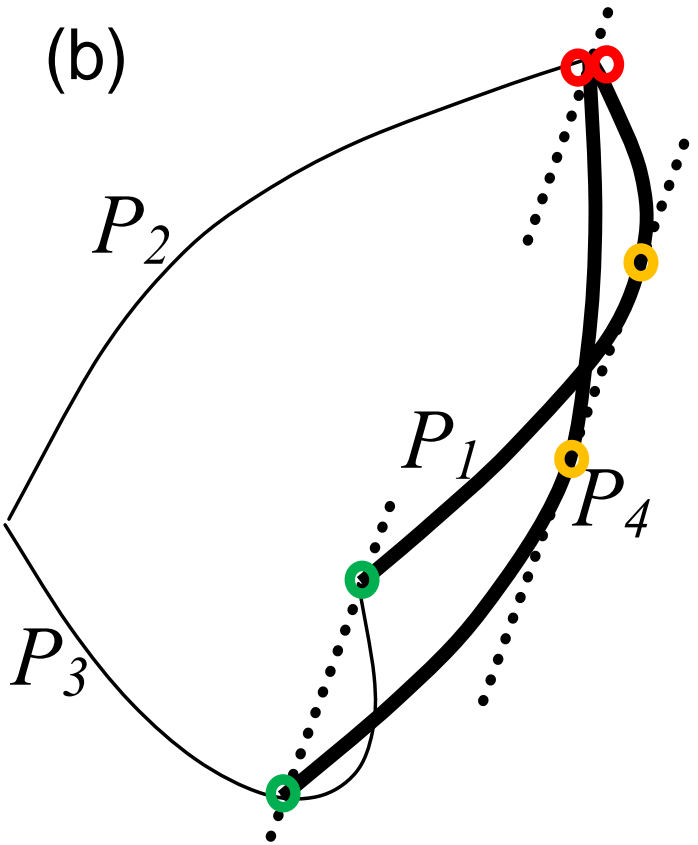

$P_{1}$ and $P_{4}$ symmetric

$P_{3}$ and $P_{2}$ non-

symmetric

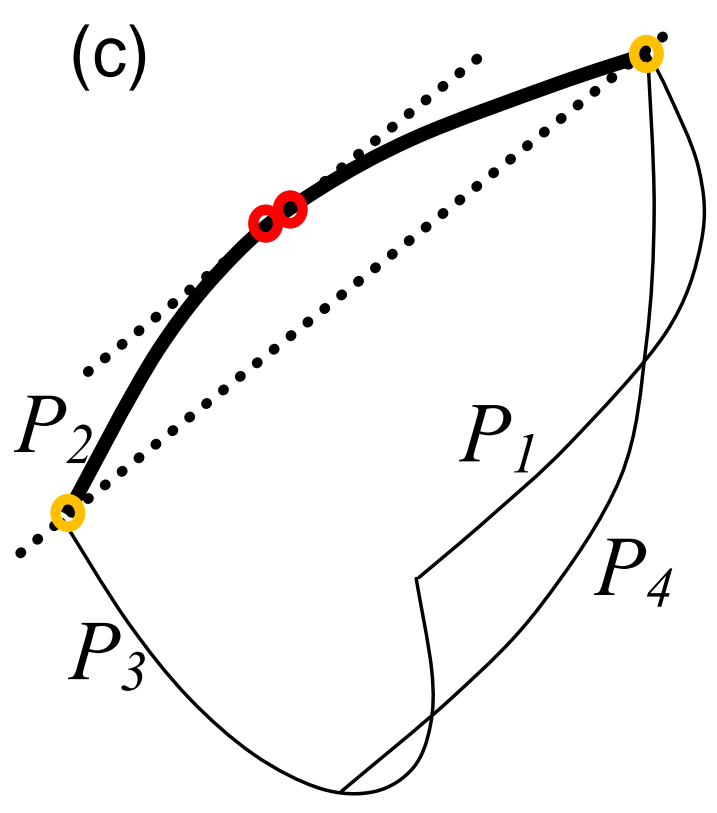

$P_{2}$ self-symmetric $P_{1}, P_{3}$ and $P_{4}$ nonsymmetric 


\section{Inferring mirror symmetric 3D curves from sketches}

Computing the symmetry relationship

- Unnatural 3D reconstruction
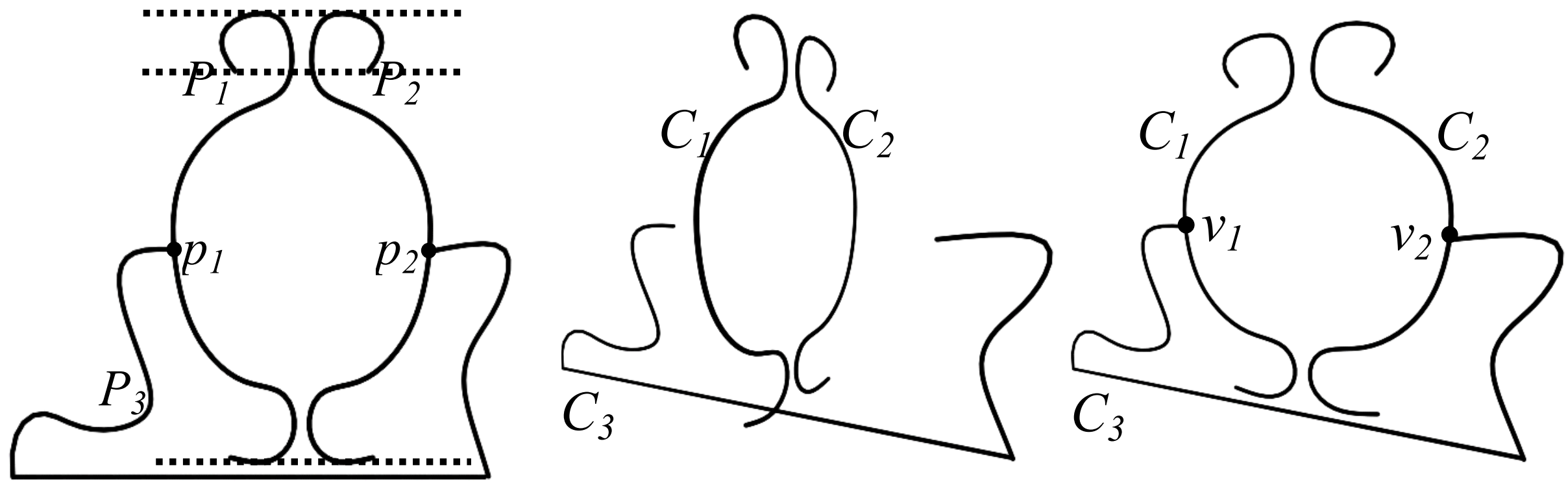


\section{Inferring mirror symmetric 3D curves from sketches}

- Exploiting the curve connectivity and the generic viewpoint assumption
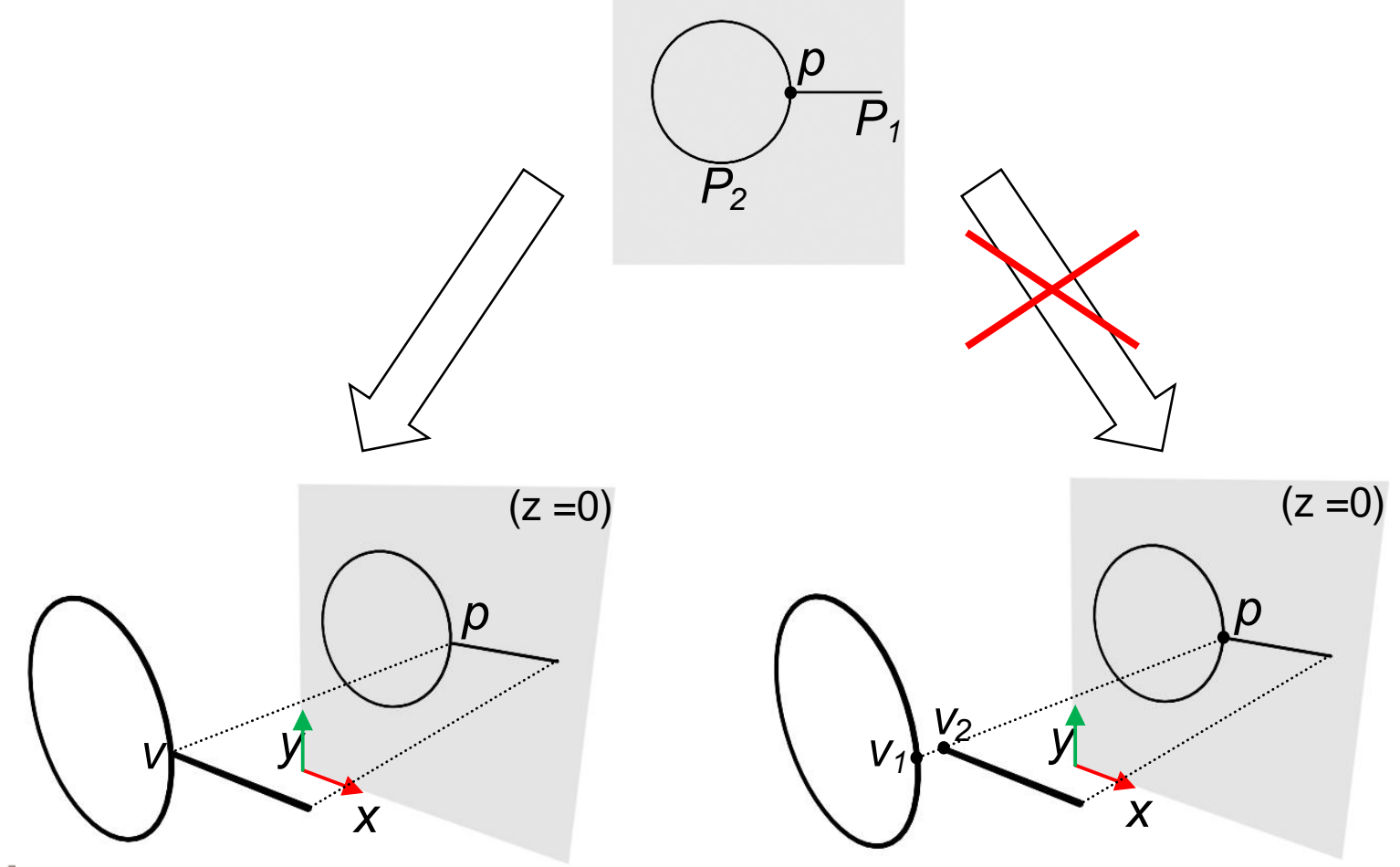


\section{Inferring mirror symmetric 3D curves from sketches}

- The curve connectivity is not sufficient to uniquely define the symmetry relationship.
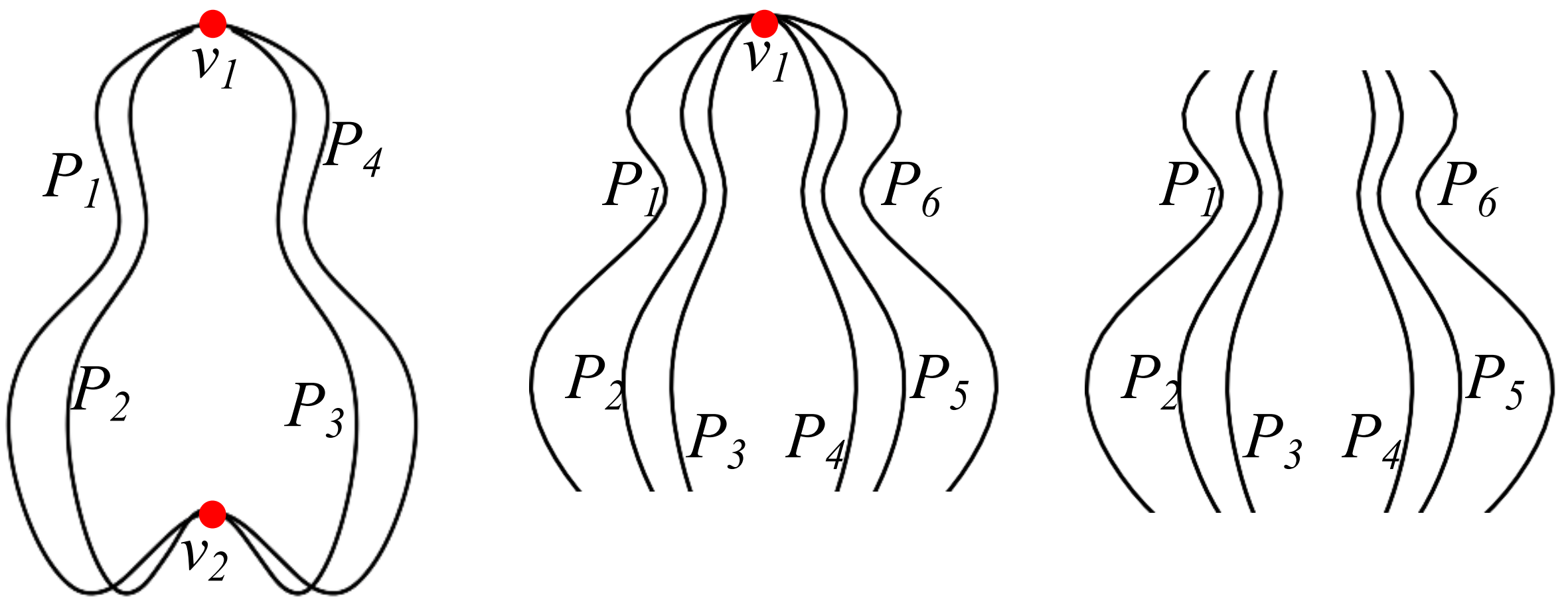

Q?, SIGGRAPH 


\section{Inferring mirror symmetric 3D curves from sketches}

Maximizing the compactness of the reconstructed curves:

$$
C(O)=\frac{V(O)^{2}}{S(O)^{3}}
$$
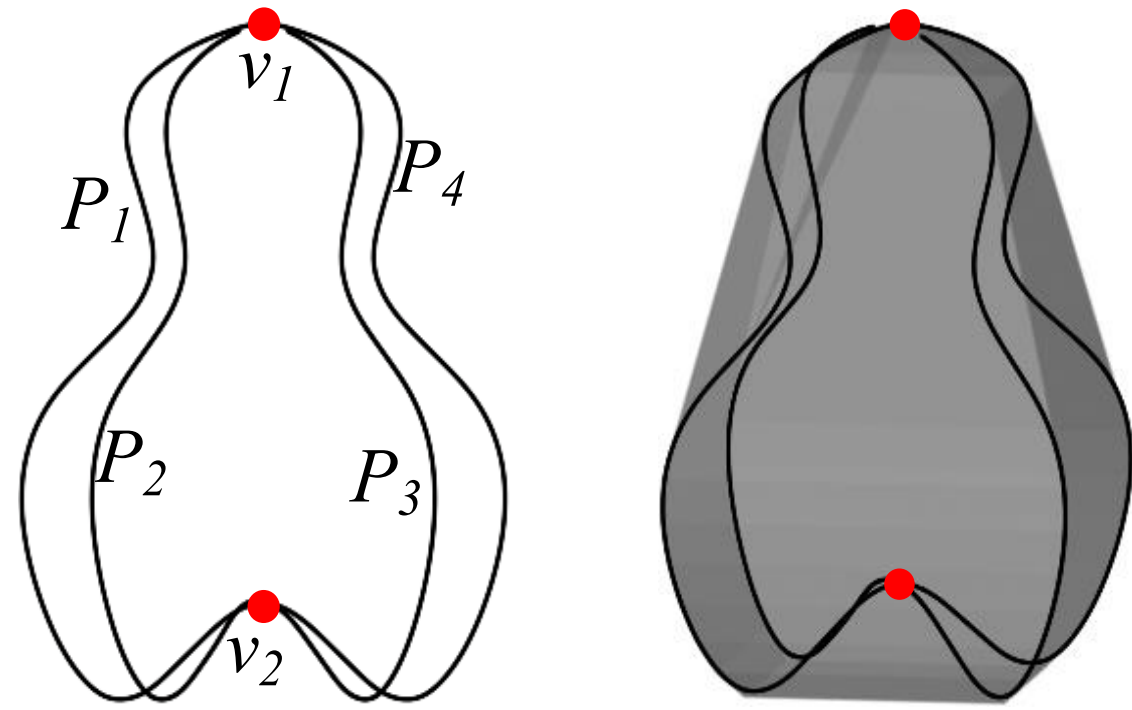

Li Y, Pizlo Z, Steinman RM. A computational model that recovers the 3D shape 33 of an object from a single 2D retinal representation. Vision Research. 2009; 34 49(9):979-91. 


\section{Inferring mirror symmetric 3D curves from sketches}
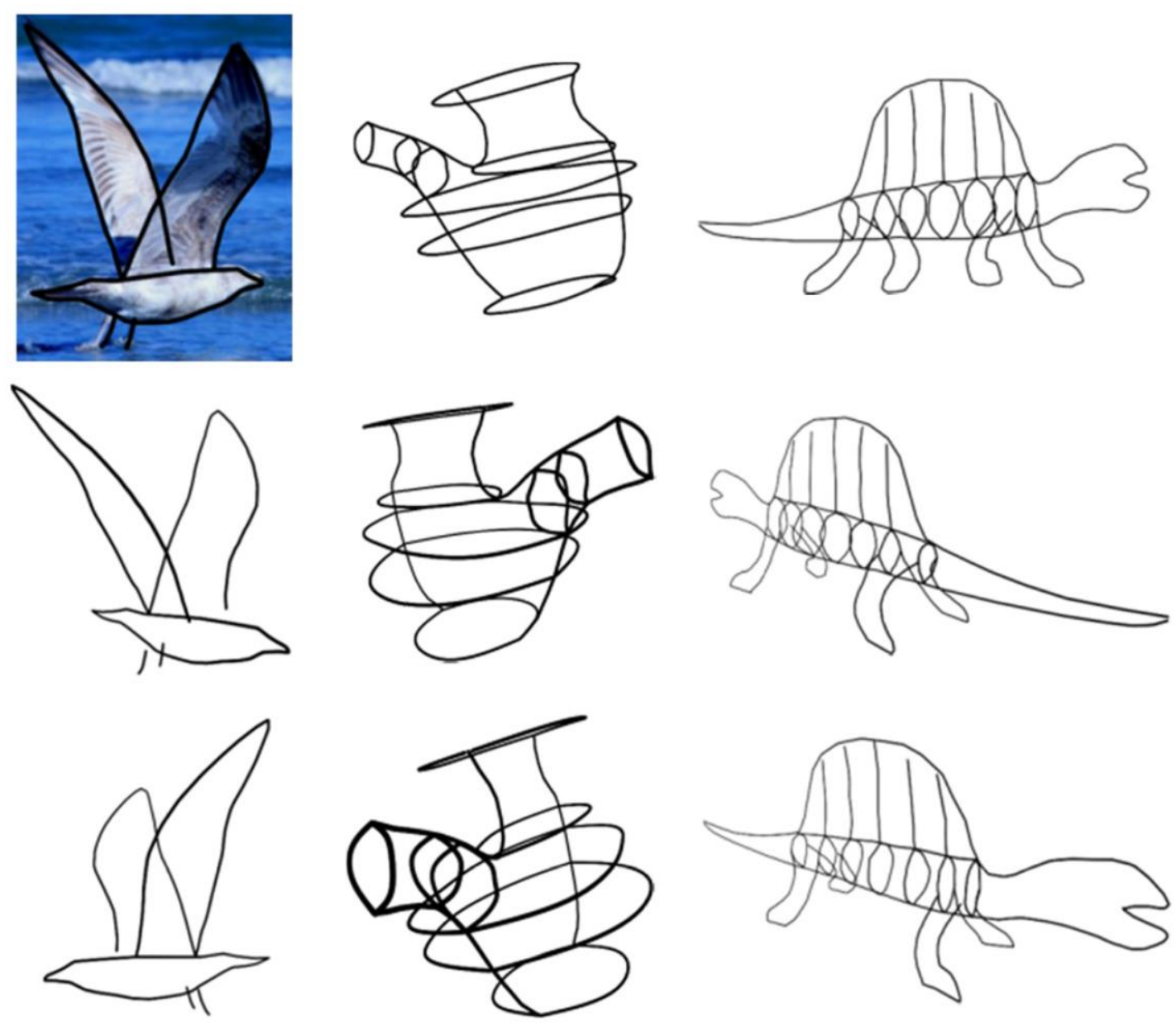

Q9. SIGGRAPH 


\section{Open problems in sketch-based modeling}

- 3D reconstruction from sketches

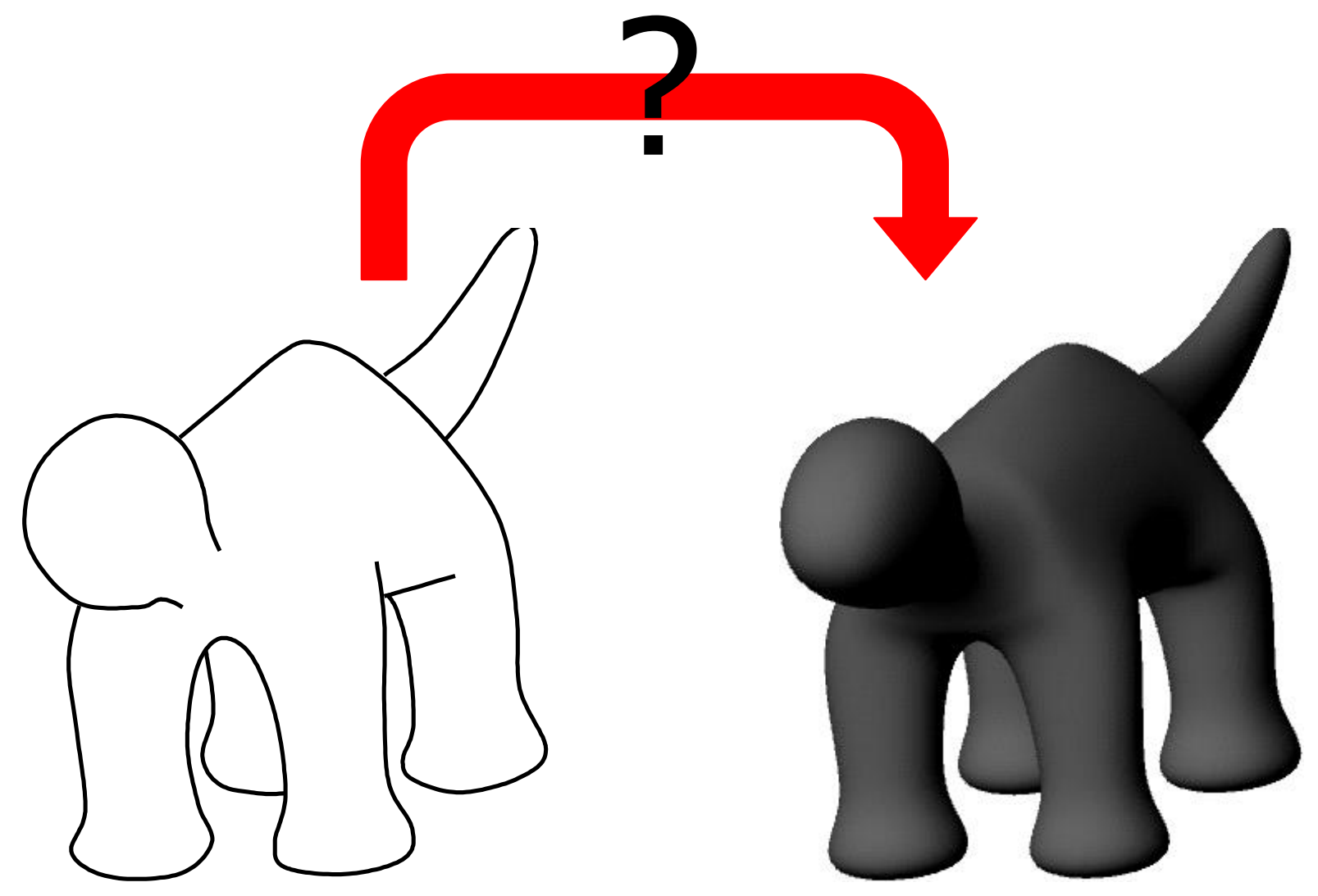




\section{Open problems in sketch-based modeling}

Silhouette completion

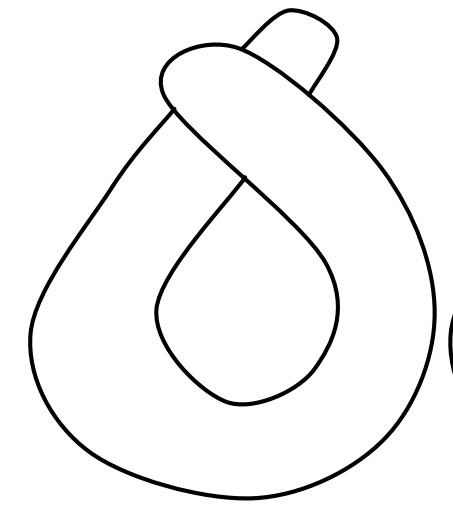

(a)

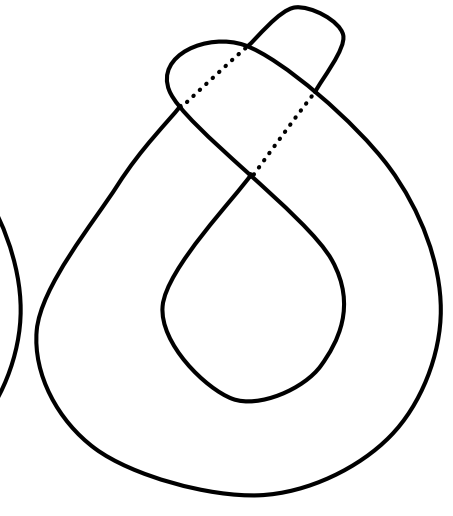

(b)

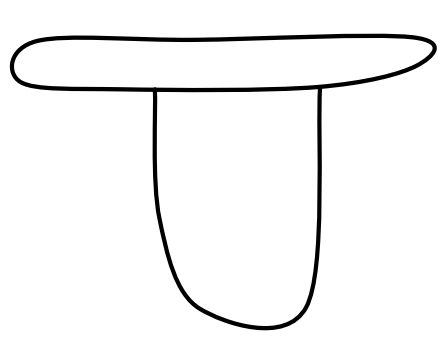

(c)

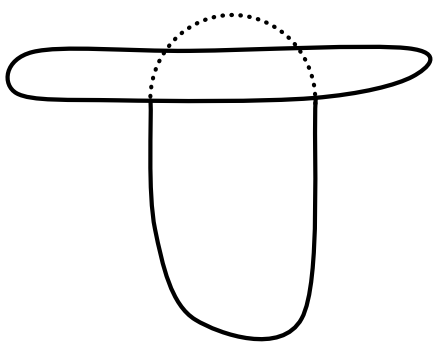

(d)

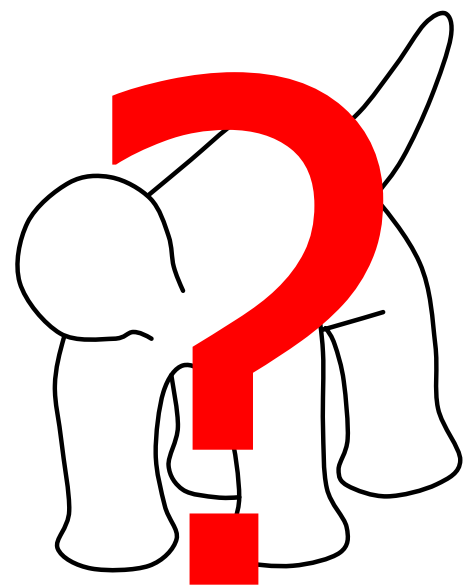

(e)

Lance R. Williams: Topological Reconstruction of a Smooth Manifold-Solid from Its Occluding Contour. International Journal of Computer Vision 23(1): 93-108 (1997) 


\section{Open problems in sketch-based modeling}

3D Reconstruction

- Input: the 2D sketch composed of polygonal curves in a plane

- Output: a shape such that its orthogonal projection matches the input sketch An infinite number of solutions !

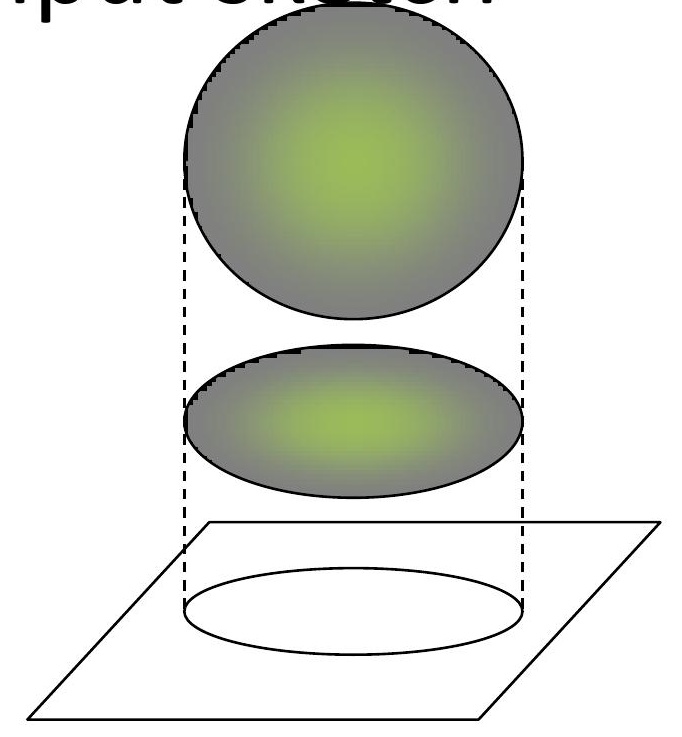




\section{Open problems in sketch-based modeling}

- How to select the best solution ?

- According to Li et al., four criteria:

- Symmetry

- Planarity

- Maximum compactness

- Minimum surface

Li, Y., Pizlo, Z. \& Steinman, R.M. "A computational model that recovers the 3D shape of an object from a single 2D retinal representation", Vision Research, Volume 49, Issue 9, 11 May 2009, Pages 979-991 


\section{Open problems in sketch-based modeling}

- Symmetry refers to the mirror-symmetry of the object.

Most of people will interpret this as a sphere

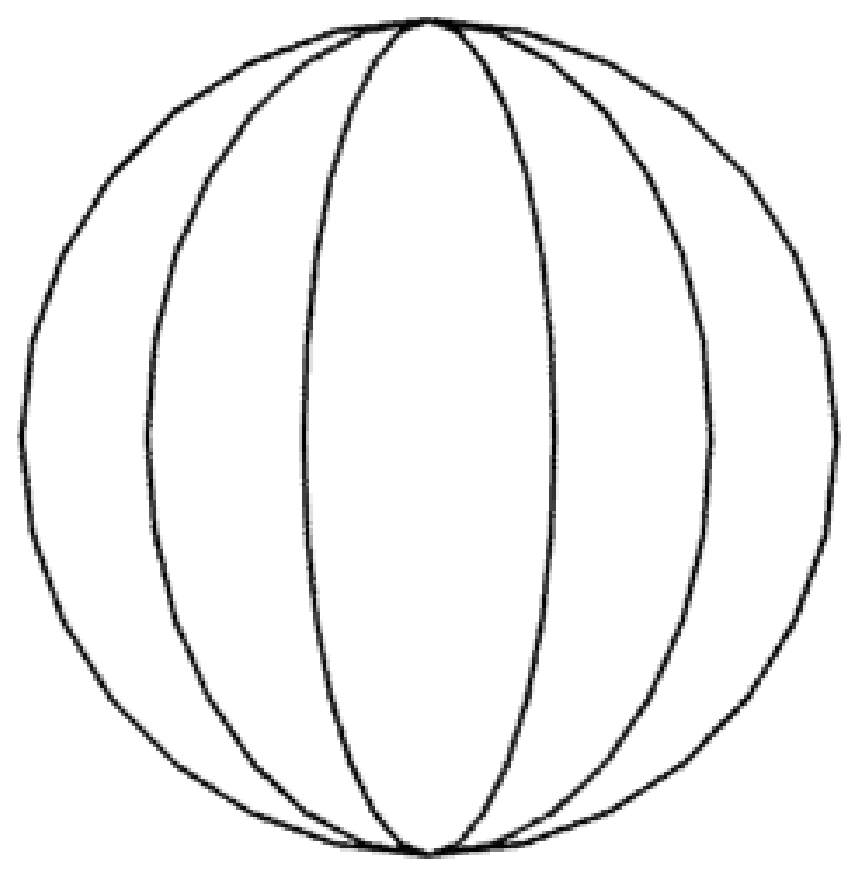




\section{Open problems in sketch-based modeling}

- Planarity refers to the planarity of the contours of the object.
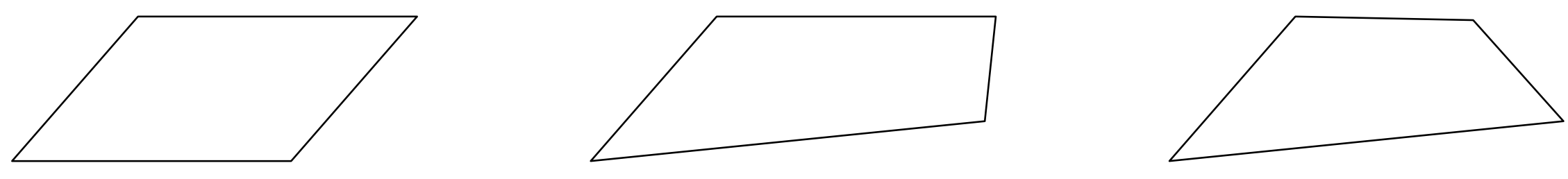


\section{Open problems in sketch-based modeling}

- Compactness is defined as $\mathrm{V}^{2} / \mathrm{S}^{3}$ where $\mathrm{V}$ is the object's volume and $S$ is the object's surface area.

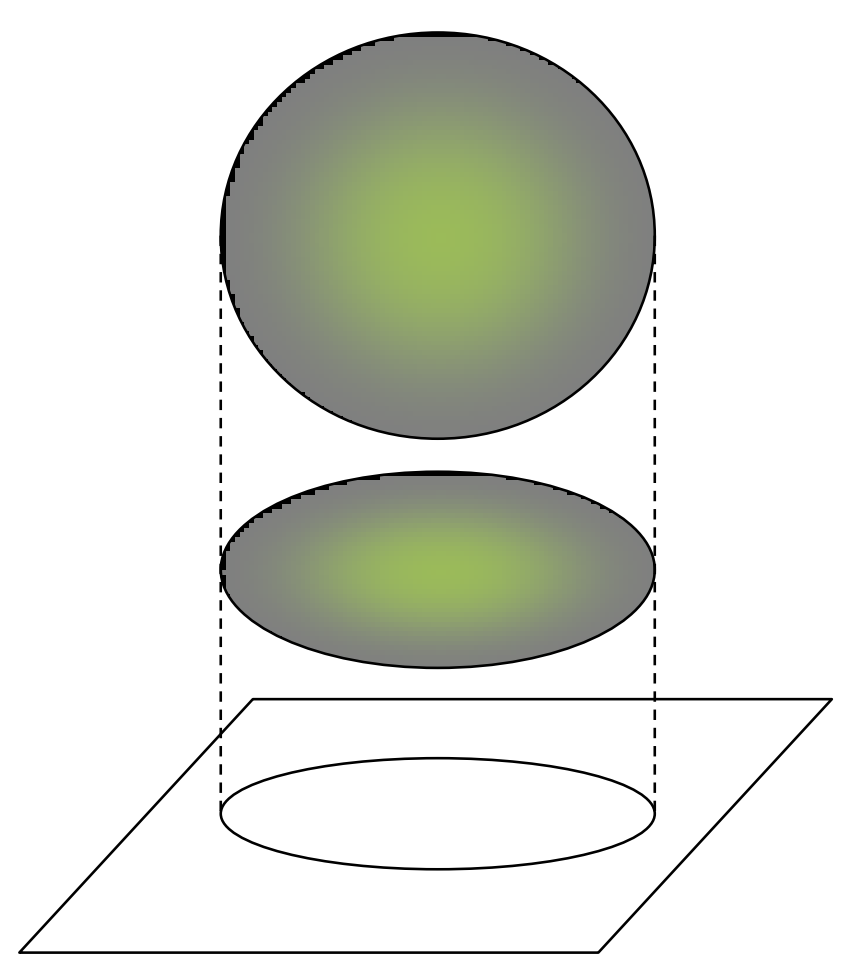




\section{D Reconstruction}

- Minimum surface is defined as the minimum of the total surface area.

-> Recover a 3D shape, which has the smallest range in depth 


\section{Sketch-based editing}

\section{Yotam Gingold}




\section{Editing operations}

- Cutting (we saw earlier)

- Deform by sketching new silhouettes

- Edit relief by sketching shading

- Digital sculpting (ZBrush, etc) - won't talk about 


\section{A Sketch-Based Interface for Detail-Preserving Mesh Editing [Nealen et al. 2005]}

\section{- Silhouette editing}

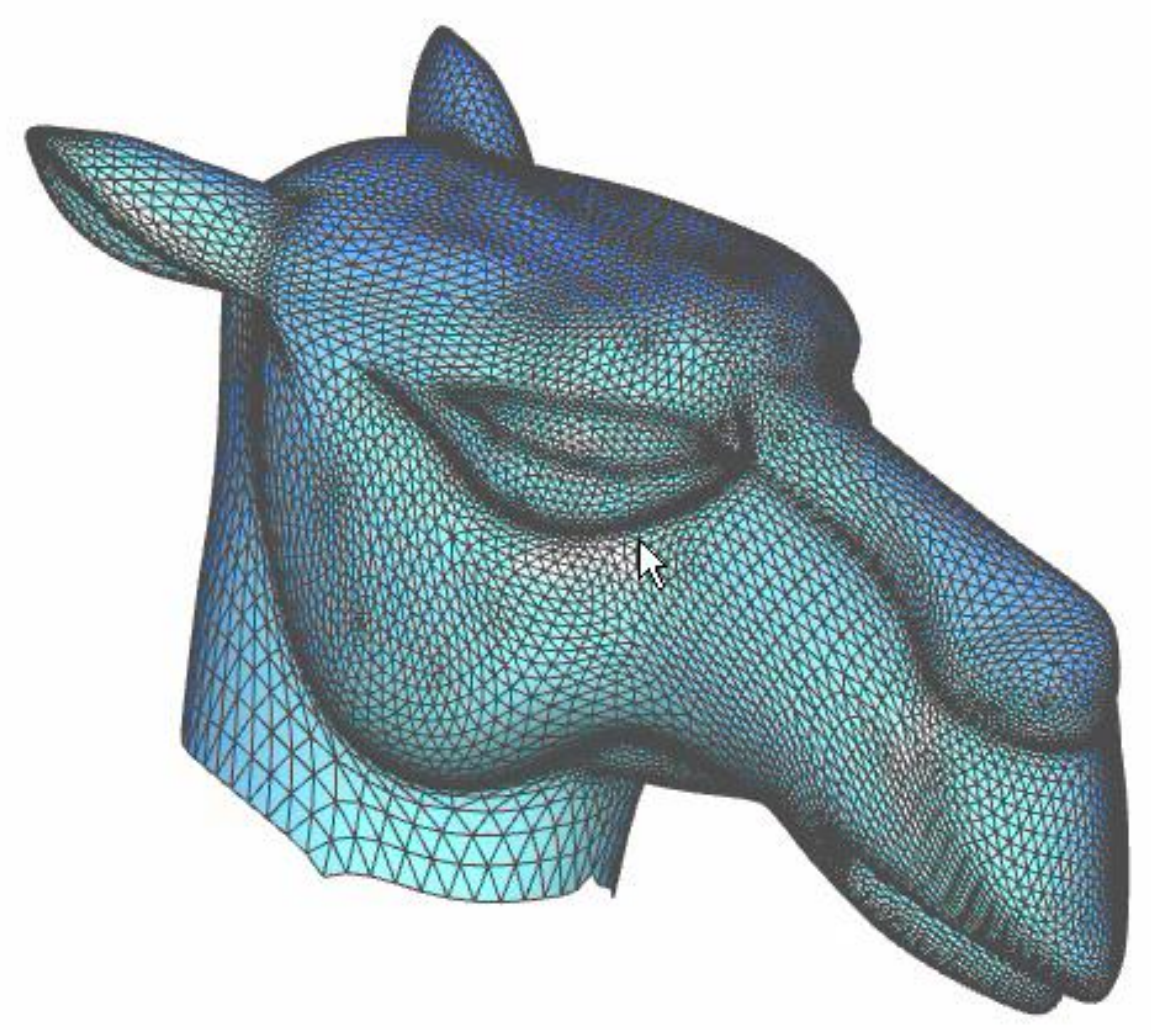




\section{A Sketch-Based Interface for Detail-Preserving Mesh Editing [Nealen et al. 2005]}

- Silhouette creation 


\section{A Sketch-Based Interface for Detail-Preserving Mesh Editing [Nealen et al. 2005]}

- To edit a silhouette:

- Parameterize silhouette edges 


\section{A Sketch-Based Interface for Detail-Preserving Mesh Editing [Nealen et al. 2005]}

- To edit a silhouette:

- Parameterize silhouette edges

- Parameterize sketch 


\section{A Sketch-Based Interface for Detail-Preserving Mesh Editing [Nealen et al. 2005]}

- To edit a silhouette:

- Parameterize silhouette edges

- Parameterize sketch

- Find correspondences

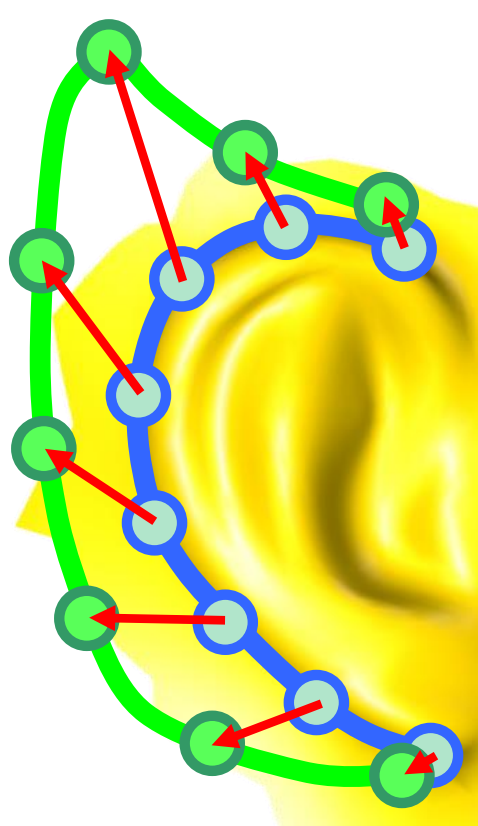




\section{A Sketch-Based Interface for Detail-Preserving Mesh Editing [Nealen et al. 2005]}

- To edit a silhouette:

- Parameterize silhouette edges

- Parameterize sketch

- Find correspondences

- Use as xy position constraints (keep z unchanged)

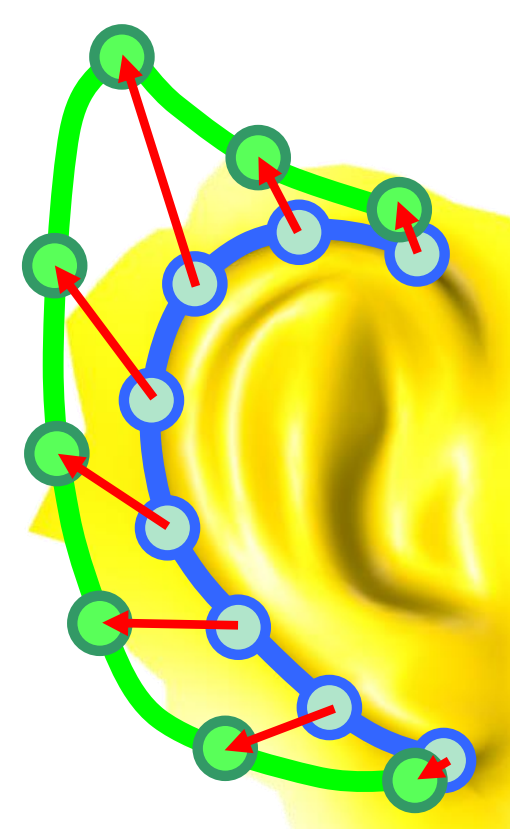




\section{A Sketch-Based Interface for Detail-Preserving Mesh Editing [Nealen et al. 2005]}

- To edit a silhouette:

- Parameterize silhouette edges

- Parameterize sketch

- Find correspondences

- Use as xy position constraints (keep $z$ unchanged)

- Minimize Laplacian Surface Editing energy [Sorkine et al. 2004]

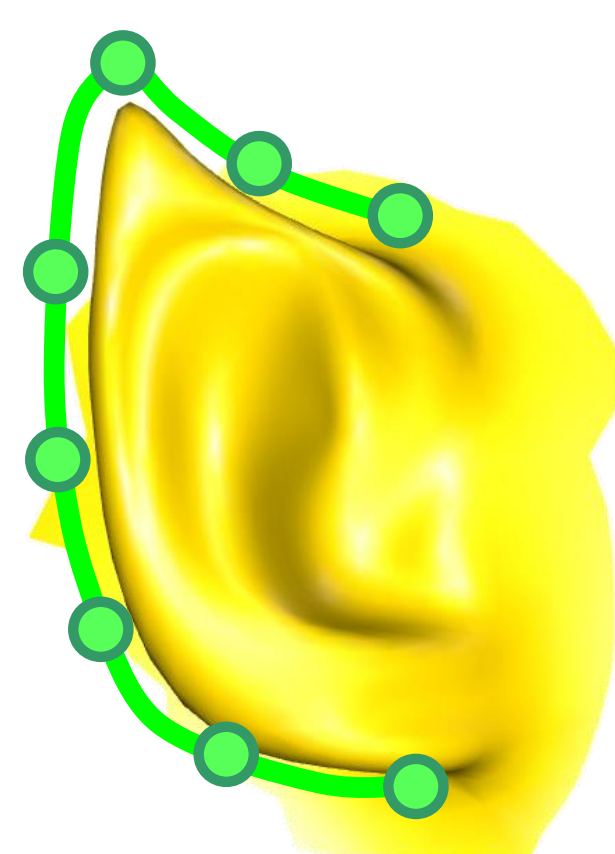




\section{Surface relief editing by sketching shading}

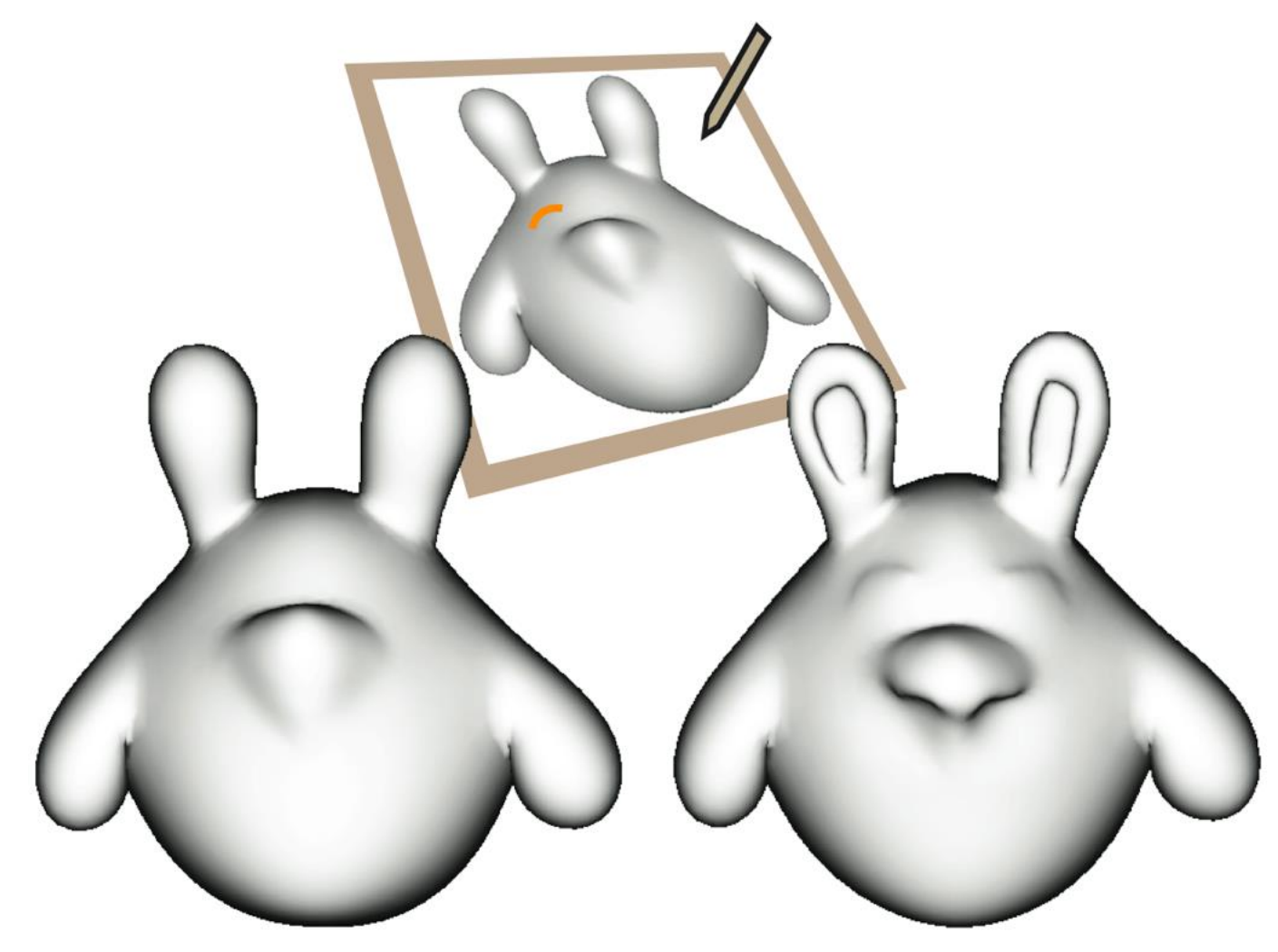

Shading-Based Surface Editing [Gingold and Zorin 2008] 


\section{Shading}

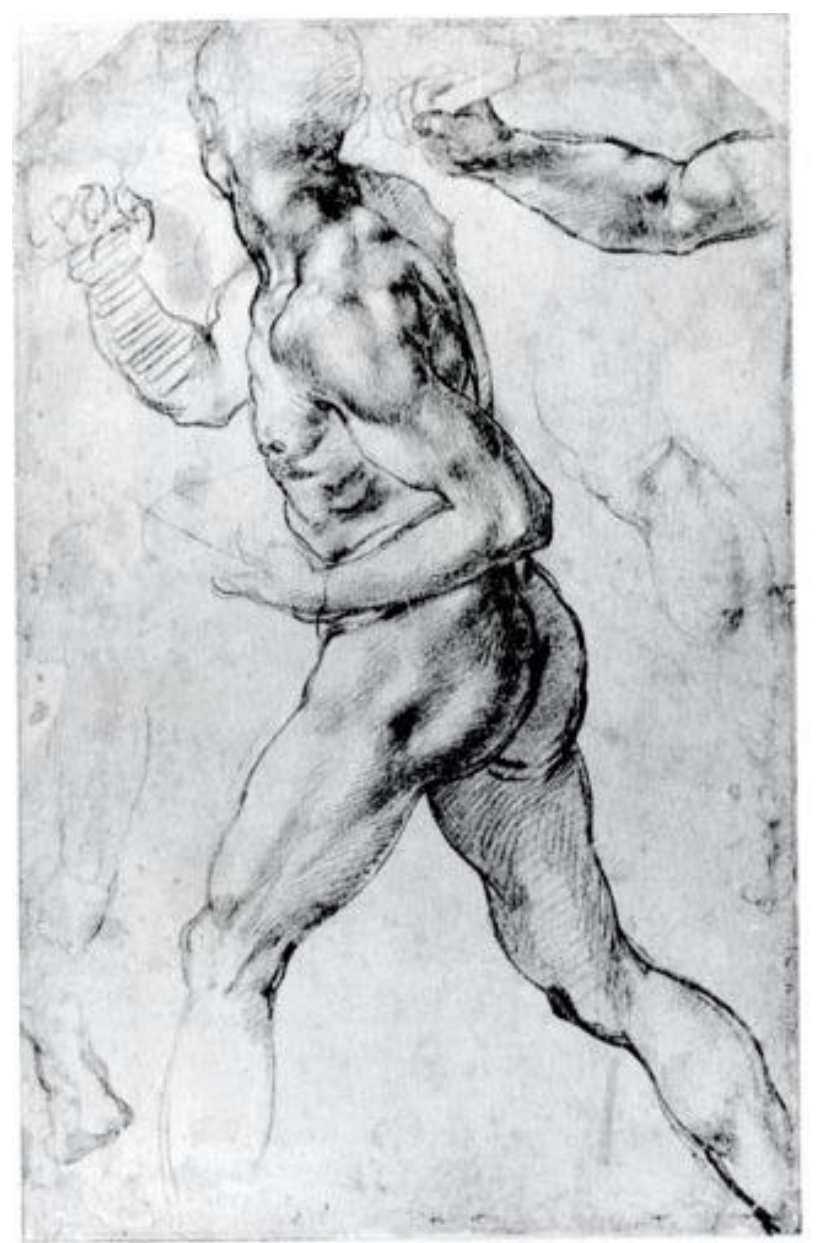

[Michelangelo]

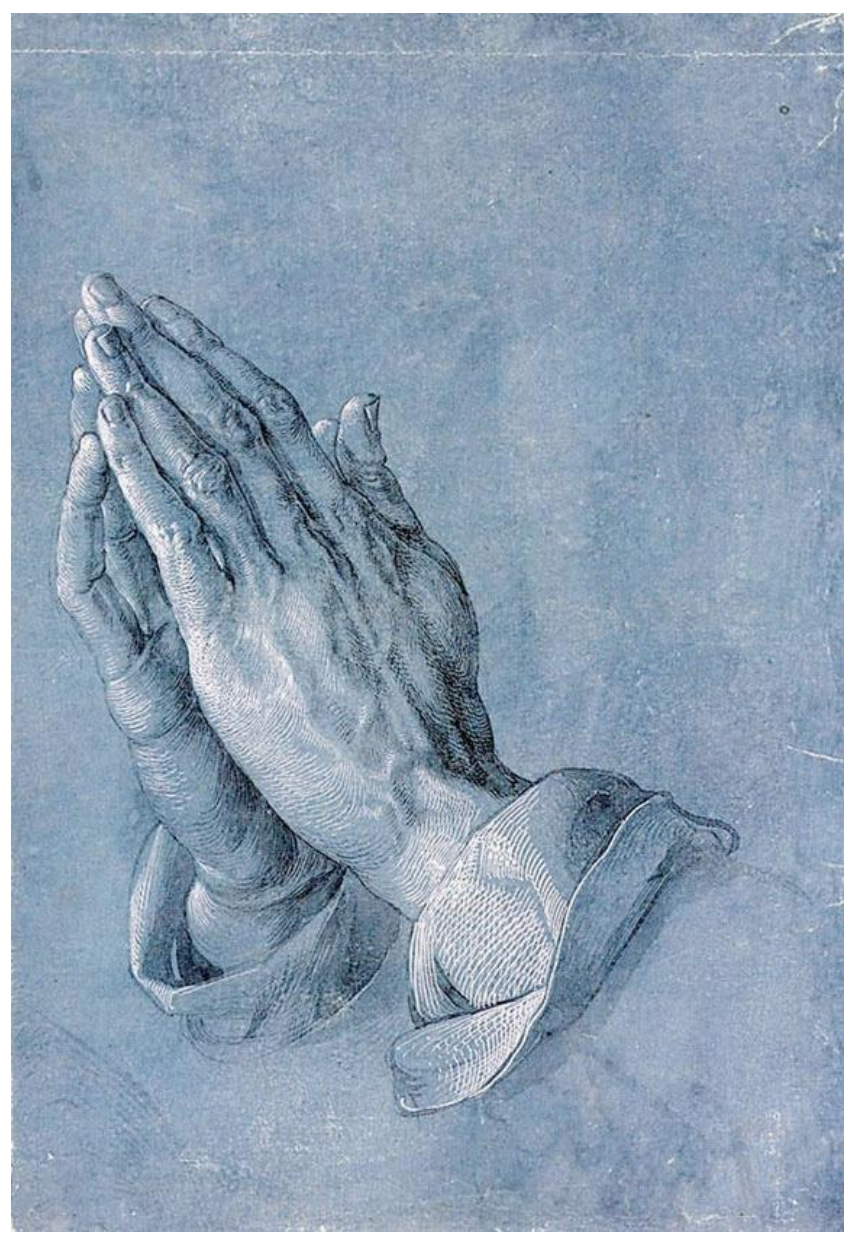

[Dürer]

Q Q SIGGRAPH 


\section{Shaded 3D Models}

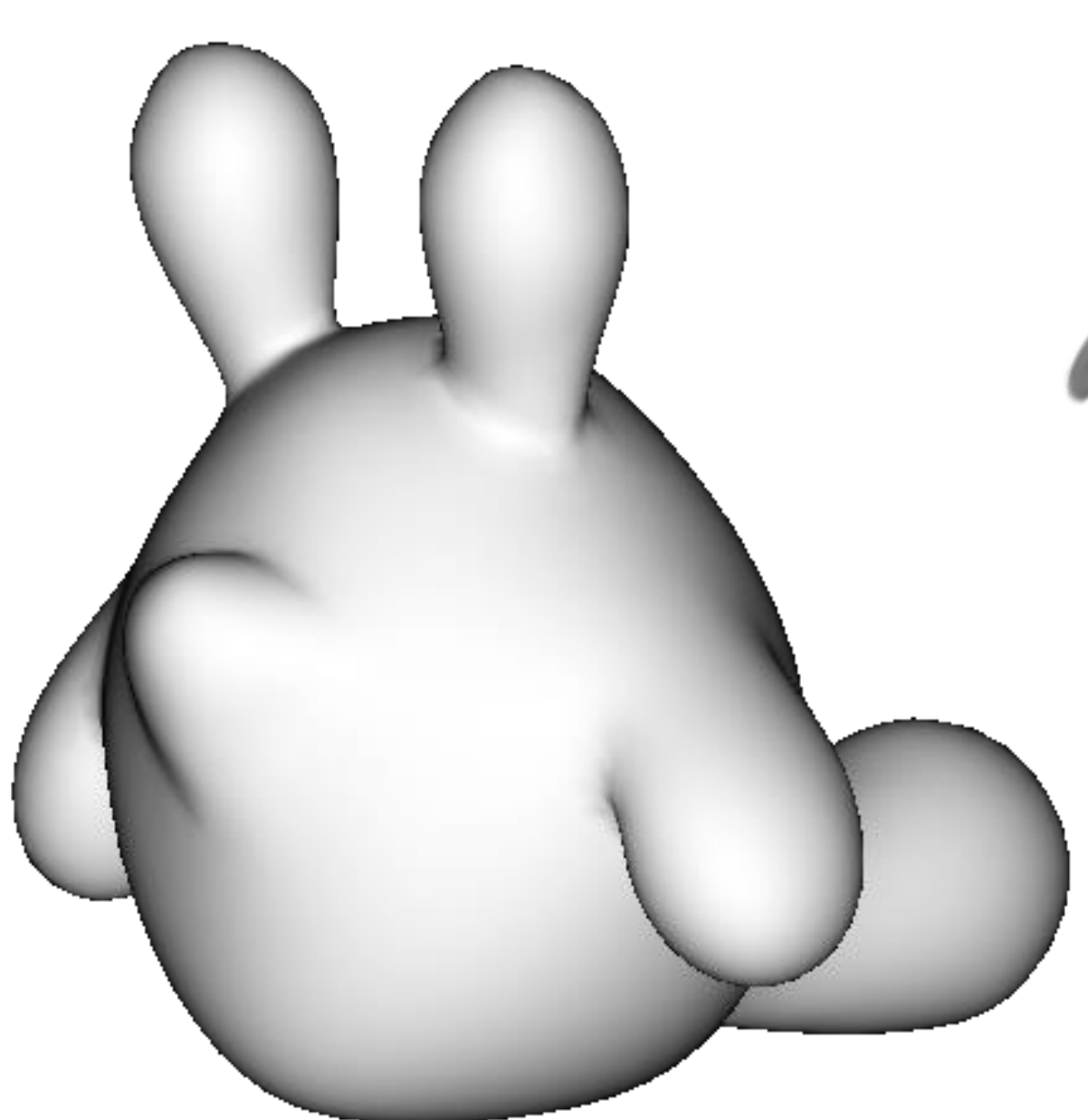

FiberMesh [Nealen et al. 2007]

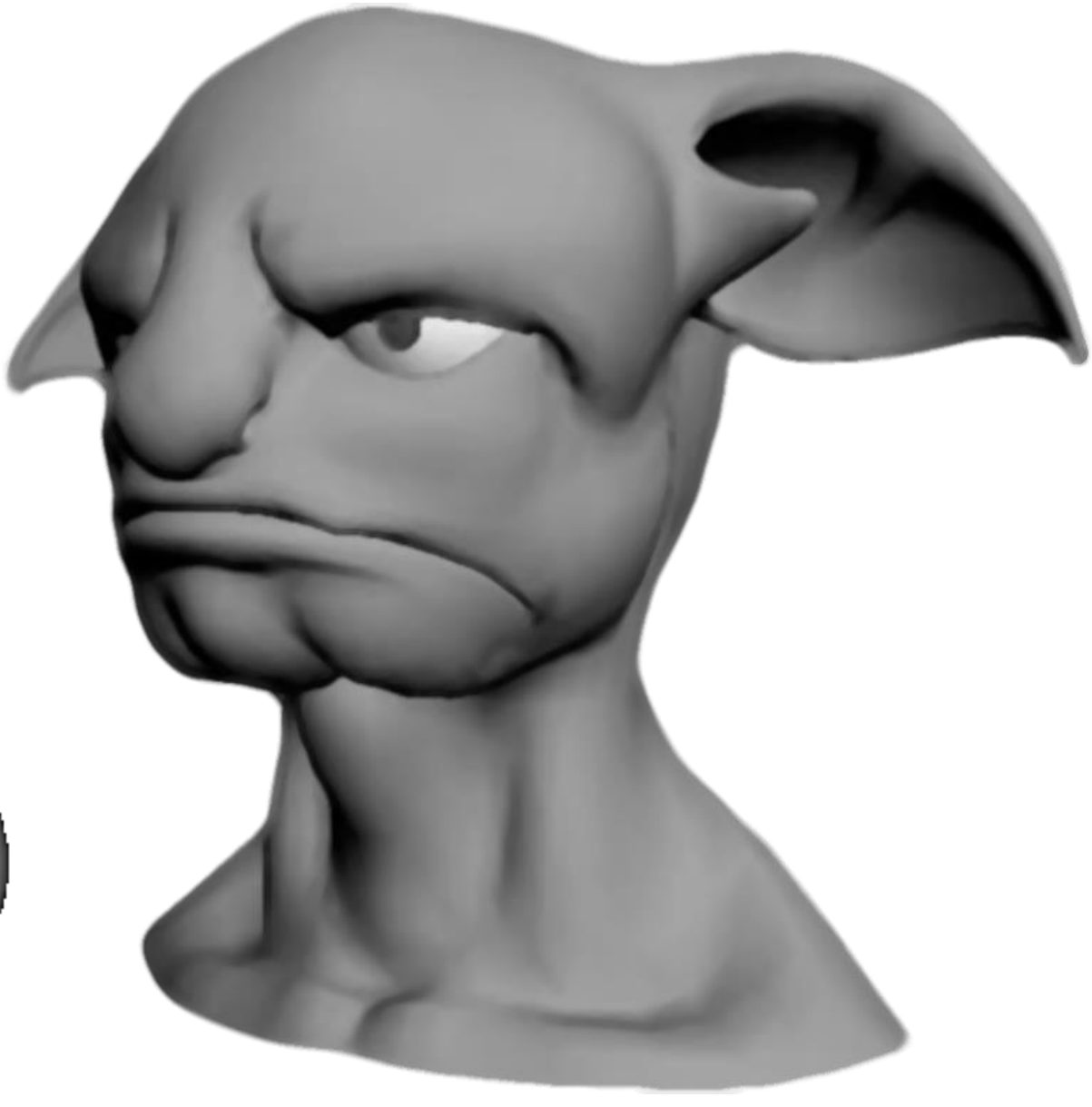

(C) Miguel Ângelo

OQ. SIGGRAPH

ASIA 2016 


\section{Approach}

Obtain a new 3D model by shading over an existing one.

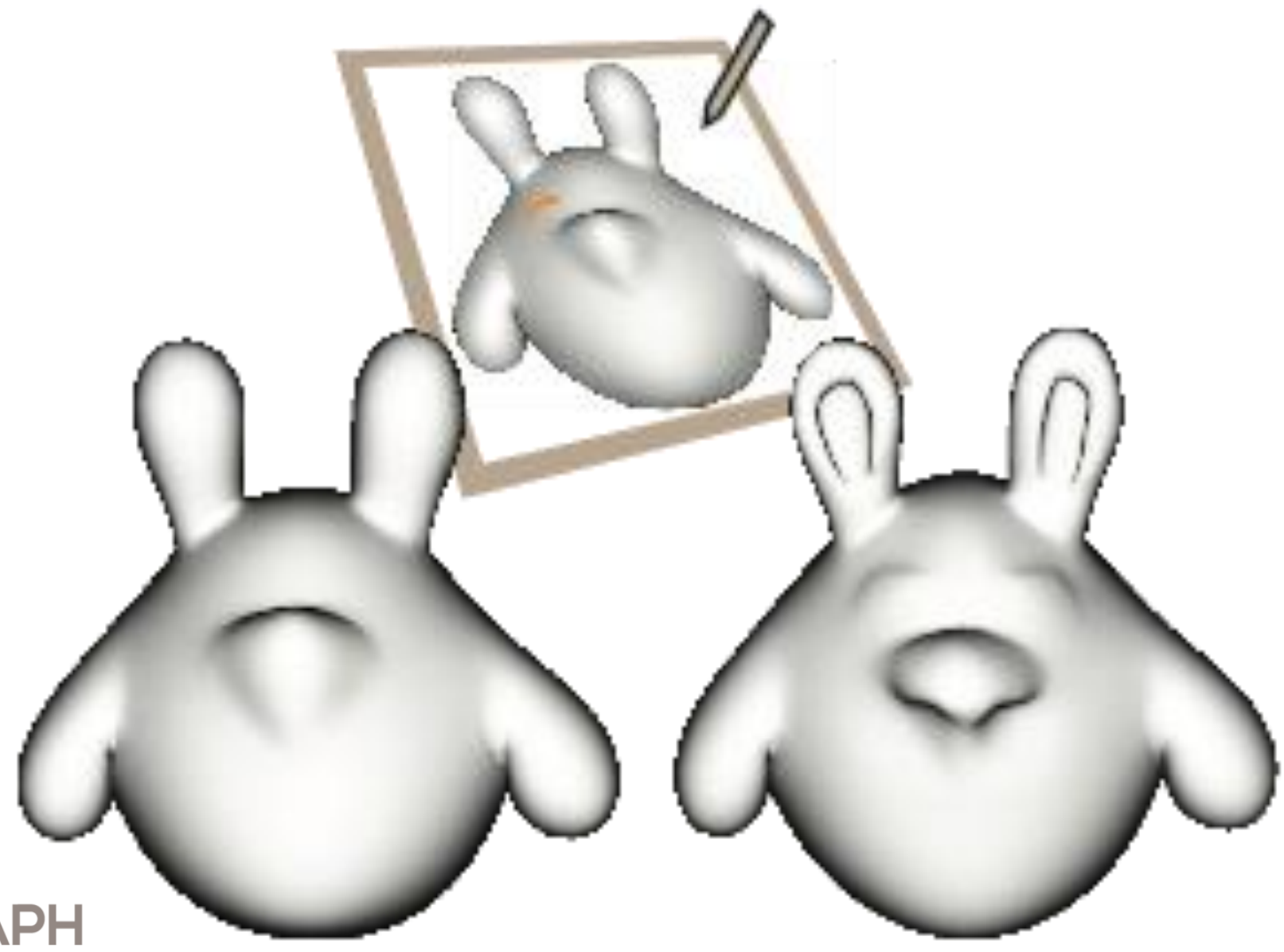




\section{Approach}

Obtain a new 3D model by shading over an existing one.

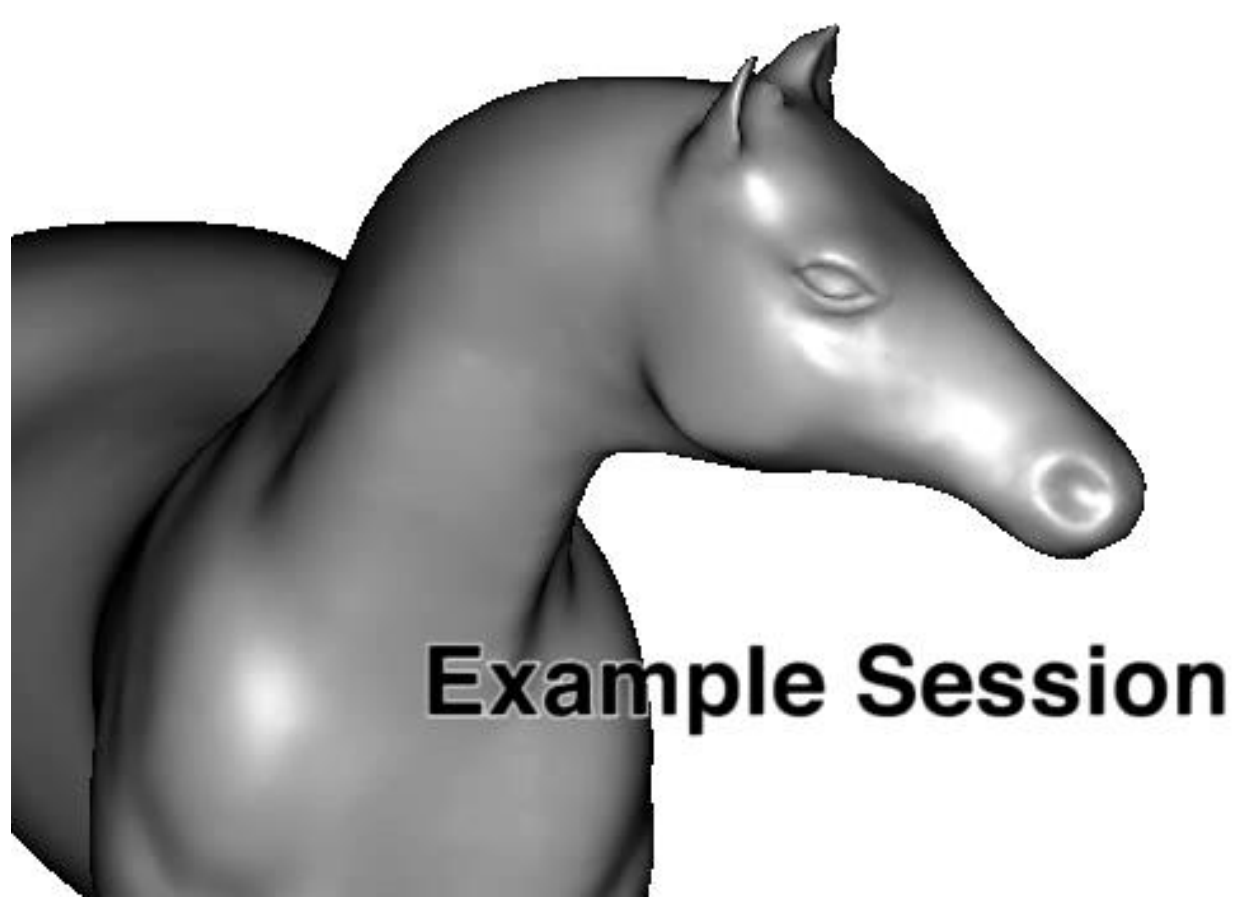




\section{Shape-from-Shading}

Given a shaded image of an object, can we recover its shape?

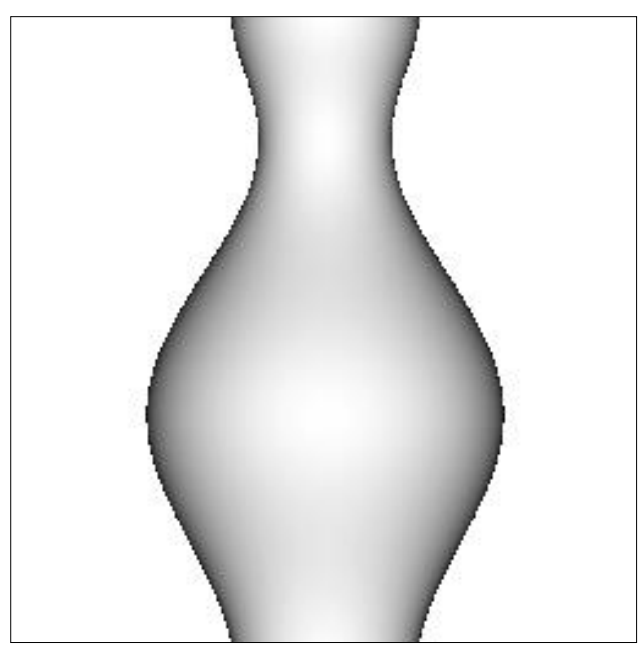

Shaded Image

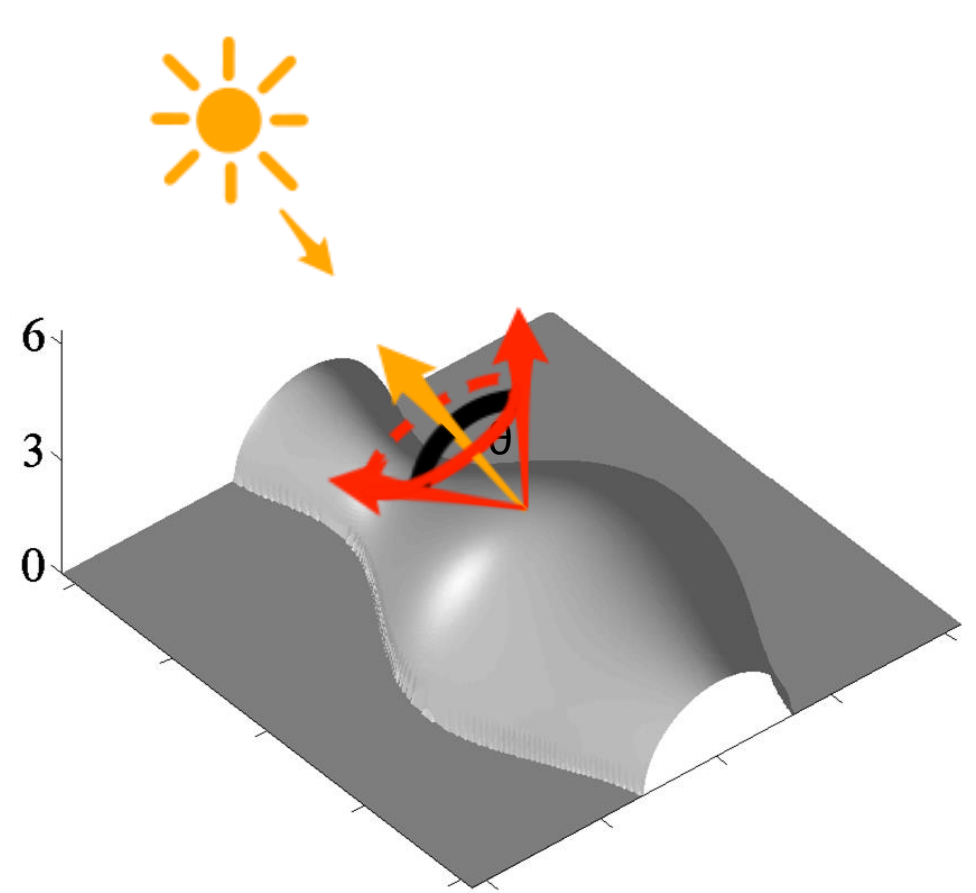

Shape (Height Field) 


\section{User Interface}

Q19. SIGGRAPH

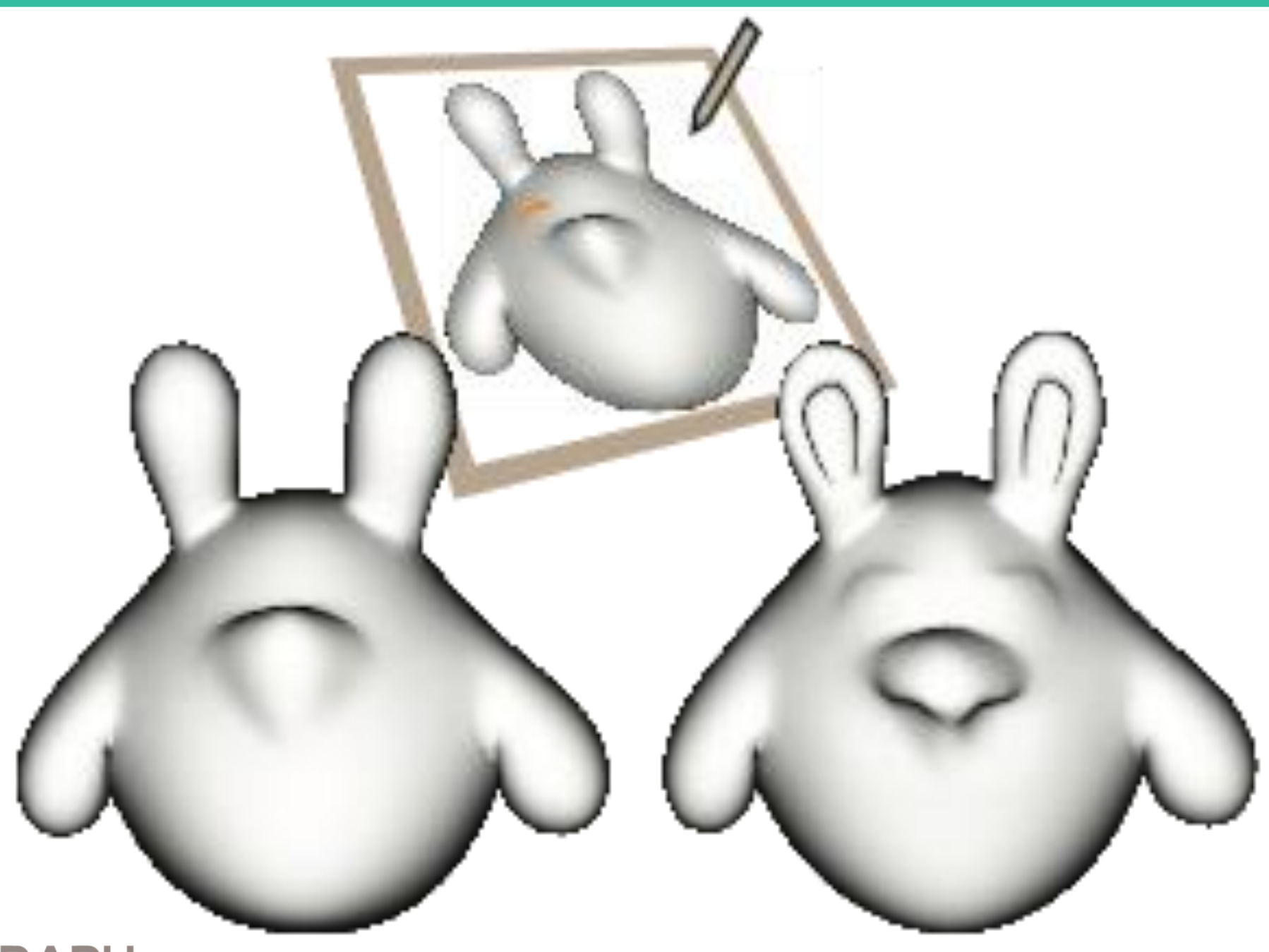

- ASIA 2916 


\section{Shading Strokes}

Thin Strokes 


\section{Silhouette Stroke}

Silhouette Strokes 


\section{Results}
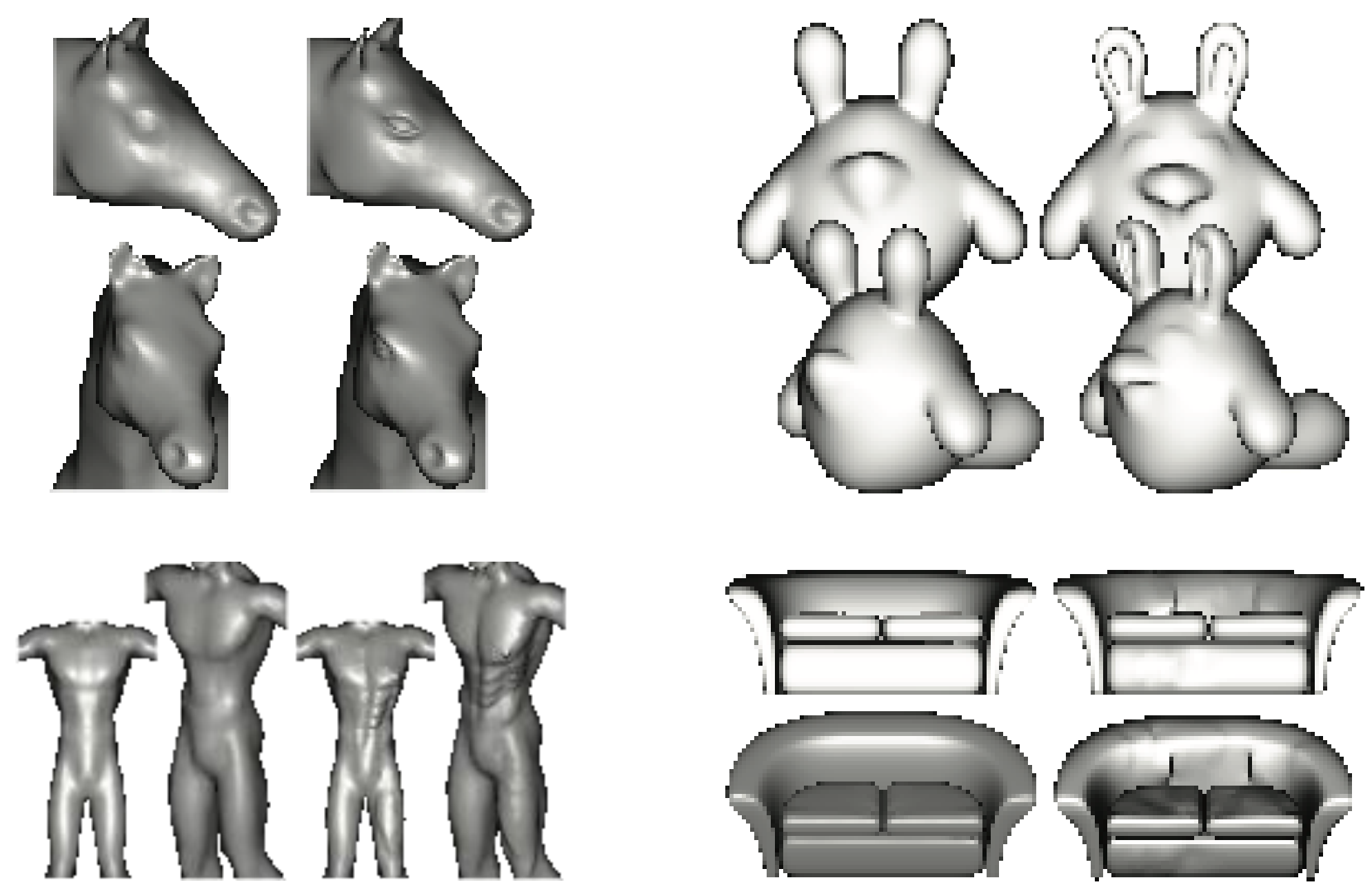

Q\%, SIGGRAPH

- ASIA 2916 


\section{Why is this hard?}

QQ, SIGGRAPH

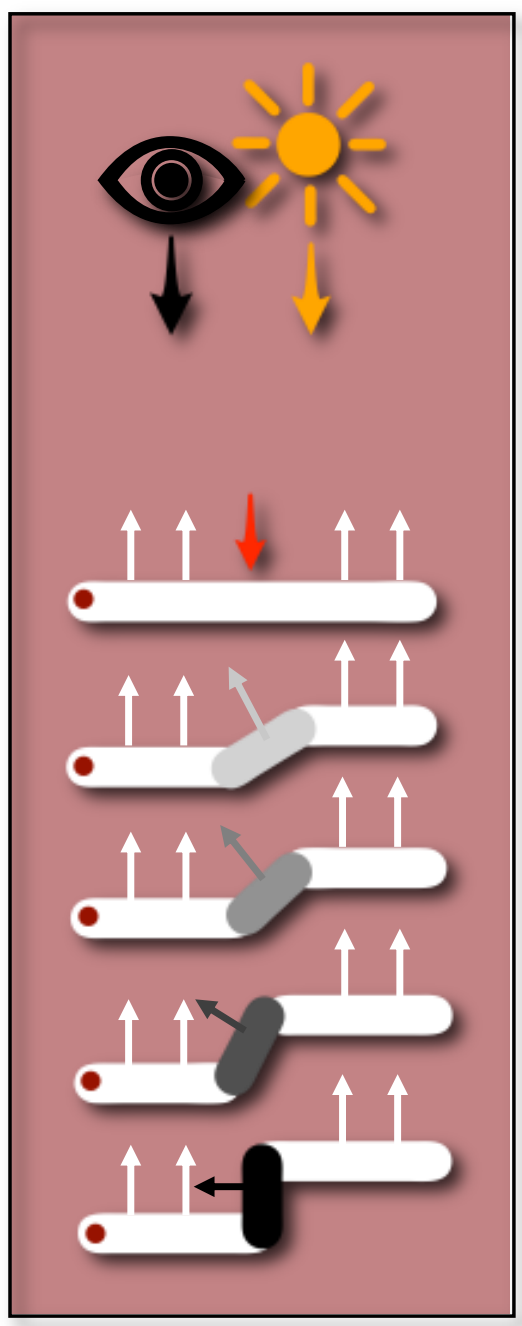

ASIA 2016 


\section{Why is this hard?}

The change is global

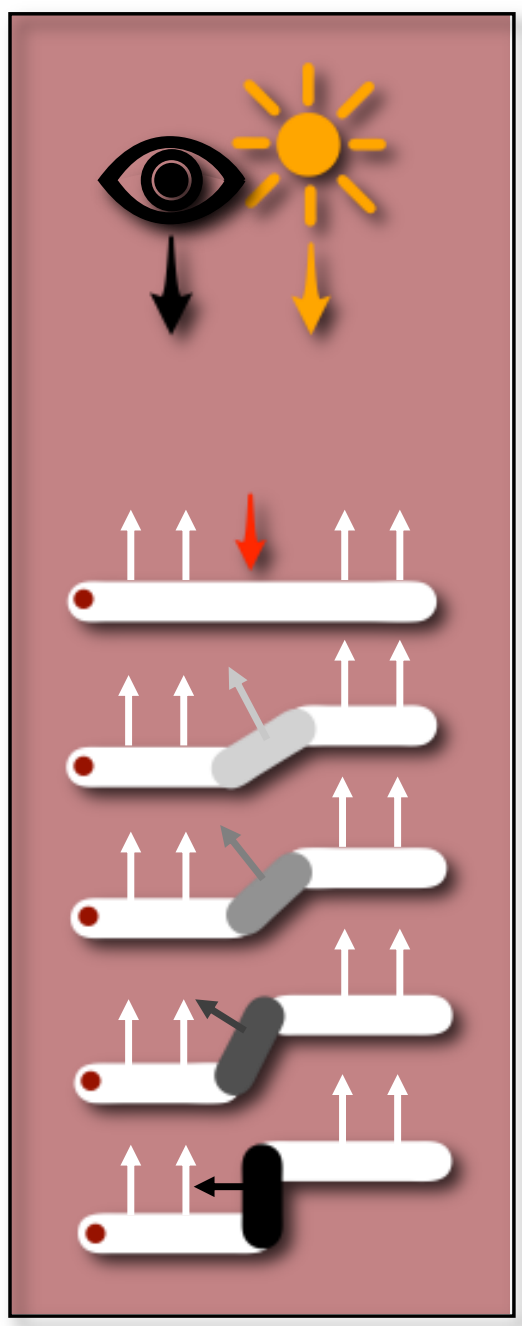




\section{Why is this hard?}

The change is global

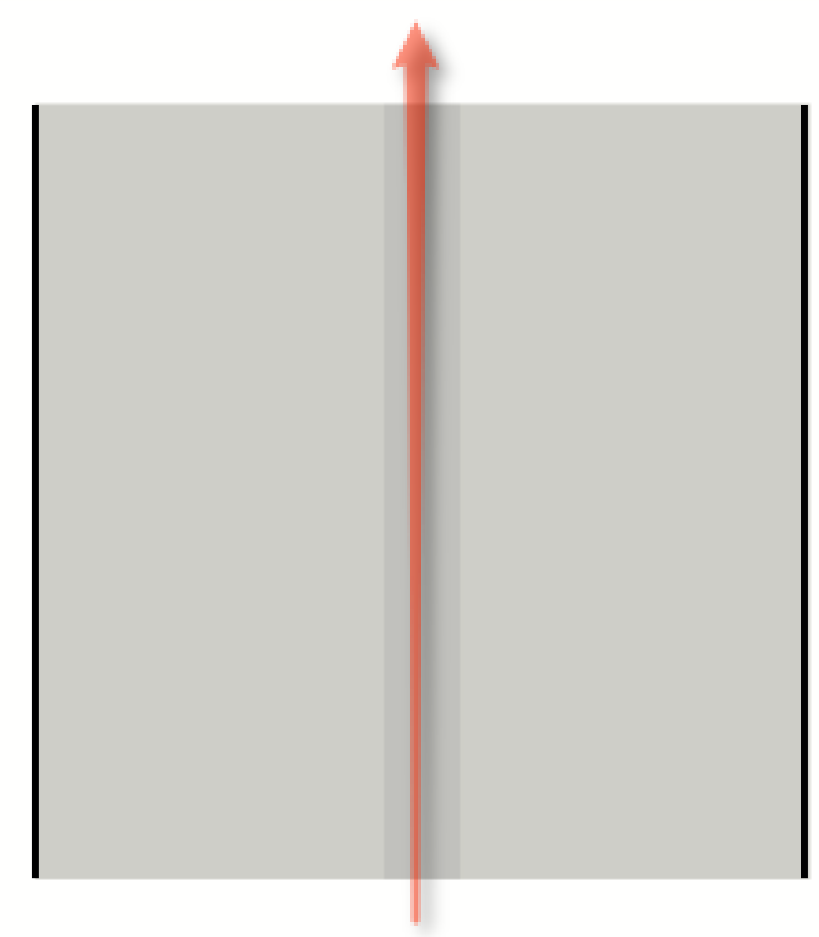

a.
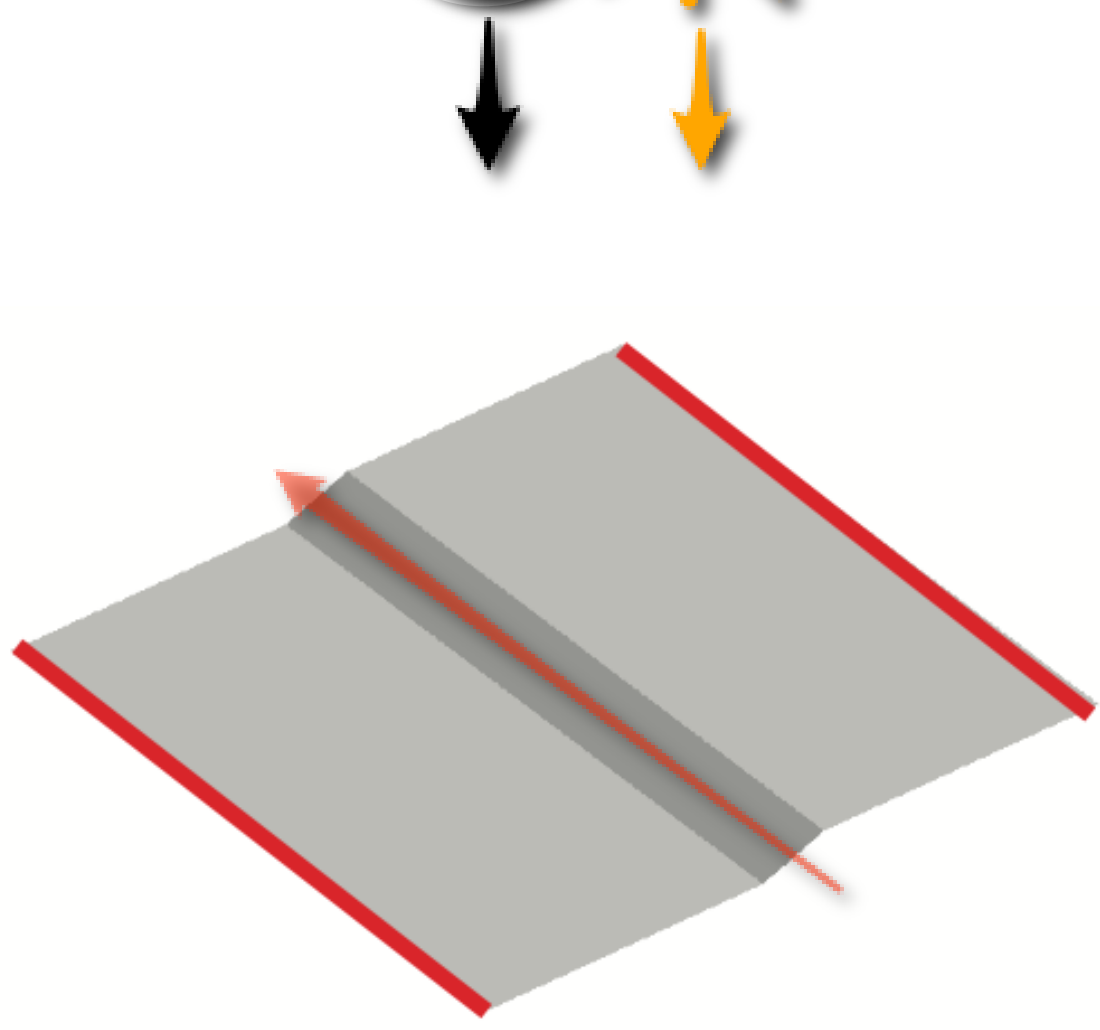

QQ, SIGGRAPH

ASIA 2016 


\section{Rotation about the stroke}

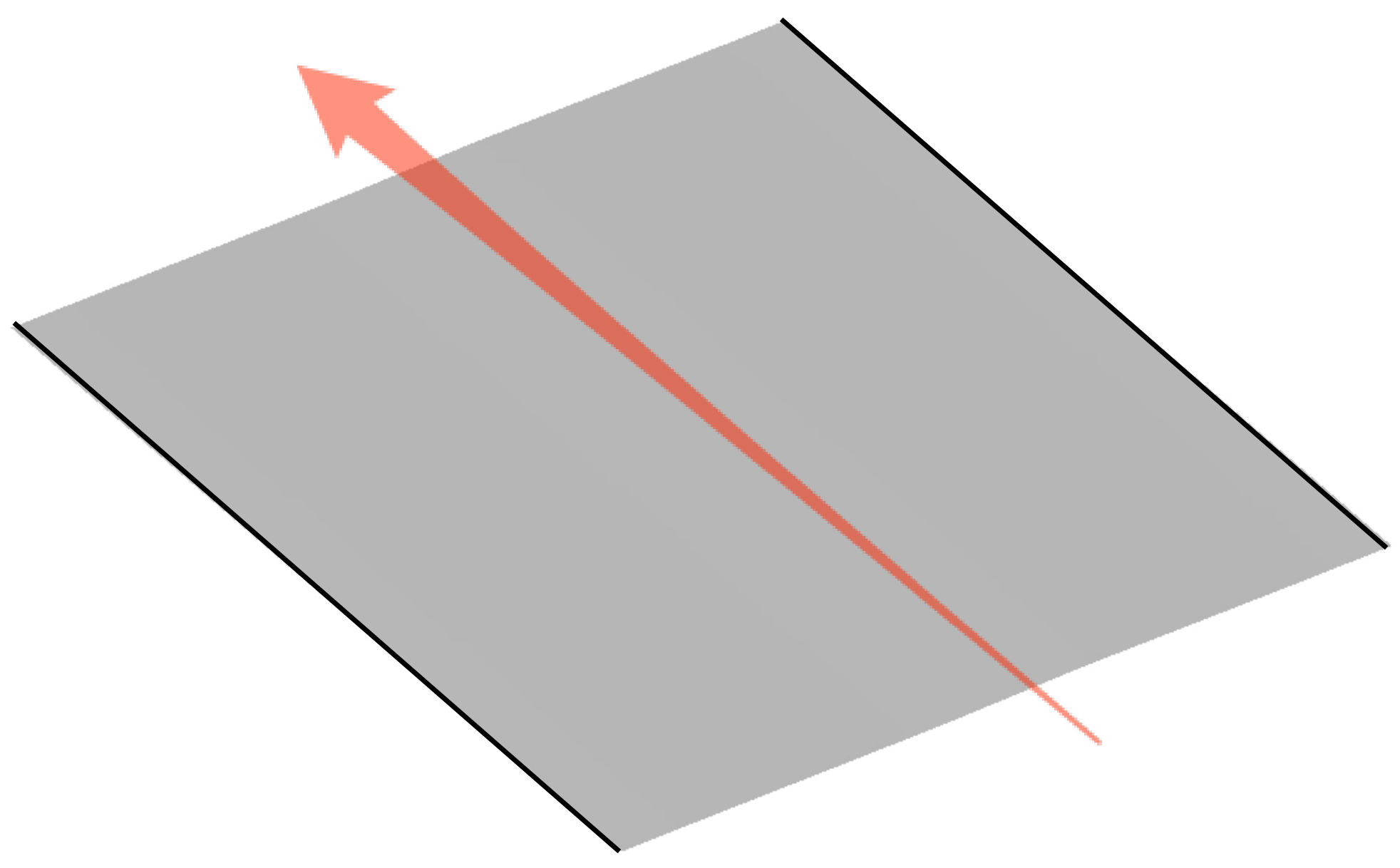

QQ. SIGGRAPH

- ASIA 2016 


\section{Approach}

Centerline of stroke: rotate surface about the stroke

- stable, predictable

Elsewhere: Laplacian Editing Energy [Sorkine et al. 2004]

- preserves appearance $\&$ shape

Variable vertex weights for our brush parameters

- controllable

Linear Constraints + Quadratic Energy = sparse linear system of equations

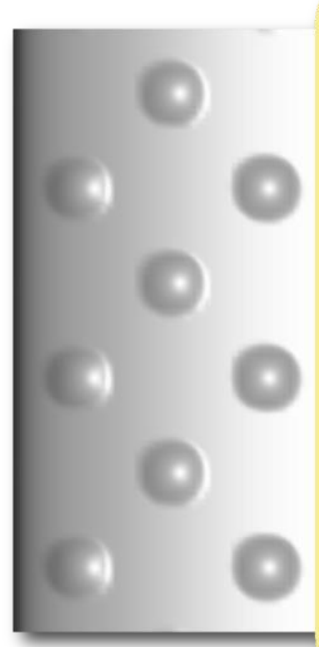




\section{Flip Ambiguity}

top

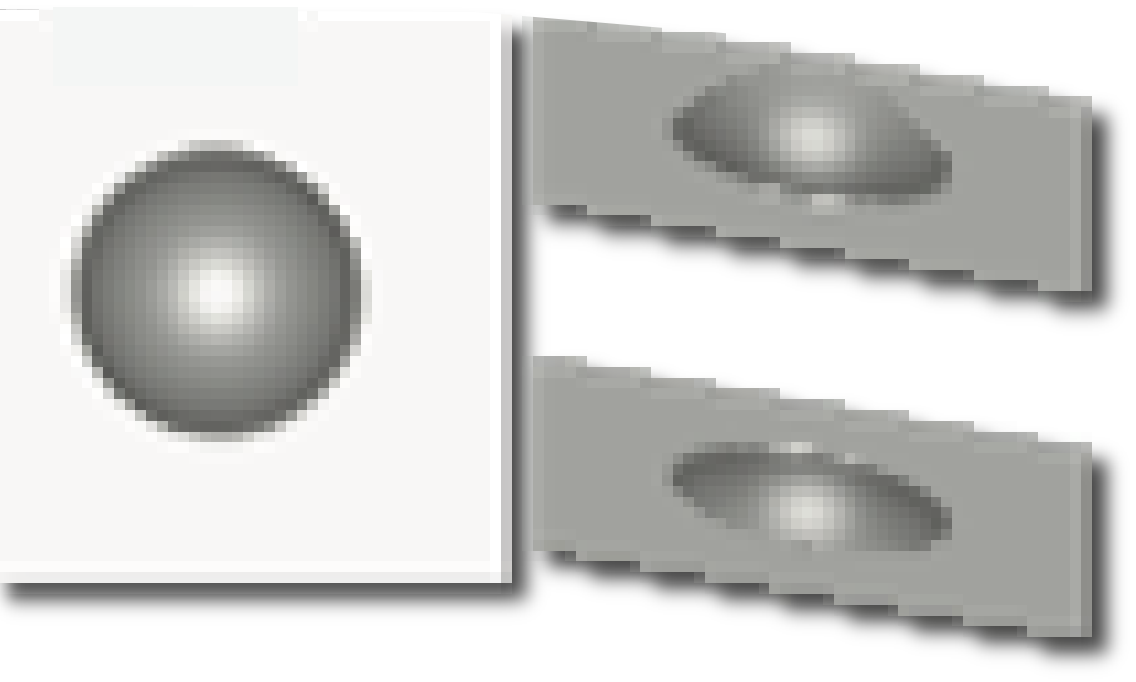

Concave/Convex ambiguity top

side

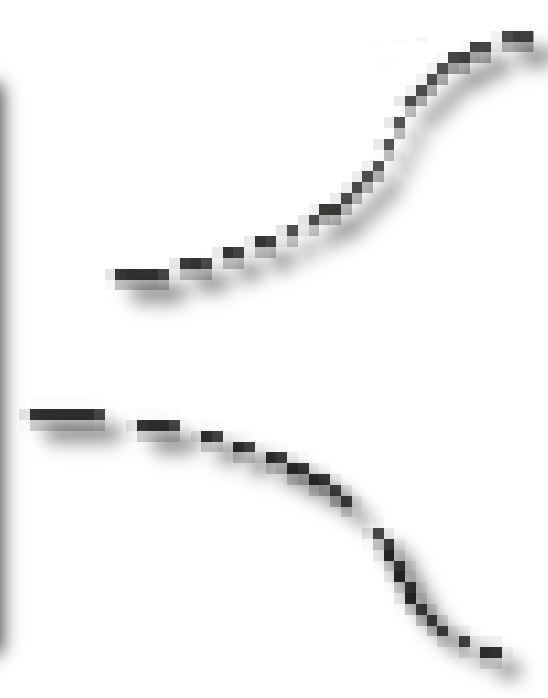

Slope

ambiguity 


\section{Summary}

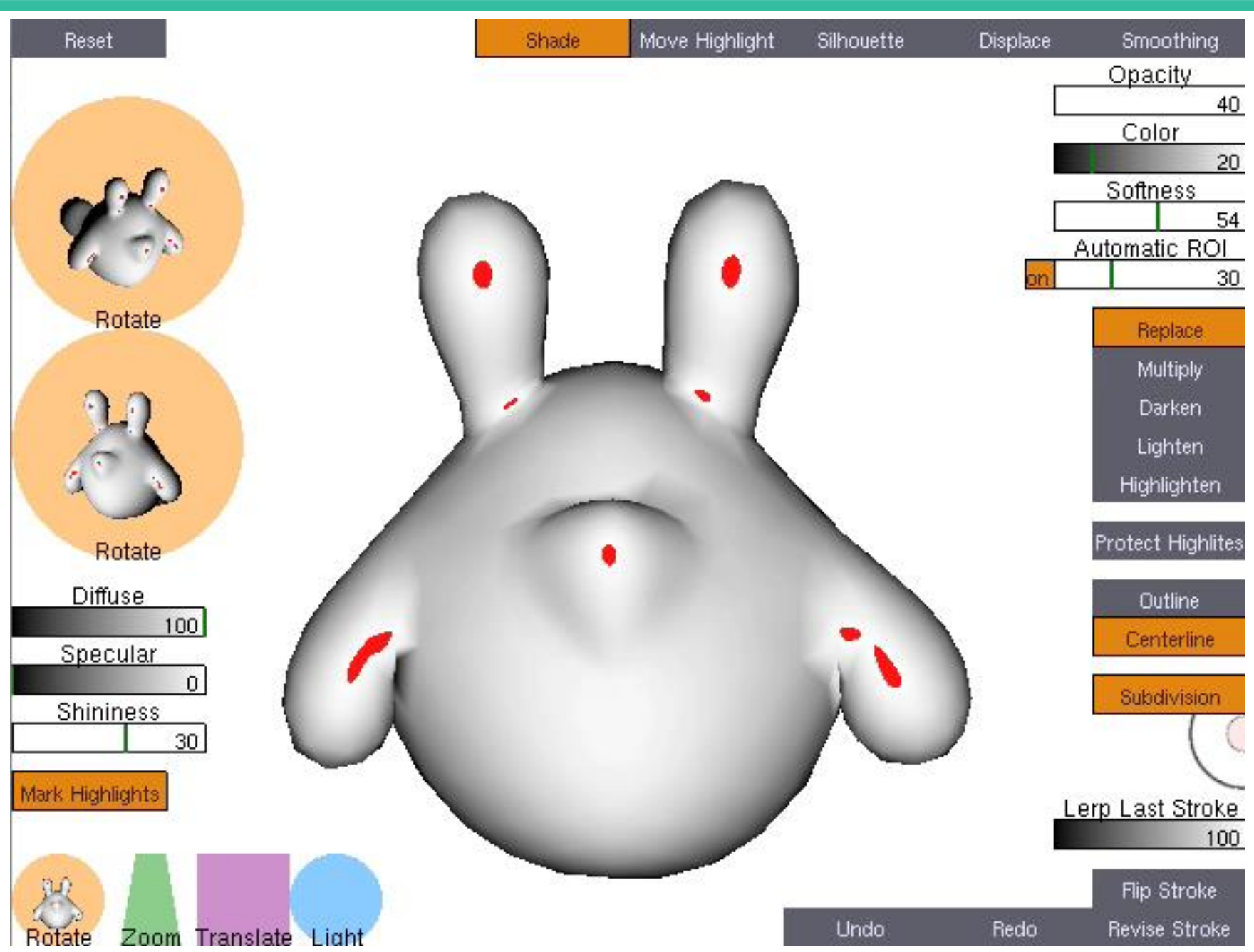

Q19, SIGGRAPH

ASIA 2016 


\section{Limitations}

Shading requires expertise.

Speed: modifications aren't local.

Highlight control is limited. 


\section{Takeaways}

- Familiar curves (silhouettes and shading) are natural candidates for editing operations.

- Sketching is a natural way to input curves.

- It's "easier" to deform than create geometry. 